\title{
Standalone BISON Fuel Performance Results for Watts Bar Unit 1, Cycles 1-3
}

Shane Stimpson

Kevin Clarno

Jeffrey Powers

Oak Ridge National Laboratory

Roger Pawlowski

Sandia National Laboratory

March 7, 2016
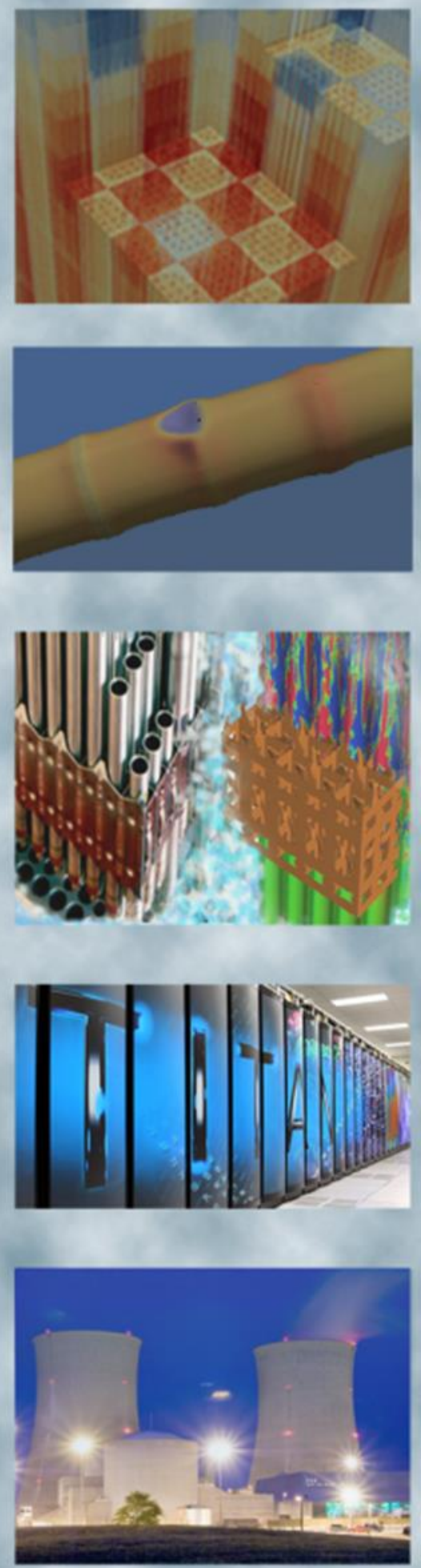

REVISION LOG

\begin{tabular}{|c|c|c|l|}
\hline Revision & Date & Affected Pages & \multicolumn{1}{c|}{ Revision Description } \\
\hline 0 & $12 / 18 / 2015$ & All & Original Report \\
\hline 1 & $03 / 07 / 2016$ & All & Report cleared for unlimited distribution \\
\hline & & & \\
\hline & & & \\
\hline
\end{tabular}

\section{Document pages that are:}

Export Controlled __ NO

IP/Proprietary/NDA Controlled __ NO

Sensitive Controlled __ NO

Approved for Public Release ___ YES 


\section{EXECUTIVE SUMMARY}

The Consortium for Advanced Simulation of Light Water Reactors (CASL) is moving forward with more complex multiphysics simulations and increased focus on incorporating fuel performance analysis methods. The coupled neutronics/thermal-hydraulics capabilities within the Virtual Environment for Reactor Applications Core Simulator (VERA-CS) have become relatively stable, and major advances have been made in analysis efforts, including the simulation of twelve cycles of Watts Bar Nuclear Unit 1 (WBN1) operation. While this is a major achievement, the VERA-CS approaches for treating fuel pin heat transfer have well-known limitations that could be eliminated through better integration with the BISON fuel performance code.

Several approaches are being implemented to consider fuel performance, including a more direct multiway coupling with Tiamat, as well as a more loosely coupled one-way approach with standalone BISON cases. Fuel performance typically undergoes an independent analysis using a standalone fuel performance code with manually specified input defined from an independent core simulator solution or set of assumptions.

This milestone covers efforts to facilitate the use of VERA for core simulation and fuel performance to operate in this standalone mode of execution by using power distribution and moderator temperature data from VERA-CS. This approach can be used for several CASL fuel performance applications:

1) as a screening tool for the pellet-clad interaction (PCI) challenge problem to identify rods that require further analysis,

2) to analyze reactivity insertion accidents (RIAs) and departures from nucleate boiling (DNBs) which require time-dependent full core results to establish the boundary conditions for the high-fidelity BISON simulations, and

3) to improve fuel temperature models in VERA-CS by providing higher fidelity comparisons of core-wise temperature distributions.

4) as a high fidelity fuel performance methodology (using explicit rather than bounding core operating conditions) to evaluate and screen fuel and core design concepts for a comprehensive set of design criteria including pin powers, PCI, pin burnups, corrosion, oxidation, margin to fuel melt, and rod internal pressure.

This report summarizes the improvements made since the initial milestone to execute BISON from VERA-CS output. Many of these improvements were prompted through tighter collaboration with the BISON development team at Idaho National Laboratory (INL). A brief description of WBN1 and some of the VERA-CS data used to simulate it are presented. Data from a small mesh sensitivity study are shown, which helps justify the mesh parameters used in this work. The multi-cycle results are presented, followed by the results for the first three cycles of WBN1 operation, particularly the parameters of interest to PCI screening (fuel-clad gap closure, maximum centerline fuel temperature, maximum/minimum clad hoop stress, and cumulative damage index). Once the mechanics of this capability are functioning, future work will target cycles with known or suspected PCI failures to determine how well they can be estimated. 


\section{CONTENTS}

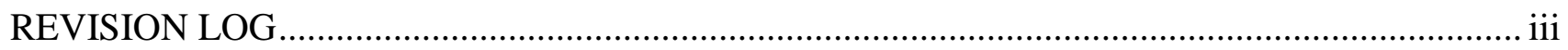

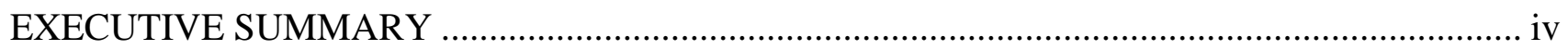

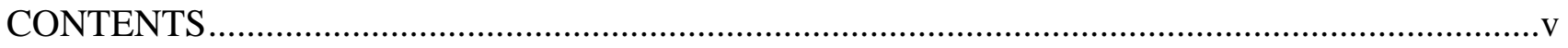

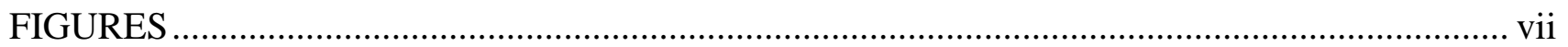

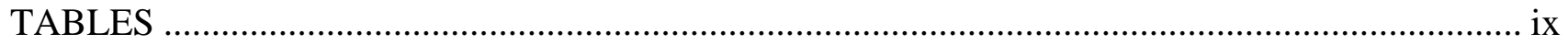

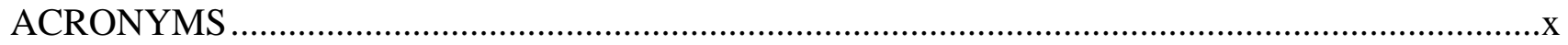

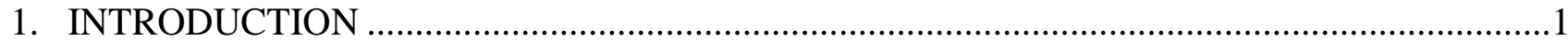

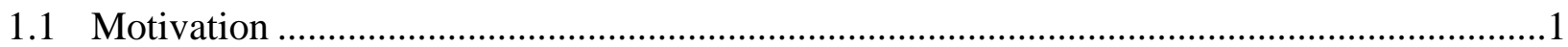

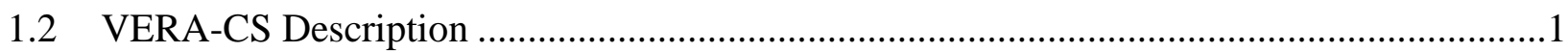

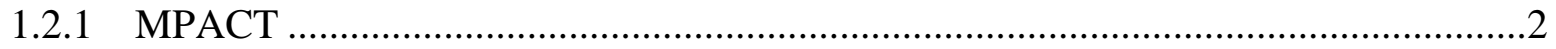

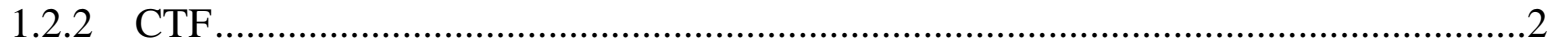

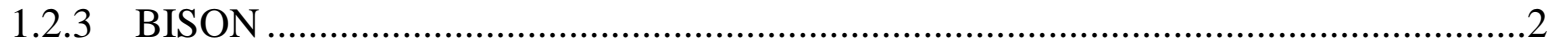

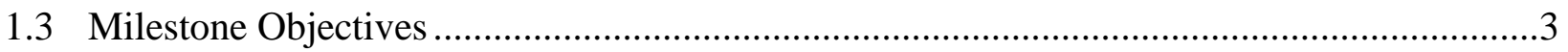

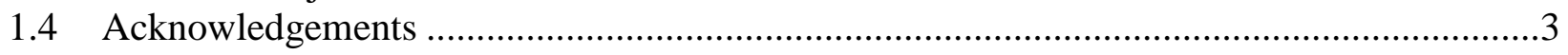

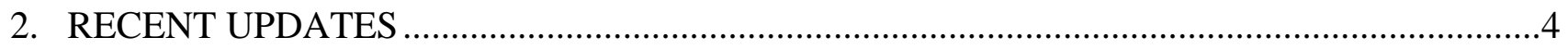

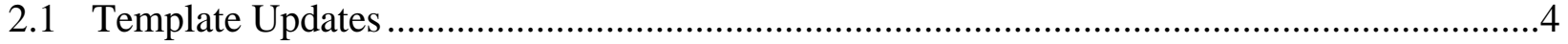

2.2 XML2MOOSE Preprocessor Updates ....................................................................

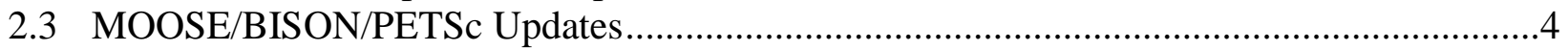

2.4 Unit Testing Additions ............................................................................................

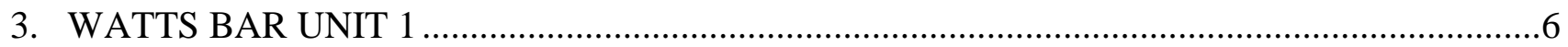

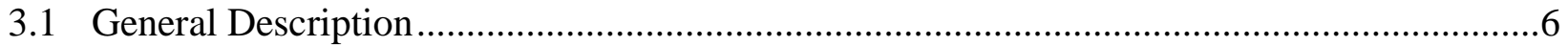

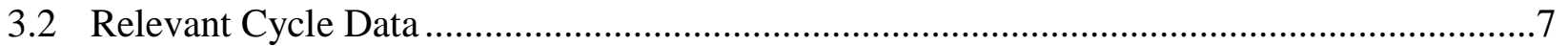

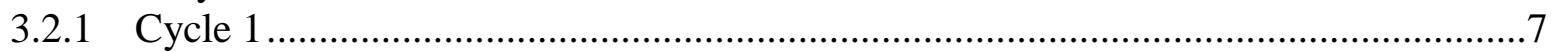

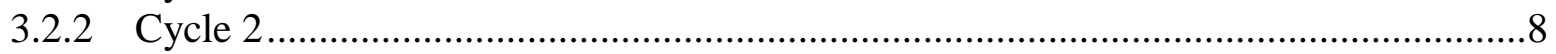

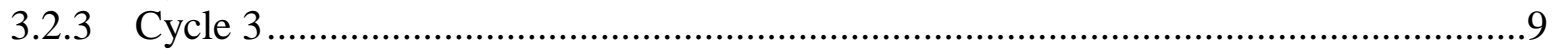

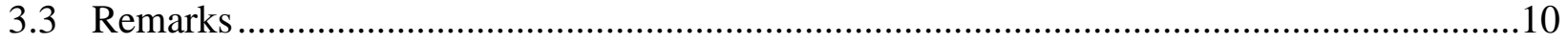

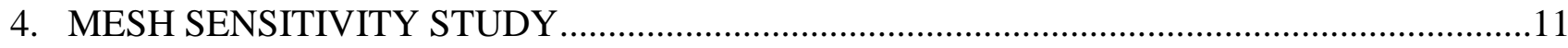

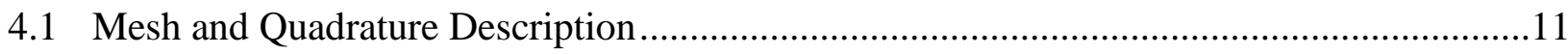

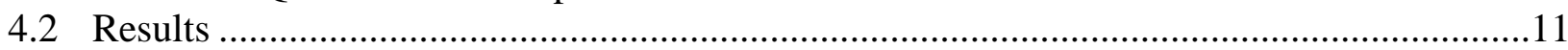

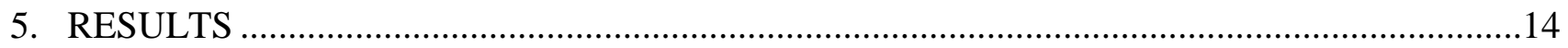

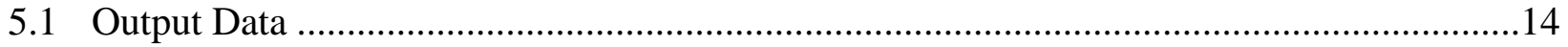

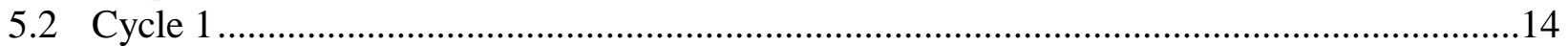

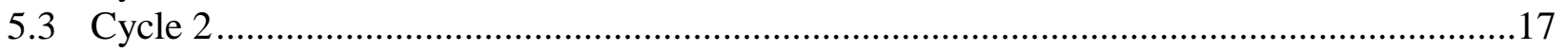

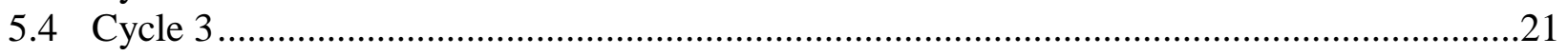

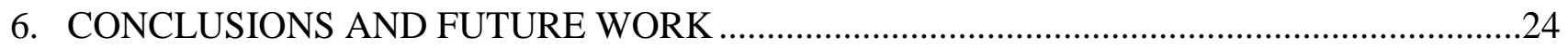

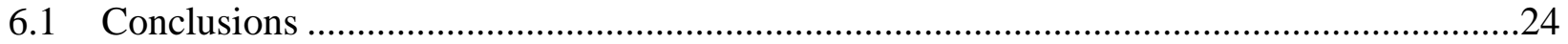

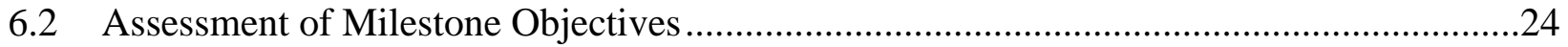


6.3 Running on Leadership Class Clusters .......................................................................25

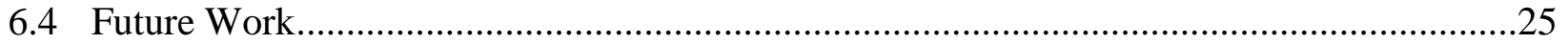

6.4.1 Accounting for More Fuel Rod Types...........................................................25

6.4.2 Biased Meshing .............................................................................................25

6.4.3 Assess Effect of ZIRLO vs. Zirc-4 .................................................................25

6.4.4 Oxide Layer Growth ................................................................................25

6.4.5 Processing More Detailed Output via EXODUS .............................................25

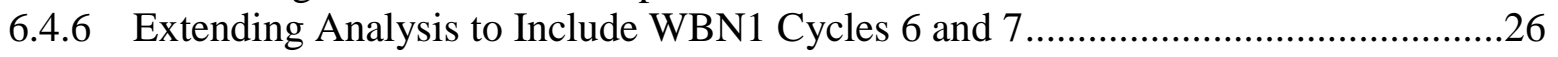

6.4.7 Assessing Power Ramp Procedures..............................................................26

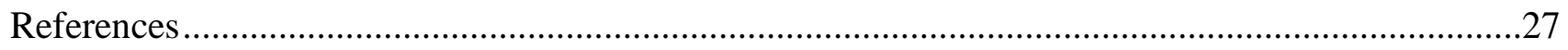

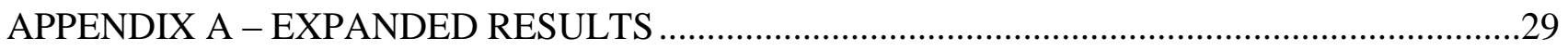

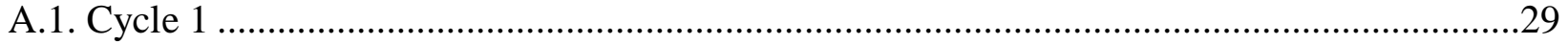

A.1.1. Maximum Centerline Fuel Temperature .......................................................29

A.1.2. Minimum Gap Thickness ............................................................................... 41

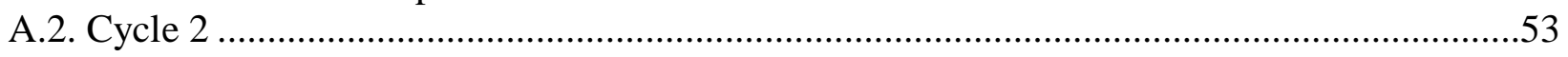

A.2.1. Maximum Centerline Fuel Temperature ............................................................53

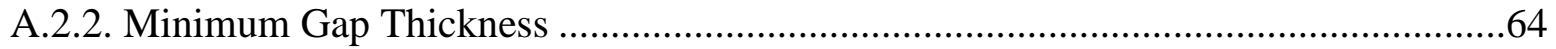

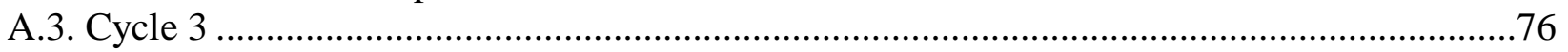

A.3.1. Maximum Centerline Fuel Temperature .....................................................76

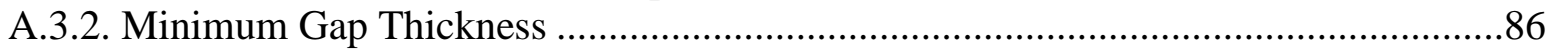

APPENDIX B - UPDATED BISON INPUT TEMPLATE .....................................................96 


\section{FIGURES}

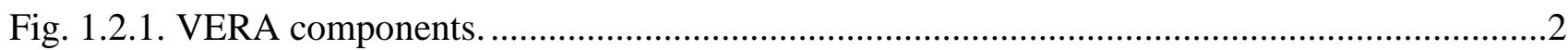

Fig. 3.1.1. Watts Bar Unit 1 - Cycle 1 Core diagram [1] ........................................................6

Fig. 3.1.2. Watts Bar Unit 1 - Cycle 1 core layout and rod bank configuration. ..............................

Fig. 3.2.1. Watts Bar Unit 1 - Cycle 1 power history ................................................................8

Fig. 3.2.2. Watts Bar Unit 1 - Cycle 2 power history ..............................................................

Fig. 3.2.3. Watts Bar Unit 1 - Cycle 2 core layout.................................................................

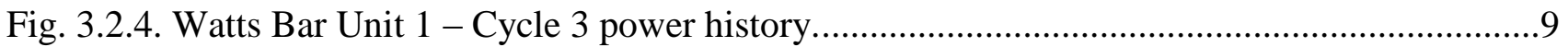

Fig. 3.2.5. Watts Bar Unit 1 - Cycle 3 core layout.............................................................. 10

Fig. 4.2.1. Mesh sensitivity study - maximum centerline fuel temperature $(\mathrm{K})$...........................11

Fig. 4.2.2. Mesh sensitivity study - average fuel temperature $(\mathrm{K})$.............................................12

Fig. 4.2.3. Mesh sensitivity study - minimum gap thickness $(\mu \mathrm{m})$.............................................. 12

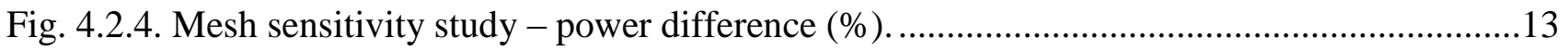

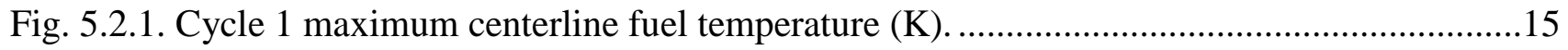

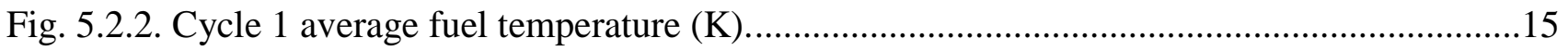

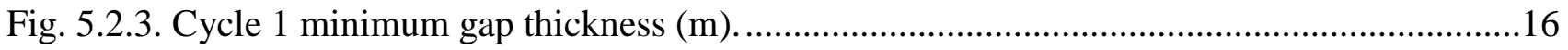

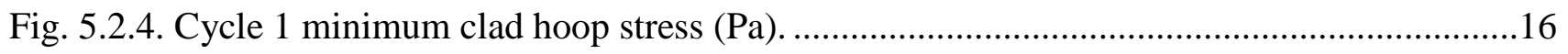

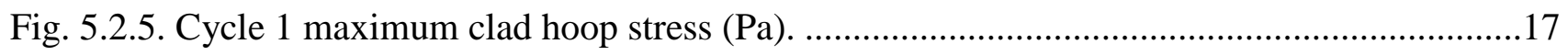

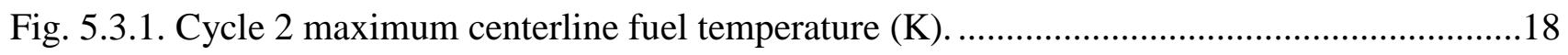

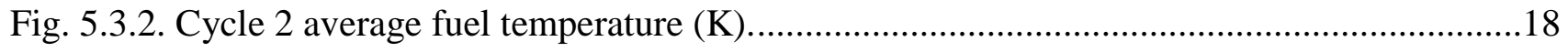

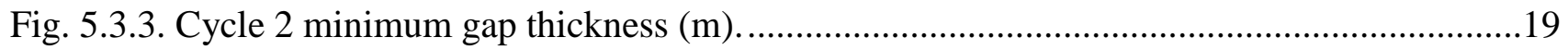

Fig. 5.3.4. Cycle 2 minimum clad hoop stress $(\mathrm{Pa})$.............................................................. 19

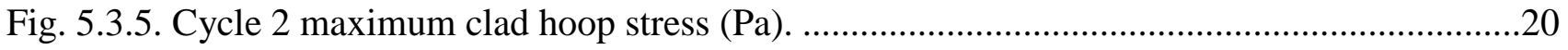

Fig. 5.3.6. Cycle 2 cumulative damage index...................................................................20

Fig. 5.4.1. Cycle 3 maximum centerline fuel temperature $(\mathrm{K})$...............................................21

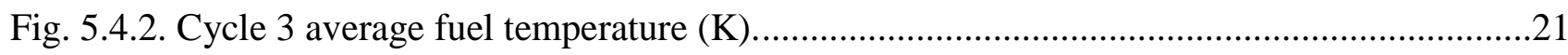




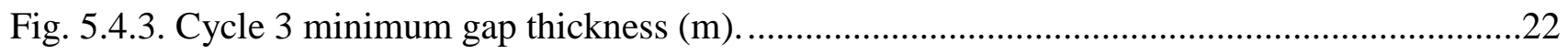

Fig. 5.4.4. Cycle 3 minimum clad hoop stress $(\mathrm{Pa})$......................................................................22

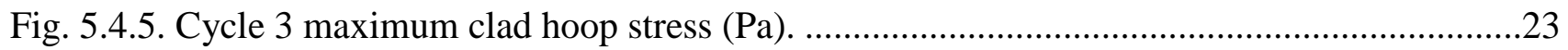

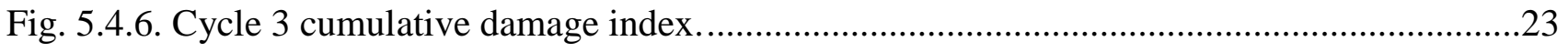




\section{TABLES}

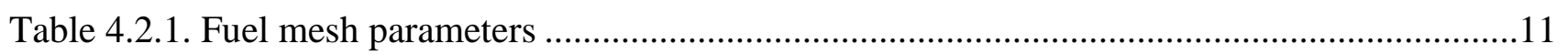

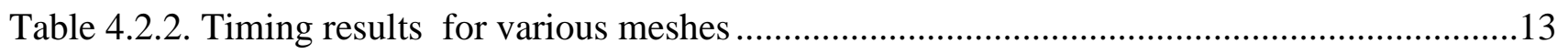




\section{ACRONYMS}

\begin{tabular}{|c|c|}
\hline ANS & American Nuclear Society \\
\hline $\mathrm{BOC}$ & beginning of cycle \\
\hline CASL & Consortium for Advanced Simulation of Light Water Reactors \\
\hline CIPS & crud-induced power shift \\
\hline CMFD & coarse mesh finite difference \\
\hline COBRA & Coolant Boiling in Rod Arrays \\
\hline CS & core simulator \\
\hline CSV & comma separated value \\
\hline CTF & COBRA two-phase flow \\
\hline DNB & departure from nucleate boiling \\
\hline DOE & US Department of Energy \\
\hline EFPD & effective full power day \\
\hline GWd/MT & gigawatt day per metric ton \\
\hline $\mathrm{HDF}$ & hierarchical data format \\
\hline IFBA & integral fuel burnable absorber \\
\hline INF & infrastructure \\
\hline INL & Idaho National Laboratory \\
\hline JFNK & Jacobian Free Newton-Krylov \\
\hline LWR & light water reactor \\
\hline $\mathrm{MOC}$ & Method of Characteristics \\
\hline MOOSE & Multiphysics Object Oriented Simulation Environment \\
\hline MW & megawatt \\
\hline MWth & megawatt thermal \\
\hline $\mathrm{NE}$ & Office of Nuclear Energy \\
\hline NEM & nodal expansion method \\
\hline ORNL & Oak Ridge National Laboratory \\
\hline PCI & pellet-clad interaction \\
\hline PETSc & Portable, Extensible Toolkit for Scientific Computation \\
\hline PWR & pressurized water reactor \\
\hline RIA & reactivity-insertion accident \\
\hline $\mathrm{SP}_{3}$ & simplified $\mathrm{P}_{3}$ \\
\hline $\mathrm{TH}$ & thermal hydraulics \\
\hline TPL & third party library \\
\hline TVA & Tennessee Valley Authority \\
\hline UM & University of Michigan \\
\hline VERA & Virtual Environment for Reactor Applications \\
\hline WABA & wet annular burnable absorber \\
\hline WBN1 & Watts Bar Nuclear Unit 1 \\
\hline
\end{tabular}




\section{INTRODUCTION}

\subsection{Motivation}

The Consortium for Advanced Simulation of Light Water Reactors (CASL) is moving forward with more complex multiphysics simulations, and there is increased focus on incorporating fuel performance analysis methods into the Virtual Environment for Reactor Applications Core Simulator (VERA-CS). The coupled neutronics/thermal-hydraulics (TH) capabilities within VERA-CS are relatively stable, and major advances have been made in analysis efforts, including the simulations of twelve cycles of Watts Bar Nuclear Unit 1 (WBN1) operation [1]. Several approaches are being implemented to consider fuel performance, including a more direct multiway coupling with Tiamat $[2,3,4]$, as well as a more loosely coupled one-way approach with standalone BISON cases.

This milestone focuses on generating and executing BISON fuel performance cases using output power and temperature distributions produced by coupled neutronics/TH simulations with VERACS. While work is underway to develop Tiamat, the primary motivation for this approach is to provide quicker results without the need to rerun previous calculations. For example, output from the twelve cycles of WBN1 simulated with MPACT and CTF within VERA-CS [5] could be used as a basis for independent BISON cases to assess parameters of pellet-clad interaction or accuracy of global fuel temperature distributions. Additionally, the results can provide insights for evaluating a number of fuel and core design concepts such as corrosion, oxidation, margin to fuel melt, and rod internal pressure. However, it should be noted that the VERA-CS simulations producing the temperature and power distribution yield a different fuel temperature because it is using a simpler fuel temperature table produced by BISON.

\subsection{VERA-CS Description}

The VERA simulation environment being developed by CASL is comprised of codes collectively used for nuclear reactor modeling and simulation. This work uses the MPACT neutron transport solver, the CTF TH solver, and the BISON fuel performance code, all briefly described below. Figure 1.2.1 shows the components of VERA. At this writing, the components that make up the core simulator (VERA-CS) are the neutronics, TH, and fuel performance packages. 


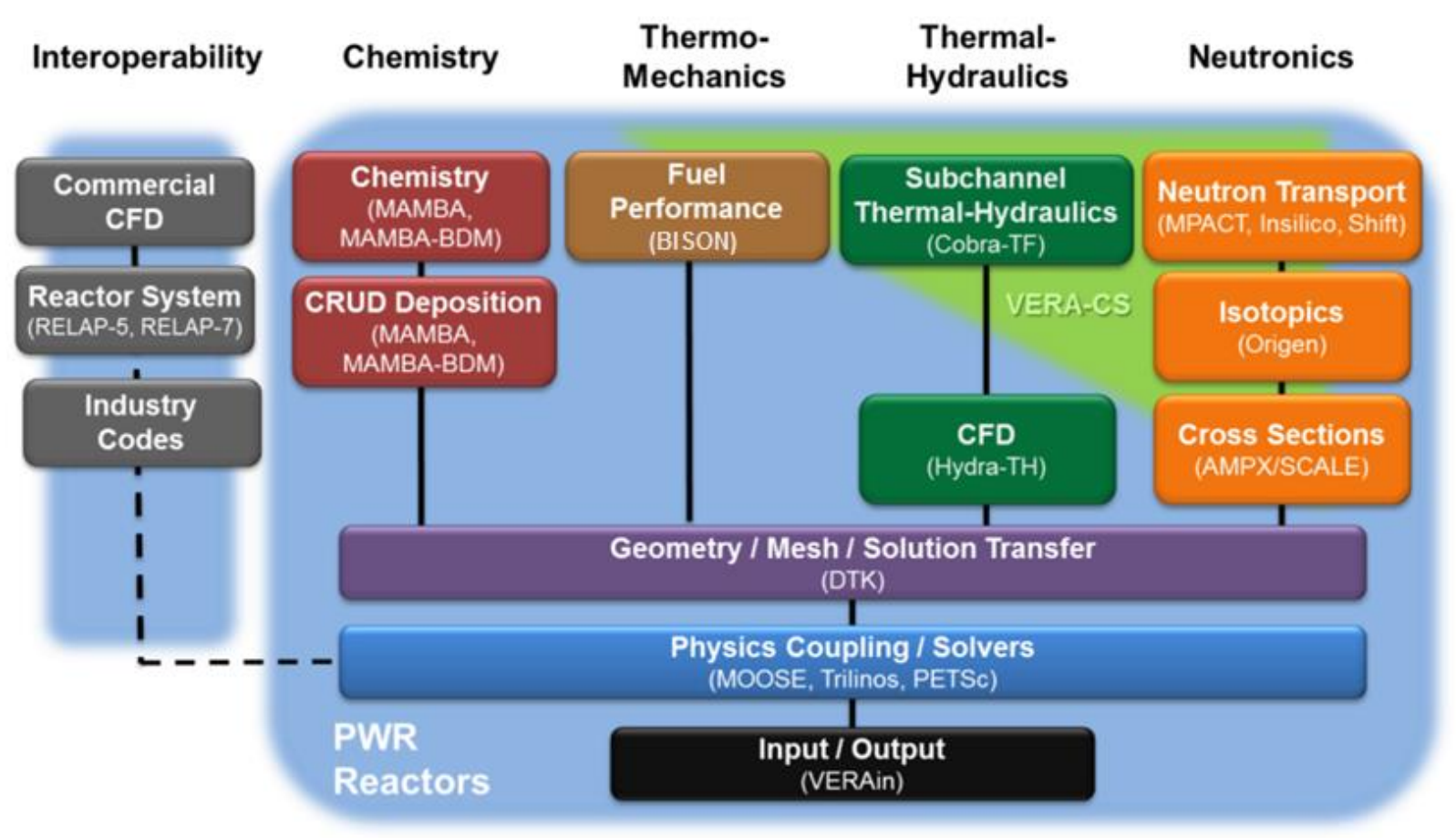

Fig. 1.2.1. VERA components.

\subsubsection{MPACT}

The MPACT neutron transport solver, being developed collaboratively by Oak Ridge National Laboratory (ORNL) and the University of Michigan (UM), provides pin-resolved flux and power distributions [6]. To solve three-dimensional (3D) problems, it employs the 2D/1D method, which decomposes the problem into a 1D axial stack of 2D radial planes [7]. Typically, 2D Method of Characteristics (2D MOC) is used to solve each radial plane, and 1D nodal methods are used to solve axially along each rod. While there are a variety of axial solvers available, the nodal expansion method (NEM)-simplified $\mathrm{P}_{3}\left(\mathrm{SP}_{3}\right)$ solver is the default, which wraps a one-node NEM kernel [8]. These 2D and 1D solvers are coupled together through transverse leakage terms to ensure neutron conservation, and they are accelerated using 3D coarse mesh finite difference (CMFD).

\subsubsection{CTF}

CTF is a subchannel TH code being developed by ORNL and Pennsylvania State University (PSU) specifically for light water reactor (LWR) analysis [10]. It simulates two-phase flow with a threefield representation - liquid, droplet, and vapor-assuming that the liquid and droplet fields are in dynamic equilibrium, leaving two energy conservation equations.

CTF provides significantly higher resolution than internal thermal hydraulics solver (Simplified TH) in MPACT, but it is currently more limited in parallelization. Ongoing work is focused on increasing parallelization from the assembly level to the subchannel level, significantly alleviating runtime concerns for cases presented in this work.

\subsubsection{BISON}

The BISON fuel performance code is being developed by Idaho National Laboratory (INL) to provide single-rod fuel performance modeling capability so that users can assess best-estimate values of design and safety criteria and the impact of plant operation and fuel rod design on thermomechanical behavior such as pellet-cladding interaction (PCI) failures in pressurized water reactors (PWRs) $[11,12]$. PCI is controlled by the complex relationship between the mechanical, thermal, and 
chemical behaviors of a fuel rod during operation. Consequently, modeling PCI requires an integral fuel performance code to simulate the fundamental processes of these behaviors. BISON is built on INL's Multiphysics Object Oriented Simulation Environment (MOOSE) package [13,14], which use the finite element method for geometric representation and a Jacobian Free Newton-Krylov (JFNK) scheme to solve systems of partial differential equations [13]. For this work, BISON uses a 2D azimuthally symmetric (R-Z), smeared-pellet thermomechanical fuel pin model with output data from VERA-CS to generate the time-dependent power shape/history and moderator temperature inputs needed for BISON.

\subsection{Milestone Objectives}

There are a number of primary objectives in this milestone, several of which build upon work from the initial milestone [15]:

1) extend XML2MOOSE to accommodate fuel shuffling in quarter symmetry cases as quarter symmetry assumptions were used in both this work and the VERA-CS simulations;

2) develop a post-processor to collect the results from the individual BISON cases and consolidate them onto the VERAOut hierarchical data format (HDF) 5 file, particularly for use in VERAView;

3) consider the establishment of a driver program that will simplify usage by preprocessing, running, and gathering output all in one overarching executable;

4) determine an appropriate model for simulating integral fuel burnable absorber (IFBA) rods in BISON;

5) run several cycles of WBN1 using the VERA-CS output data available from Reference 1; and

6) assess temperatures, stresses, and other important parameters of interest to PCI screening.

With two additional stretch goals:

1) compare results to FRAPCON results obtained by Ryan Bratton [16], a student at Pennsylvania State University working remotely from ORNL, who obtained these results as part of his thesis work; and

2) perform a mesh sensitivity study to assess appropriate refinement level.

\subsection{Acknowledgements}

The authors wish to acknowledge the BISON development team for their continued support, as well as the CASL Infrastructure (INF) team, particularly Mark Baird and Ross Bartlett, for updating PETSc and MOOSE very quickly.

Many thanks are also due to Ron Lee for his expedient modifications to VERAView to allow for the new data structures being introduced with this work.

This this work was supported by the Consortium for Advanced Simulation of Light Water Reactors (www.casl.gov), an energy innovation hub (http://www.energy.gov/hubs) for modeling and simulation of nuclear reactors under US Department of Energy (DOE) Contract No. DE-AC0500OR22725.

This research used the resources of the High Performance Computing Center at INL, which is supported by DOE's Office of Nuclear Energy (NE) under Contract No. DE-AC07-05ID14517. 


\section{RECENT UPDATES}

Since completion of the initial milestone to generate BISON cases from VERA-CS output [15], several important modifications have been made to the BISON template, XML2MOOSE preprocessor, and general software configuration.

\subsection{Template Updates}

Meetings with the BISON development team and continued review have prompted a number of modifications to the BISON template file.

1) To help resolve some of the convergence issues observed during cycle outages, the BISON team recommended changing the problem type to what is termed a ReferenceResidual type. This has been more robust, particularly when there is not much change occurring to the input variables, as is the case during a cycle outage. As will be seen in the results, this change has been fairly successful overall, but there are still a couple of cases that fail to converge.

2) To address concerns with lengthy runtime, it was suggested to switch to the IterativeAdaptive scheme, which has proven to be invaluable.

3) As will be justified in the mesh sensitivity study in Section 3, a change was made from first order, QUAD4 elements to second order QUAD8. This resolves a significant portion of the power discrepancy that can build up during depletion.

4) A cumulative damage index post-processor [17] has been added.

5) Various default values have been updated, such as those pertaining to densification and relocation.

An updated BISON template file is available in Appendix B.

\subsection{XML2MOOSE Preprocessor Updates}

Because the Watts Bar output data file for some cycles is a conglomeration of several output files as a result of necessary restarts, it was found that many of the data typically available (such as inputrelated data echoed in the HDF5 output) were not guaranteed to be available. For this reason, modifications were made to pull the data from the input XML files for each cycle. This should avoid future problems in which the HDF5 data may be inconsistent, depending on how the data files are consolidated.

The shuffling capability has also been extended to better account for quarter-core symmetry shuffling. Ongoing work is aiming to better optimize how this is performed, but at present, the baseline shuffling capability is sufficient to produce meaningful results.

\subsection{MOOSE/BISON/PETSc Updates}

Consultation with the BISON team regarding many of the issues encountered in the initial milestone helped clarify that several issues were related to the VERA MOOSE/BISON configuration being out of date. This prompted updates to the MOOSE and BISON repositories and an upgrade of the VERA PETSc version to 3.5.4. Additionally, while SuperLU cannot be distributed as part of the official VERA third party libraries (TPLs), several builds were generated that allow the SuperLU options within PETSc to be enabled, which is the solver option recommended by the BISON team. 


\subsection{Unit Testing Additions}

Two more unit tests to account for quarter symmetry shuffling with rotational (bison_from_vera_multi_qtr_shuffle_rot) and mirror (bison_from_vera_multi_qtr_shuffle_mir) symmetry have been added to the MOOSEExt repository. 


\section{WATTS BAR UNIT 1}

\subsection{General Description}

The Watts Bar Nuclear Plant is a Westinghouse four-loop PWR operated by the Tennessee Valley Authority (TVA) and is currently operating in its thirteenth cycle, logging over 6,000 effective full power days (EFPD) of operation since 1996 [1]. It began with a 3,411 megawatt thermal (MWth) power rating, but had a $1.4 \%$ power uprate in 2001 .

Figure 3.1.1 (left) shows a 2D slice of the WBN1 Cycle 1 full core layout, where the darker blue denotes moderator, lighter blue denotes $3.1 \%$ enriched rods, green denotes, $2.6 \%$ enriched rods, and red denotes $2.1 \%$ enriched rods. The structural components such as the core baffle, barrel, and pads are represented with a lighter gray whereas the vessel is shown with a darker gray. It has 193 Westinghouse $17 \times 17$ fuel assemblies which are 12 feet tall, each with 264 fuel rods and 25 guide/instrumentation tubes. On the right is a typical axial layout of a fuel assembly used in the nonproprietary model. It includes upper/lower core plate, nozzles, and gaps, with two Inconel and six Zircaloy spacer grids.
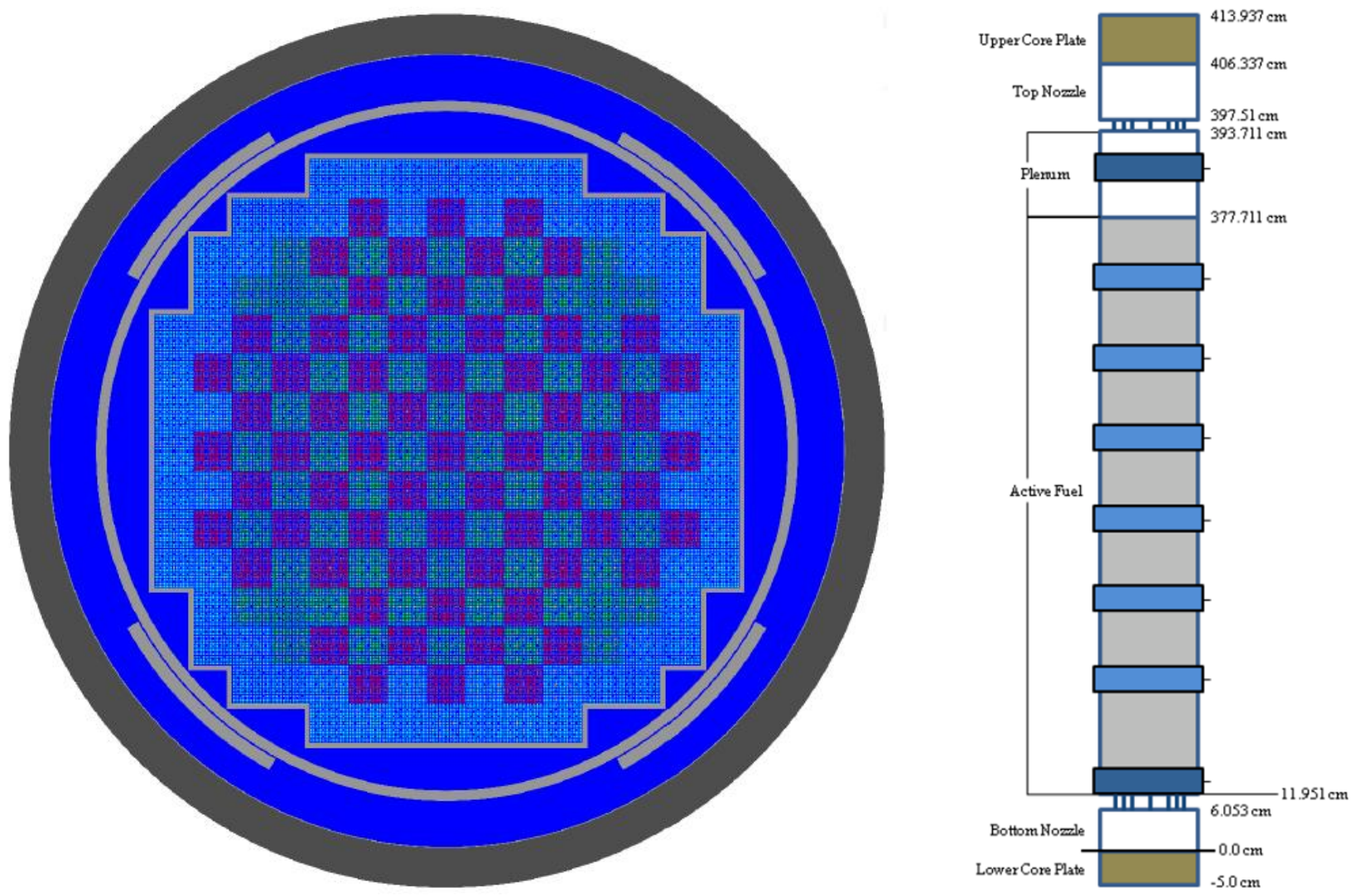

Fig. 3.1.1. Watts Bar Unit 1 - Cycle 1 Core diagram [1,18]

The left side of Fig. 3.1.2 shows a quarter core layout of the assembly configurations, including enrichment and the number of Pyrex rods for Cycle 1. The assemblies are color-coded based on the fuel enrichment. The right side of the figure shows the various control rod bank configurations, where operational banks are A-D and the safety banks at SA-SD, color-coded by bank. 

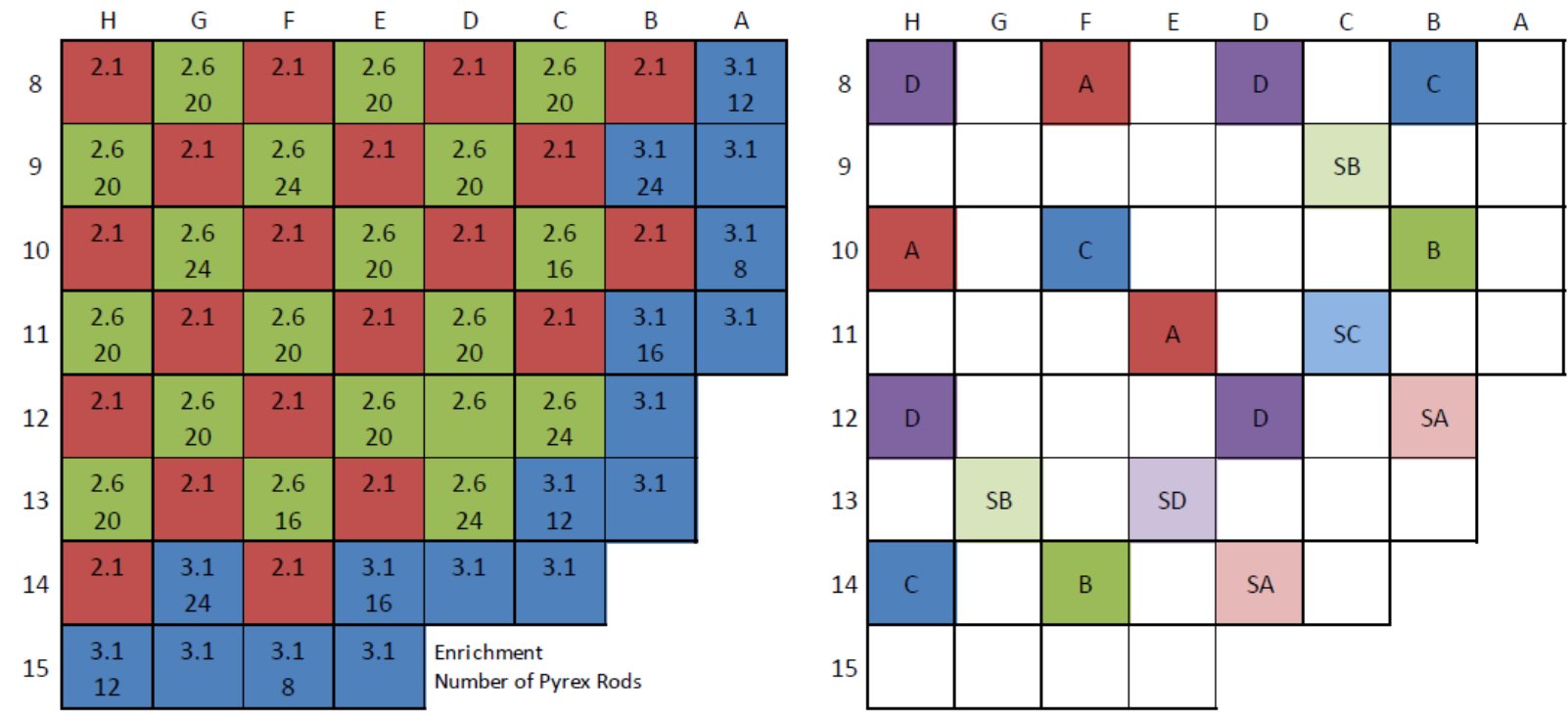

Fig. 3.1.2. Watts Bar Unit 1 - Cycle 1 core layout and rod bank configuration [1].

\subsection{Relevant Cycle Data}

To provide a brief insight into some of the more cycle-specific data, the following subsections show the core power history used in the VERA-CS cases, along with the core layout, as appropriate.

\subsubsection{Cycle 1}

Figure 3.2.1 shows the power history for Cycle 1, which has a more gradual ramp to power than will be seen in subsequent cycles as this is the first cycle of operation during which additional startup tests (including flux maps at stages of power ramp as are typical during any startup) are completed. There is also a bit more variation, though still relatively minor, than in other cycles. While this illustration is simpler than the real operational history, it is what was used in VERA-CS. Shortly after 14 gigawatt days per metric ton (GWd/MT), VERA-CS imposes a step change to $86.9 \%$ power. This was reflected in the BISON inputs, allowing a one-day transition to $86.9 \%$ during the downramp, and then after an additional $\sim 1 \mathrm{GWd} / \mathrm{MT}$ back up to $100 \%$ power since an instantaneous power change would be problematic for BISON. At all other statepoints, BISON uses a linear interpolation of the power. 


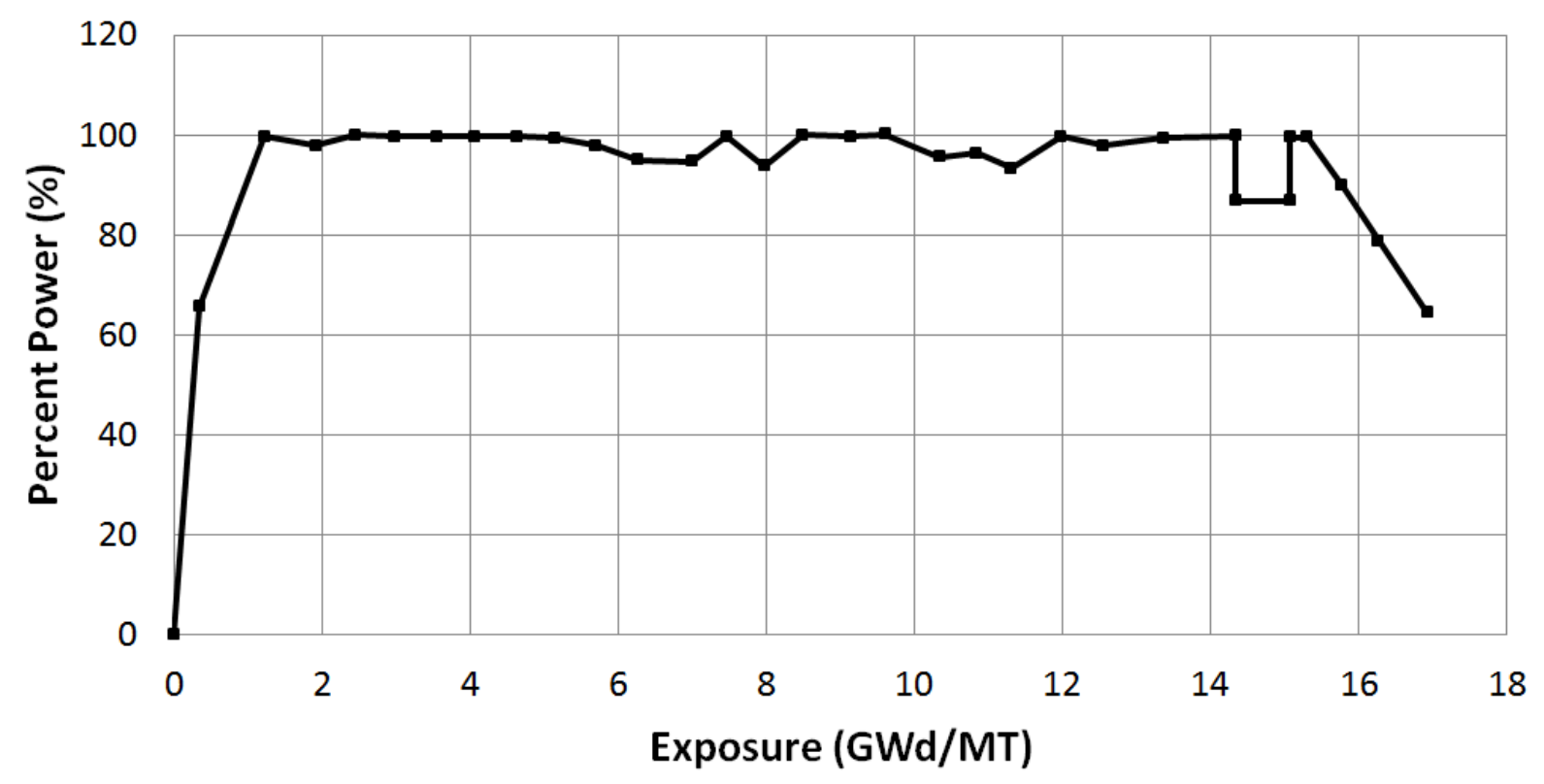

Fig. 3.2.1. Watts Bar Unit 1 - Cycle 1 power history $[1,19,20]$.

\subsubsection{Cycle 2}

Figure 3.2.2 shows the simulated power history for Cycle 2, which is very stable, with a more accelerated ramp to power, but with constant $100 \%$ power until a similar down-power ramp/coast down. The actual history was more complicated, including at least one trip.

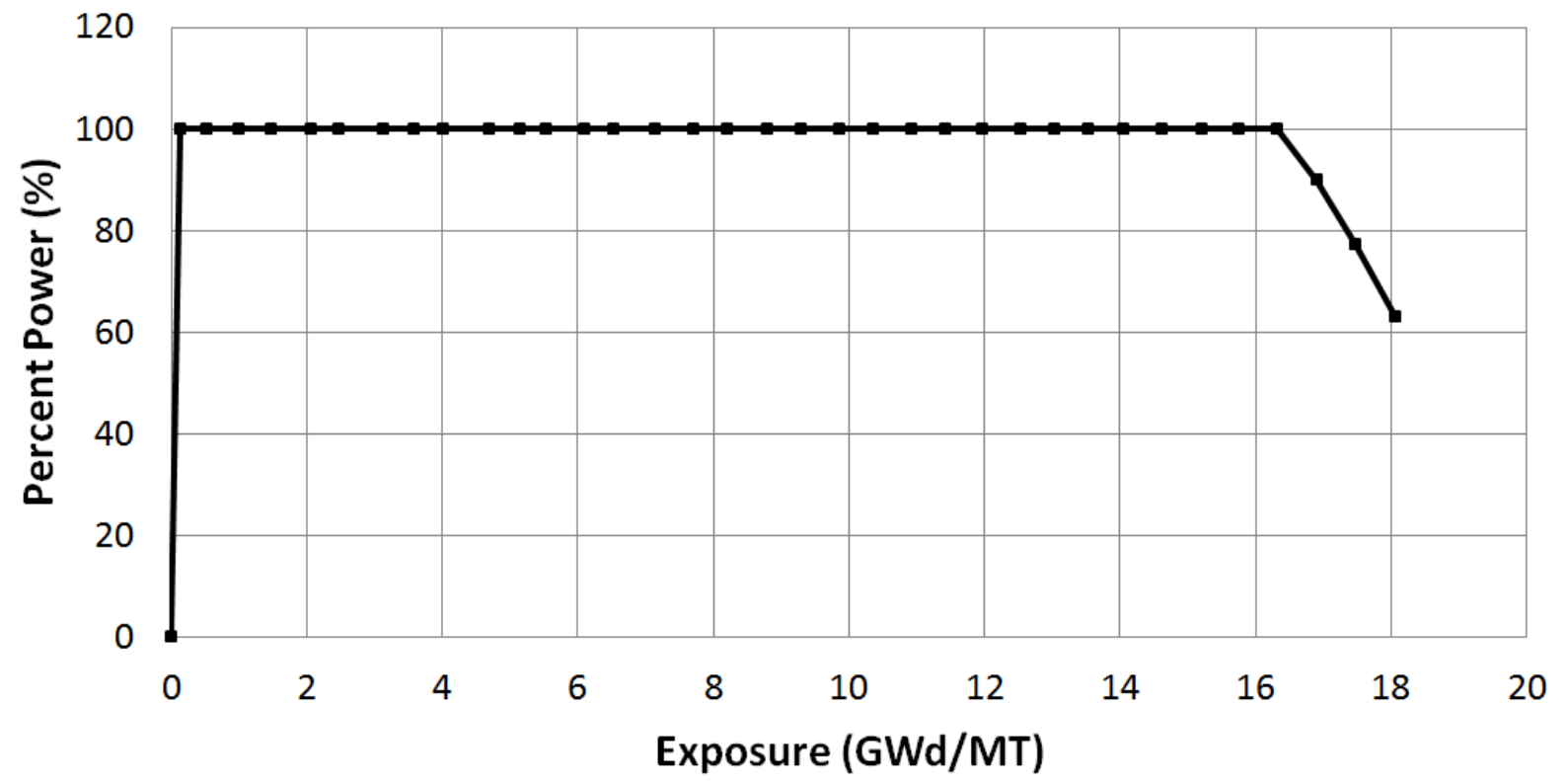

Fig. 3.2.2. Watts Bar Unit 1 - Cycle 2 power history $[1,21,22]$

Figure 3.2.3 shows the core layout in Cycle 2. Each assembly is color-coded based on enrichment, and fresh assemblies include data on the IFBA / wet annular burnable absorber (WABA) configuration, whereas others contain the corresponding location from Cycle 1. 


\begin{tabular}{|c|c|c|c|c|c|c|c|c|}
\hline $\mathrm{H}$ & G & $\mathrm{F}$ & $\mathrm{E}$ & D & C & B & A & \\
\hline 8 H-14 & $\mathrm{N}-13$ & $128^{*}$ & $\mathrm{R}-8$ & 128 & $N-8$ & L-15 & F-11 & \\
\hline $\mathrm{N}-3$ & 104 & $A-9$ & 104 | 8 & B-11 & $128^{*}$ & 48 & C-4 & \\
\hline $0128 *$ & G-15 & $\mathrm{E}-15$ & D-7 & 104 | 8 & B-7 & 48 & G-10 & \\
\hline $\mathrm{H}-1$ & $104 \mid 8$ & $\mathrm{~J}-12$ & $128^{*}$ & $\mathrm{~N}-2$ & 128 & 48 & $F-13$ & \\
\hline 128 & $\mathrm{E}-14$ & 104 | 8 & $P-3$ & $A-6$ & $104 \mid 4$ & B-4 & & Batch 1 - 2.11\% \\
\hline $\mathrm{H}-3$ & $128^{*}$ & $\mathrm{~J}-14$ & 128 & $104 \mid 4$ & & $P-6$ & & Batch $2-2.619 \%$ \\
\hline R-5 & 48 & 48 & 48 & M-14 & $\mathrm{K}-2$ & & & Batch 3 - 3.1\% \\
\hline L-10 & M-13 & F-9 & C-10 & $\begin{array}{l}\text { IFBA | W } \\
\text { Previous }\end{array}$ & $\begin{array}{l}\text { ABA or } \\
\text { Cycle } 1\end{array}$ & cation & & Batch $4-3.709 \%$ \\
\hline
\end{tabular}

Fig. 3.2.3. Watts Bar Unit 1 - Cycle 2 core layout [18].

\subsubsection{Cycle 3}

Figure 3.2.4 shows the simulated power history for Cycle 3, which is very similar to Cycle 2, and Fig. 3.2.5 shows the core layout in Cycle 3.

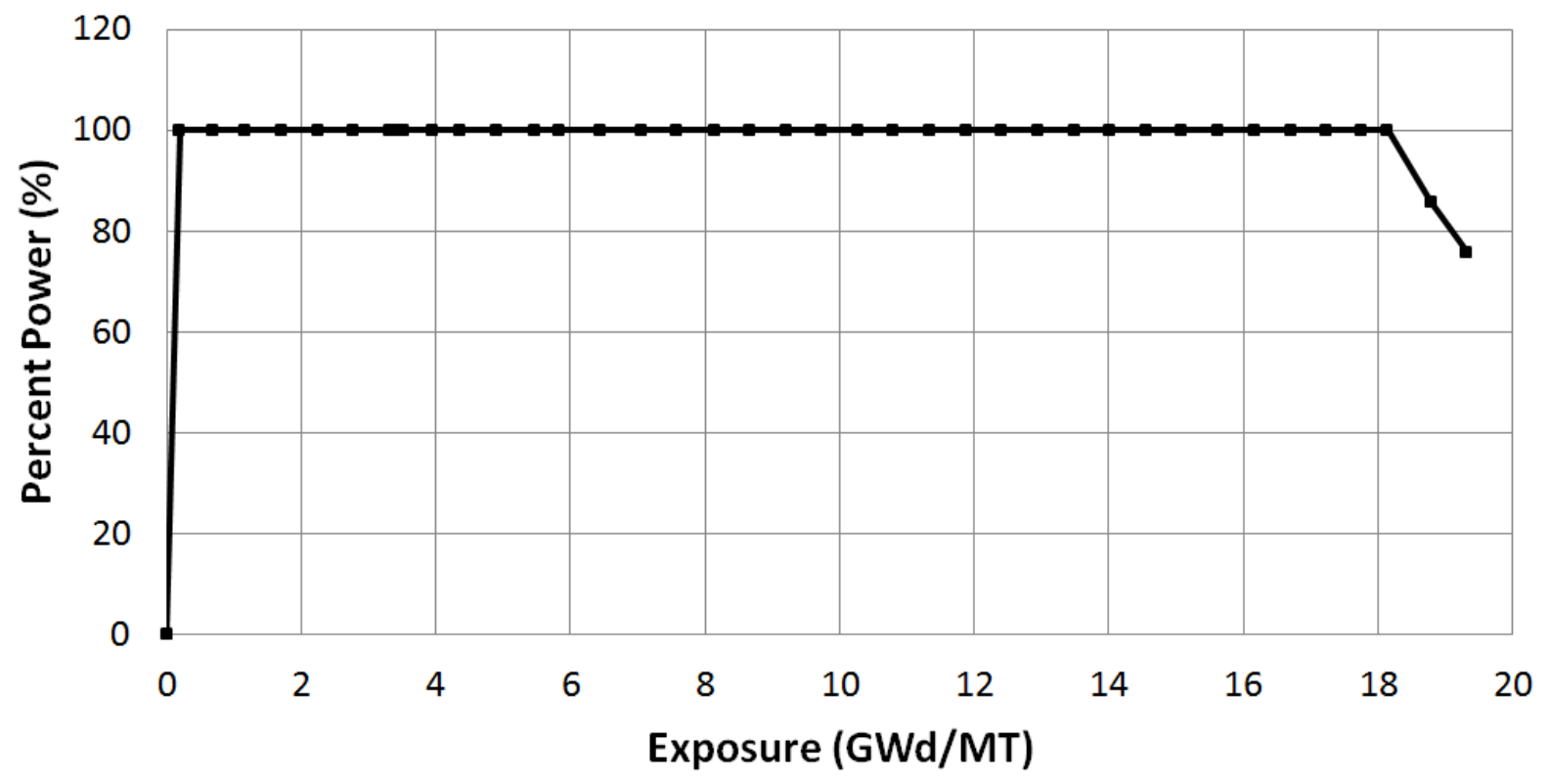

Fig. 3.2.4. Watts Bar Unit 1 - Cycle 3 power history $[1,23,24]$. 


\begin{tabular}{|c|c|c|c|c|c|c|c|c|c|}
\hline & $\mathrm{H}$ & G & $\mathrm{F}$ & $E$ & D & C & B & A & \\
\hline 8 & $1 \mathrm{~A}-10$ & $\mathrm{H}-6$ & 128 & $L-5$ & $\mathrm{~N}-13$ & $D-8$ & 104 & $J-9$ & \\
\hline 9 & $F-8$ & $128 \mid 8$ & D-13 & $128 \mid 8$ & B-7 & 104 & 128 & $M-10$ & \\
\hline 10 & 128 & $C-12$ & L-2 & $N-11$ & 128 & G-11 & 128 & $\mathrm{M}-2$ & Batch 1 - 2.11\% \\
\hline 11 & $E-5$ & $128 \mid 8$ & $E-3$ & 128 & $J-3$ & 128 & 16 & $A-5$ & Batch 2 - 2.619\% \\
\hline 12 & $\mathrm{~N}-3$ & J-14 & 128 & $N-7$ & E-2 & 128 & P-10 & & Batch $3-3.1 \%$ \\
\hline 13 & $\mathrm{H}-12$ & 104 & E-9 & 128 & 128 & 16 & G-15 & & Batch $4-3.709 \%$ \\
\hline 14 & 104 & 128 & 128 & 16 & $\mathrm{~F}-2$ & A-9 & & & Batch $5 A-3.807 \%$ \\
\hline 15 & J-7 & $\mathrm{F}-4$ & $P-4$ & L-15 & $\begin{array}{l}\text { IFBA | } \\
\text { Previo }\end{array}$ & $\begin{array}{l}\text { ABA or } \\
\text { Cycle L }\end{array}$ & ation & & Batch 5B - 4.401\% \\
\hline
\end{tabular}

Fig. 3.2.5. Watts Bar Unit 1 - Cycle 3 core layout $[23,24]$.

\subsection{Remarks}

It is important to note that BISON does not have the capability to simulate the IFBA coating on fuel pellets. This coating will affect the fuel performance physics, particularly the internal rod pressure. The results in this work neglect the IFBA coating, but a model to account for the coating is actively being developed at INL, and these results will be regenerated once it becomes available. Additionally, many IFBA rods use a combination of solid and annular fuel pellets (annular pellets at the axial extremities) to accommodate the additional helium generated by the IFBA coating. At present, BISON does not have an internal mesh capability that can accommodate this case, so annular pellets are also being represented as solid. The capability to do this is also actively under development, but it is worth noting that these pellets are at very low power anyway, and are likely not limiting in any way.

The BISON cases in this work used Zircaloy-4 material models as opposed to proprietary ZIRLO material models that would be necessary in these rods. Additionally, the oxide layer growth is only available in select boundary condition modes of BISON, which is not one used in this work. Extending the oxide layer growth capability (waterside and internal) is planned for future work by the BISON development team. 


\section{MESH SENSITIVITY STUDY}

As work with BISON progressed, it became clear that an independent mesh sensitivity study would be beneficial to ensure that the mesh parameters being used were sufficiently tight for this application. An internal report on INL's similar study [25] highlighted some potential issues with using a coarse mesh, particularly in the discrepancy between input and calculated rod power. In this study, eight different configurations were tested: four different mesh options (all equi-spaced) and two different quadrature options. These configurations were tested on a single rod from WBN1 Cycle 1 that was run on 12 processors on Falcon.

\subsection{Mesh and Quadrature Description}

Table 4.2.1 shows the mesh options that were used. Because the aspect ratio of each element is important to convergence (with the target in BISON of around 20 [25]), each of these meshes preserves the same aspect ratio, so if the radial mesh is doubled, so is the axial mesh.

Table 4.2.1. Fuel mesh parameters

\begin{tabular}{ccc}
\hline & \# Radial Mesh & \# Axial Mesh \\
\hline coarse & 6 & 100 \\
medium & 12 & 200 \\
fine & 24 & 400 \\
ultrafine & 48 & 800 \\
\hline
\end{tabular}

Two different quadrature options were assessed: (1) linear, consisting of QUAD4 and first order elements, and (2) quad, consisting of QUAD8 and second order element options.

\subsection{Results}

Figures 4.2.1-4.2.3 show the maximum centerline fuel temperature, average fuel temperature, and minimum gap thickness for each of the eight different options. In the temperature plots, all but the coarse linear configuration agree well. All agree well for the gap thickness estimation.

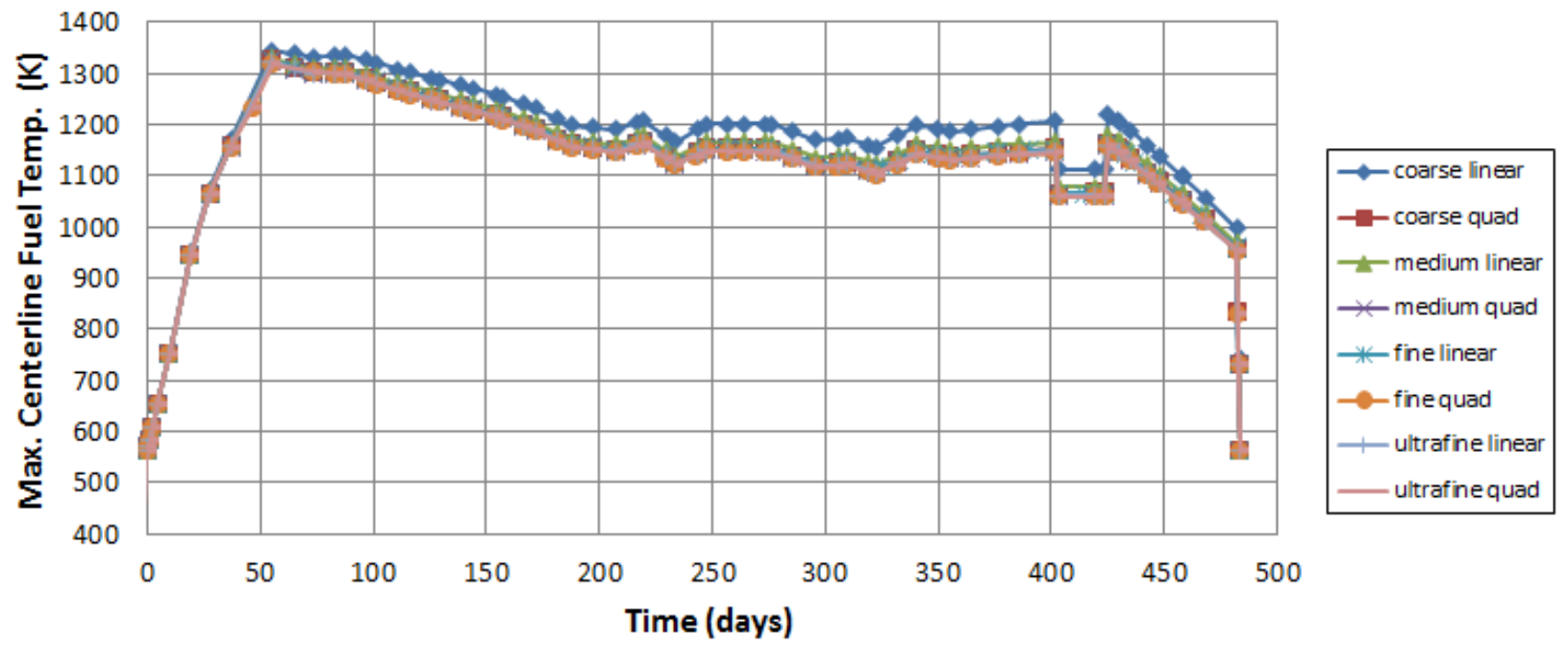

Fig. 4.2.1. Mesh sensitivity study - maximum centerline fuel temperature (K). 


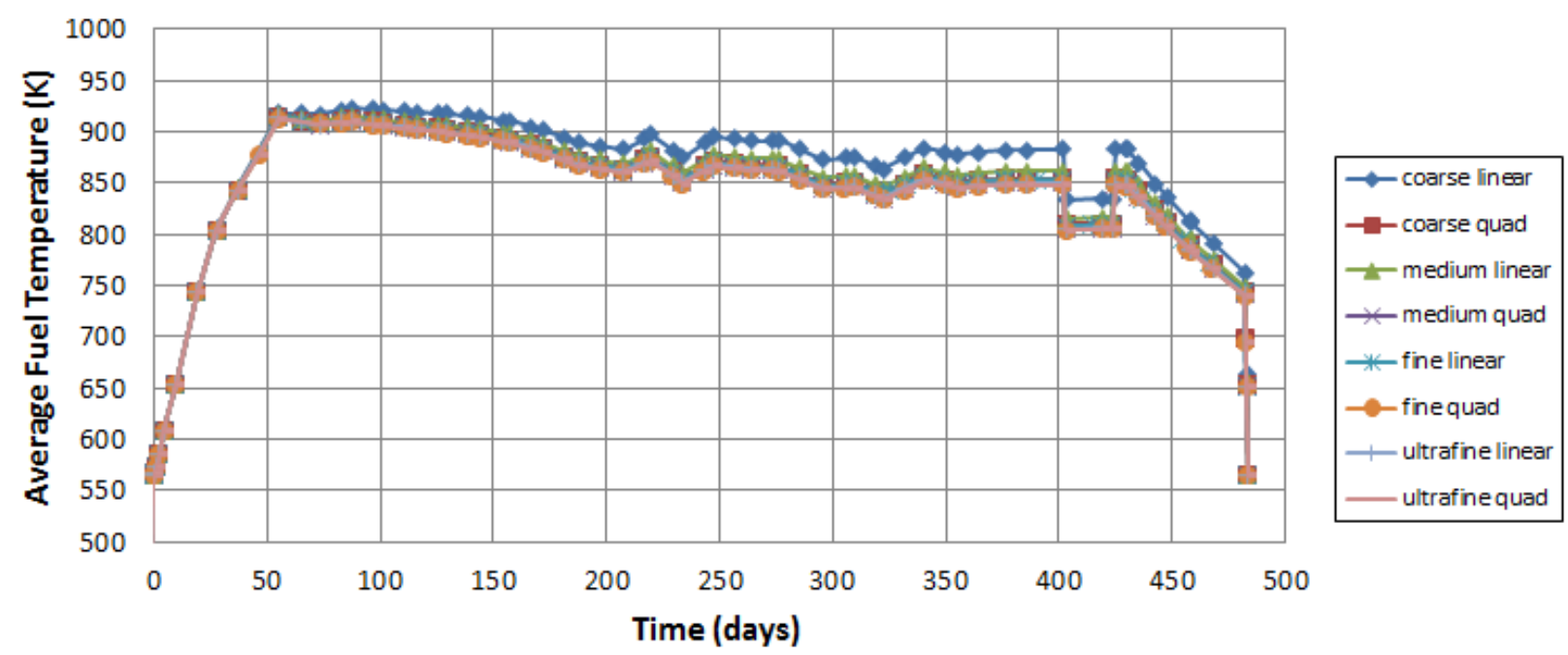

Fig. 4.2.2. Mesh sensitivity study - average fuel temperature (K).

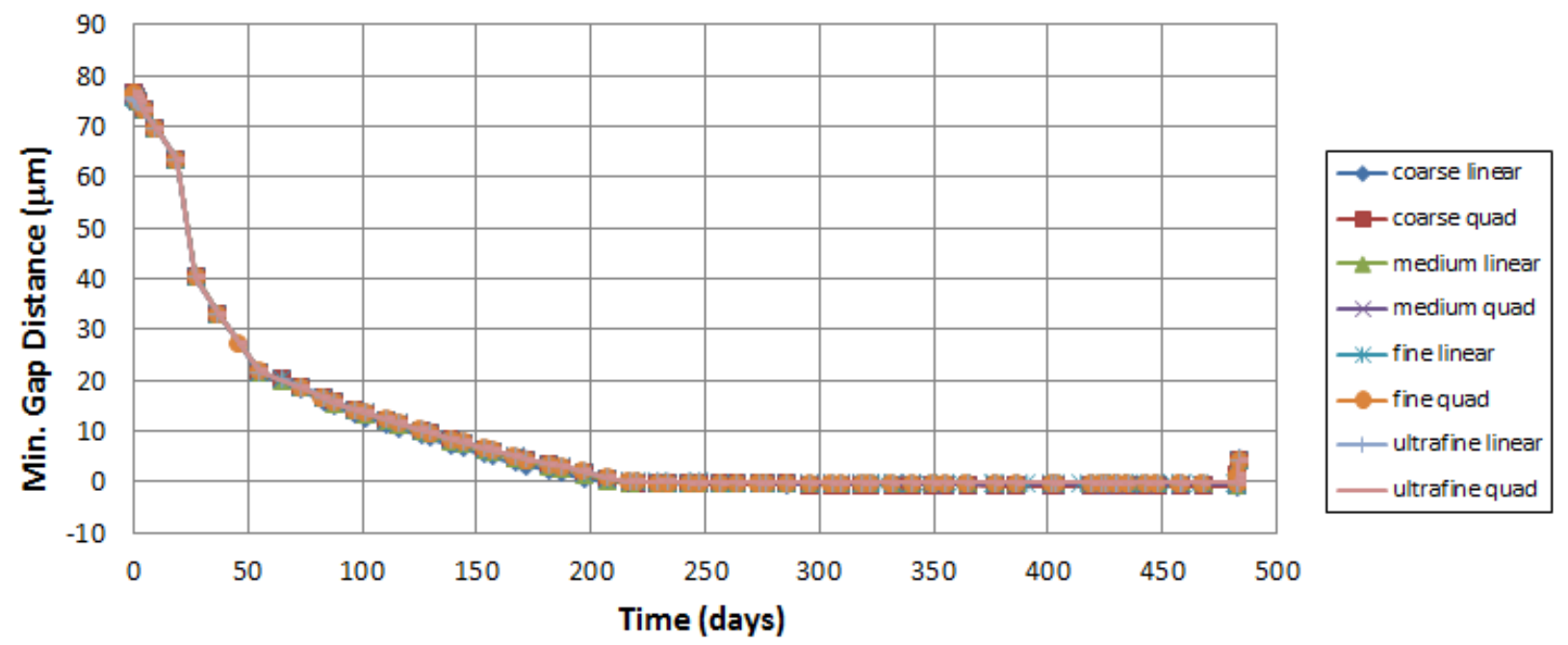

Fig. 4.2.3. Mesh sensitivity study - minimum gap thickness $(\mu \mathrm{m})$.

However, for the difference between input (Watts) and calculated power (Watts), more substantial differences are observed, with coarse linear yielding at nearly a $20 \%$ difference by the end of the cycle. While the rod power history is specified as an input to BISON, various components of the solve can lead to a lack of conservation of power in the sense that the power calculated from the BISON mesh is not guaranteed to match the specified power exactly. Most of this discrepancy is well resolved with fine linear or medium quad. The radial mesh used here is equispaced. The same internal INL report [17] showed that a non-uniform biased mesh can significantly reduce this discrepancy even further. While the biased meshing option is currently not available with BISON's internal mesh generator, it is under development and will be a useful addition. 


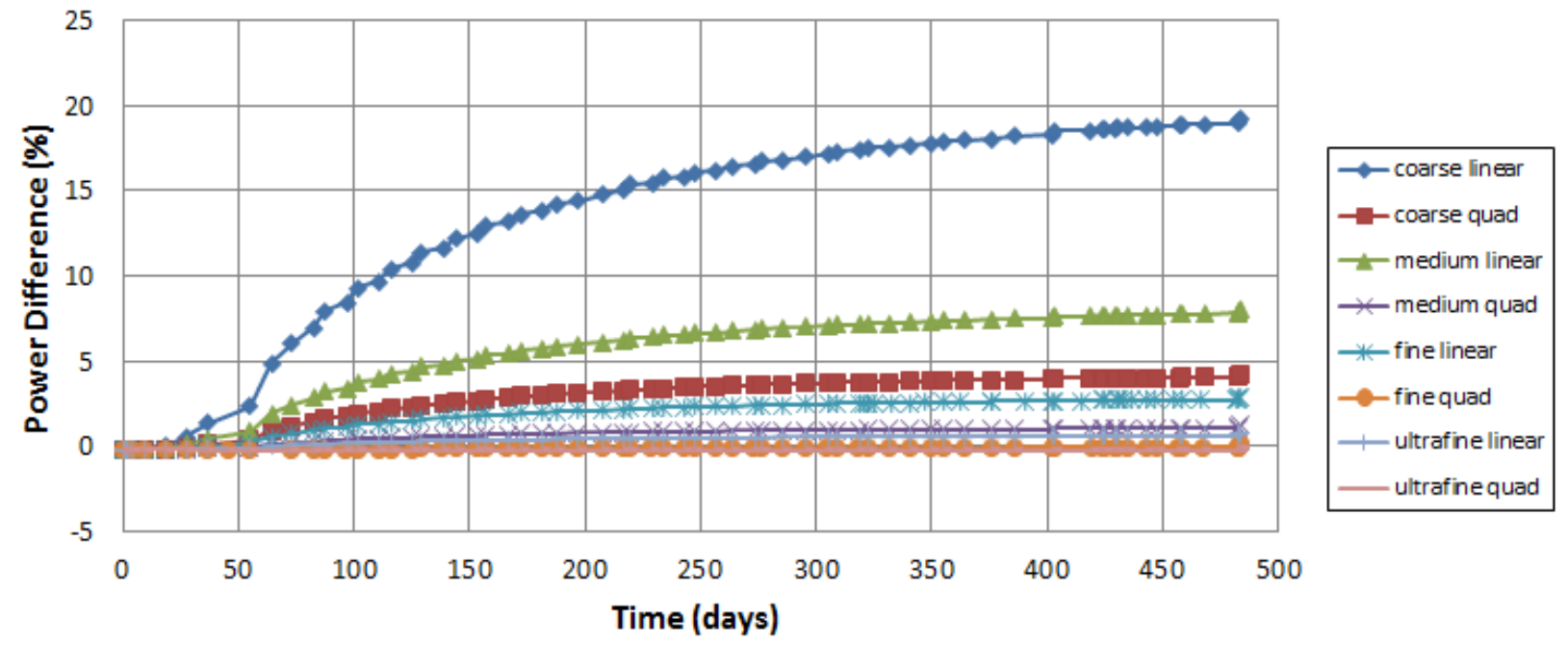

Fig. 4.2.4. Mesh sensitivity study - power difference (\%).

Table 4.2.2 shows the timing results for the configurations. In general, going from linear to quad for a particular mesh refinement is more cost effective than doubling the mesh.

Table 4.2.2. Timing results for various meshes

\begin{tabular}{ccc}
\hline & \multicolumn{2}{c}{ Time (min) } \\
\cline { 2 - 3 } & linear & \multicolumn{1}{c}{ quad } \\
\hline coarse & 2.05 & 4.73 \\
medium & 5.23 & 12.30 \\
fine & 17.58 & 43.48 \\
ultrafine & 59.73 & 179.12 \\
\hline
\end{tabular}

For this reason, the coarse quad parameters were used in the remainder of the analysis and in this report since it (1) has a much improved discrepancy over coarse linear, (2) provides greater improvement than medium linear mesh with less computational burden, and (3) will likely be even better once biased meshing options are available, providing performance similar to that anticipated in the long-term option. 


\section{RESULTS}

The available output data is briefly summarized here, and then the results for the first and last full power data points (near-BOC and near-EOC) of each cycle are presented for the following parameters of interest:

1) maximum centerline fuel temperature,

2) average fuel temperature,

3) minimum gap thickness,

4) minimum clad hoop stress, and

5) maximum clad hoop stress.

In addition, a more complete set of data for the maximum centerline fuel temperature and minimum gap thickness for each cycle is available in Appendix A. The results in this section and the appendix are shown at burnups that were used as VERA-CS statepoints. True BOC and true EOC cycle results for most parameters were omitted because they are at low or zero power. However, Appendix $\mathrm{A}$ includes $\mathrm{BOC} / \mathrm{EOC}$ for the maximum centerline fuel temperature and minimum gap thickness.

To run these cases, a large number of 24-rod batch jobs were executed on Falcon on 12 processors each. This is primarily because the means to run all rods at once (such as MultiApps) is not yet available. To complete these jobs, a total of approximately 74,000 core-hours were used. Previous discrepancies reported in parallel execution of BISON in an earlier milestone report [15] have since been resolved, and consistency is observed.

\subsection{Output Data}

A variety of data is available from BISON in (1) comma-separated value (CSV) files, which typically contain maximum, minimum, and average values of quantities of interest (temperatures, stress, etc.) and (2) EXODUS files, which can contain higher resolution data such as axial and finite element-based distributions. At this point, only data from CSV files are currently accessible in the post-processing tool that consolidates that output data onto VERAOut. Work is underway to gather the data from the EXODUS files.

\subsection{Cycle 1}

Figures 5.2.1-5.2.5 show the results for several parameters of interest for Cycle 1. Figure 5.2.1 shows the maximum centerline fuel temperature, which peaks at roughly $1516 \mathrm{~K}$ (much lower than the melting temperature of $\mathrm{UO}_{2}$ ). Not surprisingly, it is considerably higher early in the cycle and decreases as fuel densification and gap closure help bring it down as the cycle progresses. The distribution also flattens out along with the power profile as the higher enriched assemblies burn out more rapidly. 

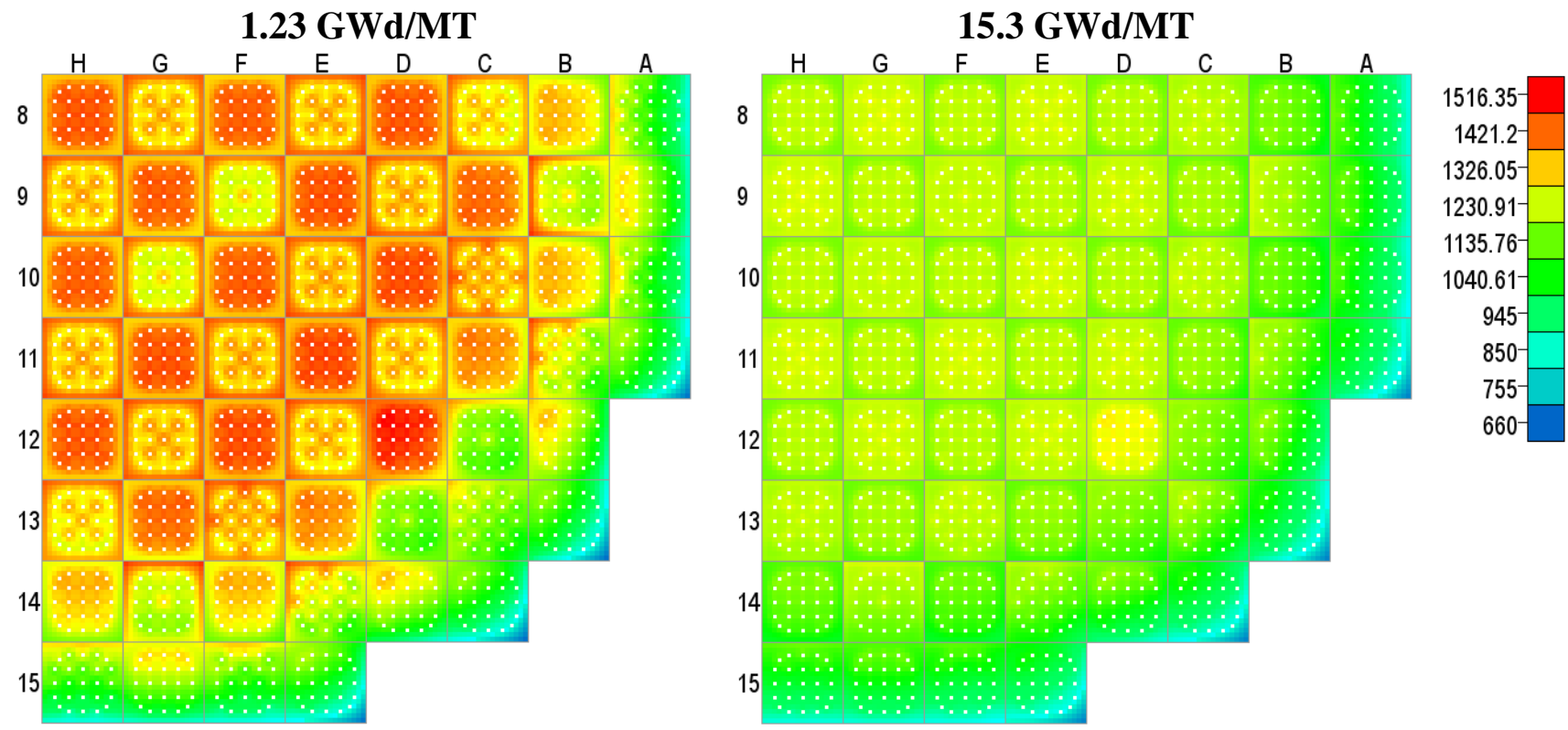

Fig. 5.2.1. Cycle 1 maximum centerline fuel temperature (K).

Figure 5.2.2 shows the average fuel temperature, which exhibits similar trends as observed in the maximum centerline fuel temperature.
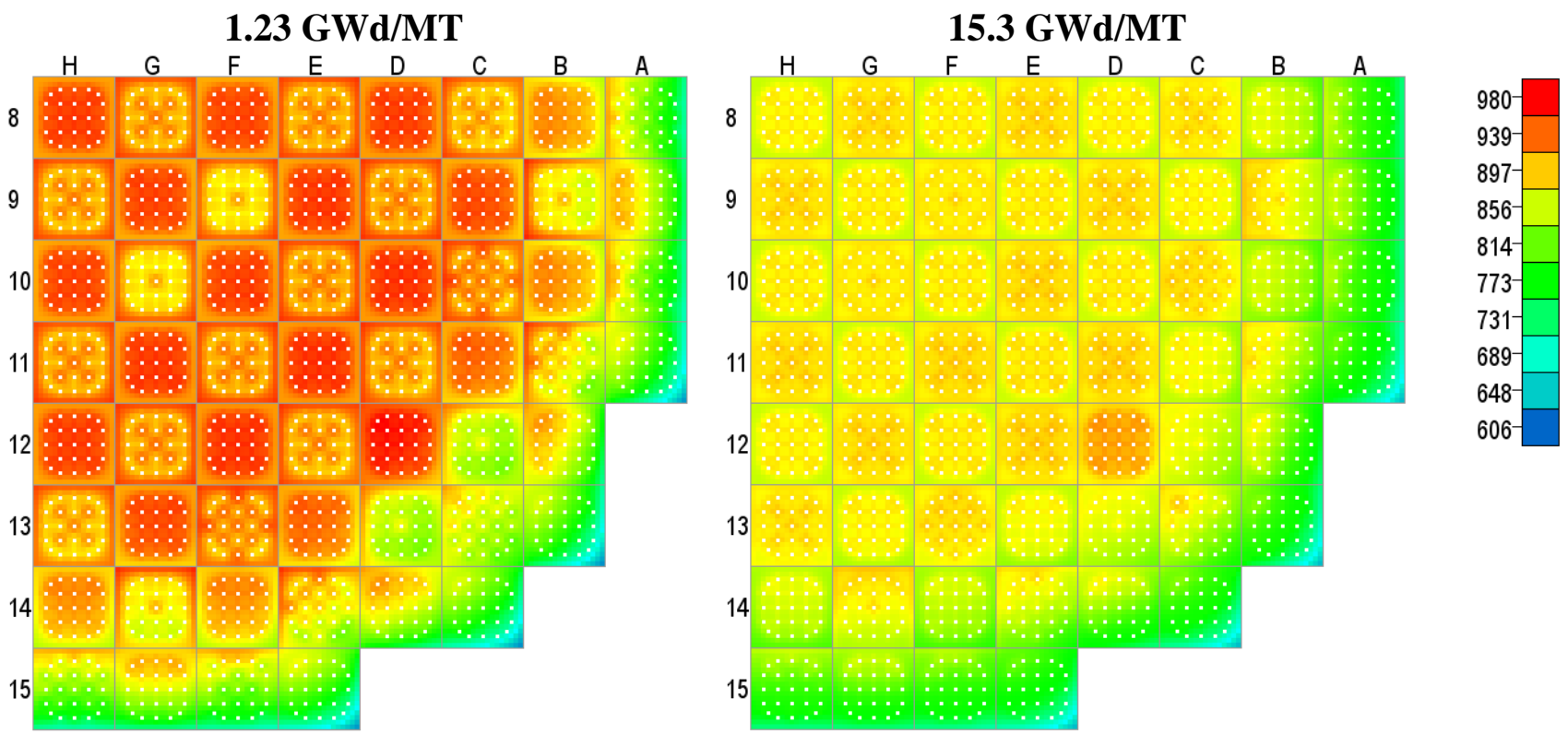

Fig. 5.2.2. Cycle 1 average fuel temperature (K). 
Figure 5.2.3 shows the minimum gap thickness for each rod, where it can be seen that none of the rods are in contact near BOC (as expected), but as the cycle progresses, nearly all rods have some contact by the end of the cycle.
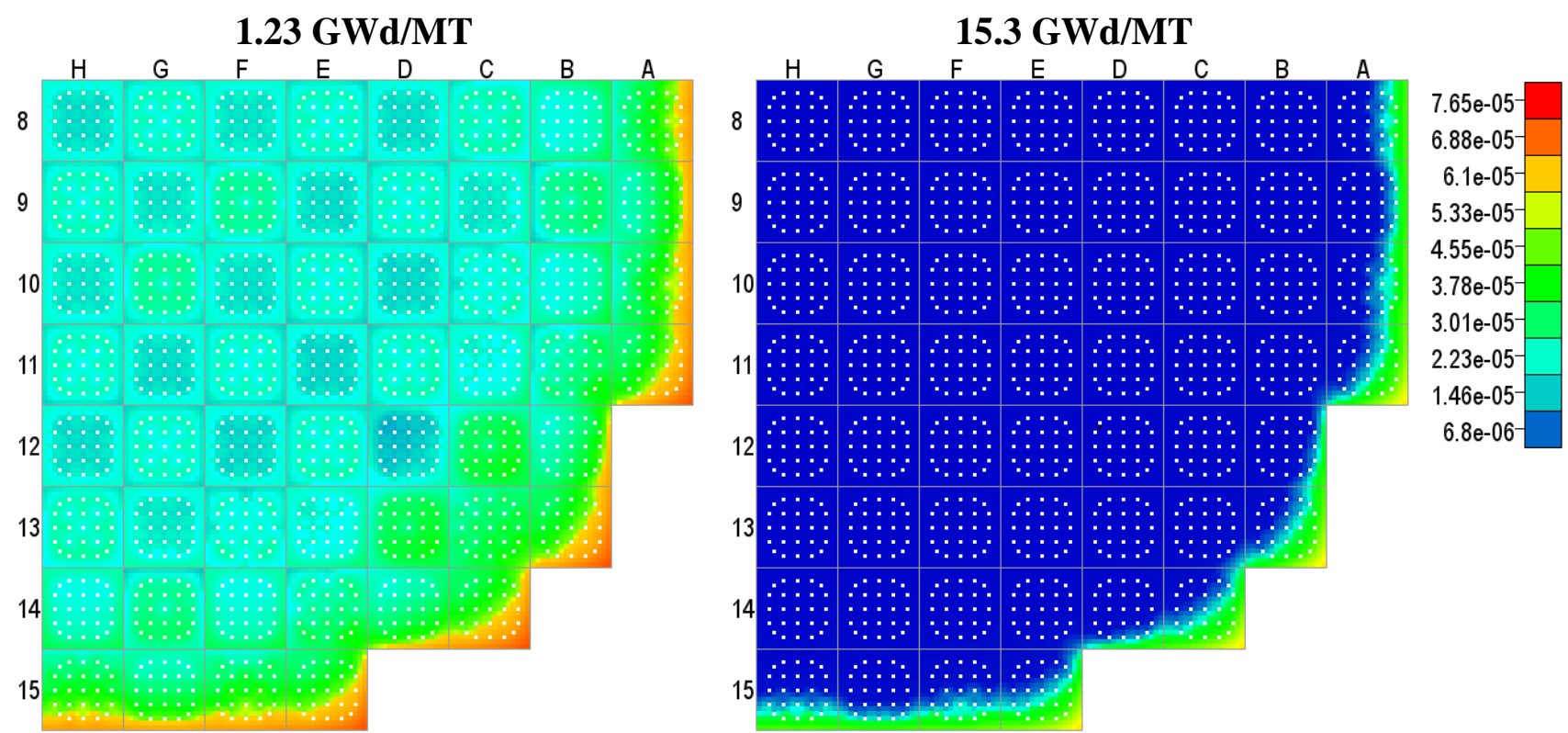

Fig. 5.2.3. Cycle 1 minimum gap thickness (m).

Figure 5.2.3 shows the minimum clad hoop stress. There is very little interesting about these figures, but worth watching in future work.

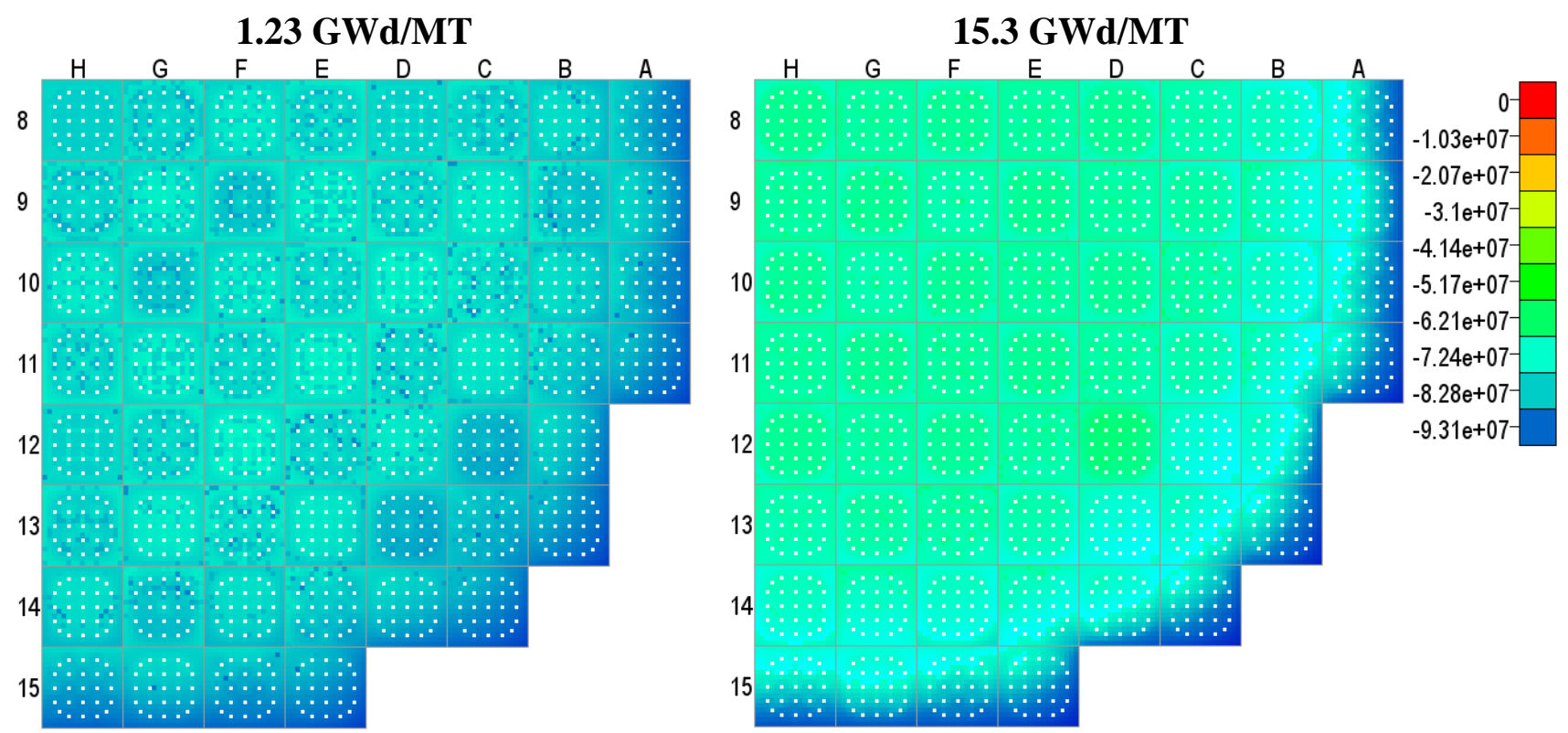

Fig. 5.2.4. Cycle 1 minimum clad hoop stress (Pa).

Figure 5.2.4 shows the maximum clad hoop stress. The spike near the end of the cycle seems to result from the power transition to/from $86.9 \%$ power near the end of the cycle, which also includes a small movement of the Bank D rods, which are located at H-8, D-8, H-12, and D-12 and clearly stand out near EOC. However, these stresses ( 30 MPa) are still low and are not very concerning. 

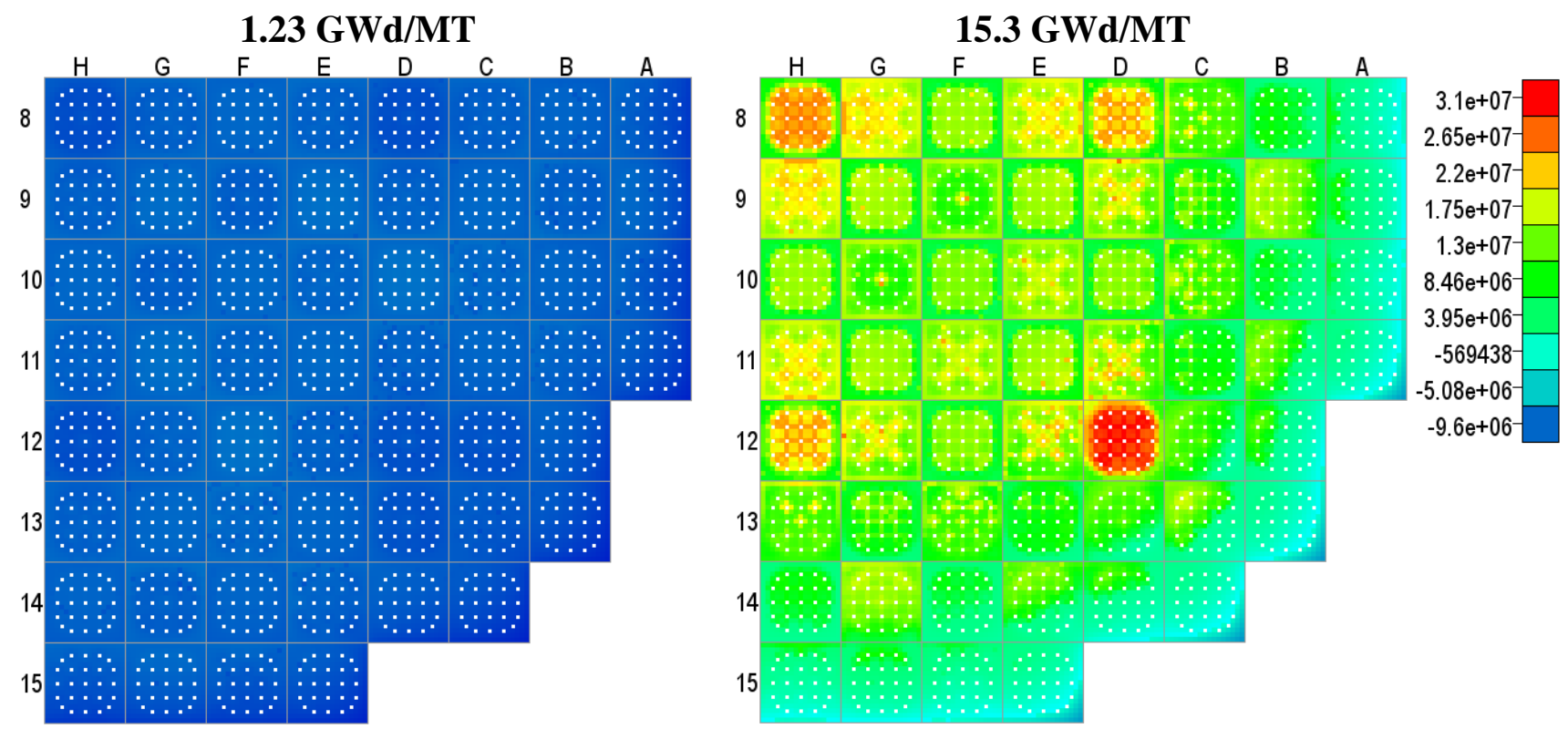

Fig. 5.2.5. Cycle 1 maximum clad hoop stress (Pa).

\subsection{Cycle 2}

Figures 5.3.1-5.3.5 show results for several parameters of interest for Cycle 2. Based on the results, it is easy to identify the fresh and burned assemblies at beginning of cycle (BOC). For example, in Figures 5.3.1 and 5.3.2, the fresh assemblies have higher temperatures because the enrichments (and resultantly power) are higher. It can also been seen in Figure 5.3.3 as most of the burned assemblies have a substantial number of rods with contact at BOC, whereas the fresh assemblies have no contact present yet. Of the several thousand rods displayed, a couple encountered convergence problems, which seem to arise during cycle outages where the power is zero. However, this is a significant improvement over the results in the previous milestone [15], in which two rods out of several hundred had issues. In these plots, troubled rods show up just as guide tubes do, with zero value entries. For example, looking at $\mathrm{H}-15$, there is one additional zero entry in the lower right quadrant.

Figures 5.3.1 and 5.3.2 show the maximum centerline and average fuel temperature distribution. Very few surprises and all temperatures are well below any cause for concern. 

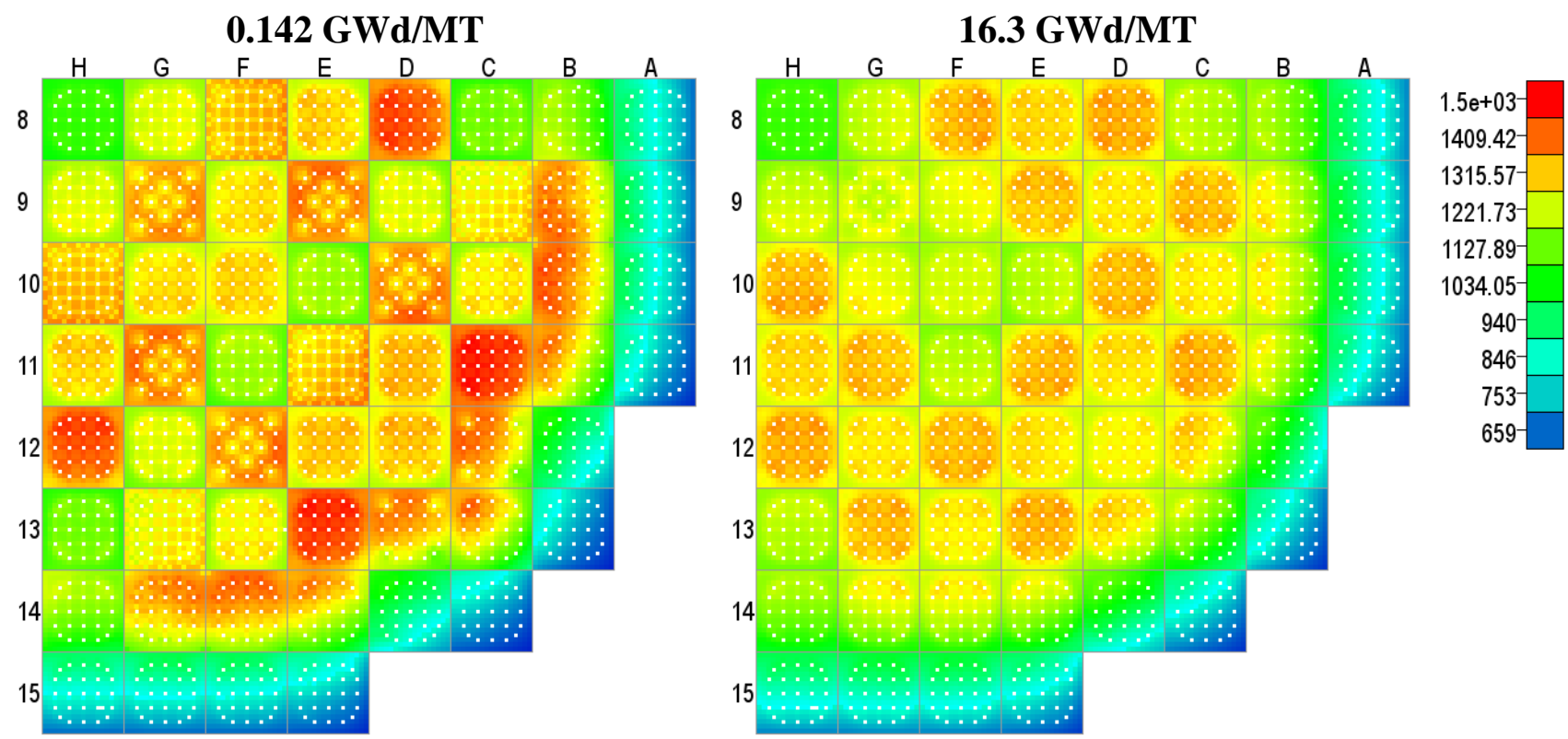

Fig. 5.3.1. Cycle 2 maximum centerline fuel temperature (K).
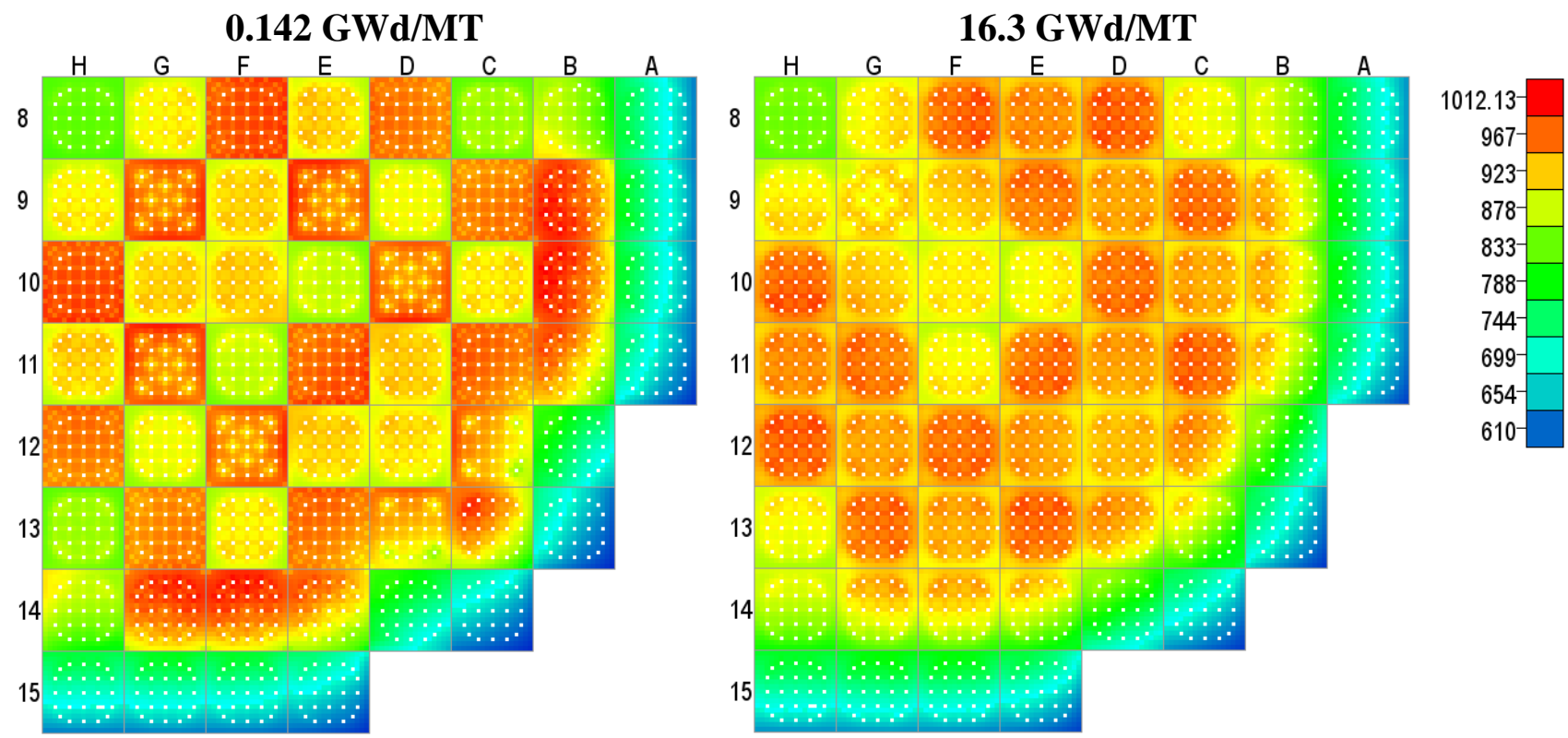

Fig. 5.3.2. Cycle 2 average fuel temperature (K). 
Figure 5.3.3 show the minimum gap thickness. As in Cycle 1, the fresh assemblies do not exhibit any fuel-clad contact early in the cycle, though nearly all pins do by EOC.
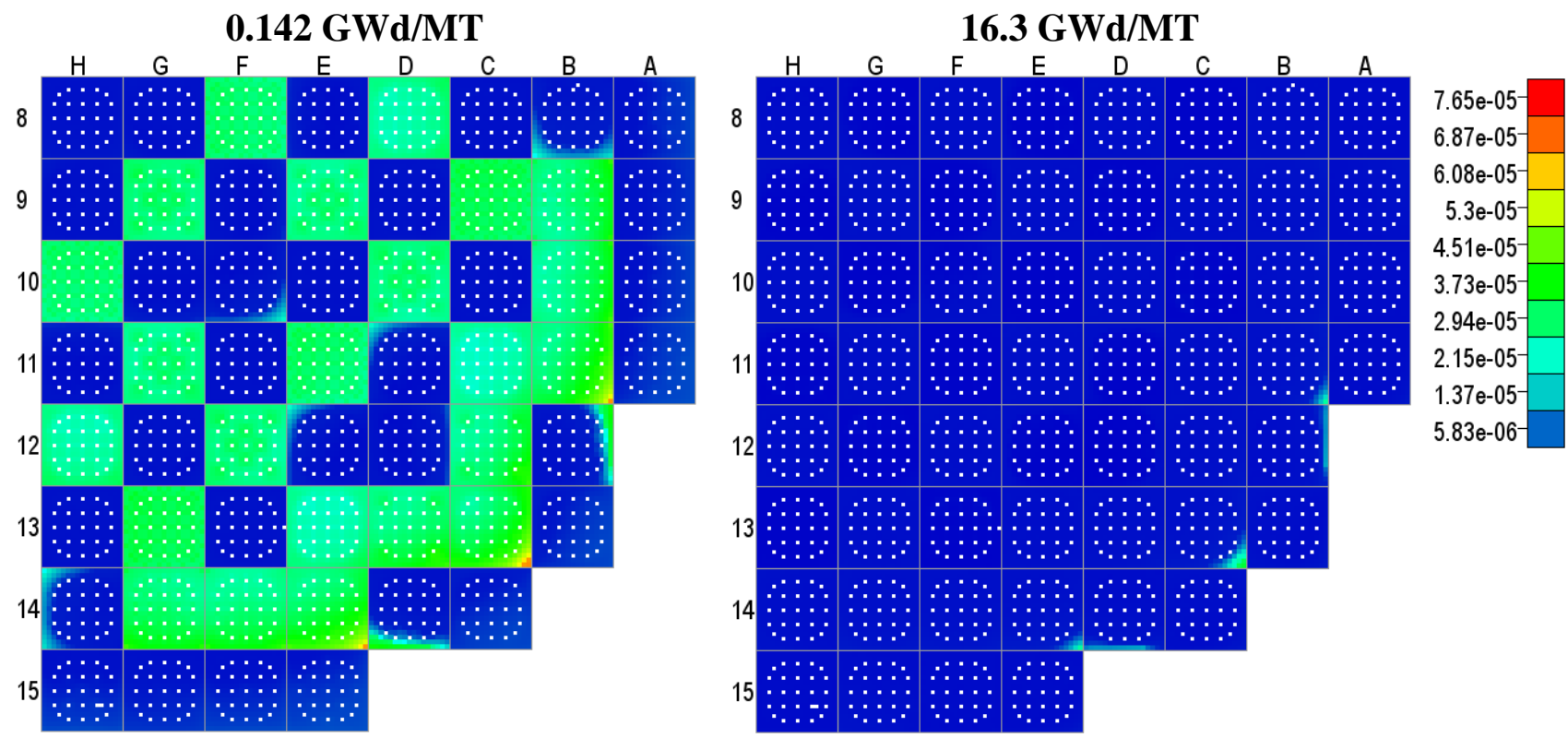

Fig. 5.3.3. Cycle 2 minimum gap thickness (m).
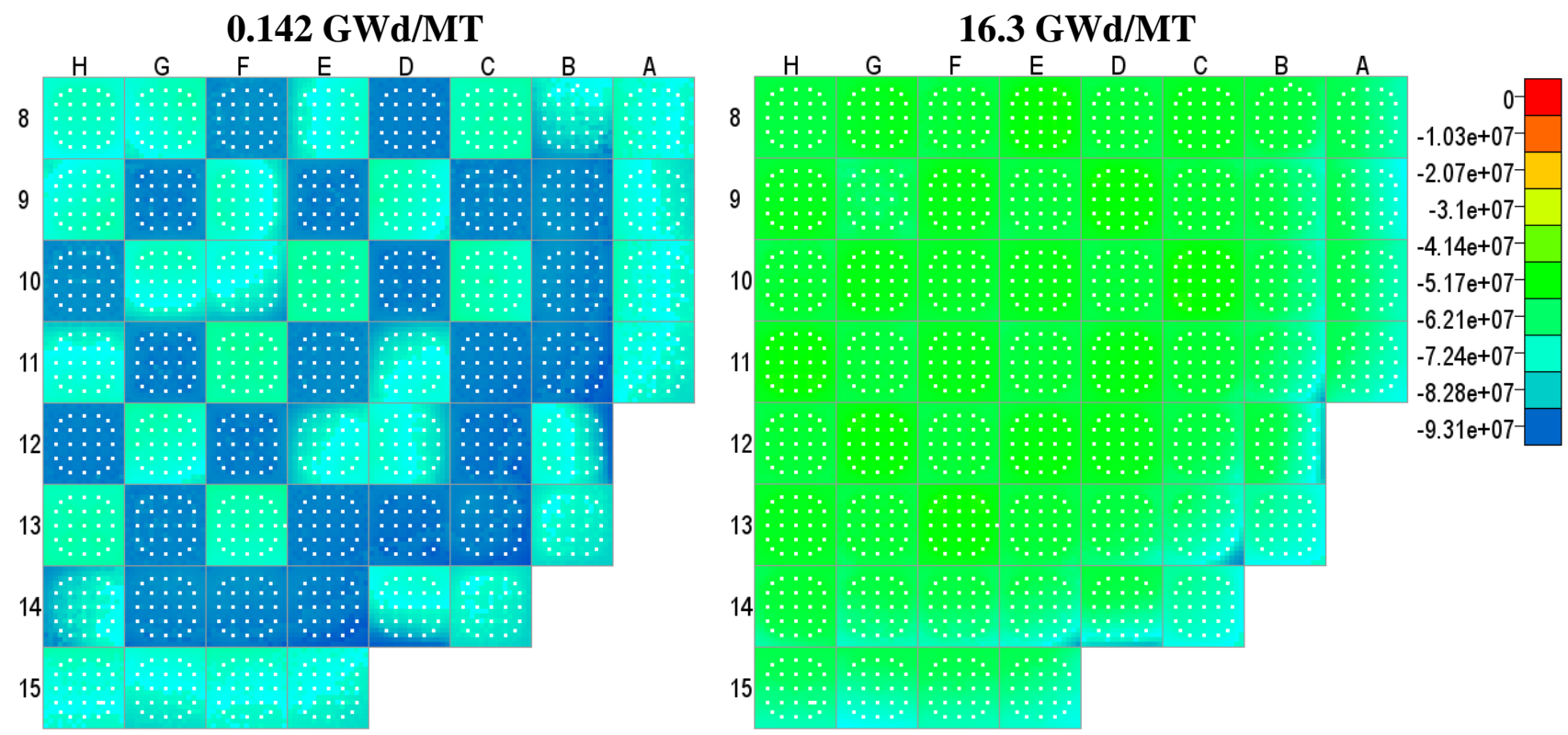

Fig. 5.3.4. Cycle 2 minimum clad hoop stress (Pa). 
Figure 5.3.5 shows the maximum hoop stress distribution for Cycle 2. The maximum hoop stresses are considerably higher than in Cycle 1 at roughly $250 \mathrm{MPa}$. Much of this spike is related to the ramp to power, which is much quicker than in Cycle 1. Based on the VERA-CS power input, the transition happened over about 7 days. However, as the cycle progresses, the stresses reduce considerably.
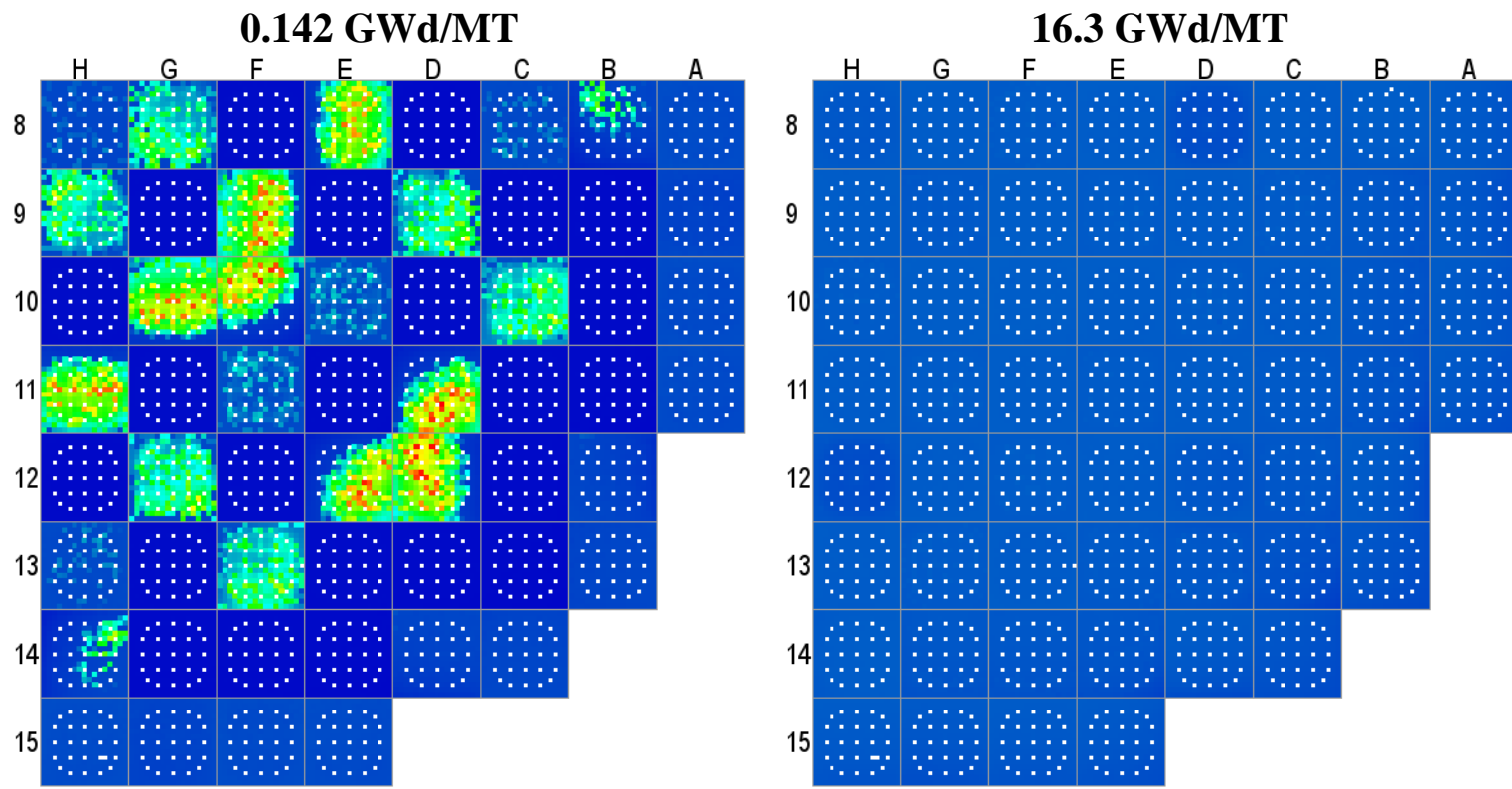

$2.54 \mathrm{e}+08$
$2.27 \mathrm{e}+08-$
$2 \mathrm{e}+08-$
$1.73 \mathrm{e}+08-$
$1.47 \mathrm{e}+08-$
$1.2 \mathrm{e}+08-$
$9.31 \mathrm{e}+07-$
$6.64 \mathrm{e}+07-$
$3.97 \mathrm{e}+07-$
$1.3 \mathrm{e}+07-$

Fig. 5.3.5. Cycle 2 maximum clad hoop stress (Pa).

Figure 5.3.6 shows the cumulative damage index for the cycle. Interestingly, no CDI is registered at the $0.142 \mathrm{GWd} / \mathrm{MT}$ statepoint, which is the first full power statepoint, but is registered at the next statepoint $(0.521 \mathrm{GWd} / \mathrm{MT})$, as shown here. The CDI distribution tends to correspond well with the maximum clad hoop stress in the previous figure, which is a major component in the CDI equation [17]. Based on this, the maximum CDI is around $4 \%$ for a couple of rods, which is still relatively low.
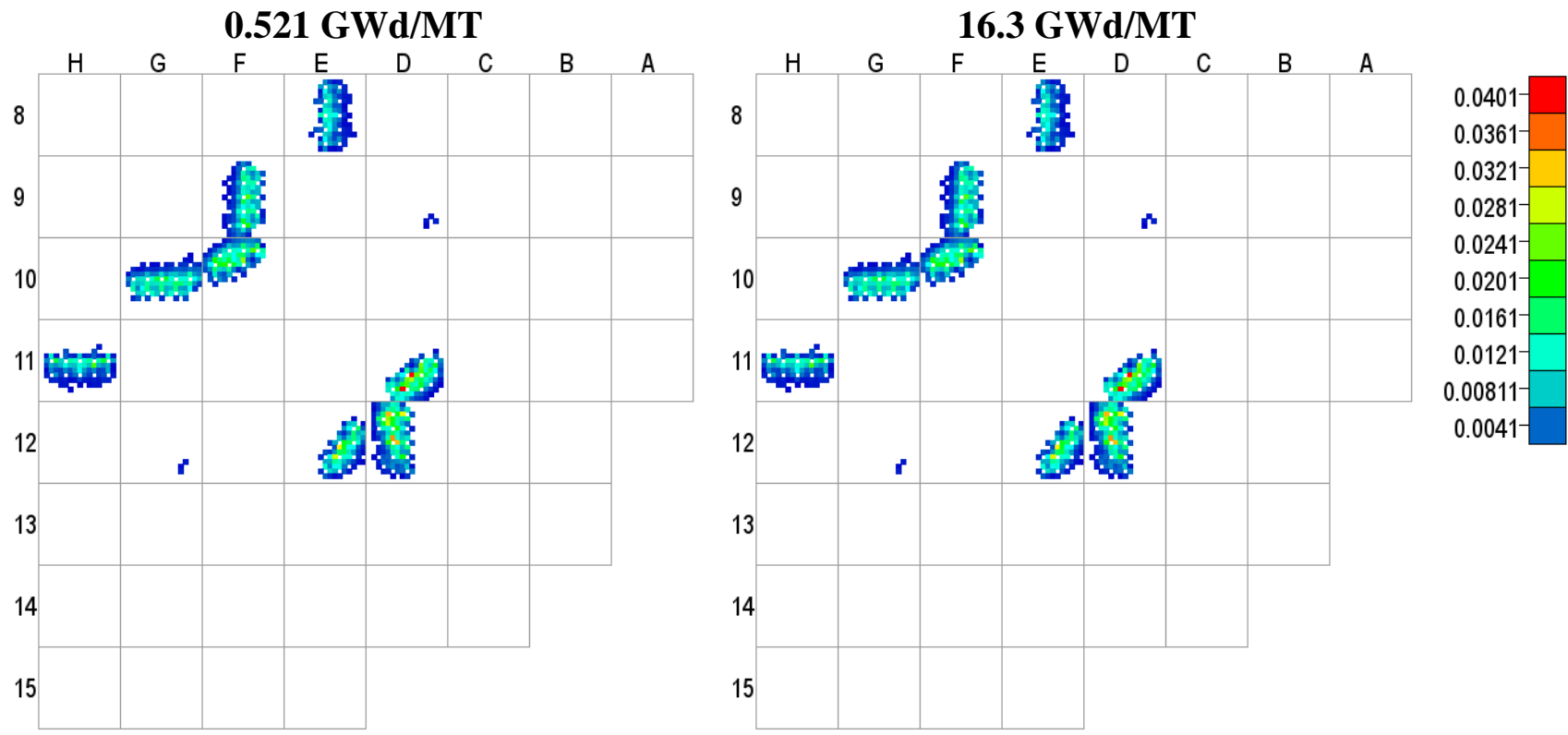

Fig. 5.3.6. Cycle 2 cumulative damage index. 


\subsection{Cycle 3}

Figures 5.4.1-5.4.5 show the results for several parameters of interest for Cycle 3. In general, similar trends are observed as in Cycle 2. However, the higher enriched $(4.401 \%)$ fresh assemblies dominate the maximum centerline fuel temperature distribution early in the cycle.
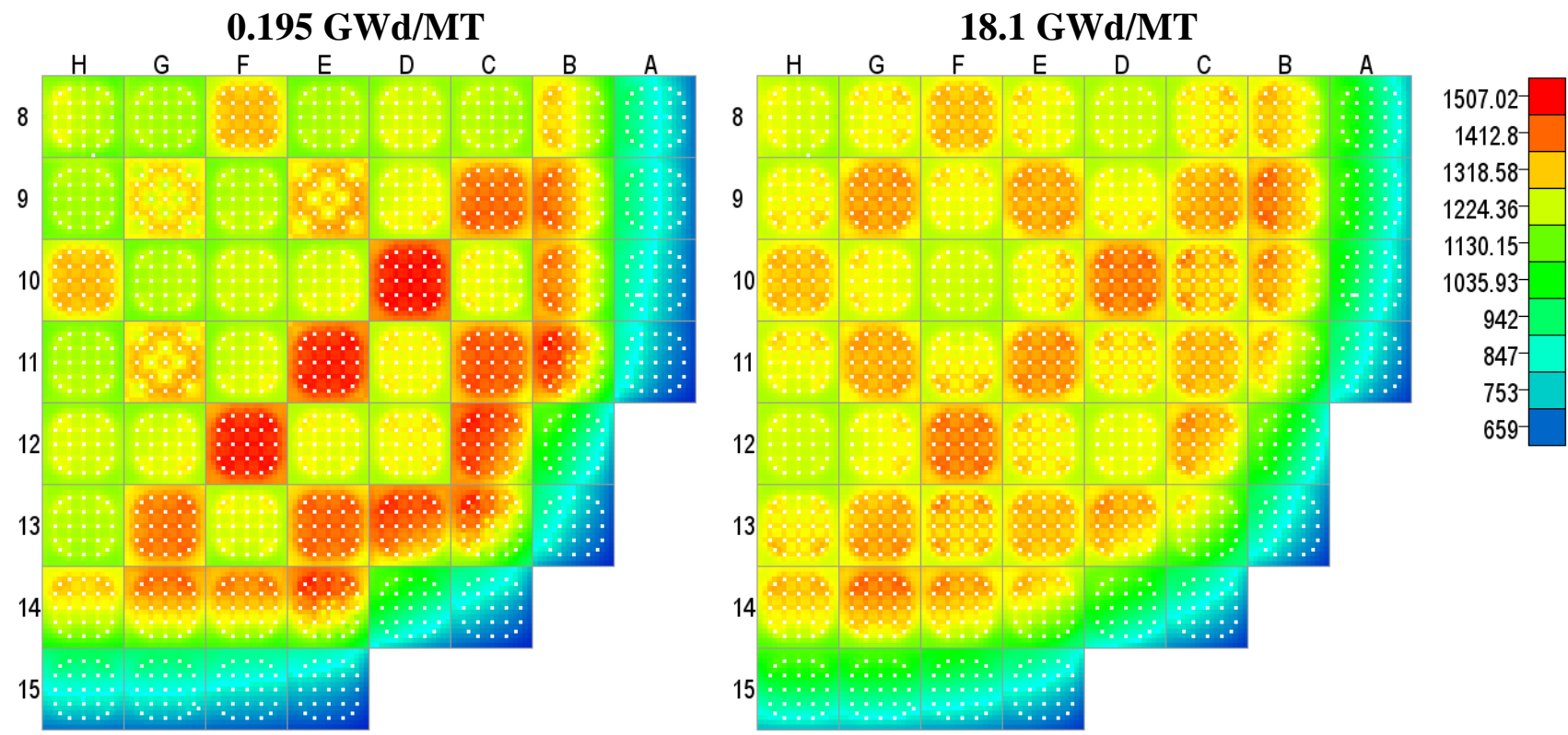

Fig. 5.4.1. Cycle 3 maximum centerline fuel temperature $(K)$.
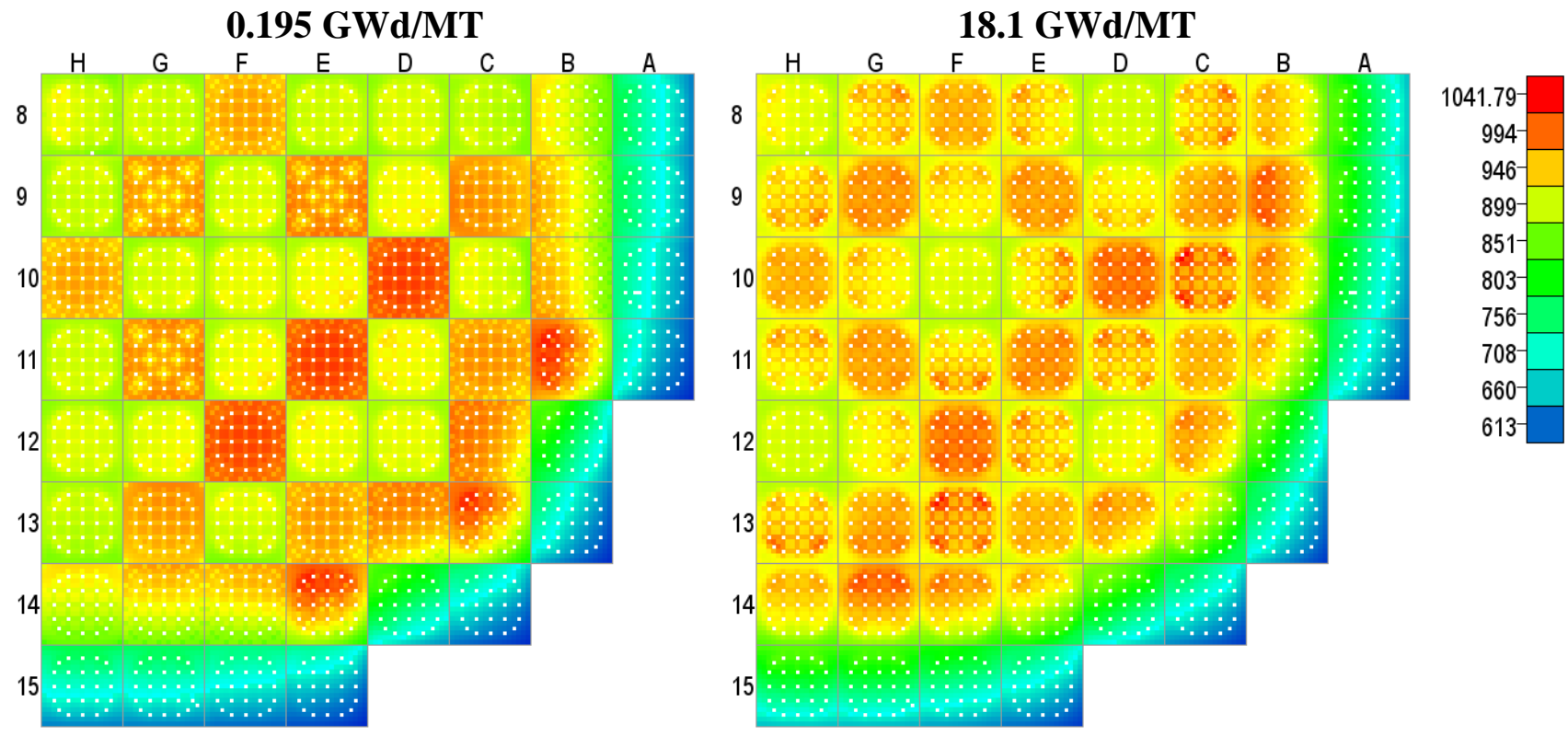

Fig. 5.4.2. Cycle 3 average fuel temperature (K). 


\subsection{GWd/MT}

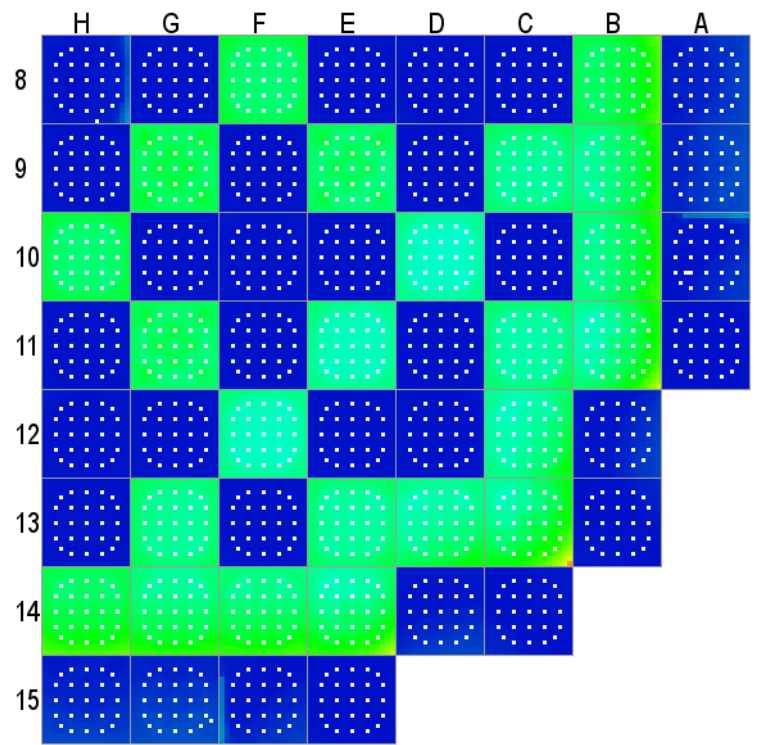

\subsection{GWd/MT}

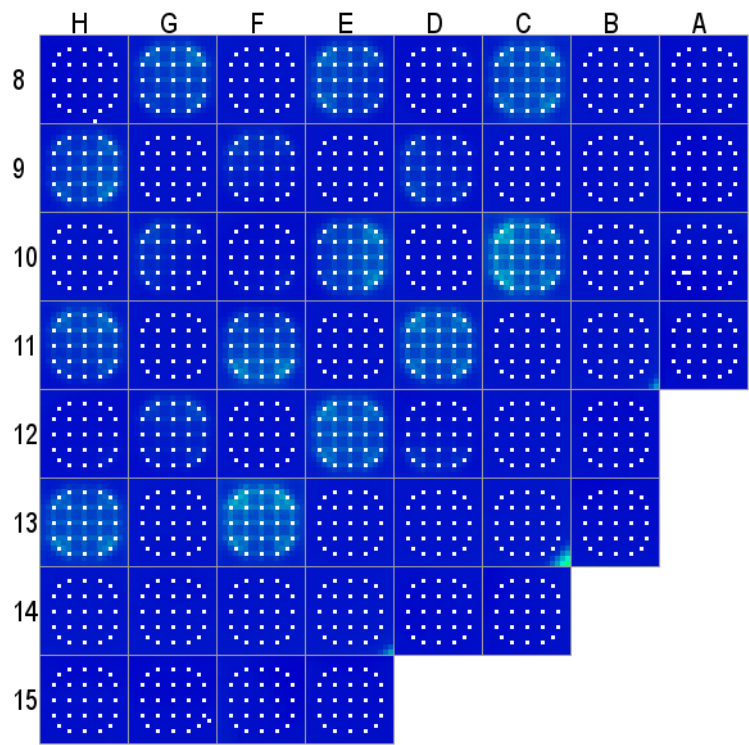

$7.65 \mathrm{e}-05$ $6.86 \mathrm{e}-05$ $6.08 \mathrm{e}-05$

$5.29 \mathrm{e}-05$ $4.5 \mathrm{e}-05$ $3.71 \mathrm{e}-05$ $2.92 \mathrm{e}-05$ $2.13 \mathrm{e}-05$ $1.34 \mathrm{e}-05$ $5.48 \mathrm{e}-06$

Fig. 5.4.3. Cycle 3 minimum gap thickness (m).
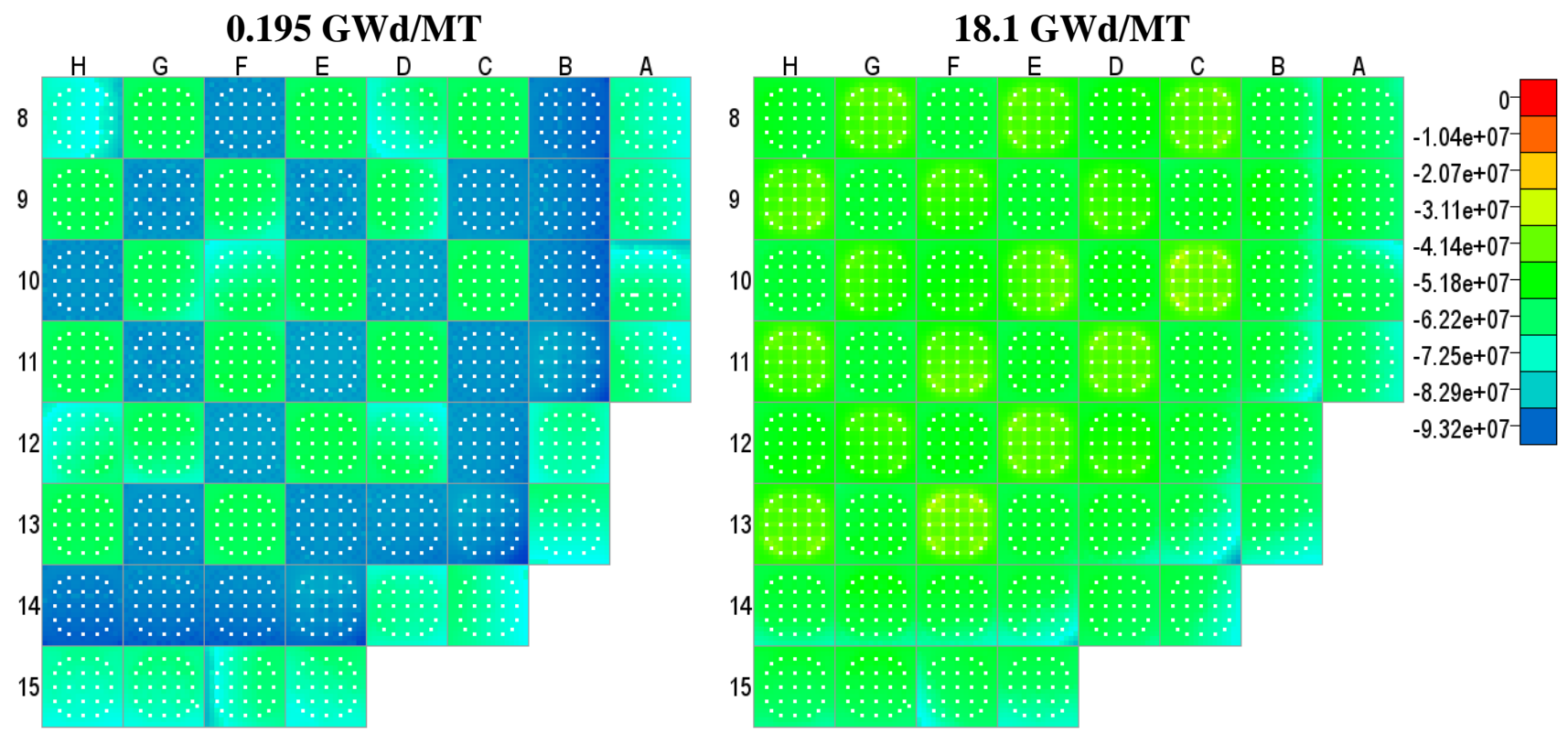

Fig. 5.4.4. Cycle 3 minimum clad hoop stress (Pa). 
It should also be noted that this core is rotationally symmetric, which accounts for some of the trends observed along the symmetry boundaries in Figure 5.4.5. Additionally, it appears that the maximum hoop stresses occur near BOC because of the ramp to power, as in Cycle 2.
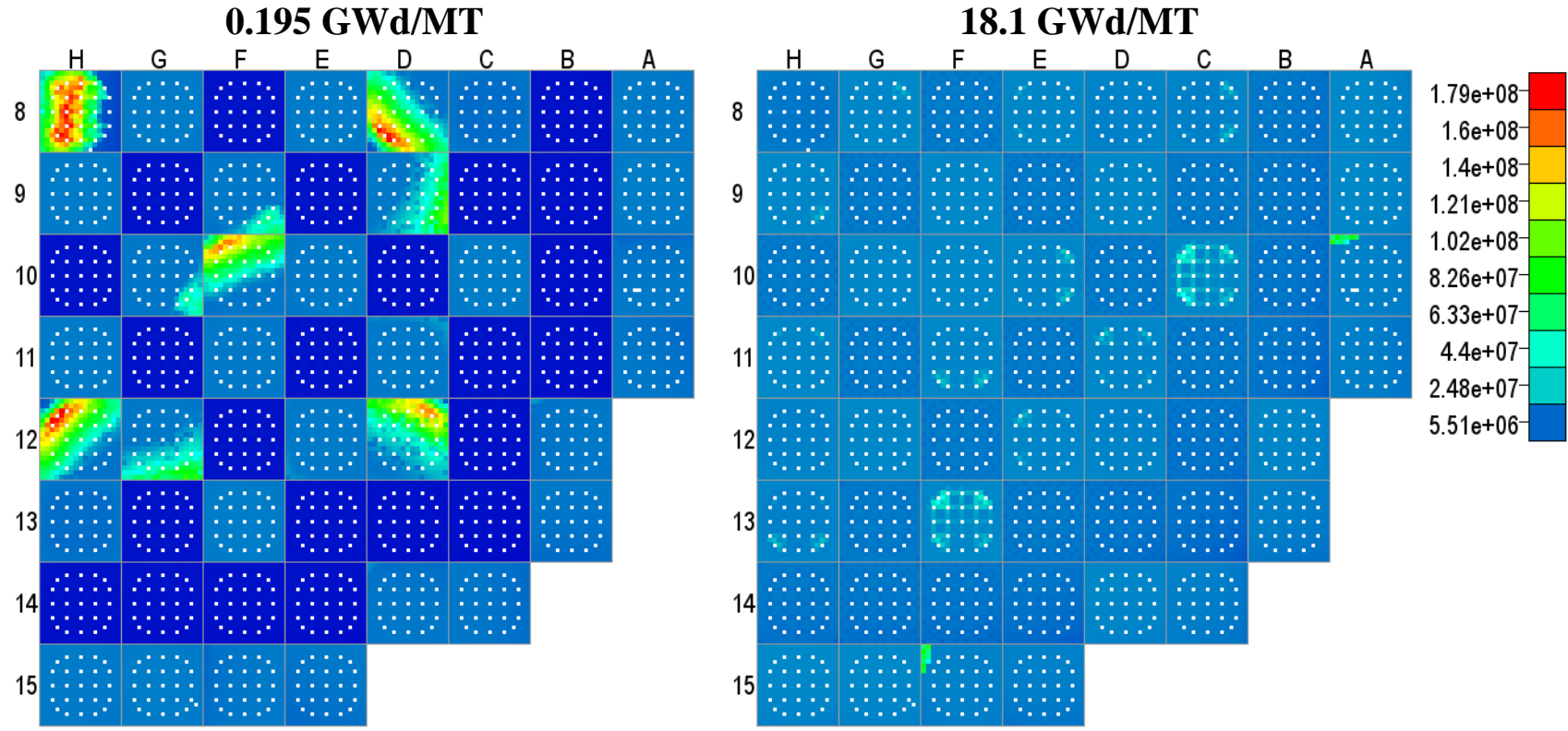

Fig. 5.4.5. Cycle 3 maximum clad hoop stress (Pa).

Similarly, the CDI corresponds well with Figure 5.4.5, but with a slightly increased maximum CDI of nearly $5 \%$.
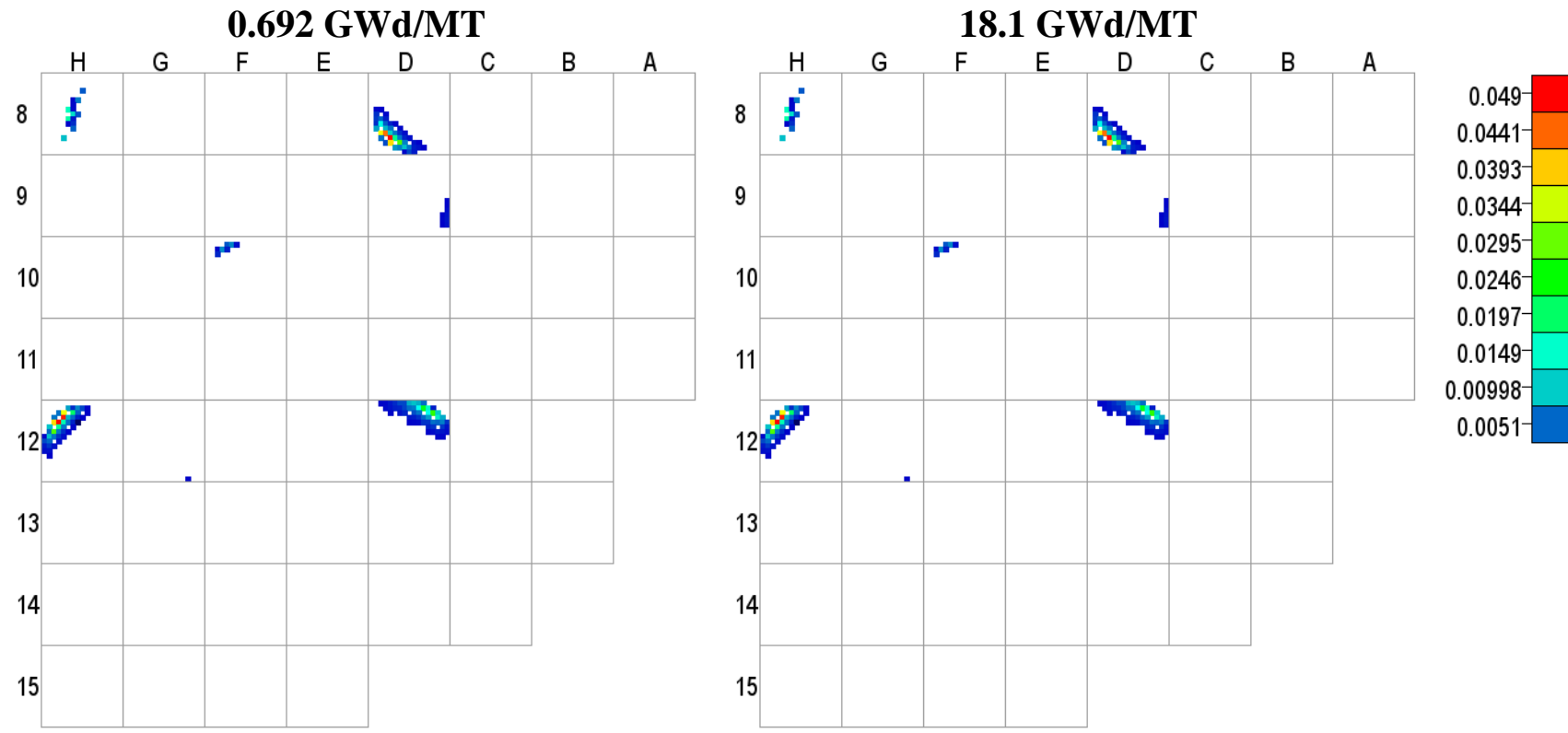

Fig. 5.4.6. Cycle 3 cumulative damage index. 


\section{CONCLUSIONS AND FUTURE WORK}

\subsection{Conclusions}

In this report, a quarter-core standalone BISON capability has been demonstrated for the first three cycles of Watts Bar Unit 1. While the current output is limited to maximum, minimum, and average quantities of interest across each fuel rod, the insights that can be obtained are still very worthwhile. This capability could be very helpful in screening for potential PCI issues as part of the challenge milestone due next year.

\subsection{Assessment of Milestone Objectives}

The objectives listed in Sect. 1 are discussed below.

\section{1) Extend XML2MOOSE to accommodate fuel shuffling in quarter symmetry cases.}

Quarter symmetry shuffling appears to be functioning appropriately. Some improvements can still be made, but it is operating as expected.

2) Develop a post-processor to collect the results from the individual BISON cases and consolidate them onto the VERAOut HDF5 file, particularly for use in VERAView.

The post-processor, which is currently termed bison-post, has been created and collects the data from the output CSVs from BISON and consolidates them onto the VERAOut HDF5 file generated by VERA-CS. The data in this file can then be easily visualized using VERAView.

3) Consider the establishment of a driver program that will simplify usage by preprocessing, running, and gathering output all in one overarching executable

Because BISON cases are running in small batches using a large number of jobs on Falcon, it is not ideal to use a single driver program to handle these jobs. If MultiApps issues are resolved completely, then a driver program can be created to handle the jobs, simplifying things for the user.

\section{4) Determine an appropriate model for simulating IFBA rods in BISON.}

The BISON team is actively developing this model, and once complete, it will require Cycles 2 and 3 to be rerun. This development should provide more insights regarding the internal pressure of the IFBA rods.

\section{5) Run several cycles of WBN1 using the VERA-CS output data available from Reference 1.}

This capability was tested using these data on the first three cycles and is currently set up to generate BISON inputs from Cycles $1-X$, depending on what is specified in the input. It may be useful to change how this works to allow users to specify that they only want results for Cycle X. This may lead to some redundancy if consecutive cycles are desired, but it could be accommodated.

6) Assess temperatures, stresses, and other important parameters of interest to PCI screening

A number of parameters of interest have been shown, including maximum centerline fuel temperature, average fuel temperate, clad hoop stresses, minimum gap thickness, and cumulative damage index. Data are also available for the clad temperatures, but they were not shown in this report. 


\section{Two additional stretch goals:}

\section{1) Compare these results to Bratton's FRAPCON results [20].}

No comparisons for full WBN1 simulations have been completed yet. However, as part of ongoing work to improve the fuel temperature tables, comparisons between FRAPCON and BISON have been completed as a function of linear heat rate and burnup, generally with good agreement.

\section{2) Perform a mesh sensitivity study to assess the appropriate refinement level.}

A simple mesh sensitivity study was performed to justify the use of a coarse quad configuration. In general, anything finer than the coarse linear parameters is sufficient for temperature, but the power discrepancy can be substantial, even in some of the slightly more refined cases. No study of time sensitivity was performed. BISON is using an adaptive time-stepping procedure that attempts to use the largest time step while still maintaining convergence (based on several parameters). Therefore, conducting a study to restrict the maximum timestep allowed would be useful.

\subsection{Running on Leadership Class Clusters}

Running a large number of BISON cases using the MultiApps capability has continued to encounter a number of errors that require some time to investigate. Additionally, the BISON team is actively working to resolve a bug with MultiApps in which all of the CSV output files are not generated appropriately. MultiApps may be necessary to use this capability on machines such as Titan and Eos, where the number of active jobs and jobs queued is considerably more restricted. For now, the current approach of running a number of small batches on Falcon has been sufficient to generate results, but it is clearly not ideal.

\subsection{Future Work}

\subsubsection{Accounting for More Fuel Rod Types}

The current BISON template is limited to use for standard $\mathrm{UO}_{2}$ fuel pins. Additional work should be performed to generalize the input for other types of rods, such those with IFBA-coated fuel, and gadolinia or erbia burnable absorber rods.

\subsubsection{Biased Meshing}

An internal INL report on BISON mesh sensitivity showed that biased (non-uniform) meshes can significantly reduce the power discrepancy between the input and calculated powers. This is something the BISON team is working on, and it will be incorporated into this capability once it is completed.

\subsubsection{Assess Effect of ZIRLO vs. Zircaloy-4}

All results here used Zircaloy-4 cladding material properties instead of a proprietary ZIRLO model, which is currently unavailable. If possible, it would be useful to obtain a ZIRLO model for comparison purposes.

\subsubsection{Oxide Layer Growth}

Oxide layer growth is an important phenomenon currently only available in the CoolantChannel model of BISON. This is actively being extended to other models, such as the one used in this work that uses a Dirichlet boundary condition on the coolant temperature.

\subsubsection{Processing More Detailed Output via EXODUS}

Accessing the more resolved data on the EXODUS output files will be essential to gaining a better understanding of the rod conditions. The CSV output data are adequate to gain some insight, but having axially dependent data will provide significantly more information. 


\subsubsection{Extending Analysis to Include WBN1 Cycles 6 and 7}

It was recently suggested that WBN1 Cycles 6 and 7 be tested with this capability to assess the potential for PCI failures. Cycle 7 is known to have substantial crud-induced power shift (CIPS) and will be an interesting test for this capability. It may be useful to allow the users to generate only inputs for Cycle $\mathrm{X}$ if desired. This may provide sufficient motivation to do that. Additionally, new fuel temperature tables have been obtained with both BISON and FRAPCON, and work is underway to reassess the temperatures in Watts Bar Cycles 1-12. It may be advantageous to wait until these new simulations have been completed before continuing BISON analysis for later cycles.

\subsubsection{Assessing Power Ramp Procedures}

One application for future work may be to assess the impact of ramping speed on potential rod failures. There may be other computational or experimental results that would provide a useful basis for comparison. 


\section{REFERENCES}

[1] A. Godfrey et al. VERA Benchmarking Results for Watts Bar Nuclear Plant Unit 1 Cycles 112. Technical Report, CASL-U-2015-0206-000, Oak Ridge National Laboratory. http://www.casl.gov/docs/CASL-U-2015-0206-000.pdf (2015).

[2] R. P. Pawlowski, K. T. Clarno, and R. O. Montgomery. Demonstrate Integrated VERA-CS for the PCI Challenge Problem. Technical Report, CASL-I-2014-0153-000, Oak Ridge National Laboratory (2014).

[3] R. P. Pawlowski et al. "Design of a high fidelity core simulator for analysis of pellet clad interaction." In: Proceedings of the ANS [American Nuclear Society] Joint International Conference on Mathematics and Computation (M\&C 2015), Supercomputing in Nuclear Applications (SNA) and the Monte Carlo (MC) Method. Nashville, TN, USA (2015).

[4] K. T. Clarno et al. "High fidelity modeling of pellet-clad interaction using the CASL virtual environment for reactor applications." In: Proc. ANS Joint International Conference on Mathematics and Computation (M\&C 2015), Supercomputing in Nuclear Applications (SNA) and the Monte Carlo (MC) Method. Nashville, TN, USA (2015).

[5] J. A. Turner. Virtual Environment for Reactor Applications (VERA): Snapshot 3.1. Technical Report, CASL-U-2013-0164-000, Oak Ridge National Laboratory. http://www.casl.gov/docs/CASL-U-2013-0164-000.pdf (2013).

[6] MPACT Theory Manual. Technical Report, University of Michigan (2013).

[7] B. Collins et al. "Assessment of 2D/1D Capability in MPACT," Proc. PHYSOR 2014, Kyoto, Japan, September 28-October 3 (2014).

[8] S. G. Stimpson, B. S. Collins, T. J. Downar. "Axial Transport Solvers for the 2D/1D Scheme in MPACT,” Proc. PHYSOR 2014, Kyoto, Japan, September 28-October 3 (2014).

[9] Oak Ridge Leadership Computing Facility. "Introducing Titan - The World's \#1 Open Science Supercomputer" (2014), http://www.olcf.ornl.gov/titan/.

[10] M. N. Avramova. CTF: A Thermal Hydraulic Sub-Channel Code for LWR Transient Analyses, Users Manual. Technical Report, Pennsylvania State University, Department of Nuclear Engineering (2009).

[11] R. O. Montgomery et al. "Peregrine: Advanced modeling of pellet-cladding interaction (pci) failure in lwrs." In: Proc. TopFuel 2012 Reactor Fuel Performance Conference. Manchester, United Kingdom (2012).

[12] R. O. Montgomery et al. "Advanced pellet-cladding interaction modeling using the US DOE CASL fuel performance code: Peregrine." In: Transactions of the American Nuclear Society Annual Meeting. Reno, Nevada (2014).

[11] D. Gaston et al. "Moose: A parallel computational framework for coupled systems of nonlinear equations.” Nuclear Engineering Design, 239: pp. 1768-1778 (2009). 
[14] R. Williamson et al. "Multidimensional multiphysics simulation of nuclear fuel behavior." Journal of Nuclear Materials, 423: pp. 149-163 (2012).

[15] S. Stimpson, et al. "Executing BISON-CASL Fuel Performance Cases Using VERA-CS Output," CASL-U-2015-0321-001, Oak Ridge National Laboratory (2015).

[16] Bratton, R. N., Jessee M. A., Wieselquist, W. A. Rod Internal Pressure Quantification and Distribution Analysis Using FRAPCON. Technical Report, ORNL/TM-2015/557, Oak Ridge National Laboratory, September 30 (2015).

[17] J. D. Hales et al. "BISON Theory Manual: The Equations Behind Nuclear Fuel Analysis," Idaho National Laboratory (2015).

[18] A. Godfrey. VERA Core Physics Benchmark Progression Problem Specifications. Technical Report, CASL-U-2012-0131-004, Oak Ridge National Laboratory. http://www.casl.gov/docs/CASL-U-2012-0131-004.pdf (2012).

[19] Montgomery, R., TVA reported core performance information for Watts Bar Unit 1 Cycle 1. Technical Report, CASL-I-2012-0101-000-b, CASL. INTERNAL ONLY (TVA NGDC L30 110630 003), June 30 (2011).

[20] Montgomery, R. Core Design Information including assembly and insert ID's, core locations, and enrichments for Watts Bar Unit 1 Cycle 1. Technical Report, CASL-P-2012-0101-000-f, CASL. PROPRIETARY (TVA NPG L30 110630 002), June 30 (2011).

[21] Hall, D. and Montgomery, R. TVA reported core performance information for Watts Bar Unit 1 Cycle 2. Technical Report, CASL-I-2012-0101-000-e, CASL. INTERNAL ONLY (TVA NGDC L36 120731 802), July 30 (2012).

[22] Hall, D. and Montgomery, R. Core Design Information including assembly and insert ID's, core locations, and enrichments for Watts Bar Unit 1 Cycle 2. Technical Report, CASL-P2012-0101-000-g, CASL. PROPRIETARY (TVA NPG L36 120731 803) July 31 (2012).

[23] Hall, J. A. and Montgomery, R. A. Transmittal of WBN-1 Operational Data to CASL Project (DOE), Cycle 3. Technical Report, CASL-I-2012-0101-000-j, CASL, December 12, 2012. INTERNAL ONLY (TVA NGDC L36 121210 802) December 12 (2012).

[24] Hall, J. A. and Montgomery, R. A. Transmittal of WBN-1 Core Design Information to CASL Project (DOE), Cycle 3. Technical Report, CASL-P-2012-0101-000-i, CASL, INTERNAL ONLY (TVA NGDC L36 121210 800) December 12 (2012).

[25] S. Novascone, R. Gardner, K. Gamble, R. Williamson, personal communication, October 2829, 2015. 


\section{APPENDIX A - EXPANDED RESULTS}

\section{A.1. Cycle 1}

\section{A.1.1. Maximum Centerline Fuel Temperature}
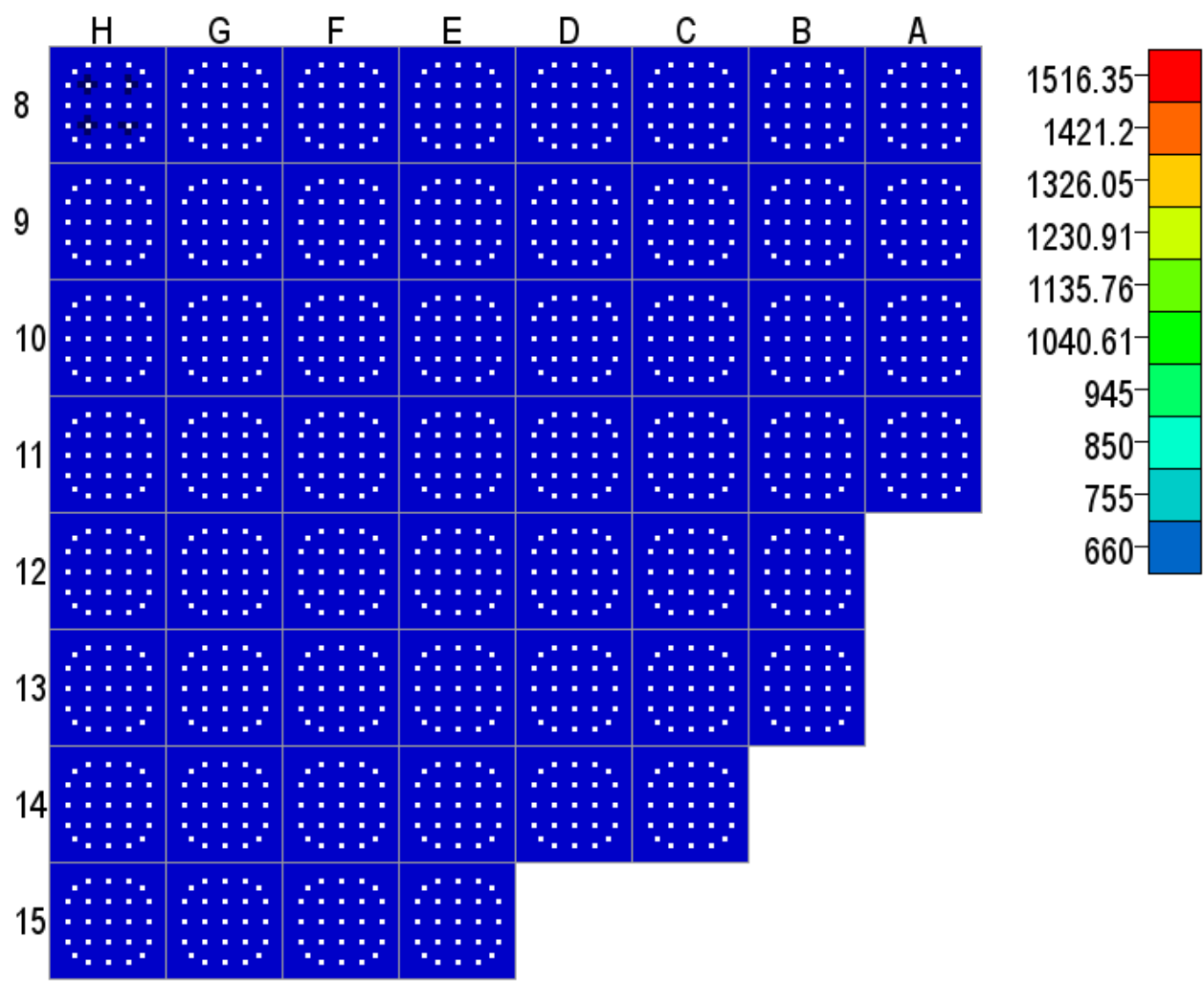

Fig. A.1.1. Cycle 1 maximum centerline fuel temperature $(\mathrm{K})-0.00 \mathrm{GWd} / \mathrm{MT}$.

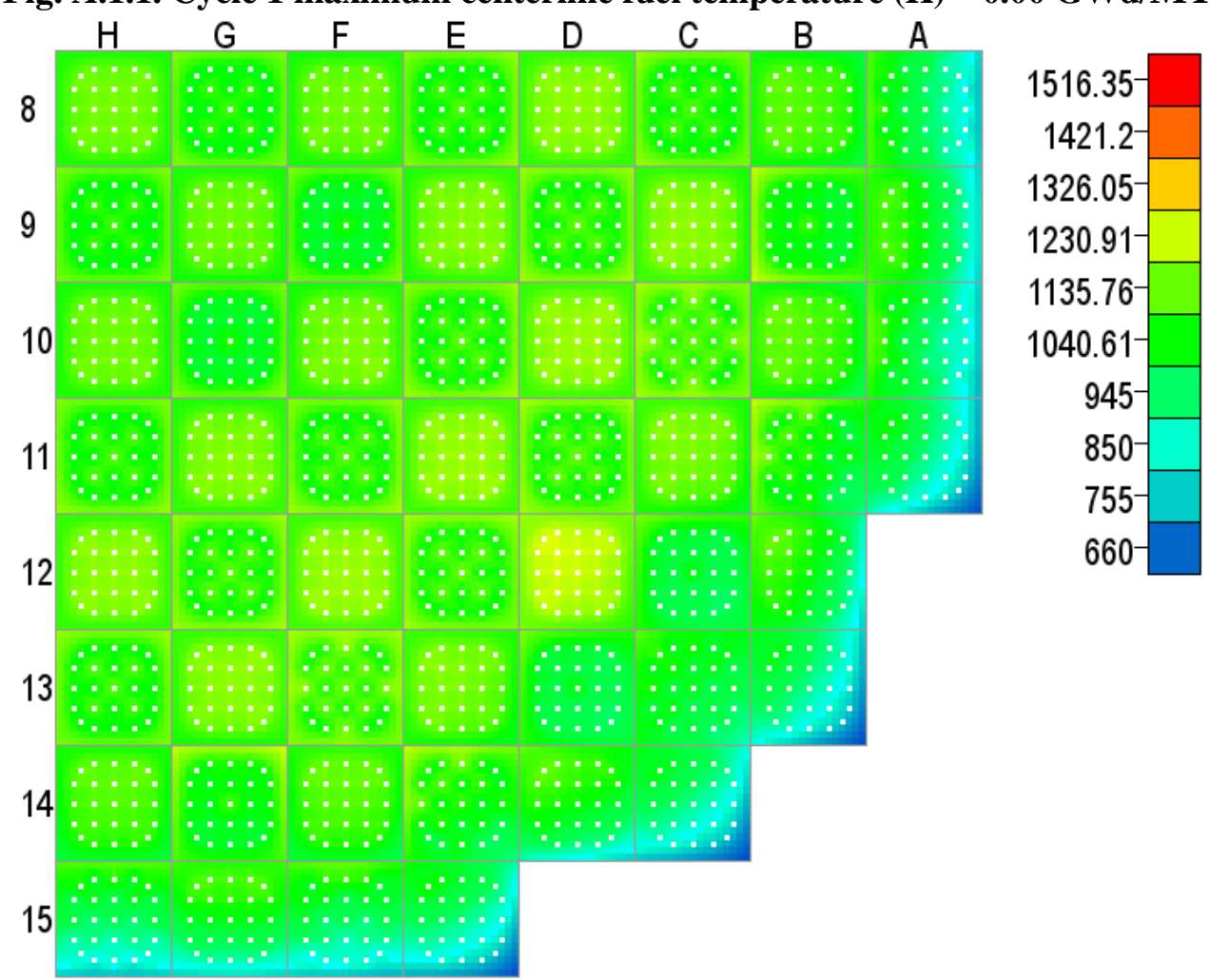

Fig. A.1.2. Cycle 1 maximum centerline fuel temperature $(\mathrm{K})-0.35 \mathrm{GWd} / \mathrm{MT}$. 


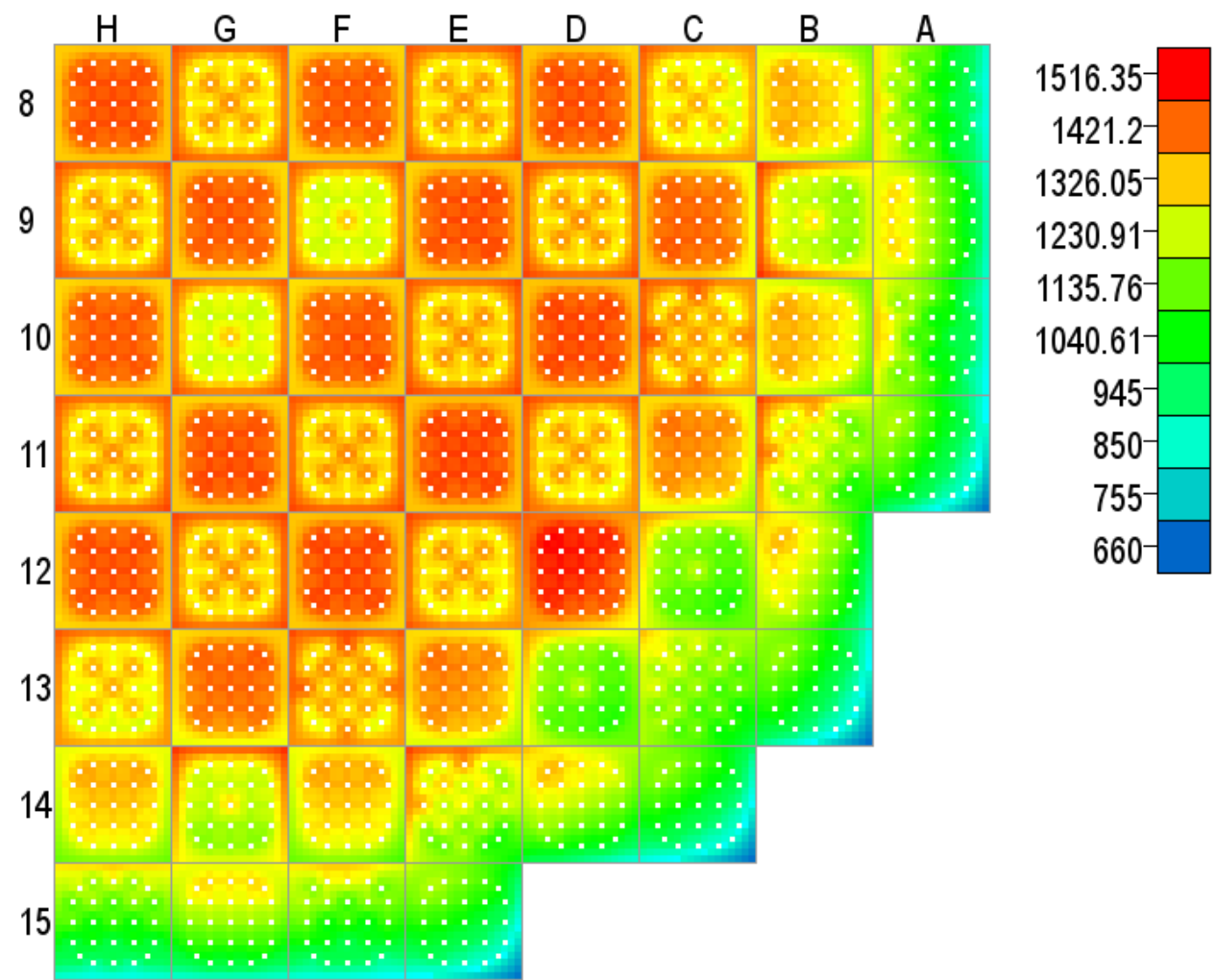

Fig. A.1.3. Cycle 1maximum centerline fuel temperature $(\mathrm{K})-1.23 \mathrm{GWd} / \mathrm{MT}$.

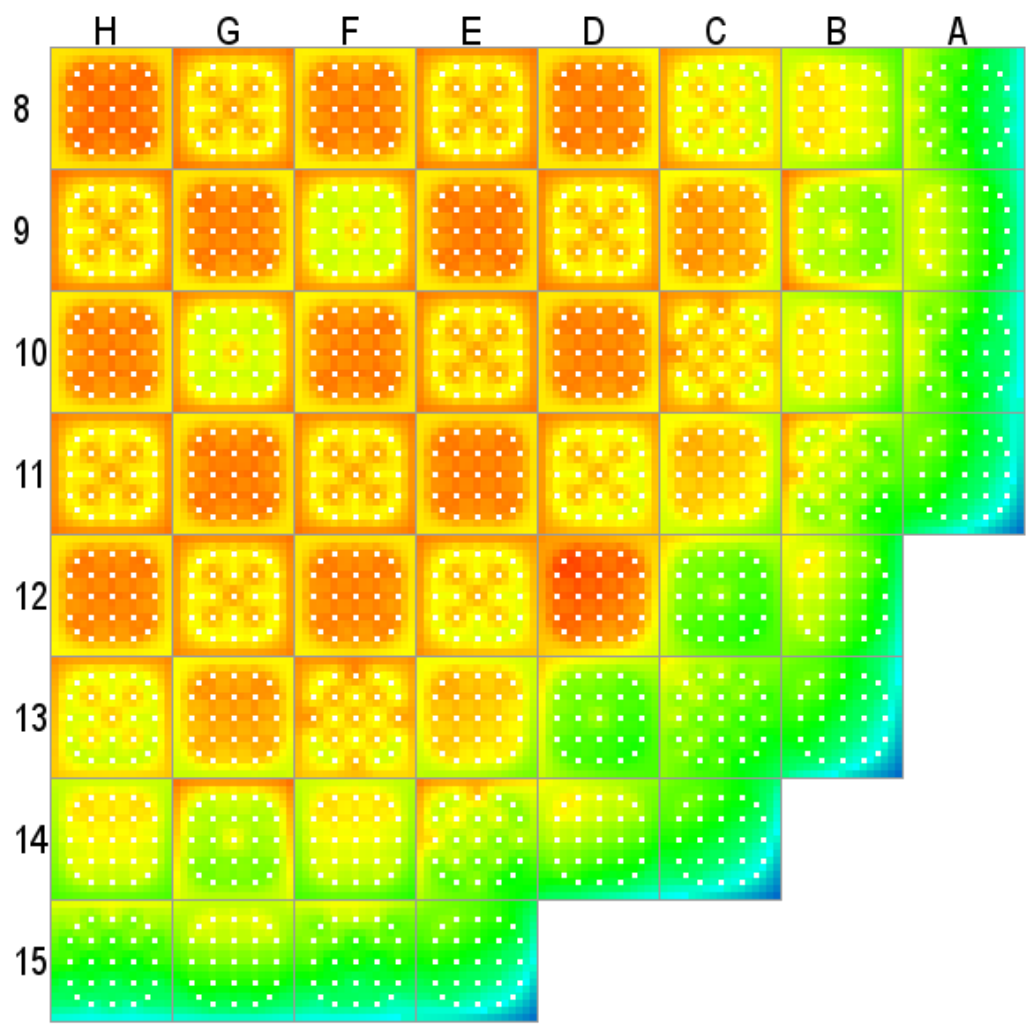

$1516.35-$
1421.2
1326.05
$1230.91-$
$1135.76-$
$1040.61-$
$945-$
$850-$
$755-$
$660-$

Fig. A.1.4. Cycle 1maximum centerline fuel temperature (K) - 1.92 GWd/MT. 


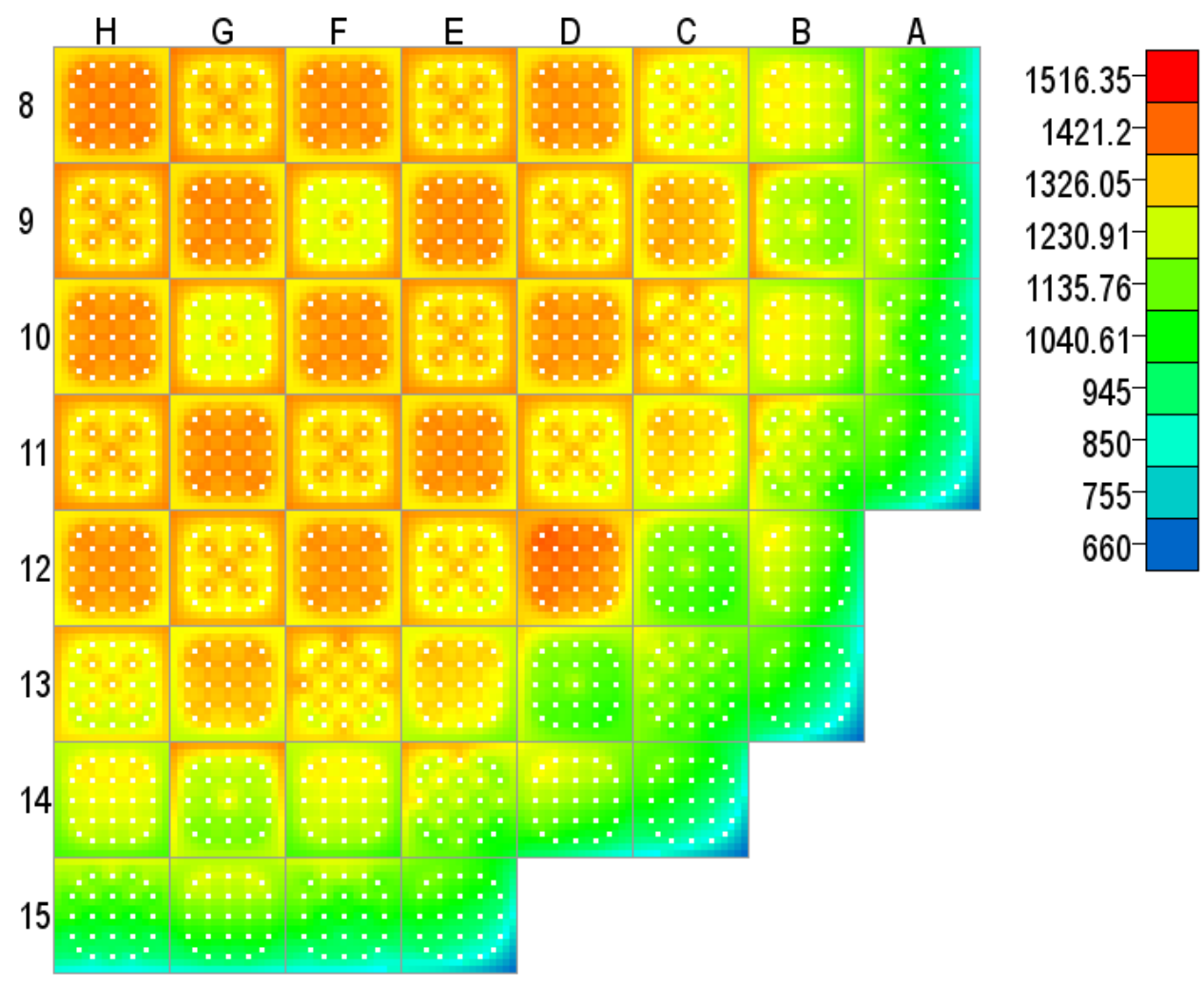

Fig. A.1.5. Cycle 1 maximum centerline fuel temperature (K) - 2.46 GWd/MT.

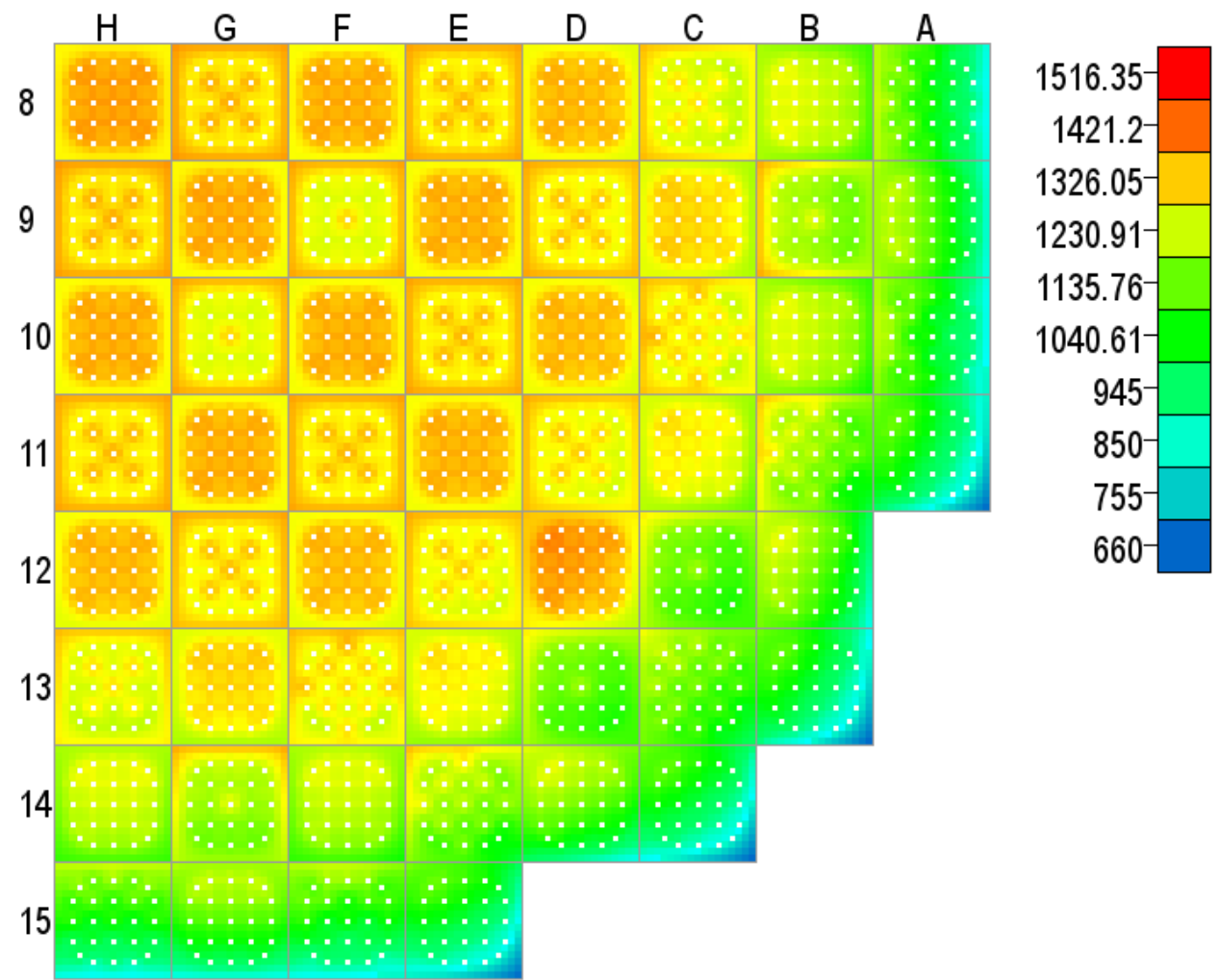

Fig. A.1.6. Cycle 1 maximum centerline fuel temperature (K) - 2.99 GWd/MT. 


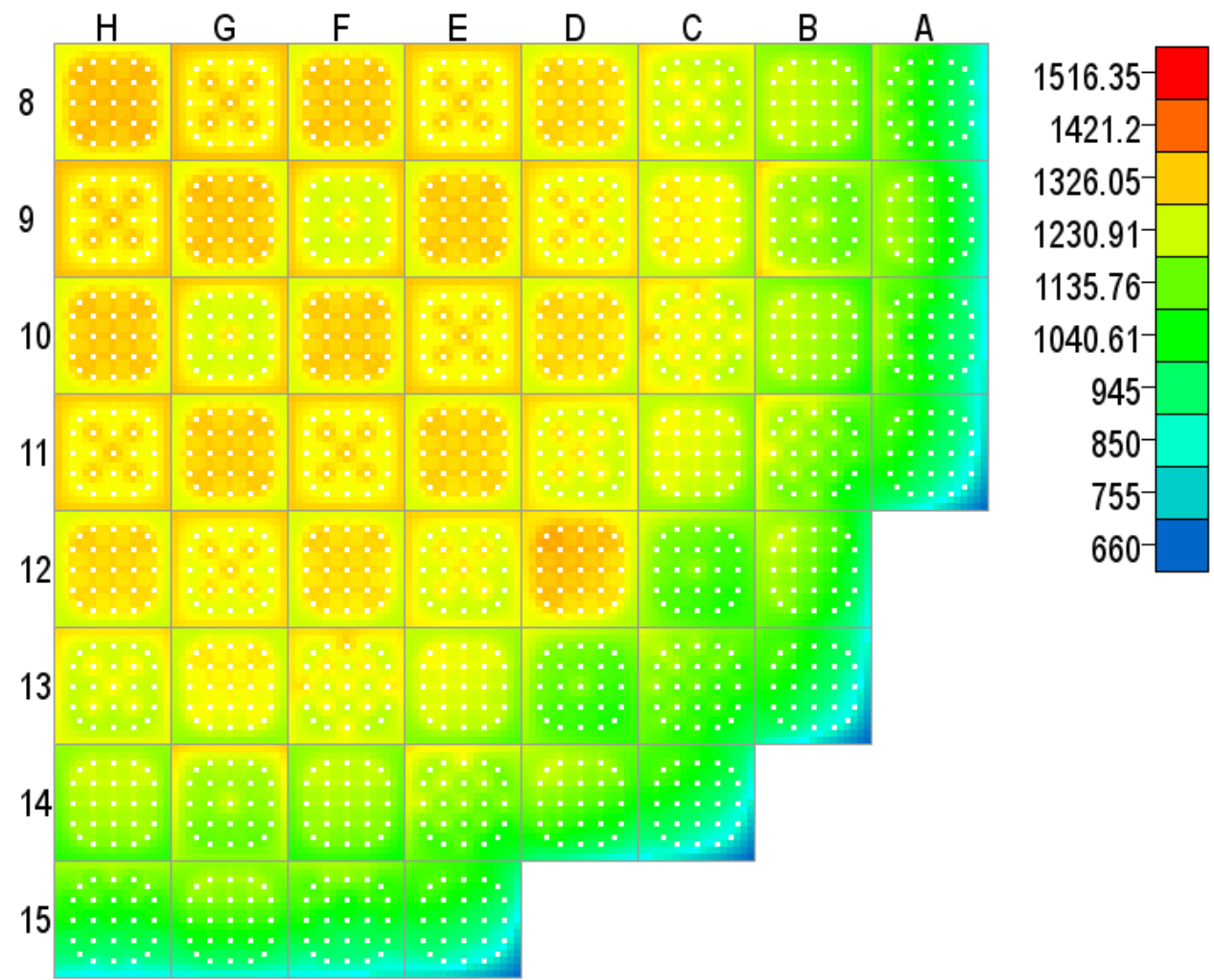

Fig. A.1.7. Cycle 1maximum centerline fuel temperature $(\mathrm{K})-3.56 \mathrm{GWd} / \mathrm{MT}$.

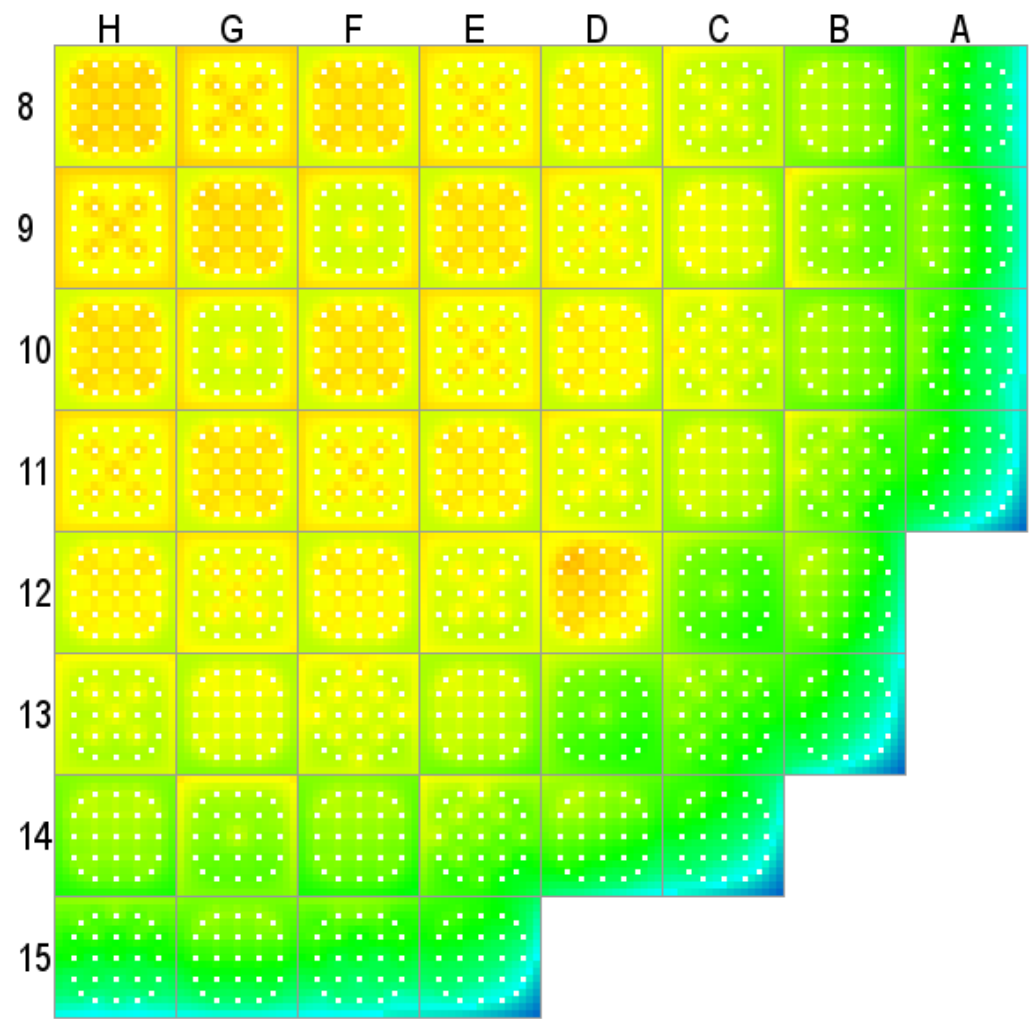

1516.35

1421.2

1326.05

1230.91

1135.76

1040.61

$945-$

850

755

660

Fig. A.1.8. Cycle 1 maximum centerline fuel temperature $(\mathrm{K})-4.07 \mathrm{GWd} / \mathrm{MT}$. 


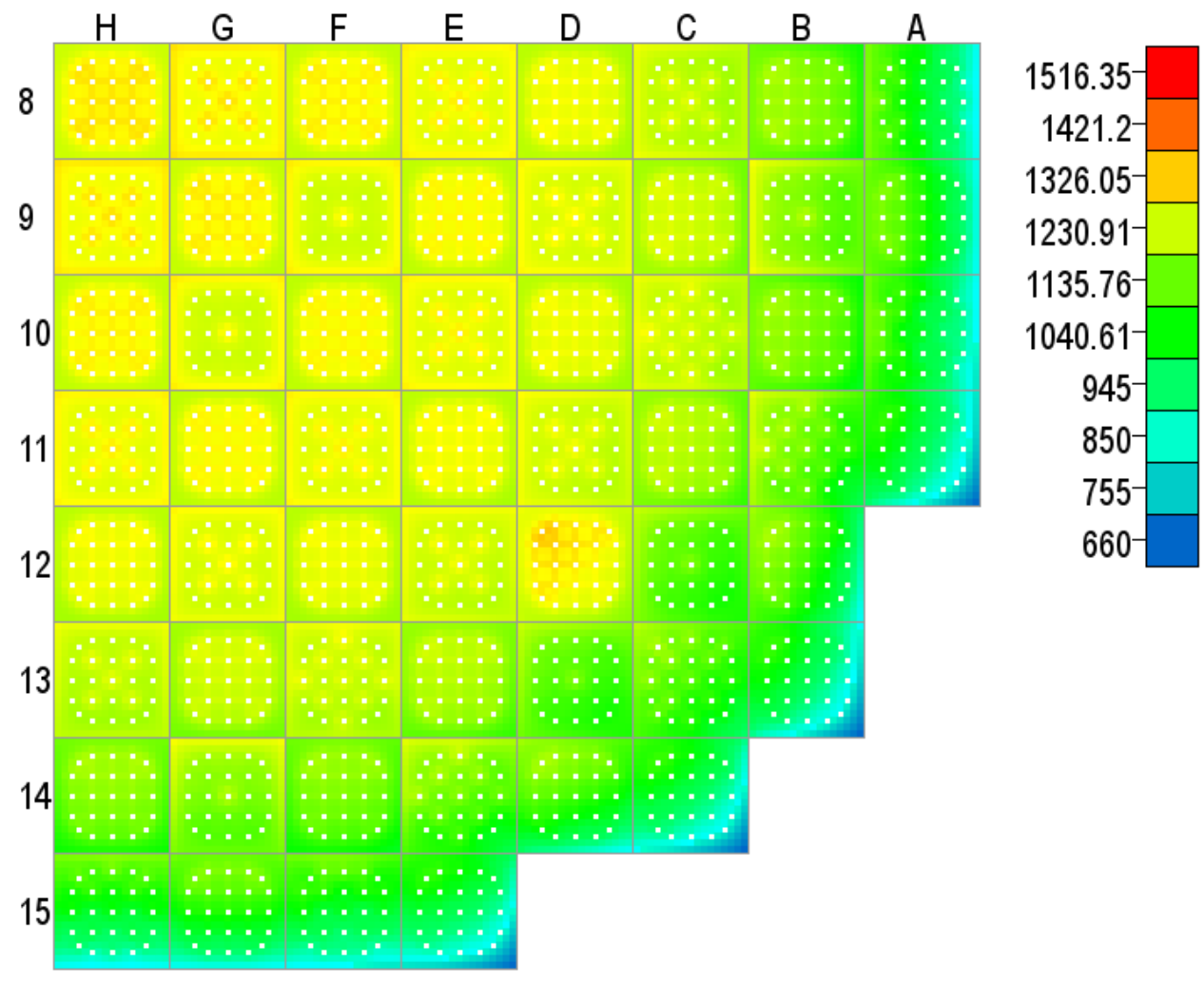

Fig. A.1.9. Cycle 1 maximum centerline fuel temperature (K) - 4.64 GWd/MT.

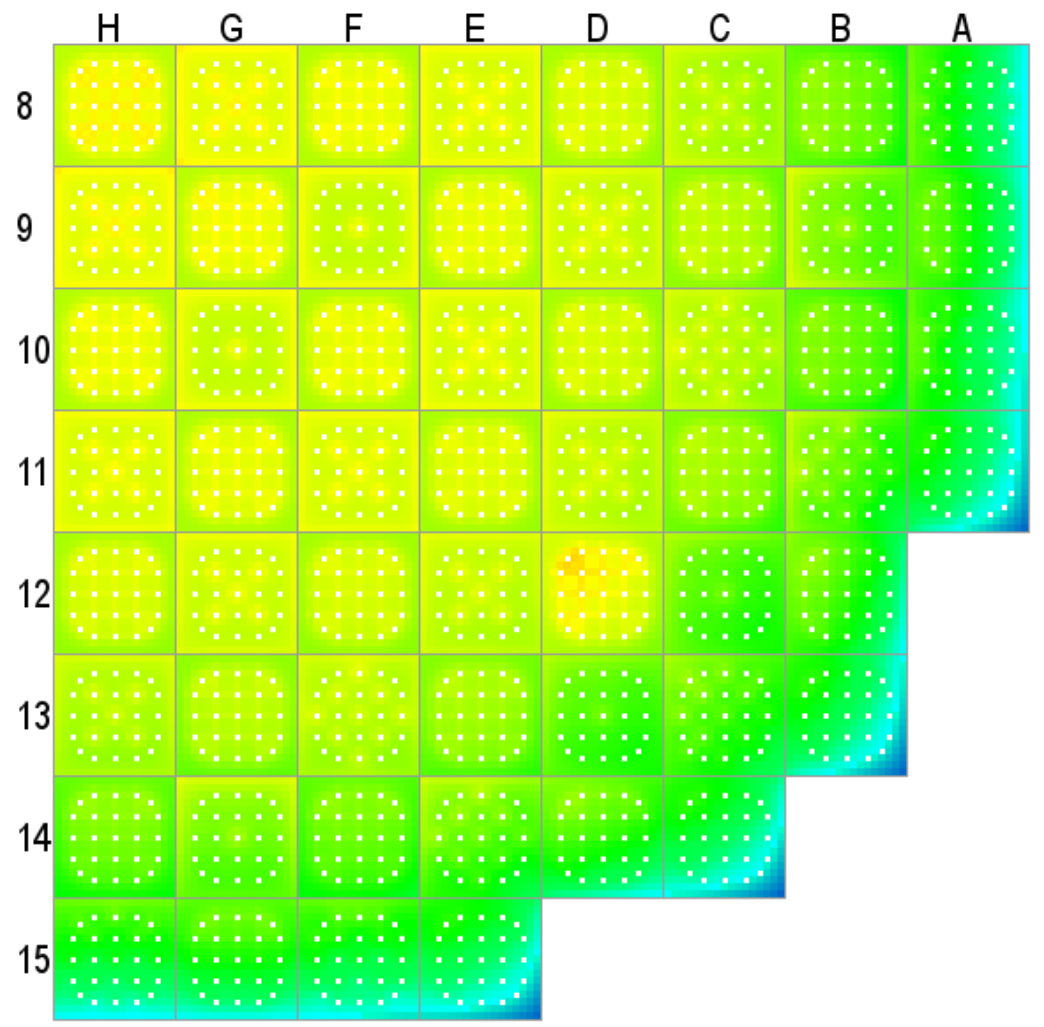

1516.35

1421.2

1326.05

1230.91

1135.76

1040.61

945

850

755

660

Fig. A.1.10. Cycle 1maximum centerline fuel temperature $(\mathrm{K})-5.14 \mathrm{GWd} / \mathrm{MT}$. 


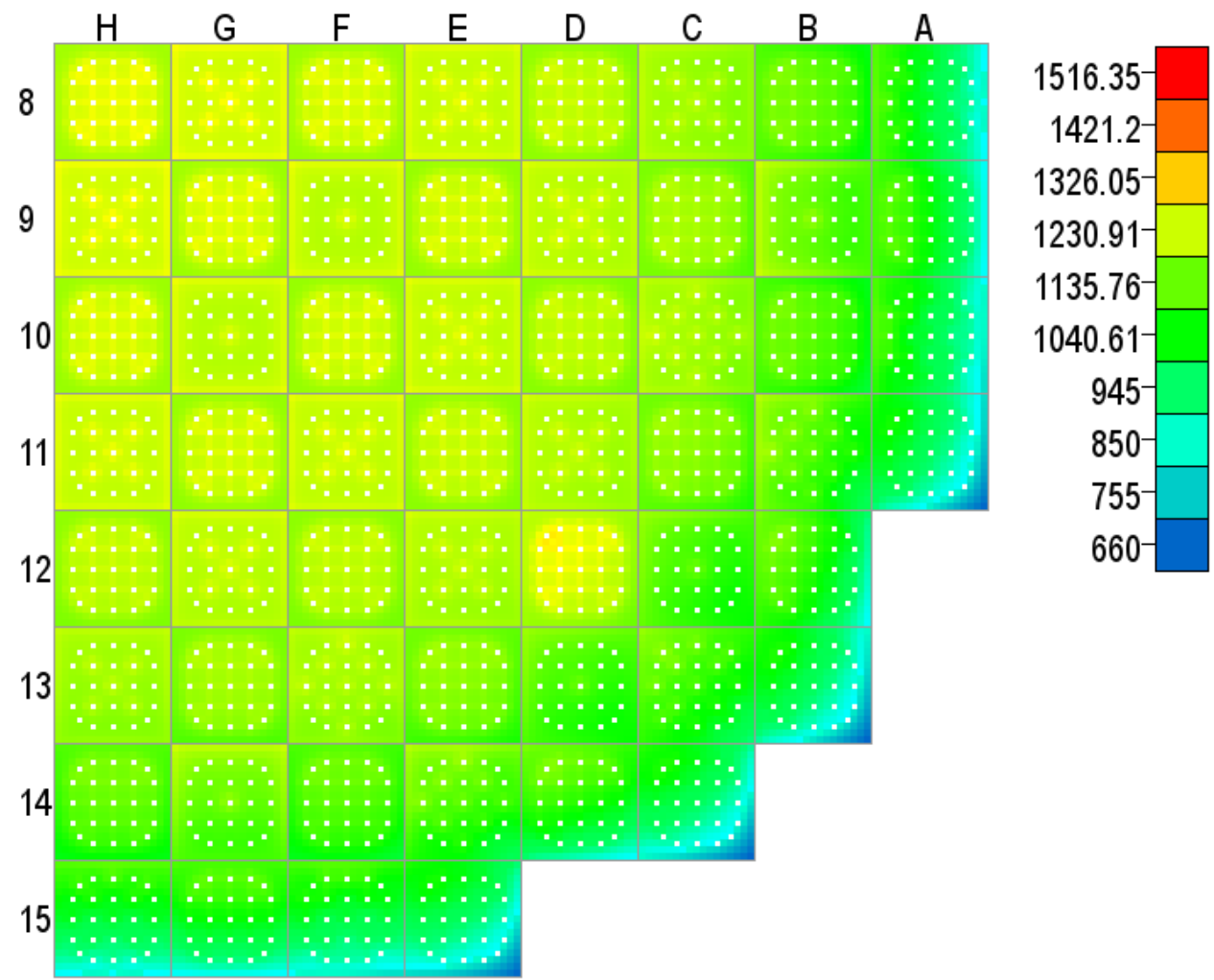

Fig. A.1.11. Cycle 1 maximum centerline fuel temperature (K) - 5.70 GWd/MT.

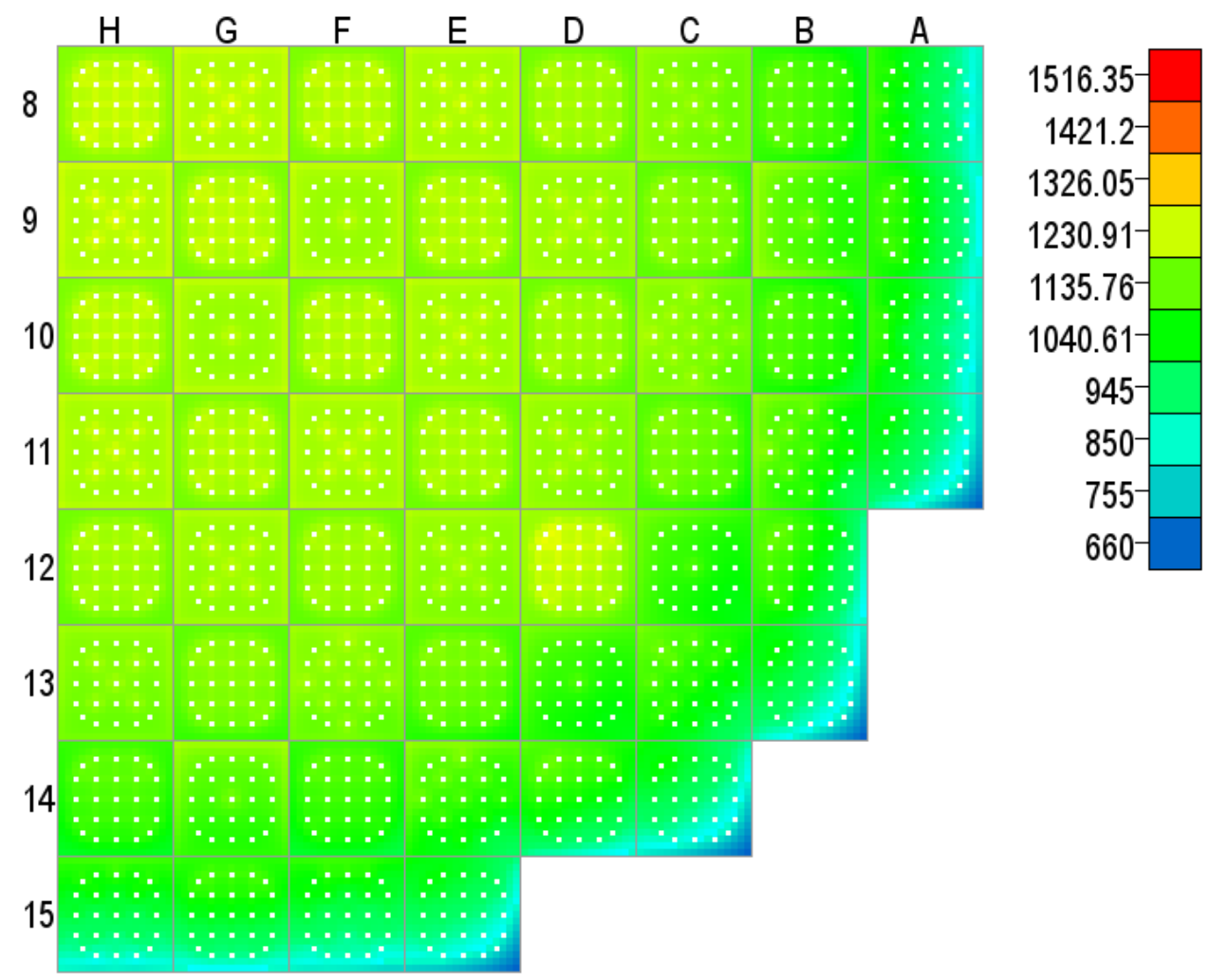

Fig. A.1.12. Cycle 1maximum centerline fuel temperature $(\mathrm{K})-6.27 \mathrm{GWd} / \mathrm{MT}$. 


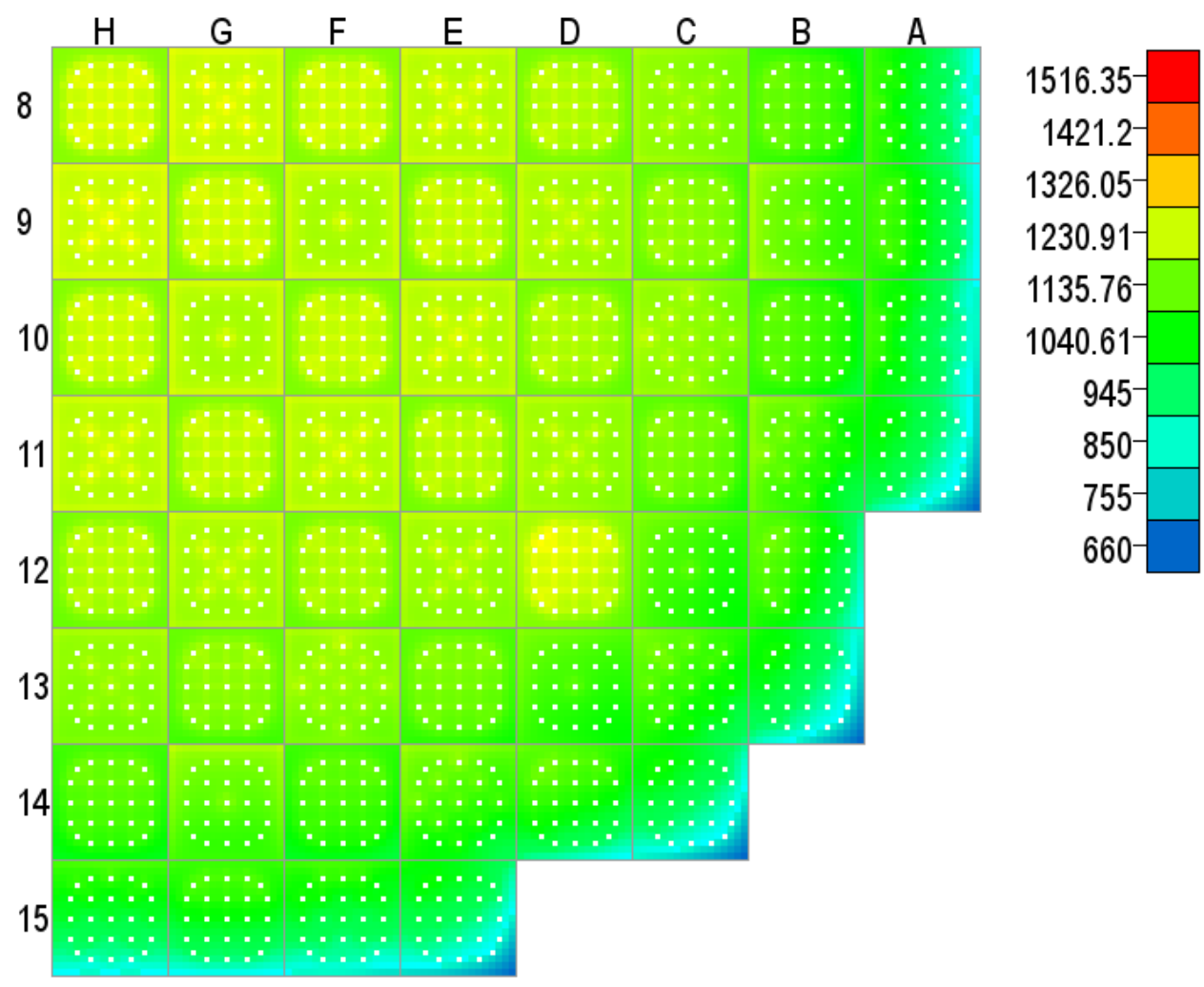

Fig. A.1.13. Cycle 1 maximum centerline fuel temperature $(\mathrm{K})-7.46 \mathrm{GWd} / \mathrm{MT}$.

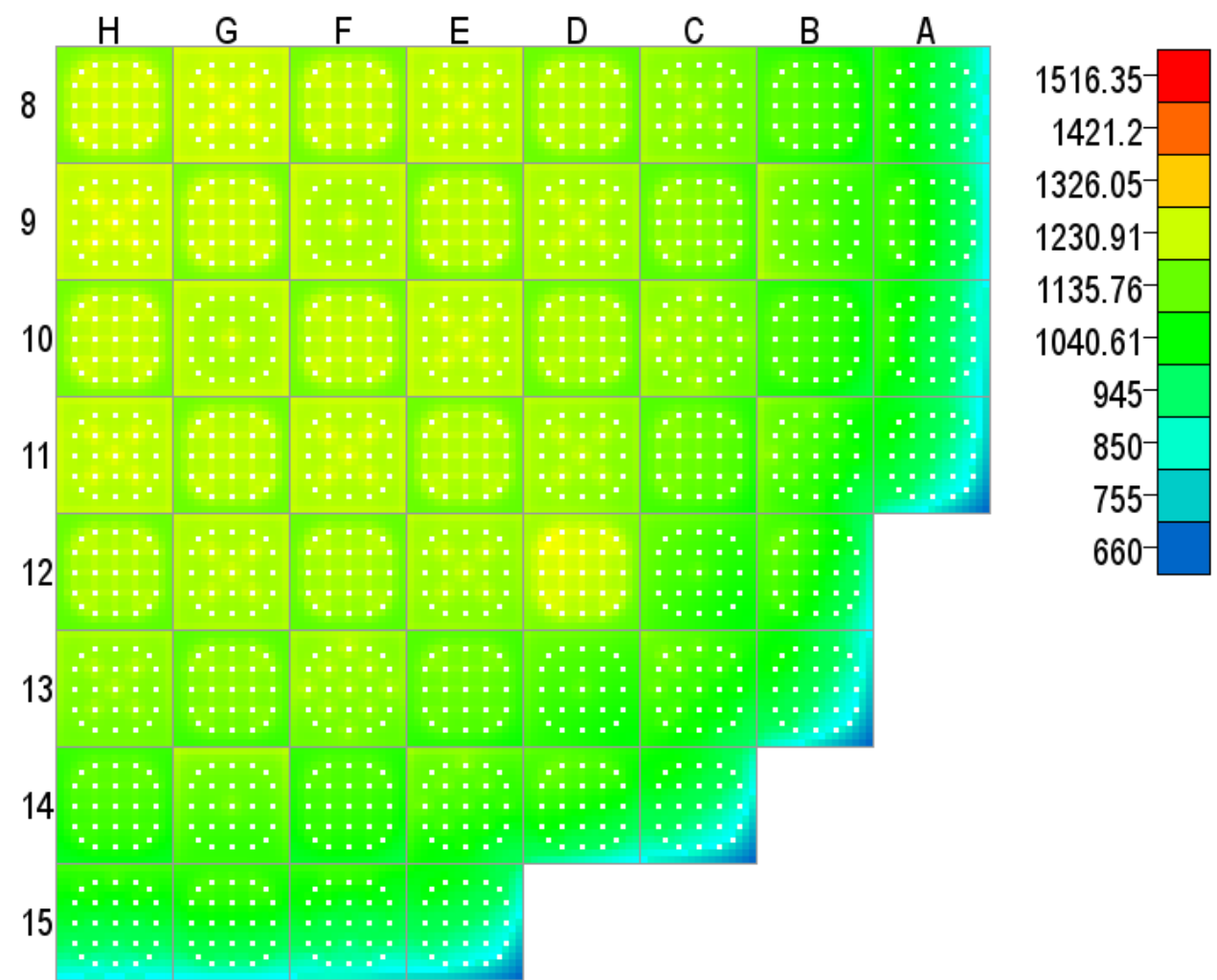

Fig. A.1.14. Cycle 1maximum centerline fuel temperature (K) - 8.49 GWd/MT. 


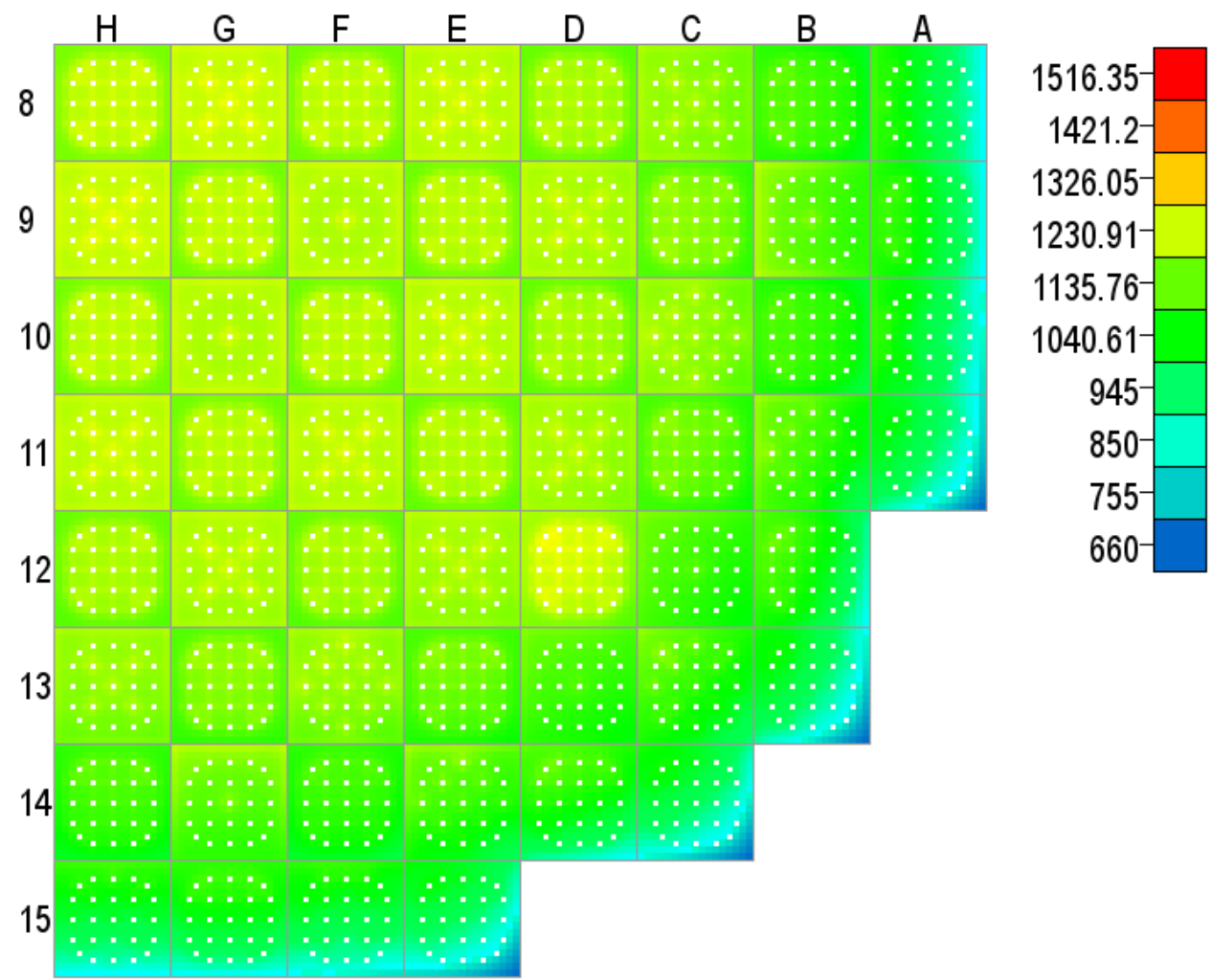

Fig. A.1.15. Cycle 1 maximum centerline fuel temperature (K) - 9.60 GWd/MT.

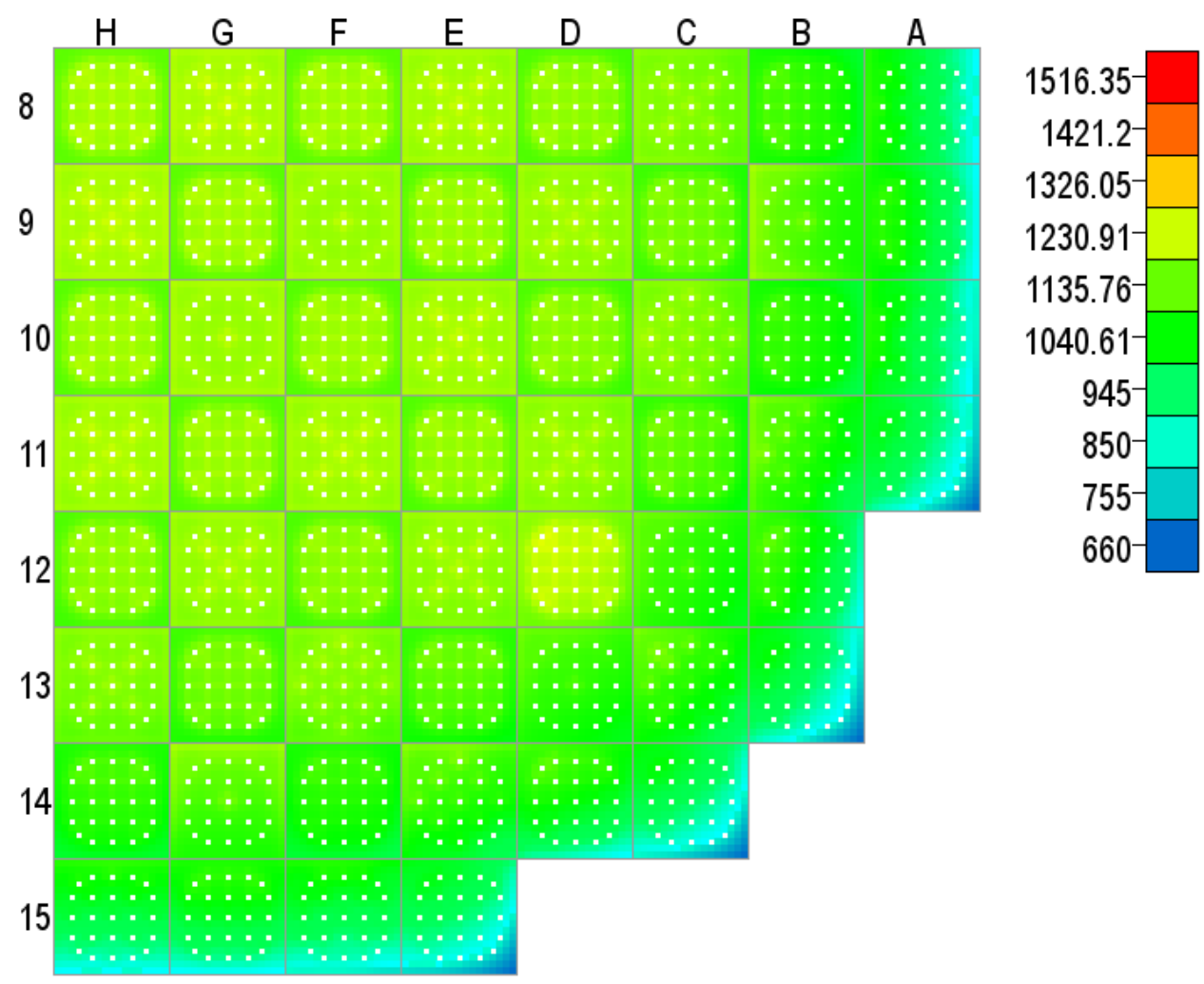

Fig. A.1.16. Cycle 1 maximum centerline fuel temperature (K) - 10.8 GWd/MT. 


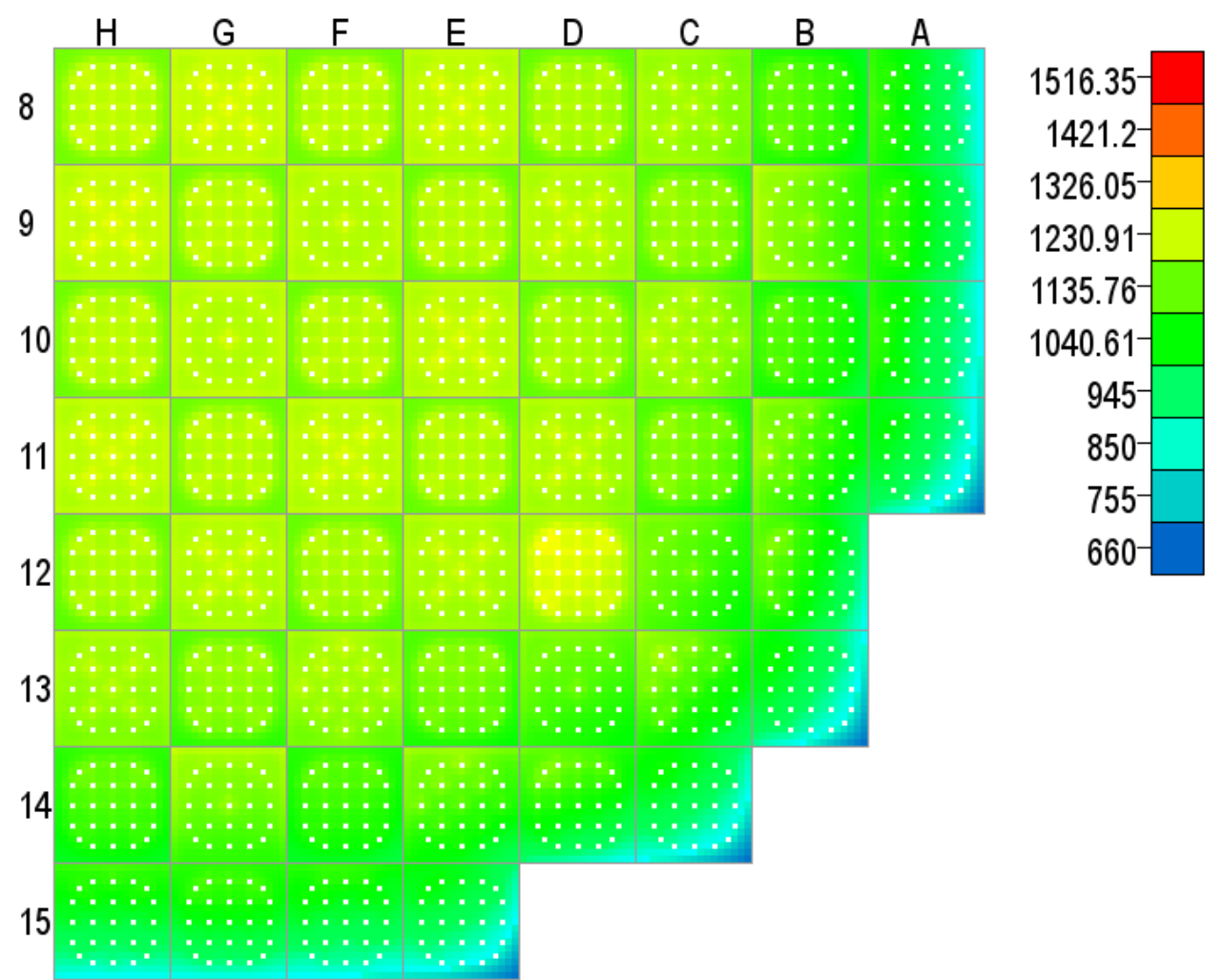

Fig. A.1.17. Cycle 1 maximum centerline fuel temperature $(\mathrm{K})-12.00 \mathrm{GWd} / \mathrm{MT}$.

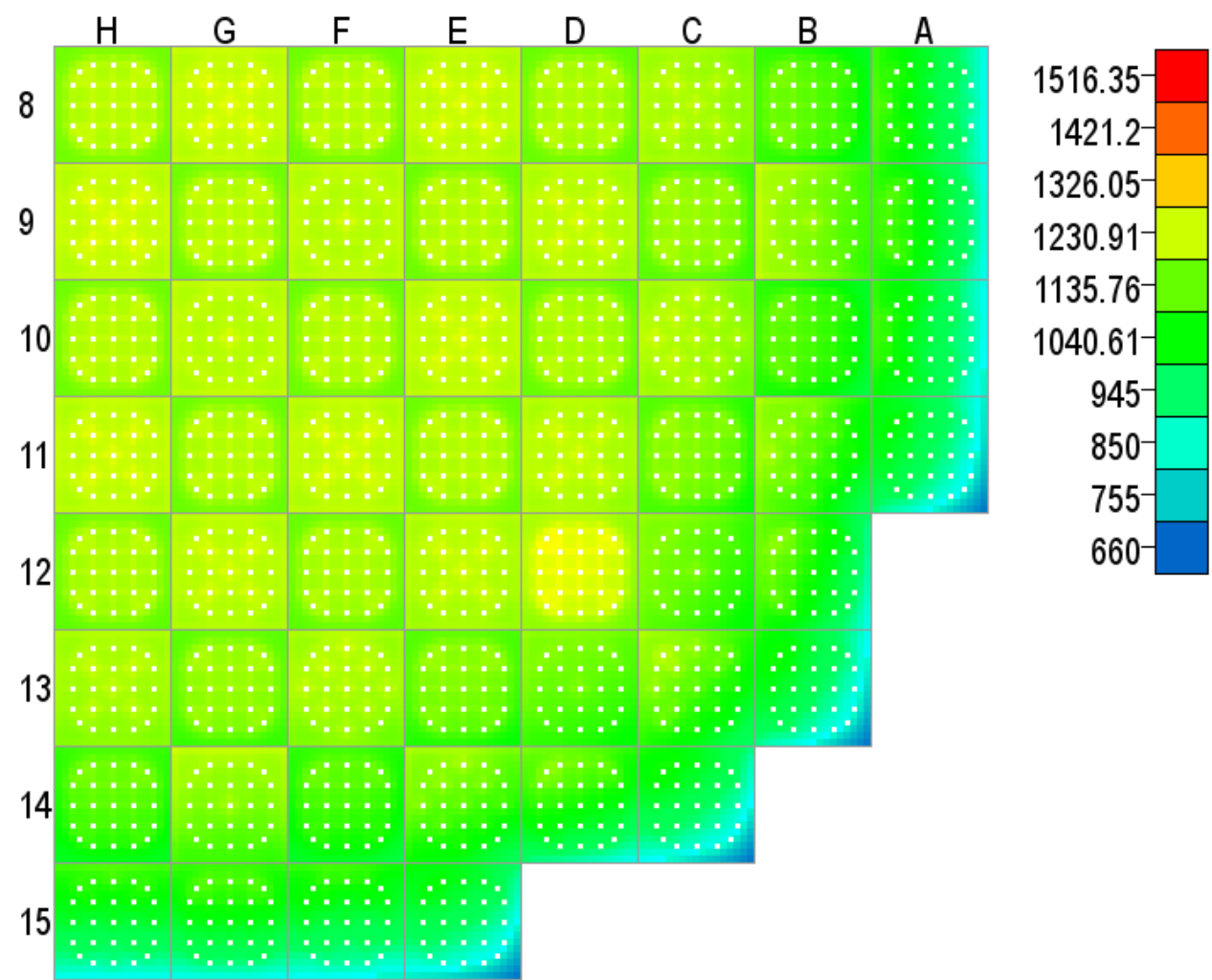

Fig. A.1.18. Cycle 1 maximum centerline fuel temperature $(\mathrm{K})-13.40 \mathrm{GWd} / \mathrm{MT}$. 


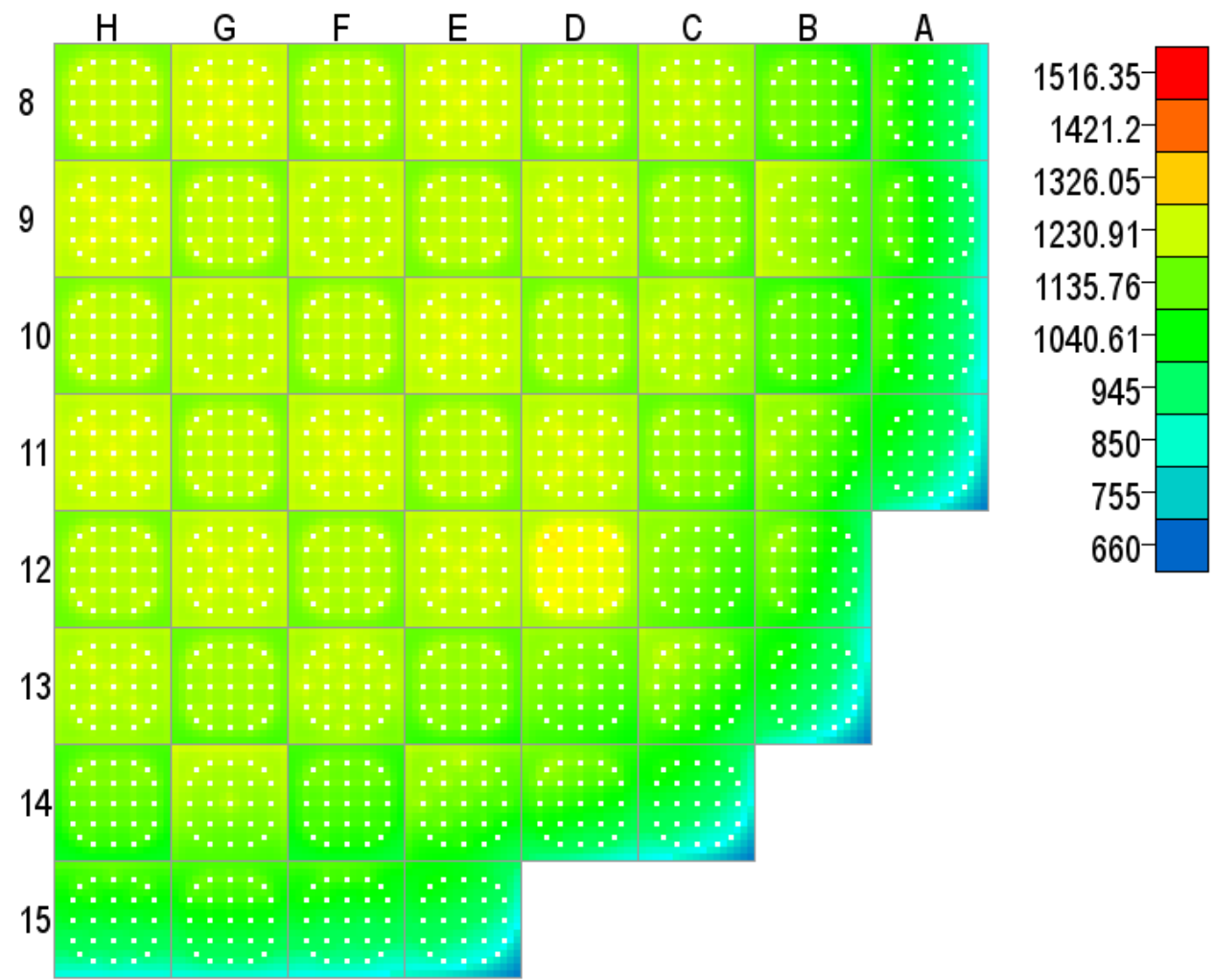

Fig. A.1.19. Cycle 1 maximum centerline fuel temperature (K) - 14.3 GWd/MT.

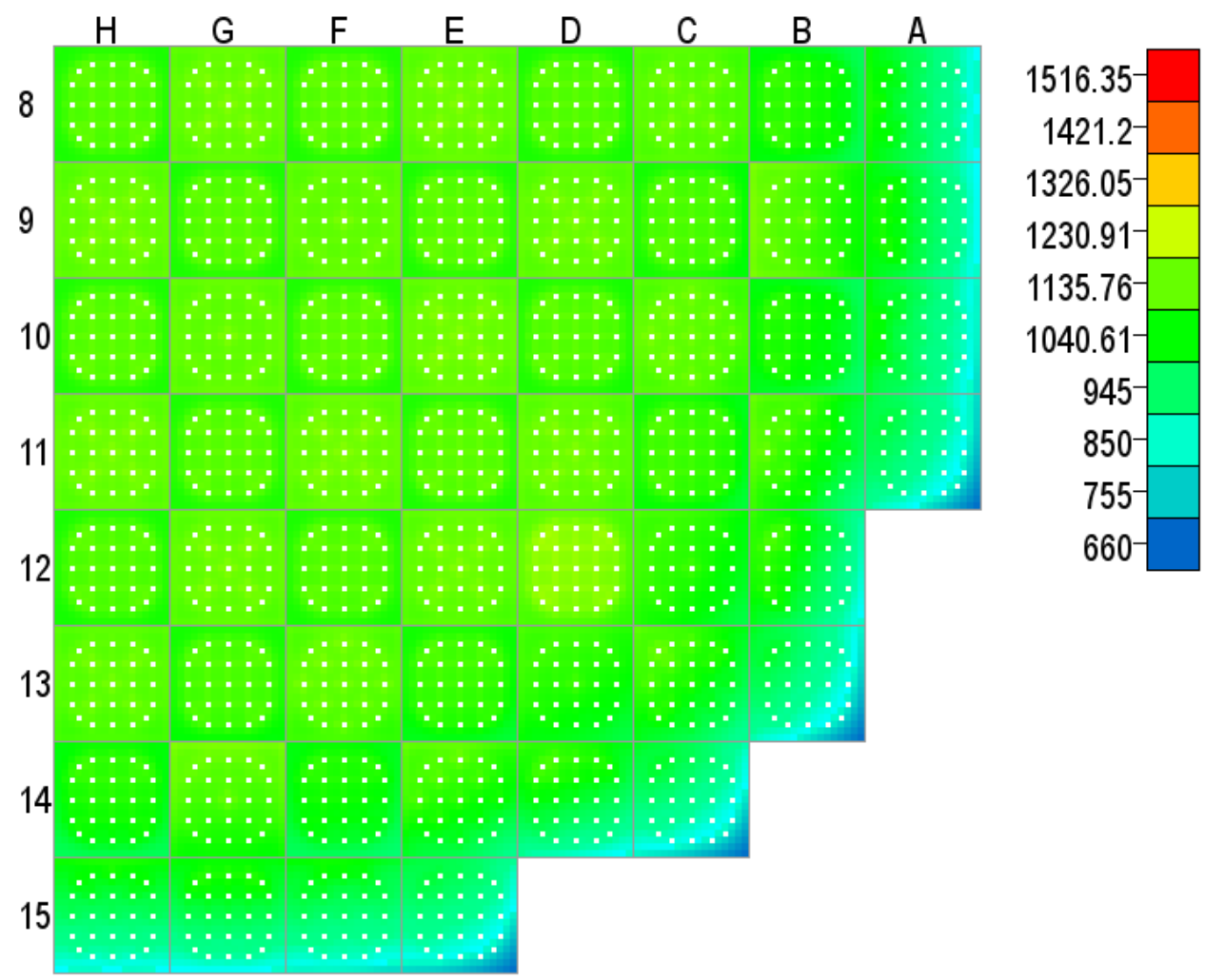

Fig. A.1.20. Cycle 1maximum centerline fuel temperature $(\mathrm{K})-15.10 \mathrm{GWd} / \mathrm{MT}$. 

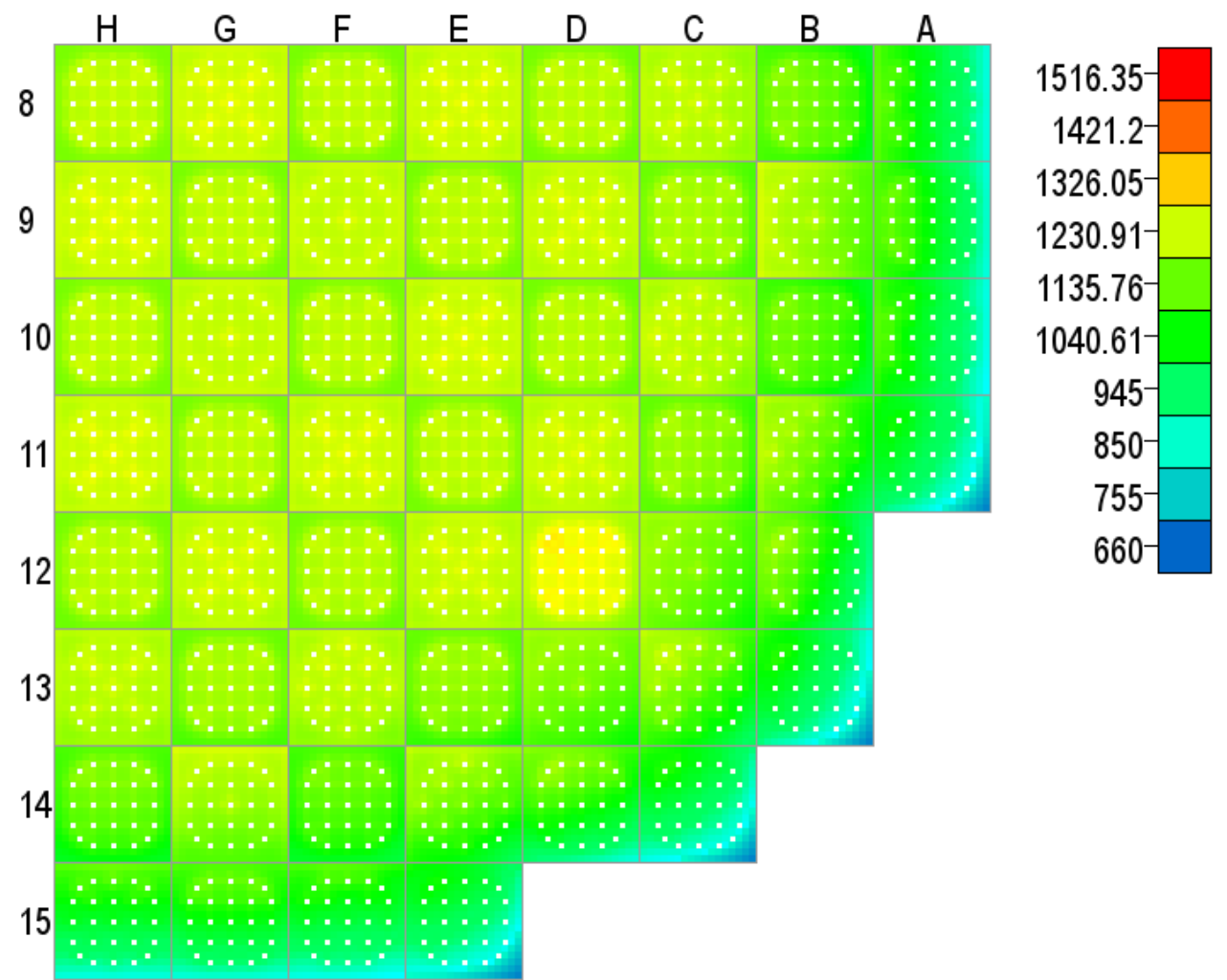

Fig. A.1.21. Cycle 1maximum centerline fuel temperature (K) - 15.30 GWd/MT.

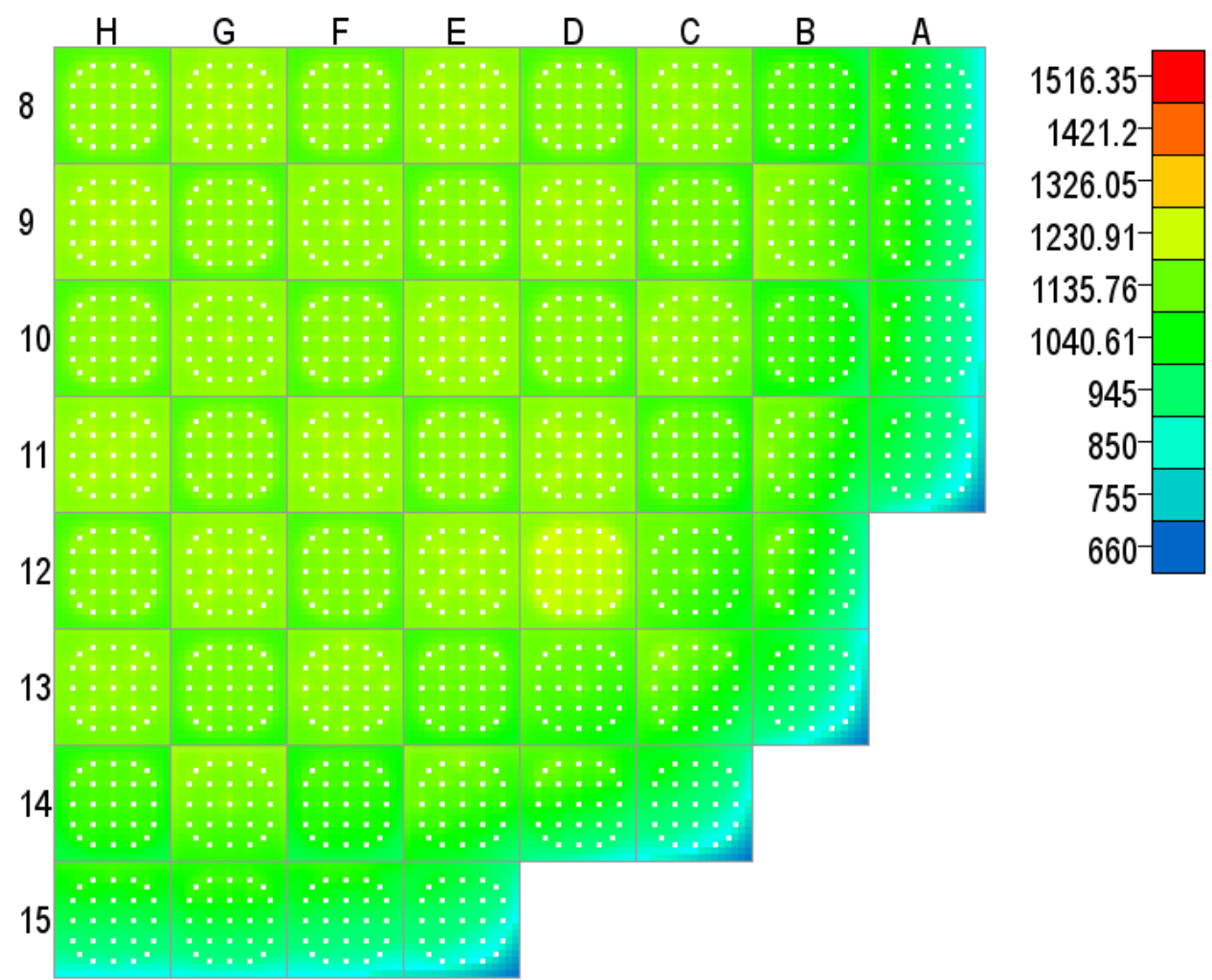

Fig. A.1.22. Cycle 1maximum centerline fuel temperature $(\mathrm{K})-15.80 \mathrm{GWd} / \mathrm{MT}$. 


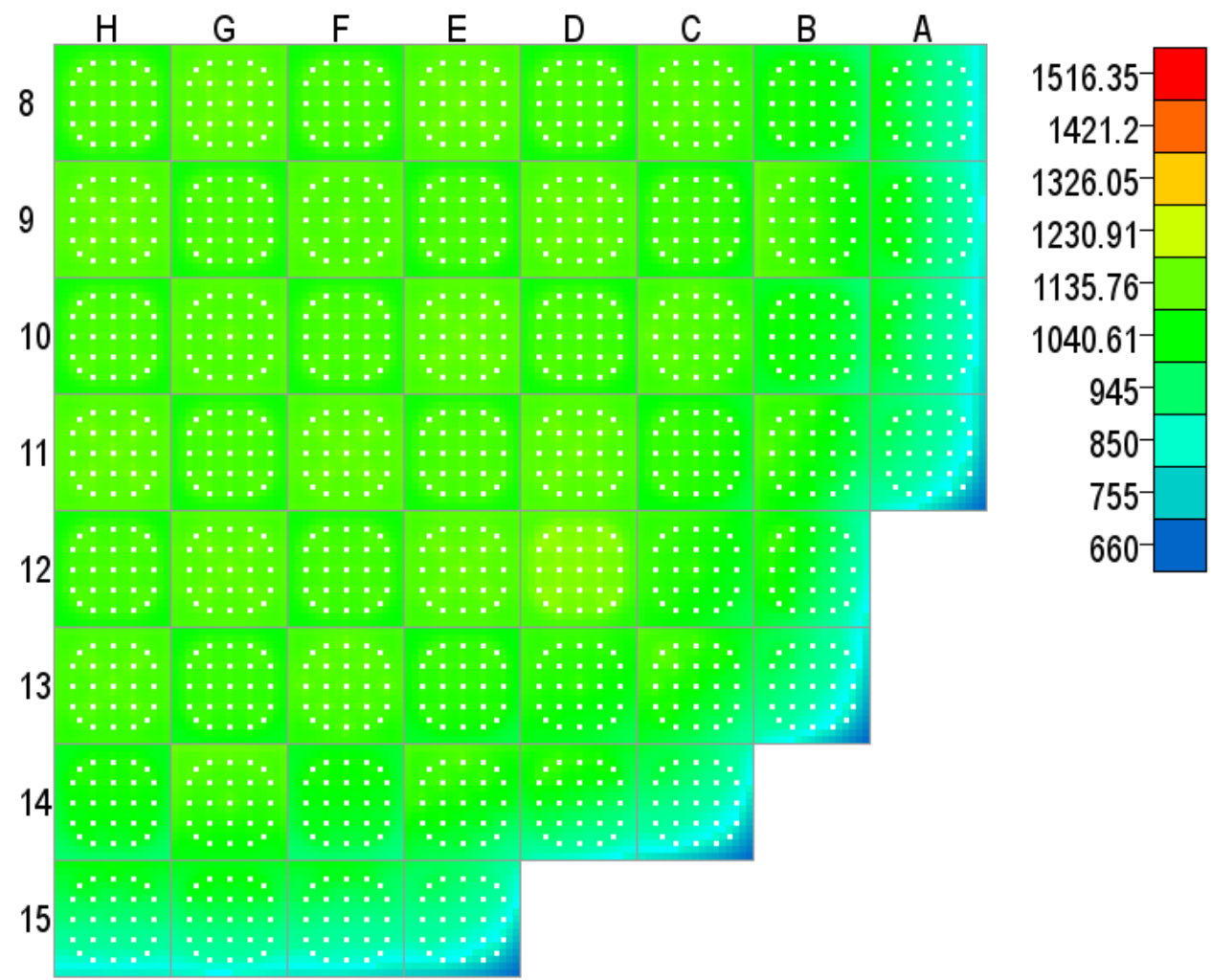

Fig. A.1.23. Cycle 1maximum centerline fuel temperature $(\mathrm{K})$ - 16.30 GWd/MT.

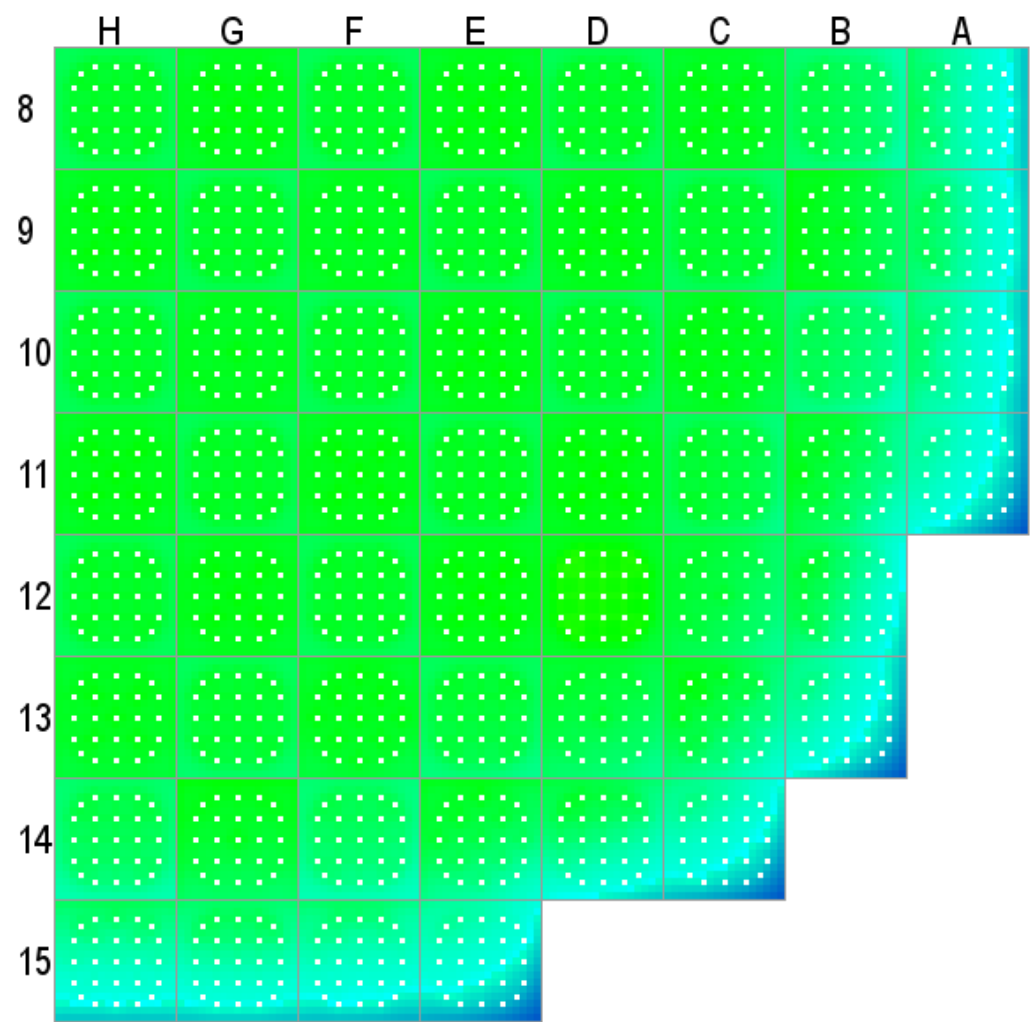

1516.35

1421.2

1326.05

1230.91

1135.76

1040.61

945

850

755

660

Fig. A.1.24. Cycle 1maximum centerline fuel temperature (K) - 16.90 GWd/MT. 


\section{A.1.2. Minimum Gap Thickness}

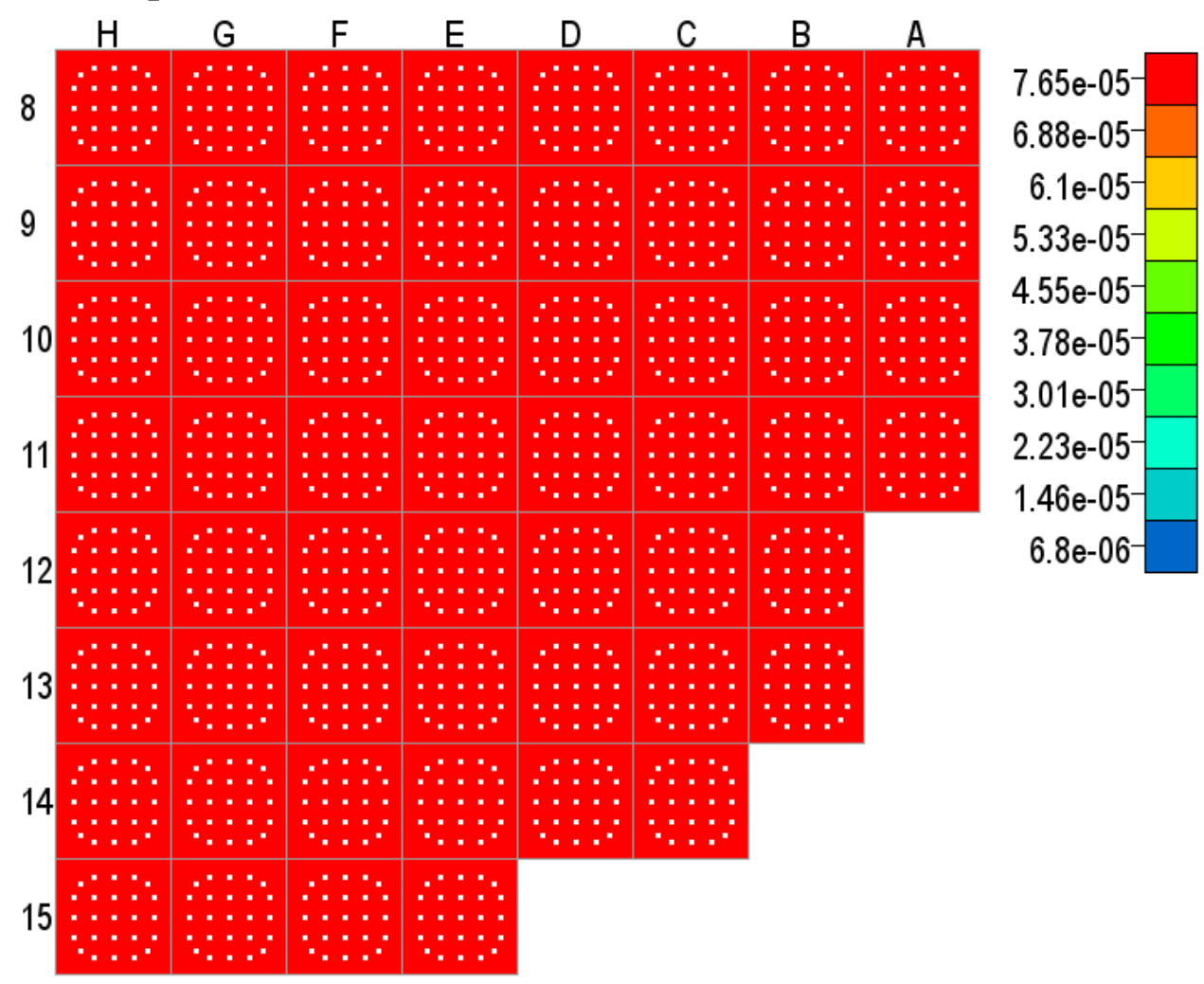

Fig. A.1.25. Cycle 1 minimum gap thickness $(\mathrm{m})-0.00 \mathrm{GWd} / \mathrm{MT}$.

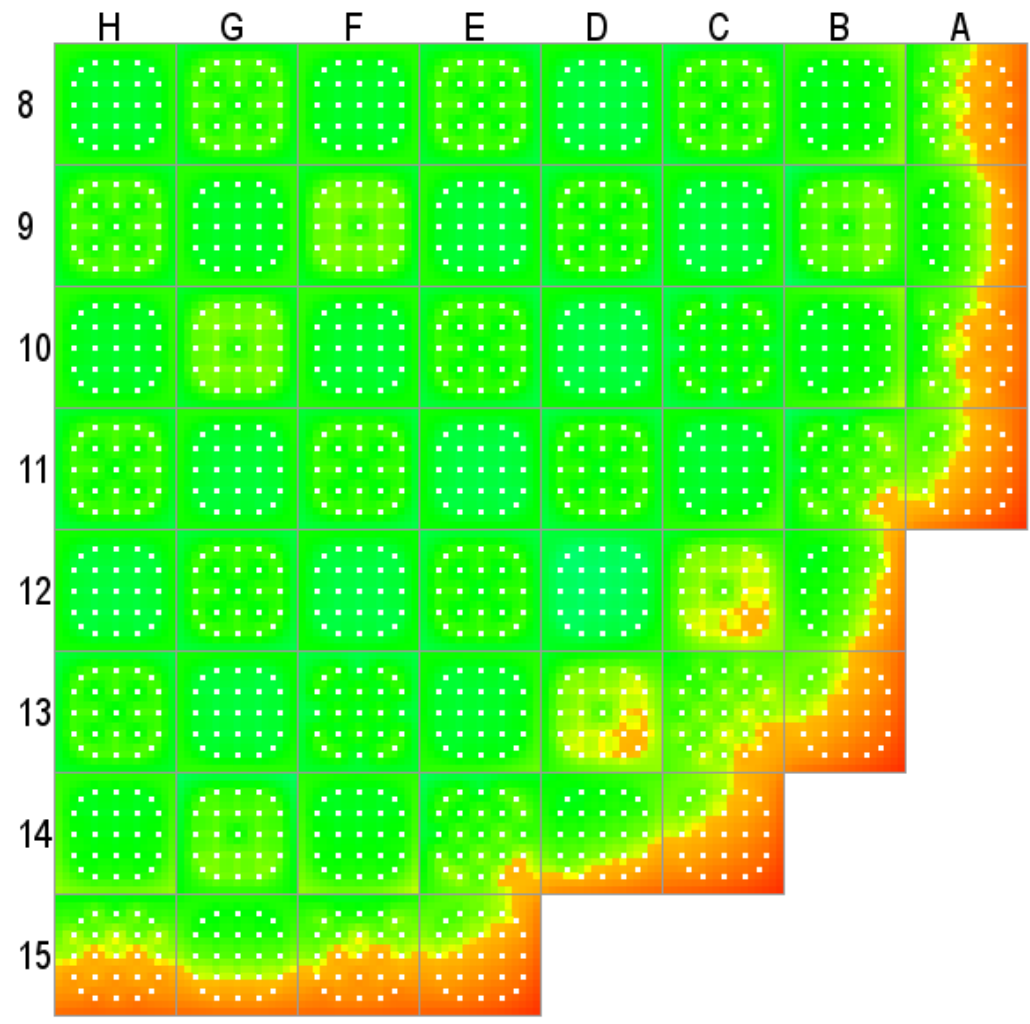

$7.65 \mathrm{e}-05$
$6.88 \mathrm{e}-05-$
$6.1 \mathrm{e}-05$
$5.33 \mathrm{e}-05$
$4.55 \mathrm{e}-05$
$3.78 \mathrm{e}-05$
$3.01 \mathrm{e}-05-$
$2.23 \mathrm{e}-05$
$1.46 \mathrm{e}-05$
$6.8 \mathrm{e}-06$

Fig. A.1.26. Cycle 1 minimum gap thickness $(\mathrm{m})-0.35$ GWd/MT. 


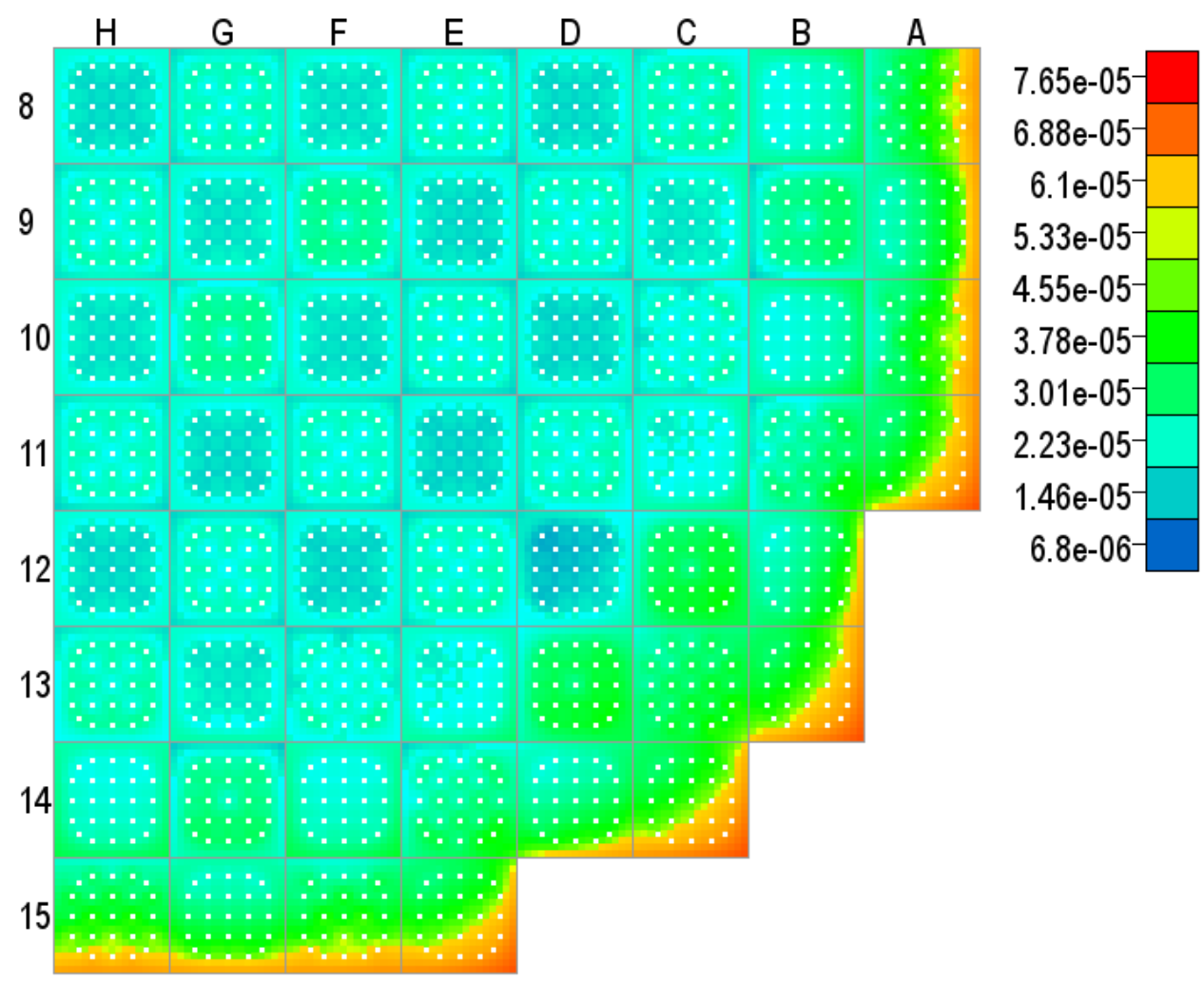

Fig. A.1.27. Cycle 1 minimum gap thickness (m) - 1.23 GWd/MT.

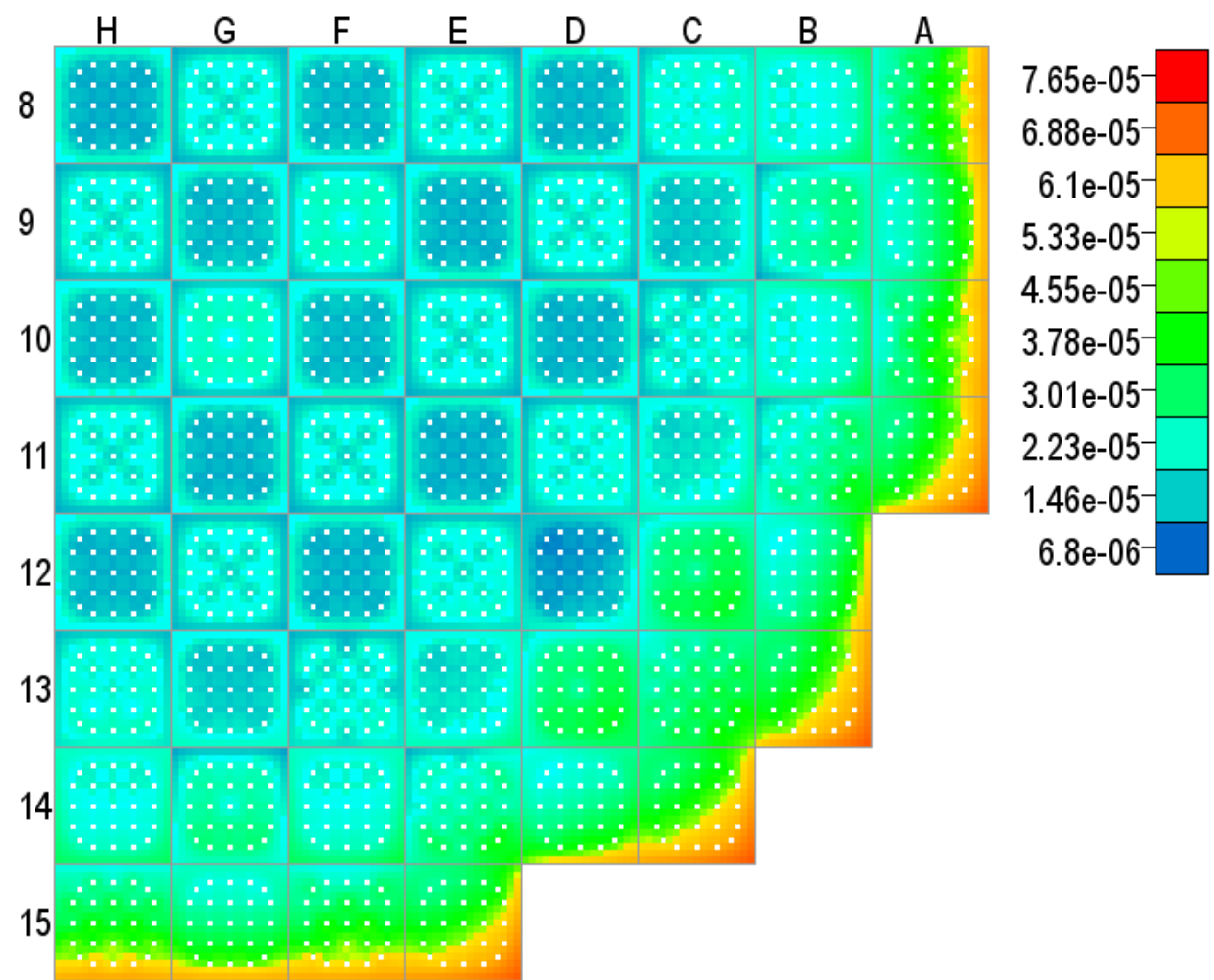

Fig. A.1.28. Cycle 1 minimum gap thickness (m) - 1.92 GWd/MT. 


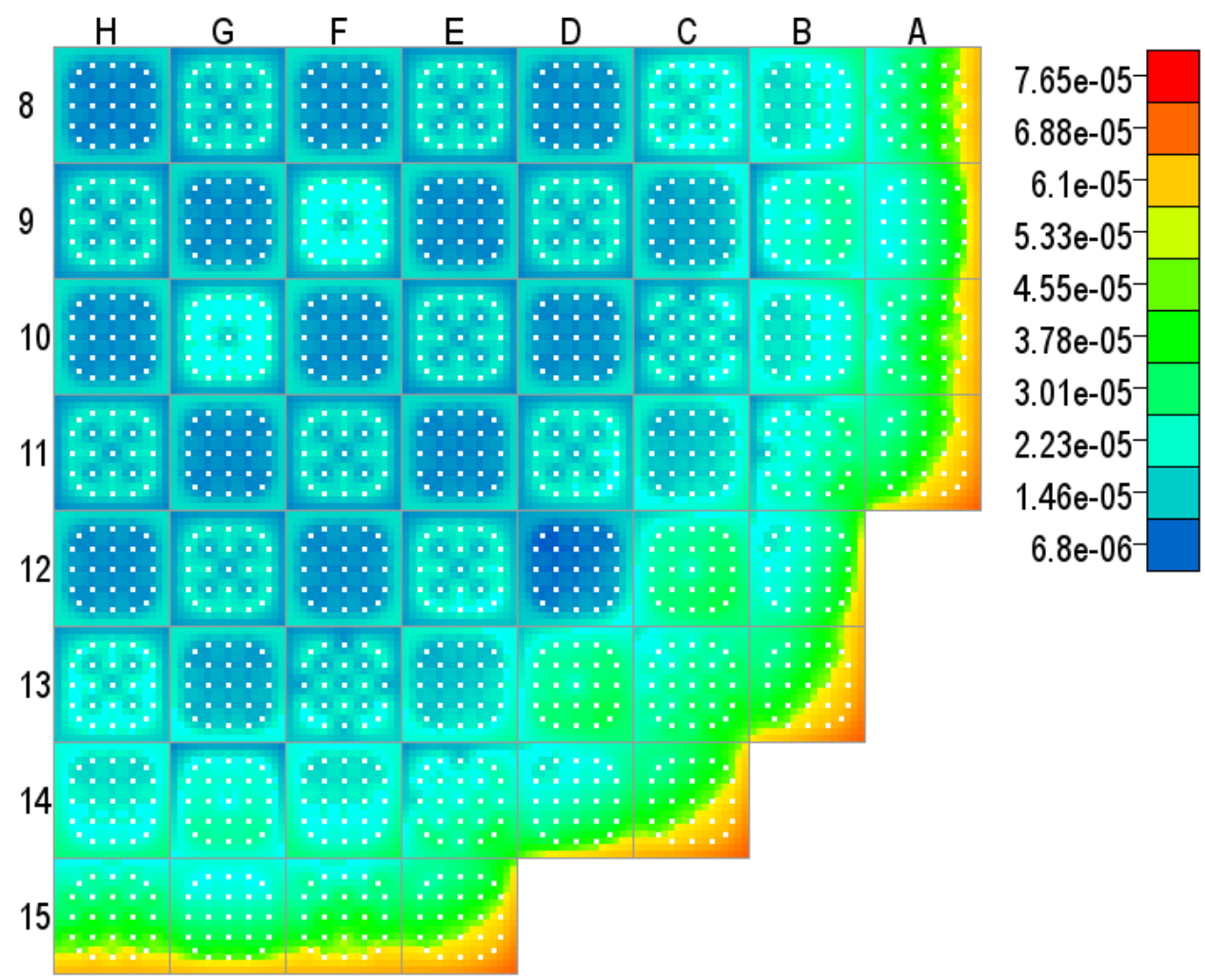

Fig. A.1.29. Cycle 1 minimum gap thickness (m) - 2.46 GWd/MT.

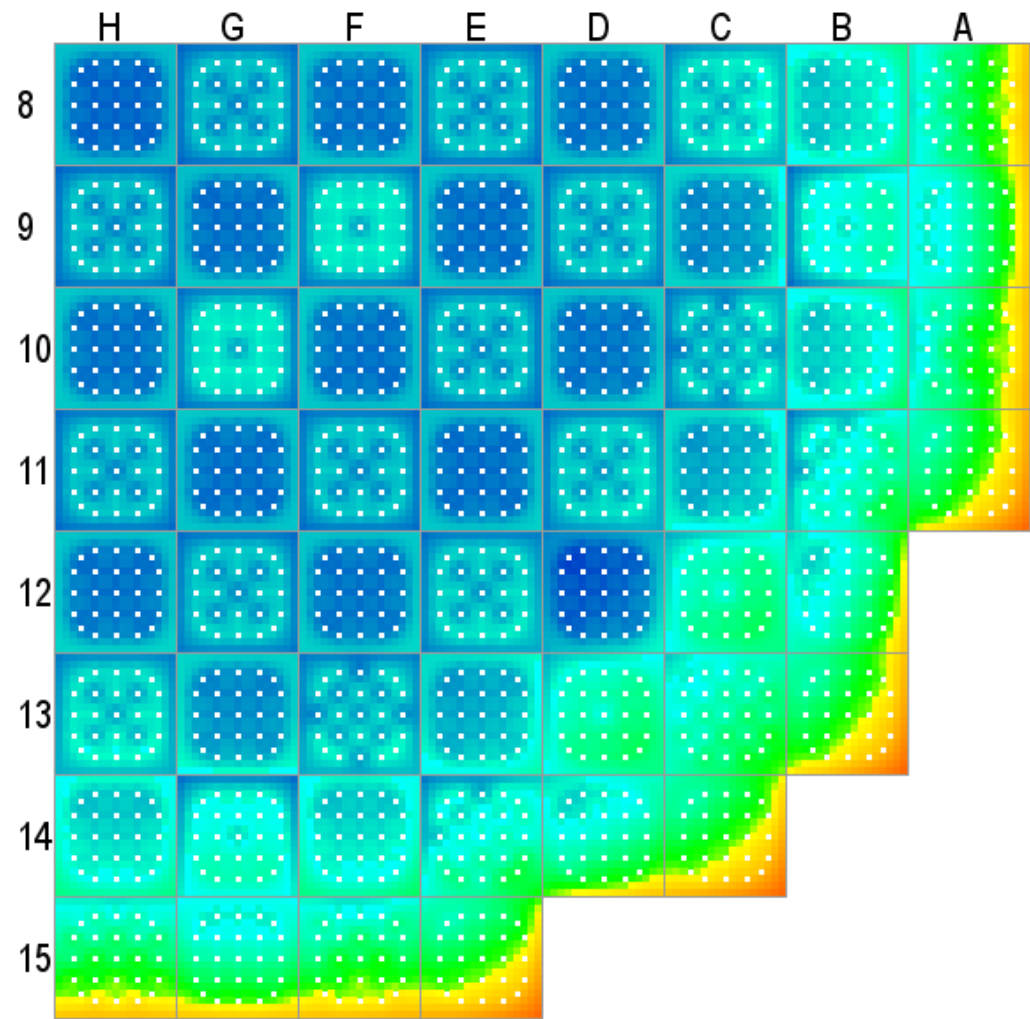

$7.65 \mathrm{e}-05$
$6.88 \mathrm{e}-05$
$6.1 \mathrm{e}-05$
$5.33 \mathrm{e}-05$
$4.55 \mathrm{e}-05$
$3.78 \mathrm{e}-05$
$3.01 \mathrm{e}-05$
$2.23 \mathrm{e}-05$
$1.46 \mathrm{e}-05$
$6.8 \mathrm{e}-06$

Fig. A.1.30. Cycle 1 minimum gap thickness (m) - 2.99 GWd/MT. 


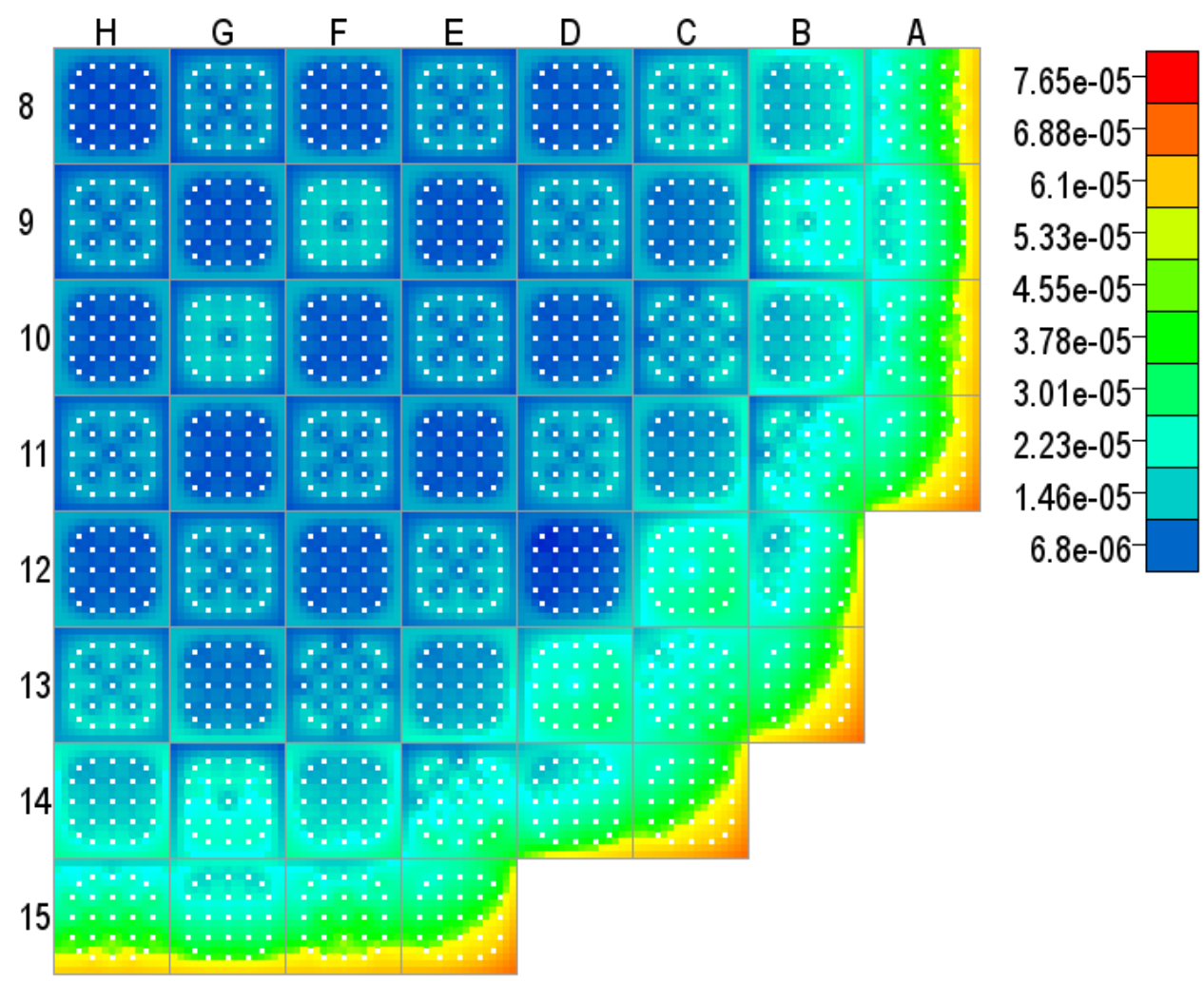

Fig. A.1.31. Cycle 1 minimum gap thickness (m) - 3.56 GWd/MT.

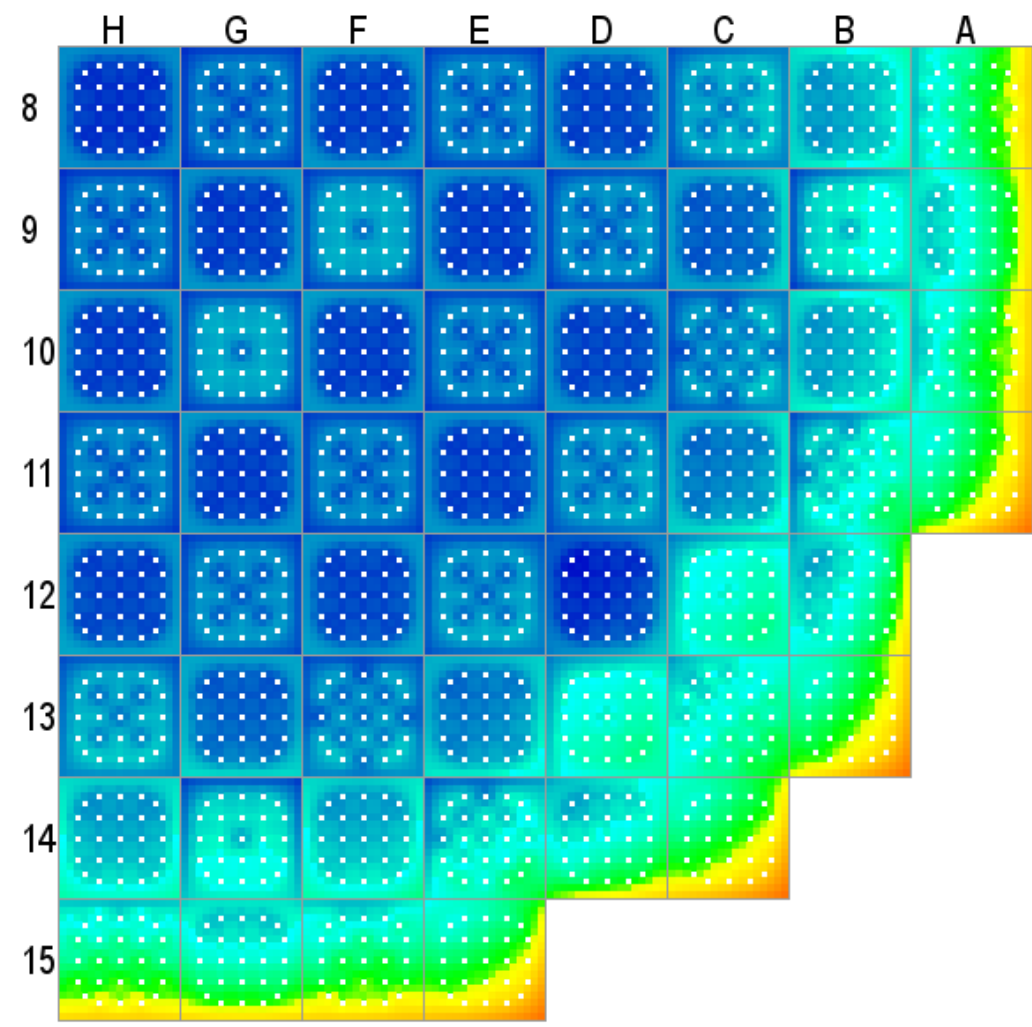

$7.65 \mathrm{e}-05-$
$6.88 \mathrm{e}-05$
$6.1 \mathrm{e}-05-$
$5.33 \mathrm{e}-05$
$4.55 \mathrm{e}-05$
$3.78 \mathrm{e}-05$
$3.01 \mathrm{e}-05$
$2.23 \mathrm{e}-05$
$1.46 \mathrm{e}-05$
$6.8 \mathrm{e}-06$

Fig. A.1.32. Cycle 1 minimum gap thickness $(\mathrm{m})-4.07 \mathrm{GWd} / \mathrm{MT}$. 


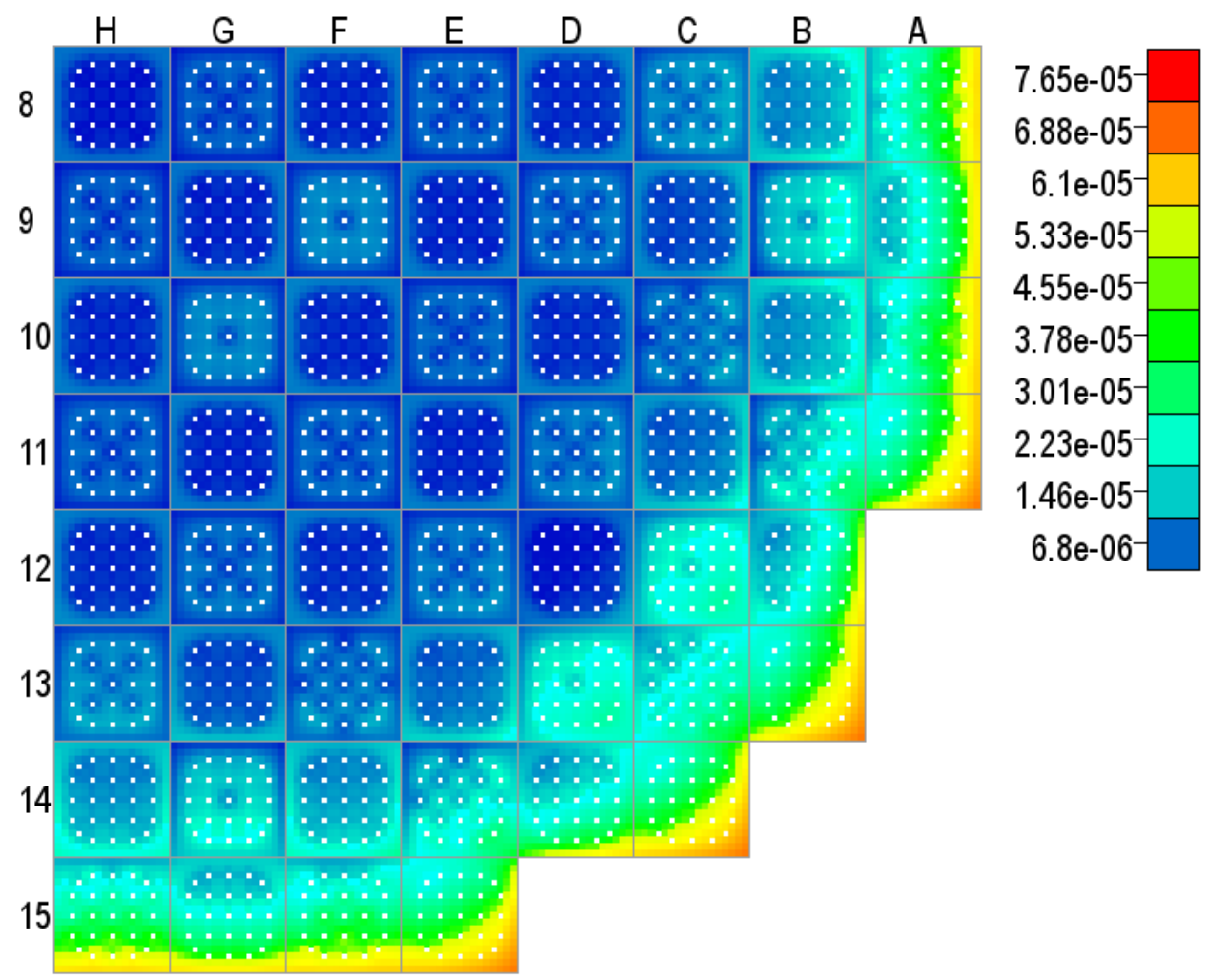

Fig. A.1.33. Cycle 1 minimum gap thickness (m) - 4.64 GWd/MT.

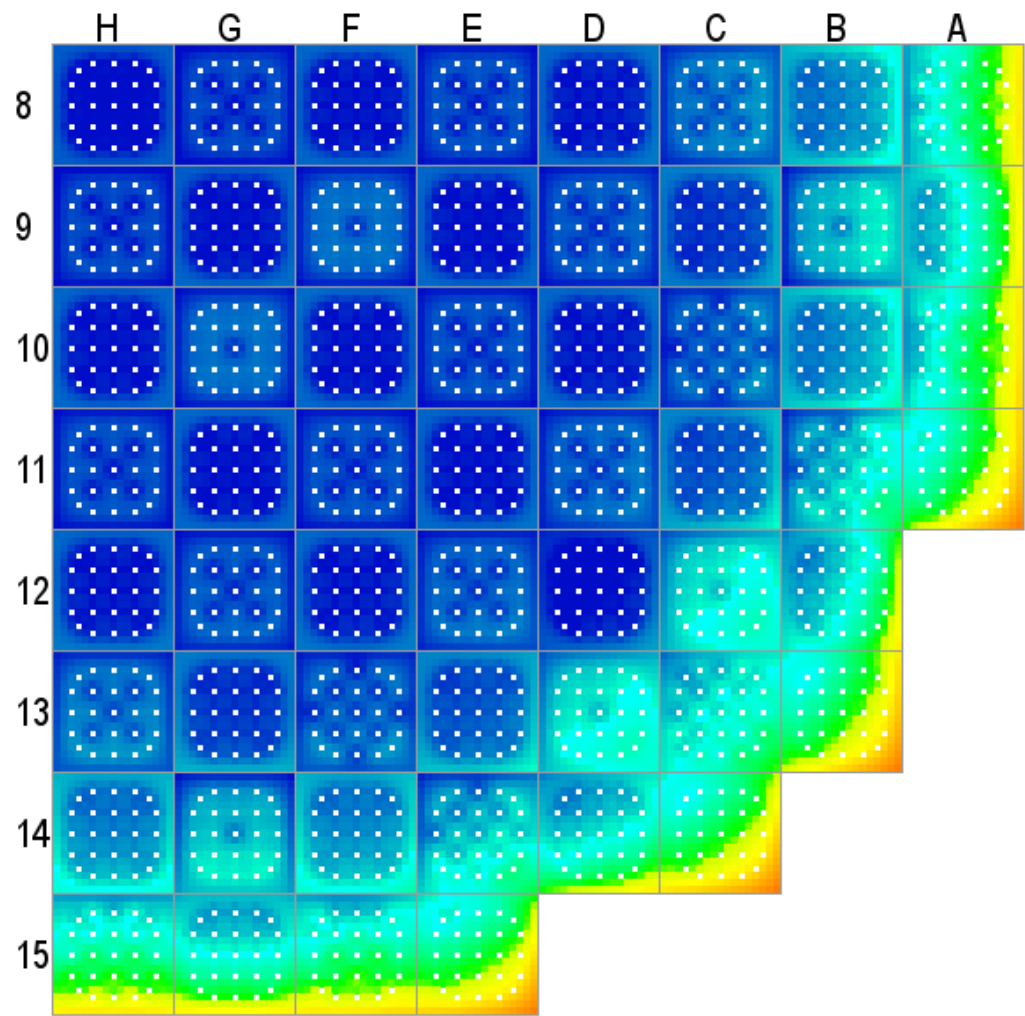

$7.65 \mathrm{e}-05-$
$6.88 \mathrm{e}-05-$
$6.1 \mathrm{e}-05$
$5.33 \mathrm{e}-05$
$4.55 \mathrm{e}-05$
$3.78 \mathrm{e}-05$
$3.01 \mathrm{e}-05$
$2.23 \mathrm{e}-05$
$1.46 \mathrm{e}-05$
$6.8 \mathrm{e}-06-$

Fig. A.1.34. Cycle 1 minimum gap thickness (m) - 5.14 GWd/MT. 


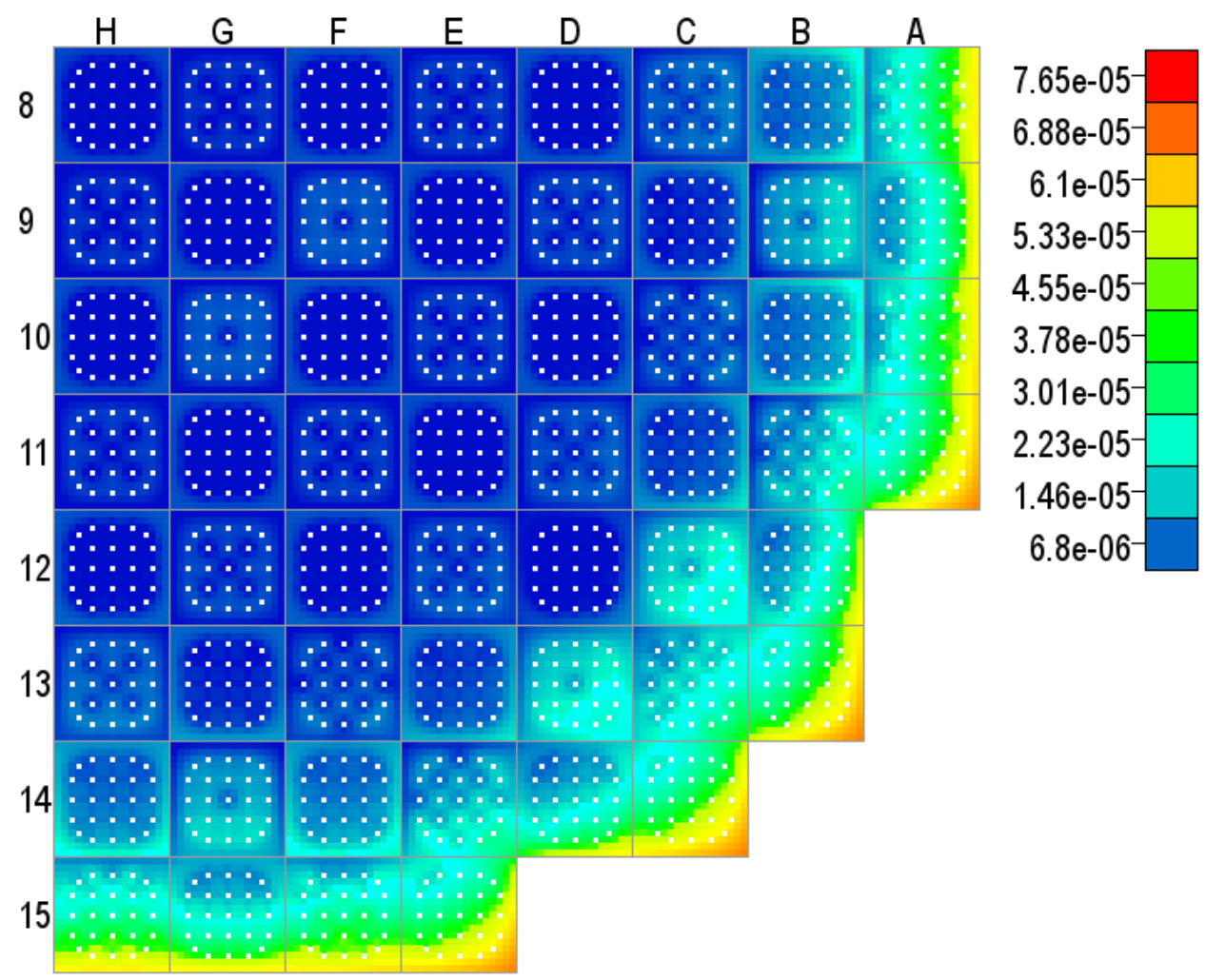

Fig. A.1.35. Cycle 1 minimum gap thickness (m) - 5.70 GWd/MT.

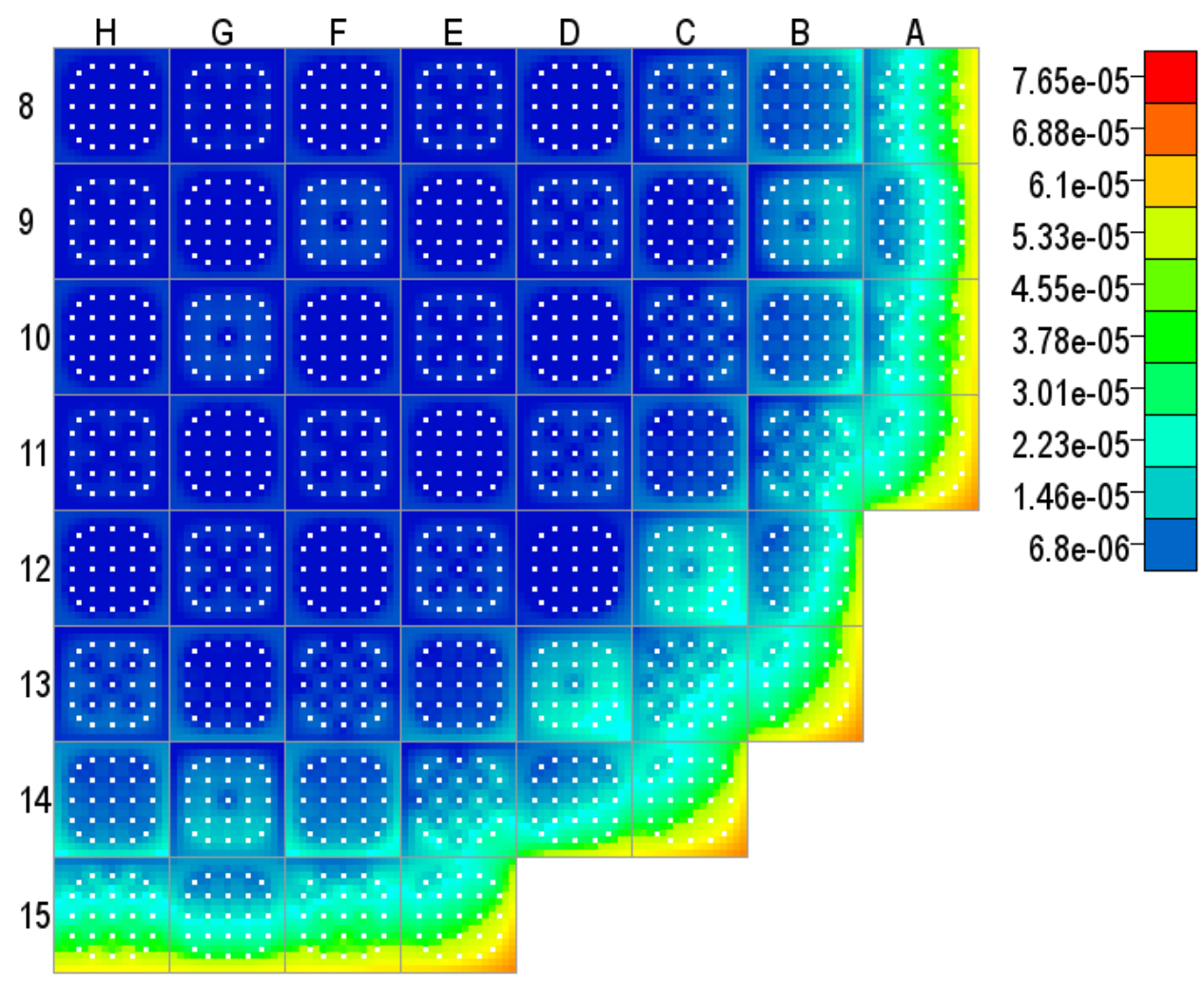

Fig. A.1.36. Cycle 1 minimum gap thickness (m) - 6.27 GWd/MT. 


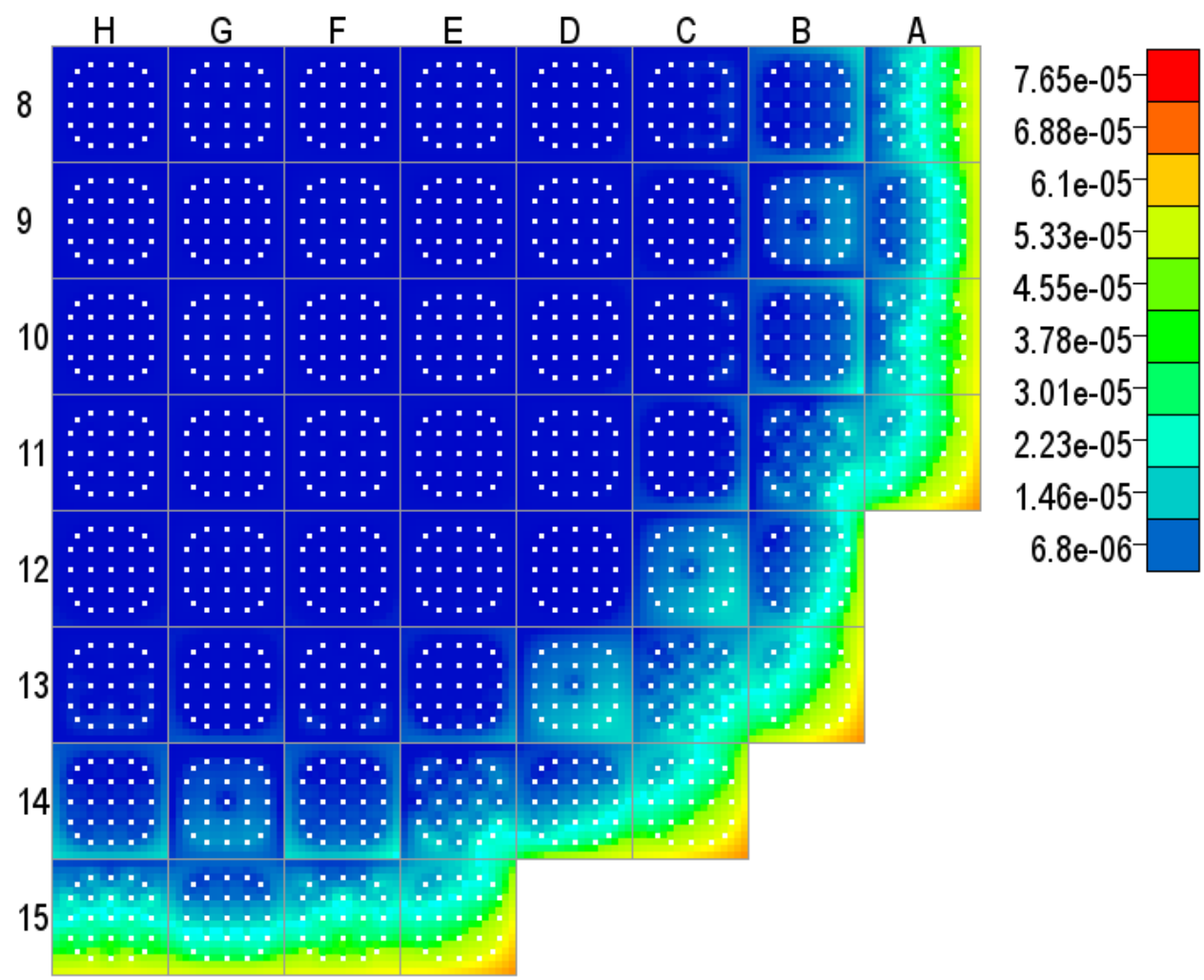

Fig. A.1.37. Cycle 1 minimum gap thickness (m) - 7.46 GWd/MT.

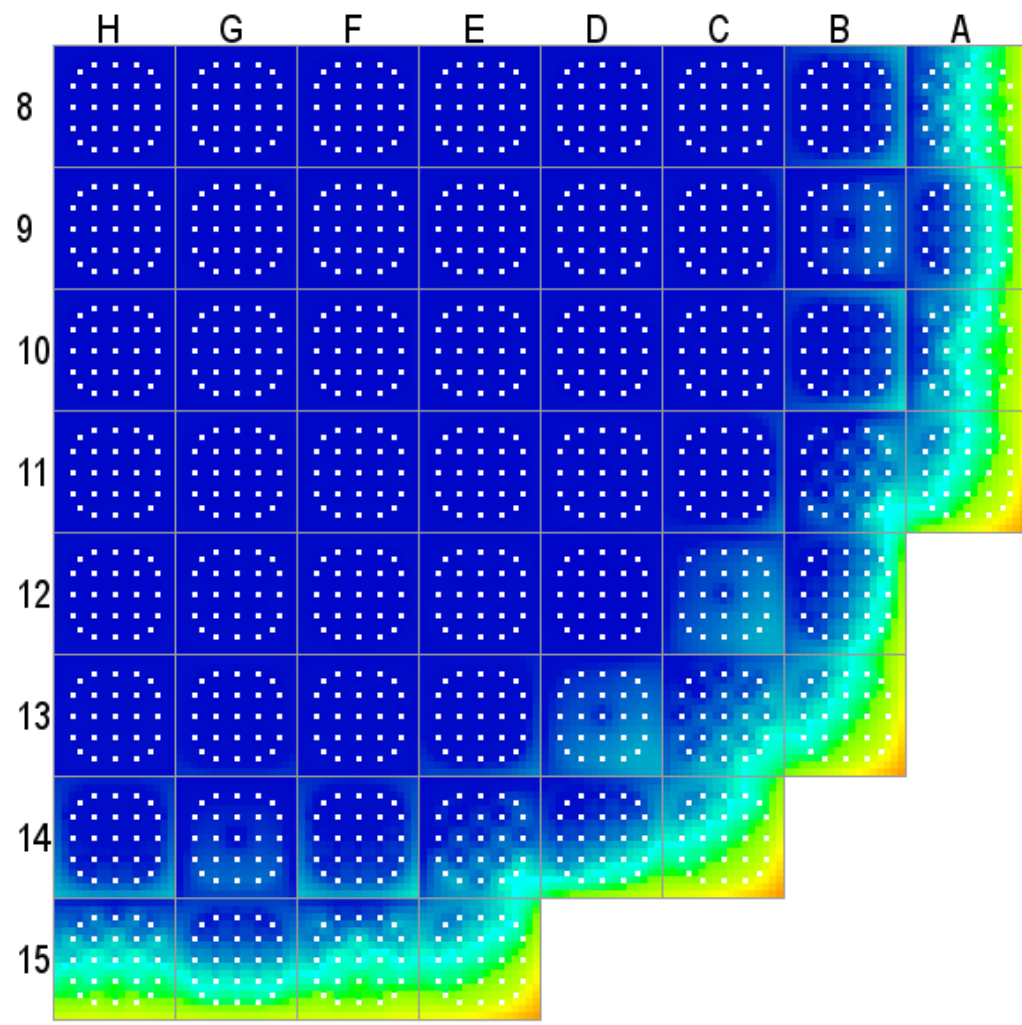

$7.65 \mathrm{e}-05$
$6.88 \mathrm{e}-05$
$6.1 \mathrm{e}-05$
$5.33 \mathrm{e}-05$
$4.55 \mathrm{e}-05$
$3.78 \mathrm{e}-05$
$3.01 \mathrm{e}-05$
$2.23 \mathrm{e}-05$
$1.46 \mathrm{e}-05$
$6.8 \mathrm{e}-06$

Fig. A.1.38. Cycle 1 minimum gap thickness (m) - 8.49 GWd/MT. 


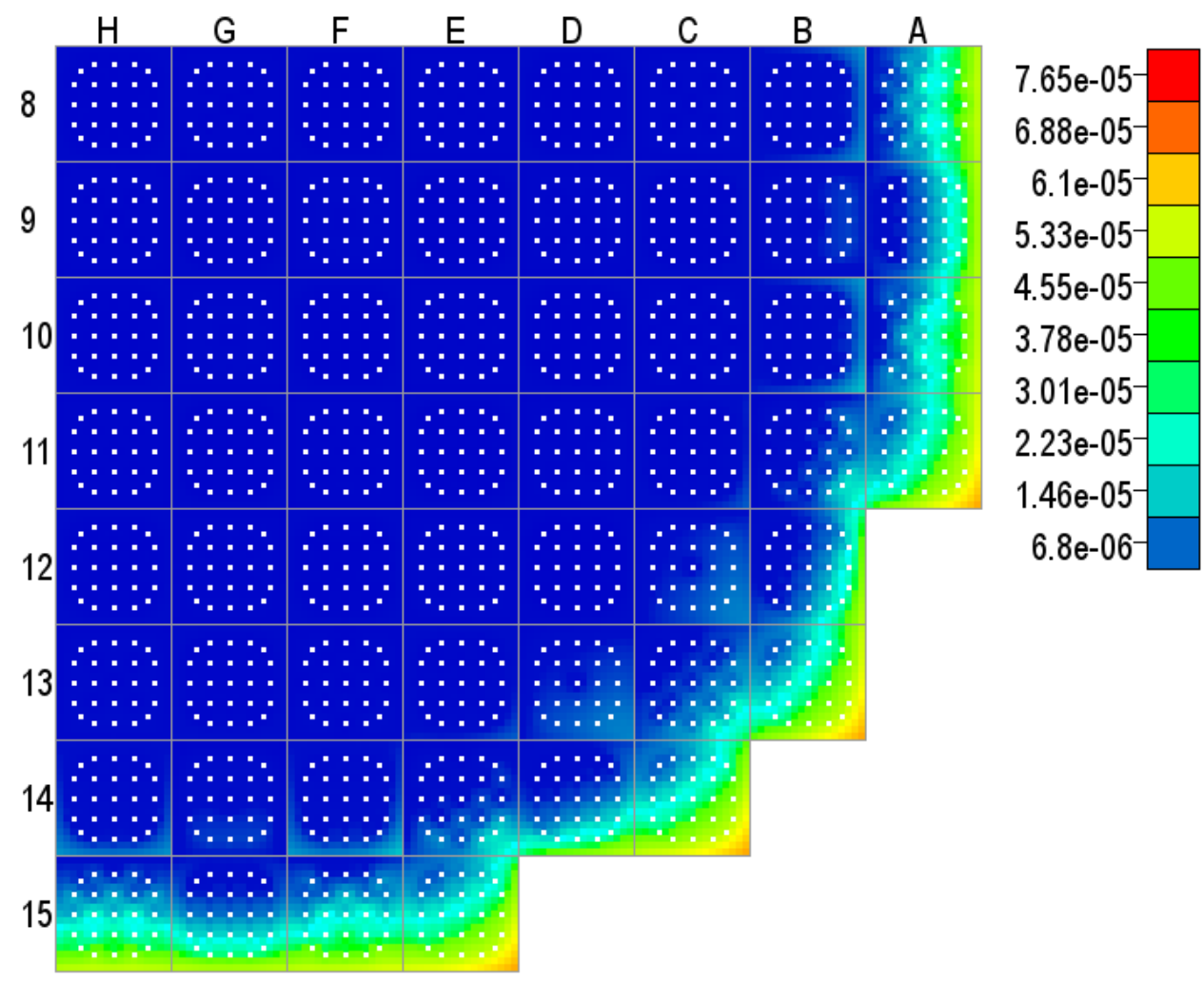

Fig. A.1.39. Cycle 1 minimum gap thickness (m) - 9.60 GWd/MT.

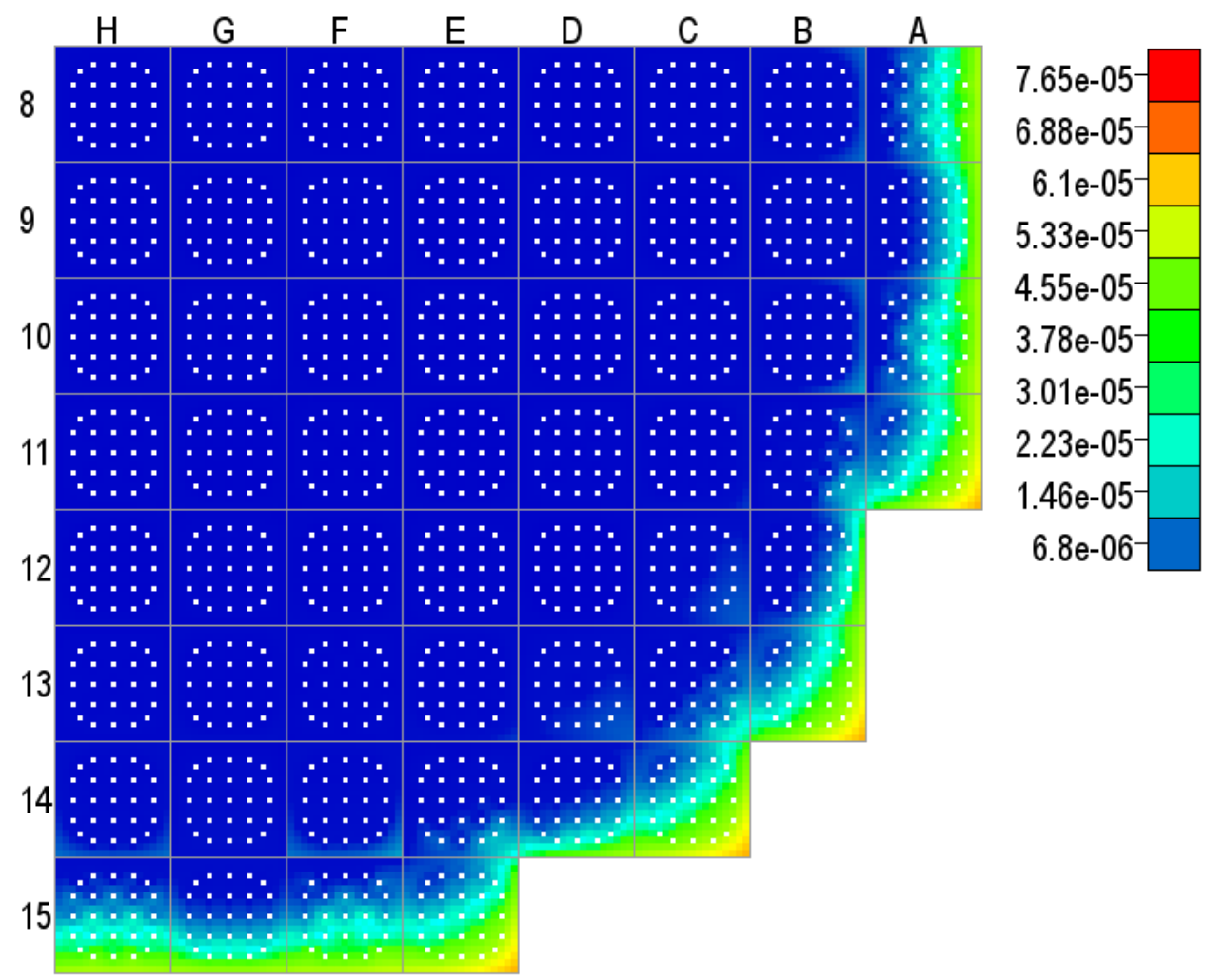

Fig. A.1.40. Cycle 1 minimum gap thickness (m) - 10.8 GWd/MT. 


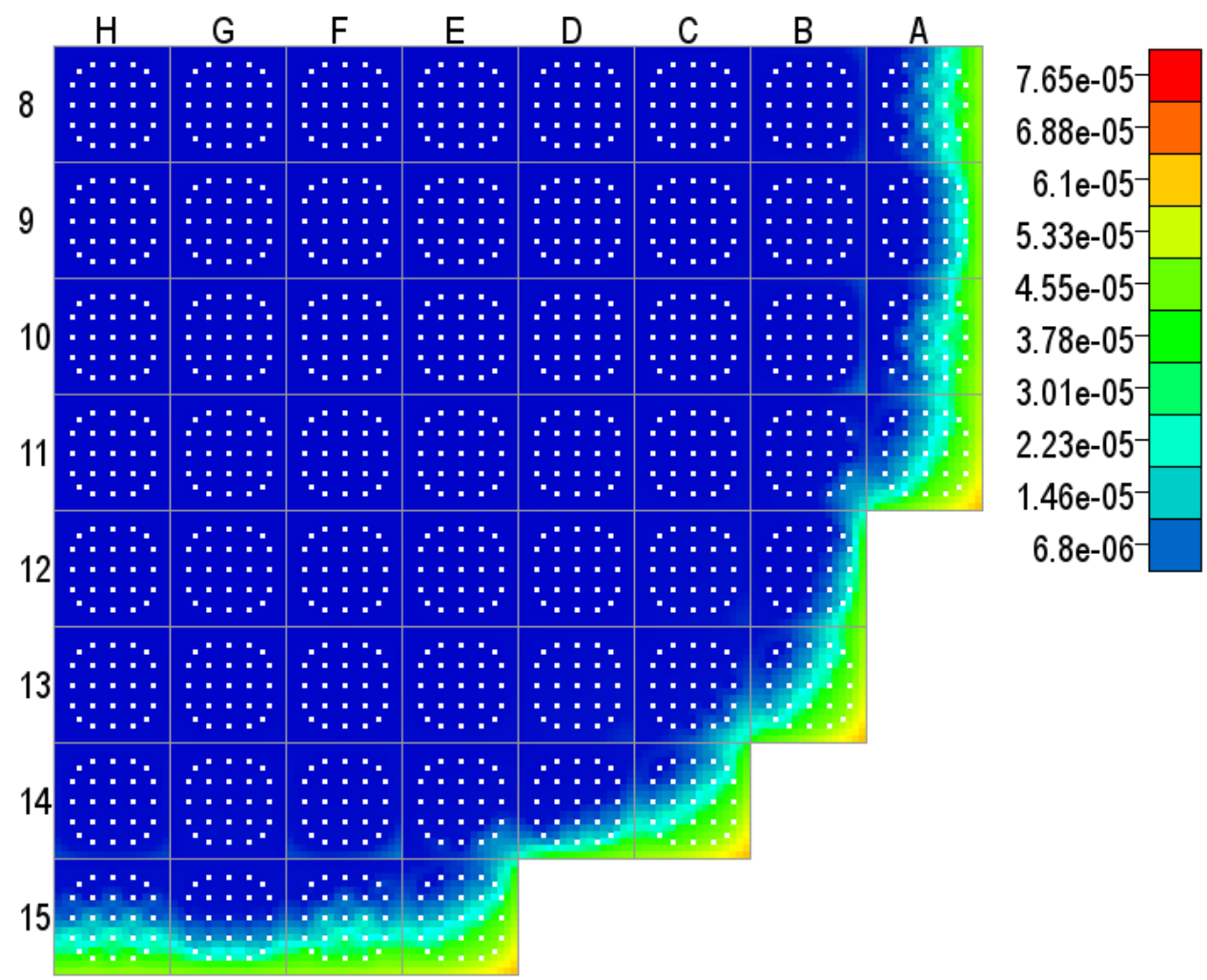

Fig. A.1.41. Cycle 1 minimum gap thickness (m) - 12.00 GWd/MT.

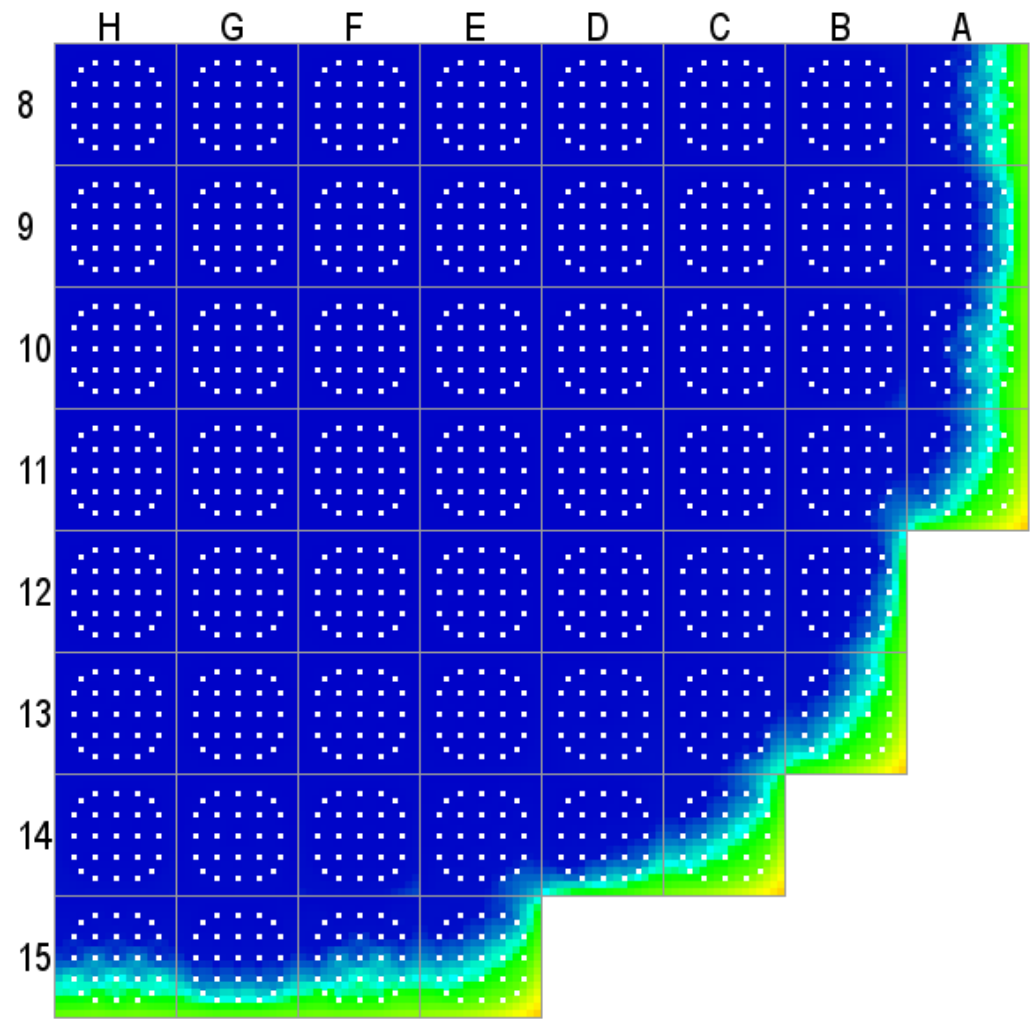

$7.65 \mathrm{e}-05$
$6.88 \mathrm{e}-05$
$6.1 \mathrm{e}-05$
$5.33 \mathrm{e}-05$
$4.55 \mathrm{e}-05$
$3.78 \mathrm{e}-05$
$3.01 \mathrm{e}-05$
$2.23 \mathrm{e}-05$
$1.46 \mathrm{e}-05$
$6.8 \mathrm{e}-06$

Fig. A.1.42. Cycle 1 minimum gap thickness (m) - 13.40 GWd/MT. 


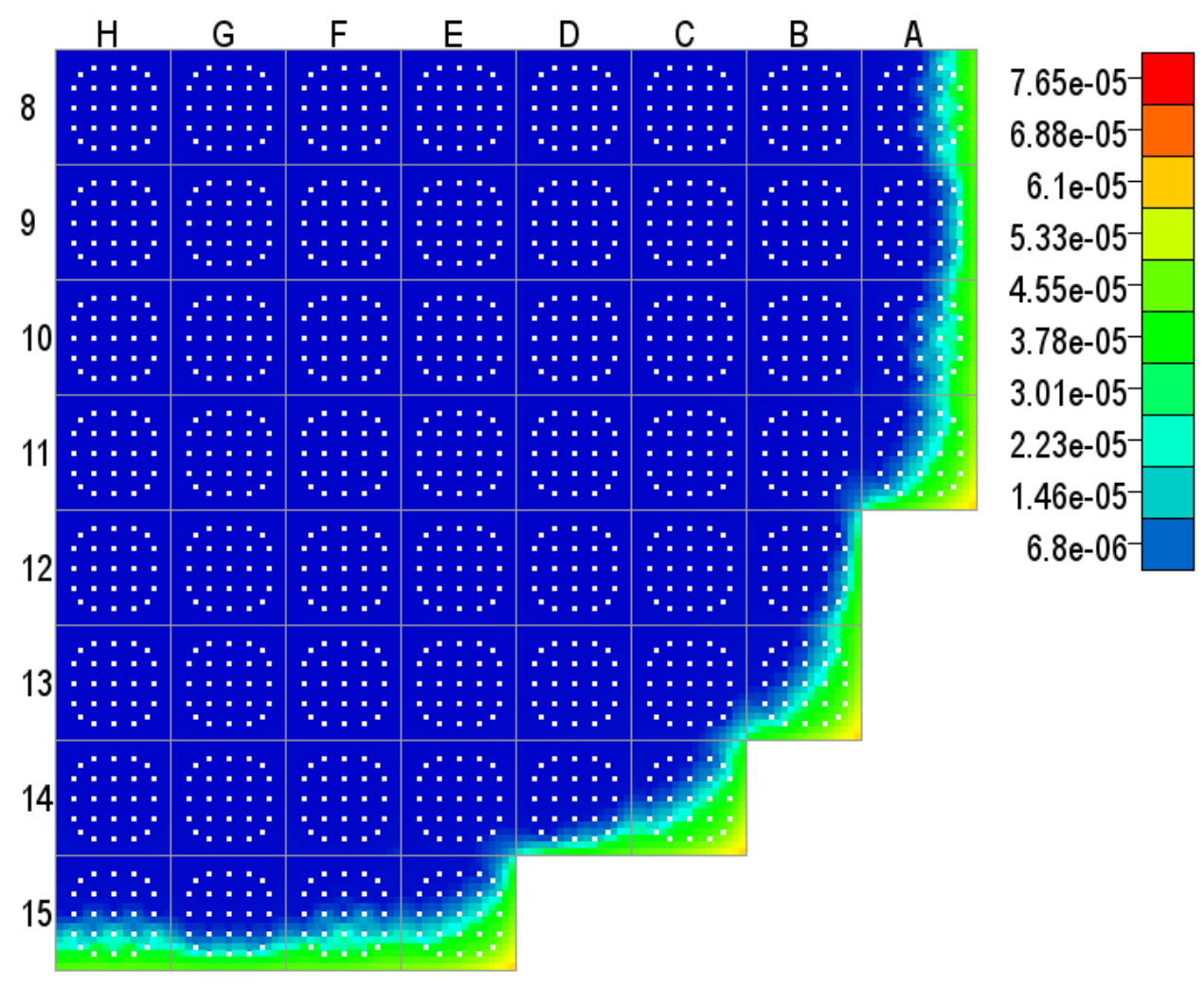

Fig. A.1.43. Cycle 1 minimum gap thickness (m) - 14.3 GWd/MT.

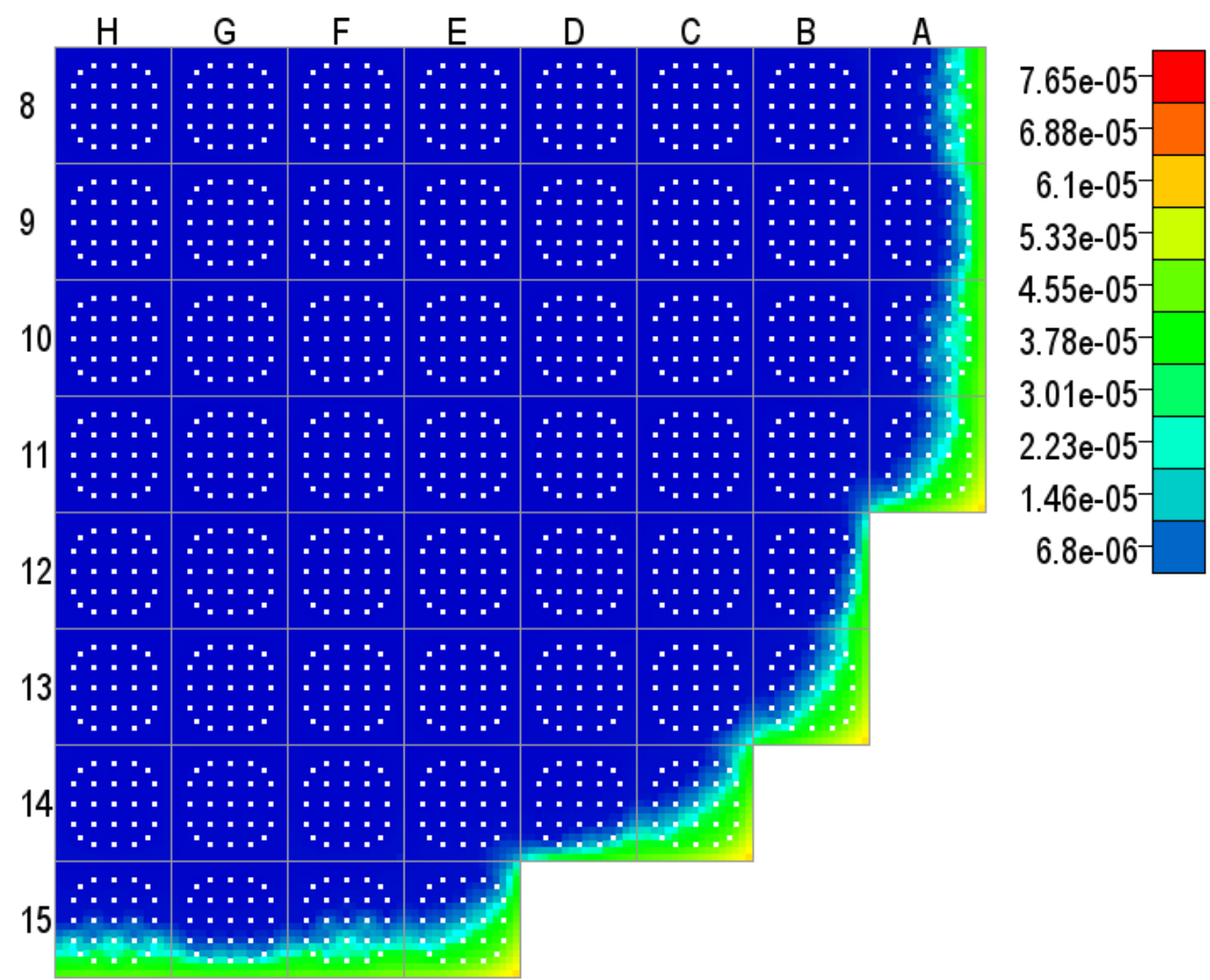

Fig. A.1.44. Cycle 1 minimum gap thickness (m) - 15.10 GWd/MT. 


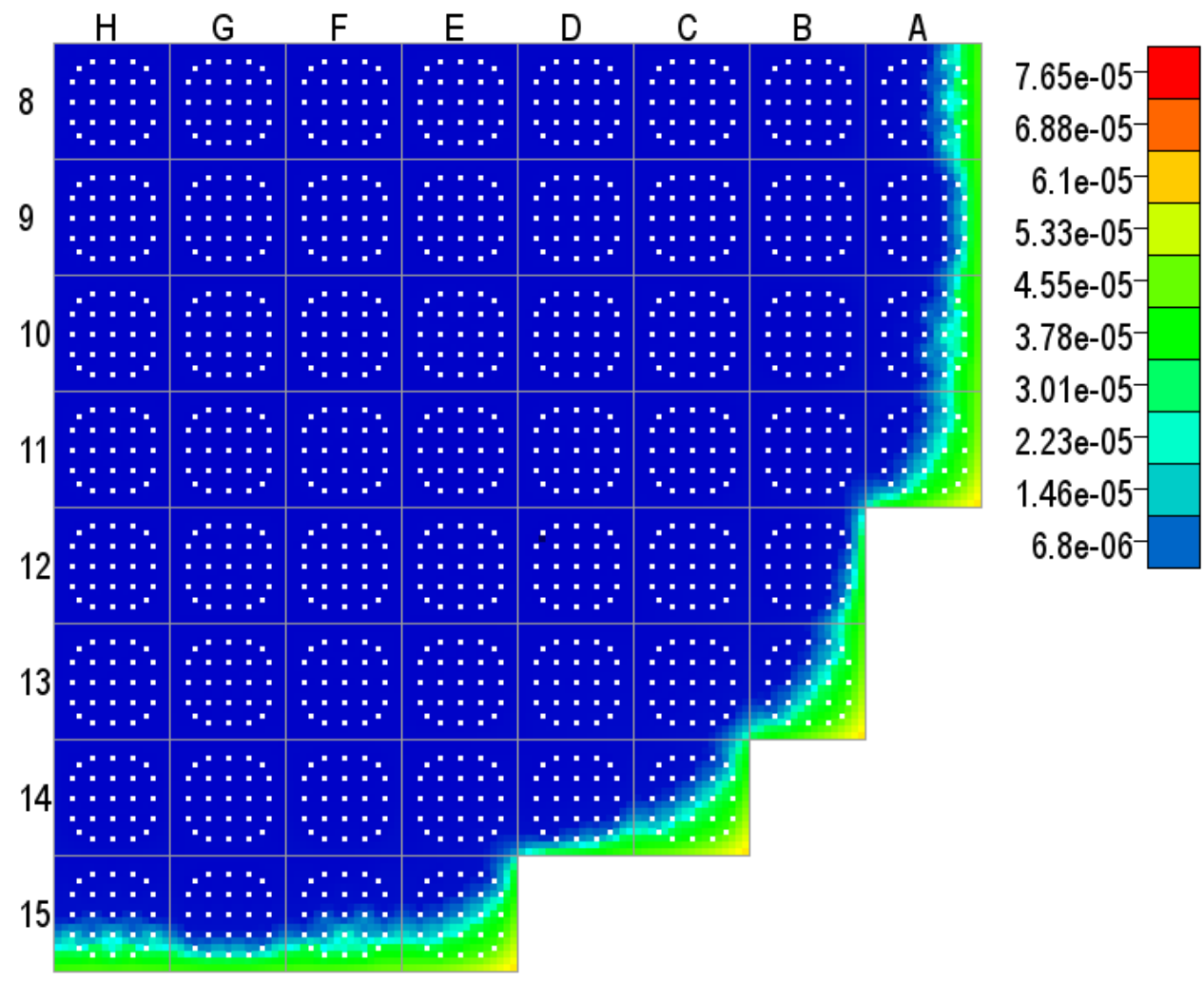

Fig. A.1.45. Cycle 1 minimum gap thickness (m) - 15.30 GWd/MT.

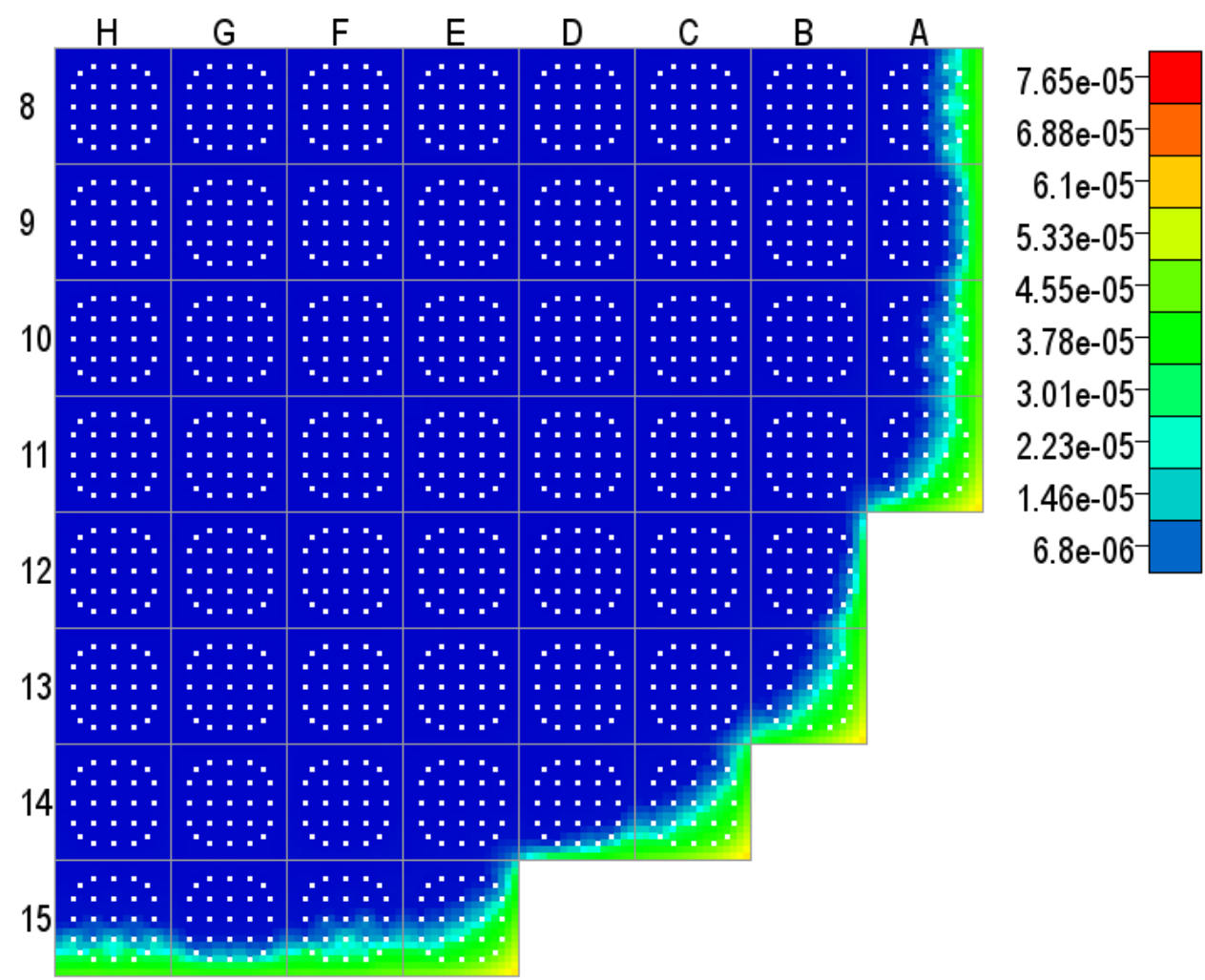

Fig. A.1.46. Cycle 1 minimum gap thickness $(\mathrm{m})-15.80 \mathrm{GWd} / \mathrm{MT}$. 


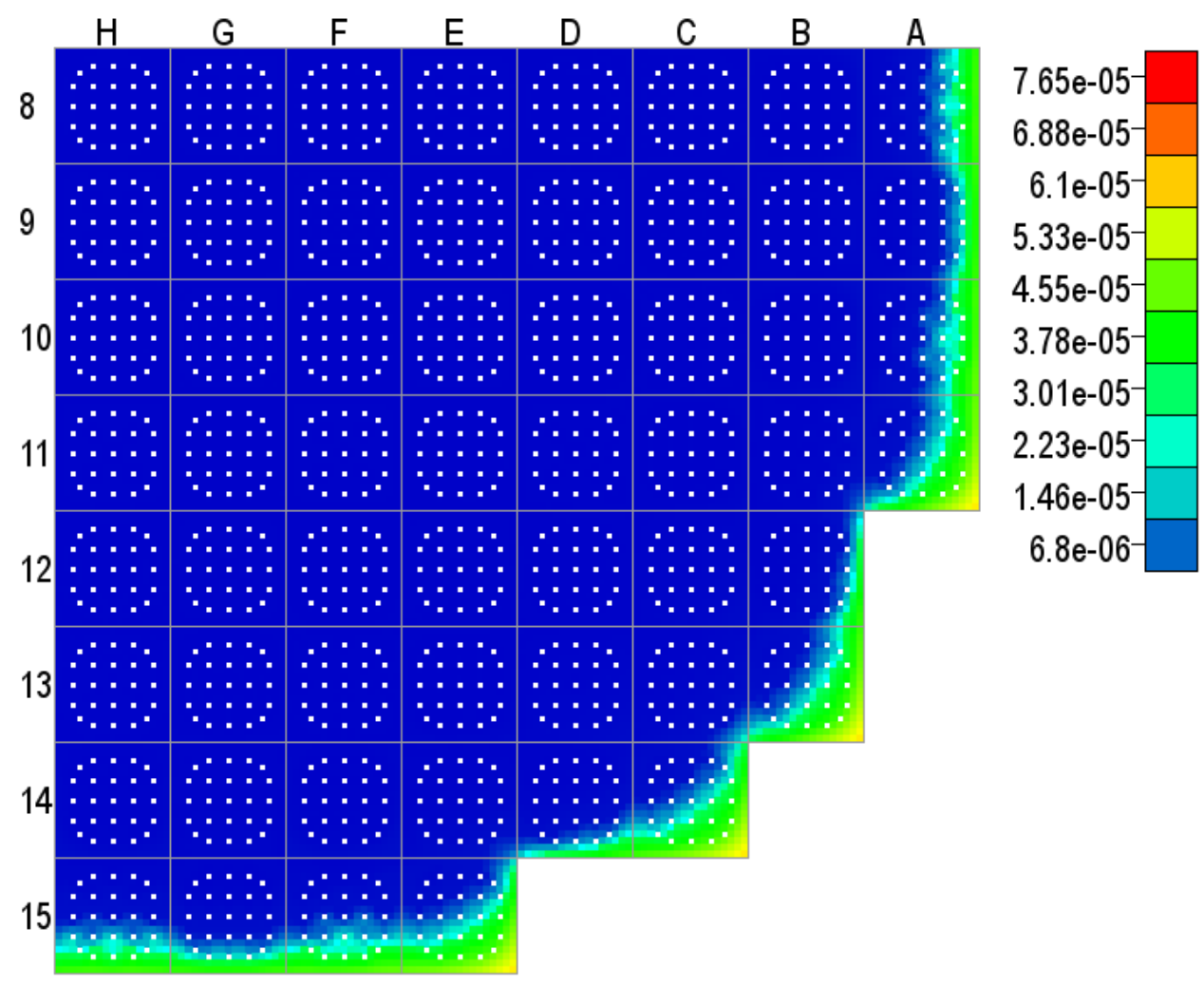

Fig. A.1.47. Cycle 1 minimum gap thickness $(\mathrm{m})-16.30 \mathrm{GWd} / \mathrm{MT}$.

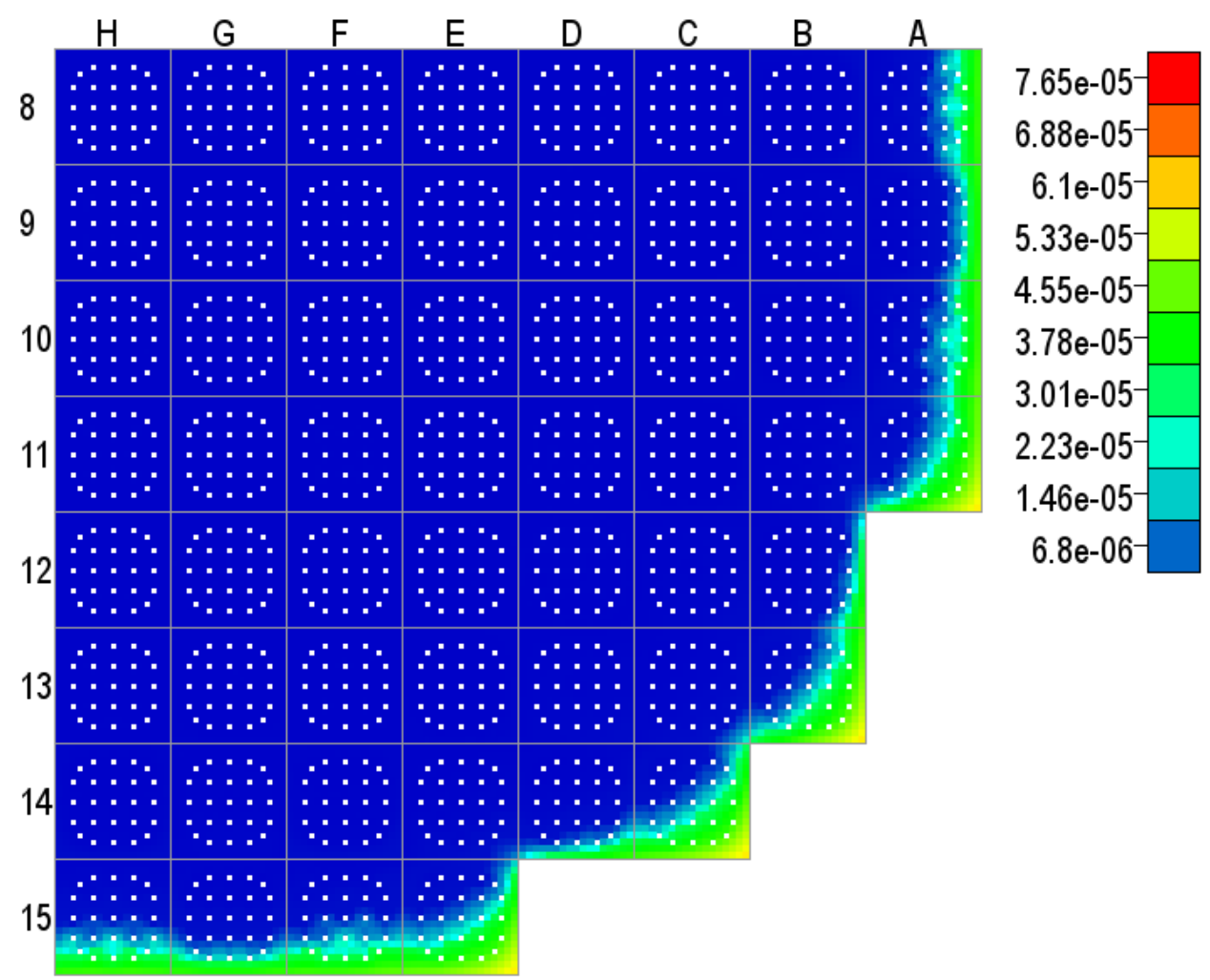

Fig. A.1.48. Cycle 1 minimum gap thickness (m) - 16.90 GWd/MT. 


\section{A.2. Cycle 2}

\section{A.2.1. Maximum Centerline Fuel Temperature}

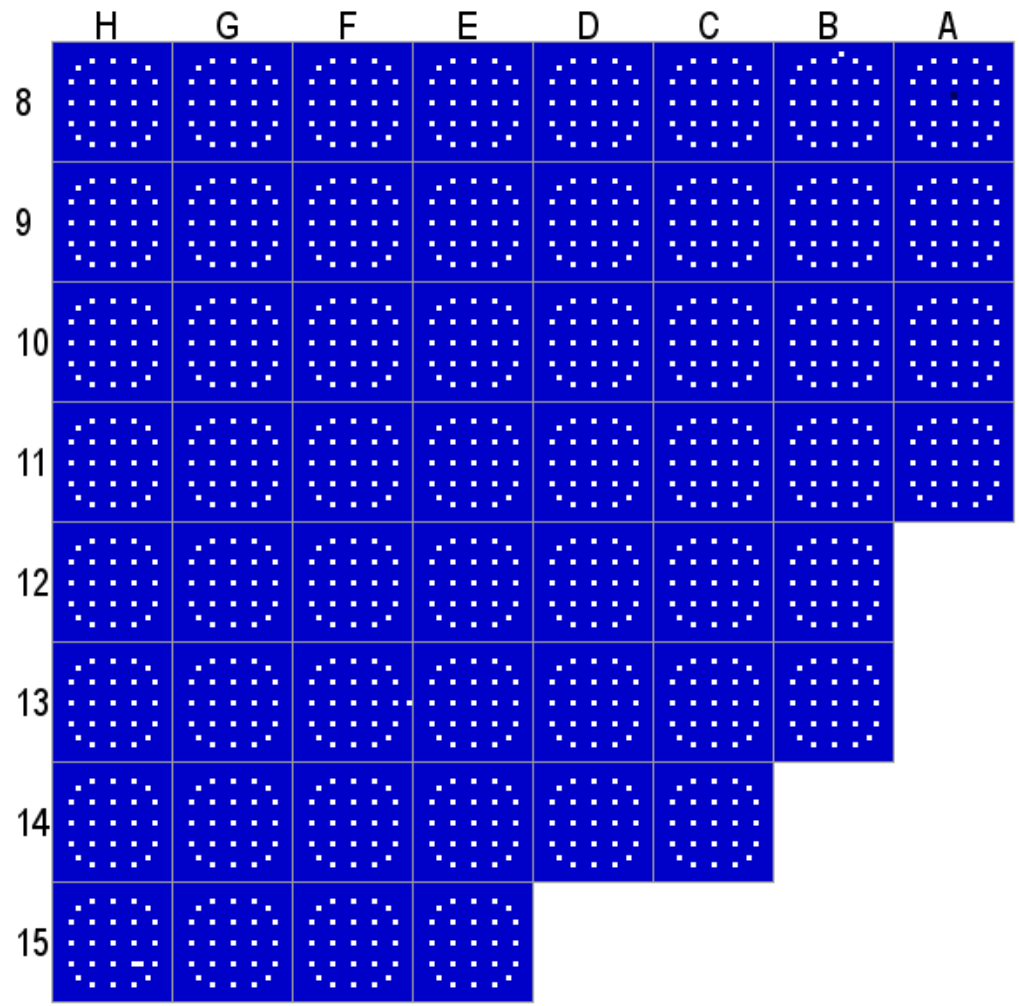

$1.5 e+03-$
$1409.42-1315.57-$
$1221.73-$
$1127.89-$
$1034.05-$
$940-$
$846-$
$753-$
$659-$

Fig. A.2.1. Cycle 2 maximum centerline fuel temperature $(\mathrm{K})-0.00 \mathrm{GWd} / \mathrm{MT}$.

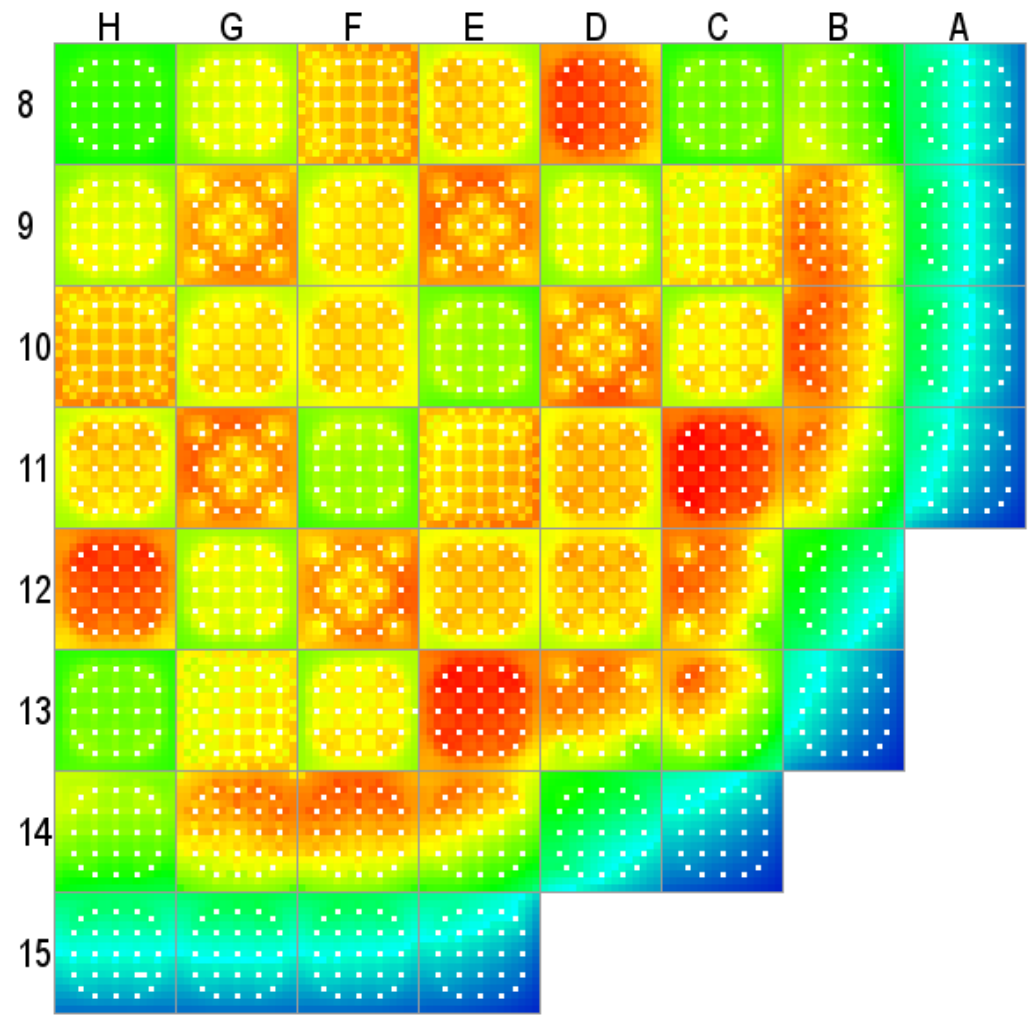

$1.5 e+03-$
1409.42
1315.57
$1221.73-$
1127.89
1034.05
$940-$
$846-$
$753-$
$659-$

Fig. A.2.2. Cycle 2 maximum centerline fuel temperature $(\mathrm{K})-0.14$ GWd/MT. 

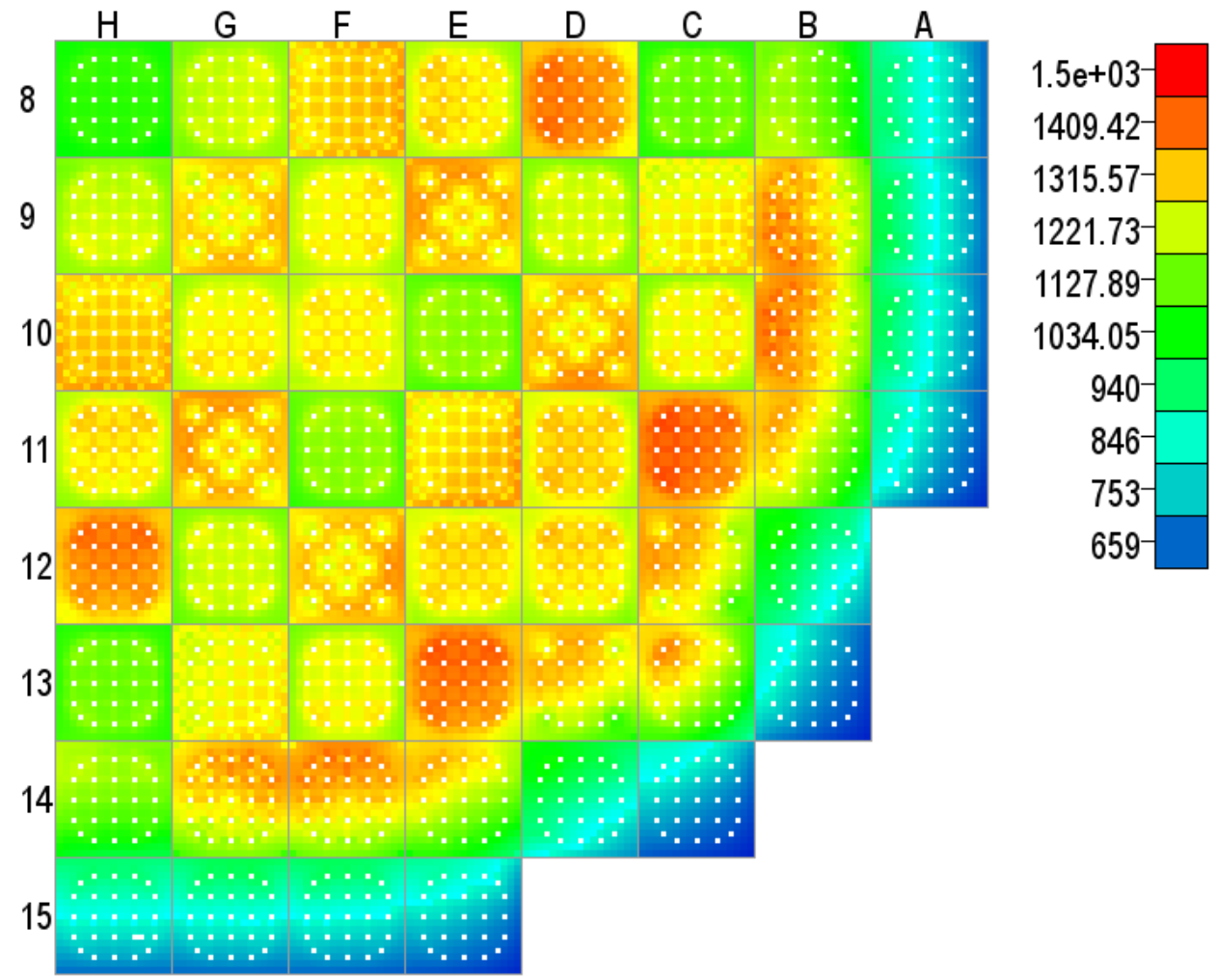

Fig. A.2.3. Cycle 2 maximum centerline fuel temperature $(\mathrm{K})-0.52 \mathrm{GWd} / \mathrm{MT}$.

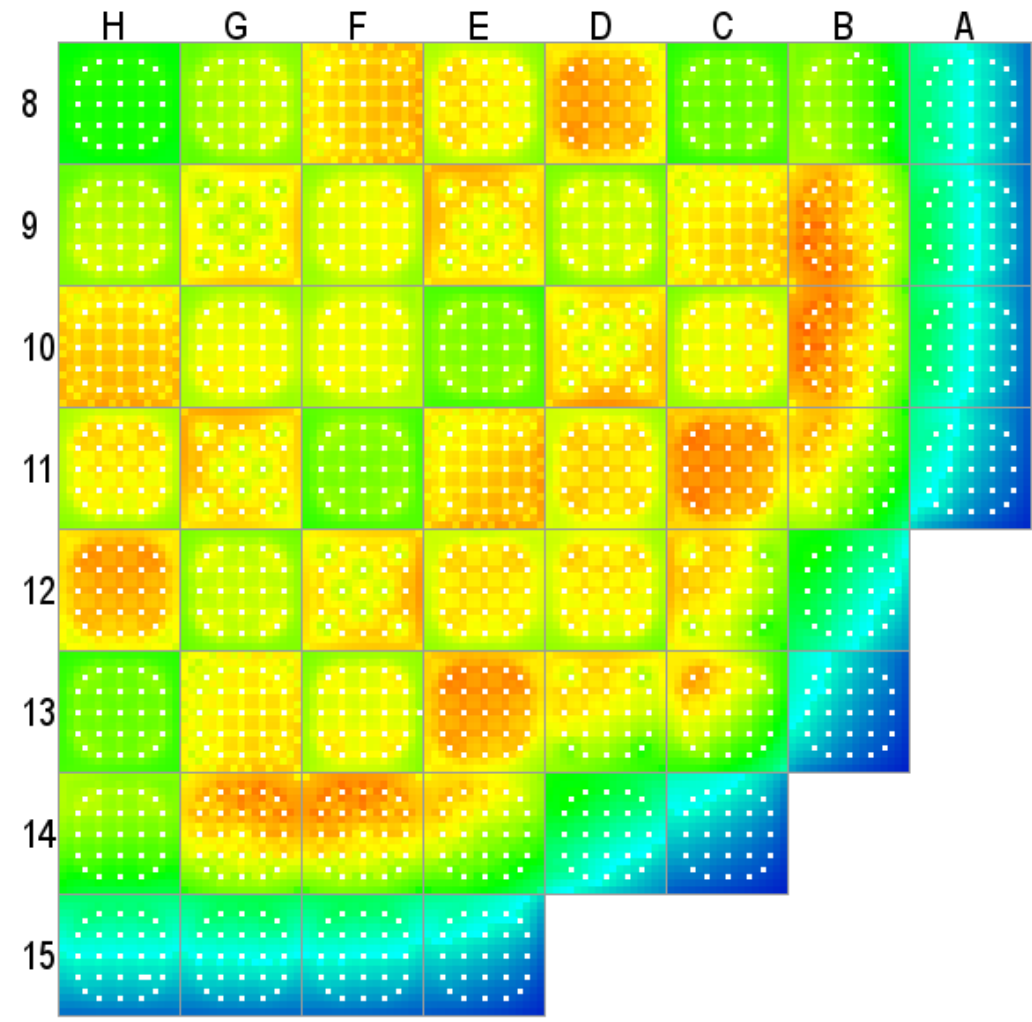

$1.5 e+03-$
1409.42
1315.57
1221.73
1127.89
1034.05
$940-$
846
$753-$
$659-$

Fig. A.2.4. Cycle 2 maximum centerline fuel temperature $(\mathrm{K})-1.00 \mathrm{GWd} / \mathrm{MT}$. 


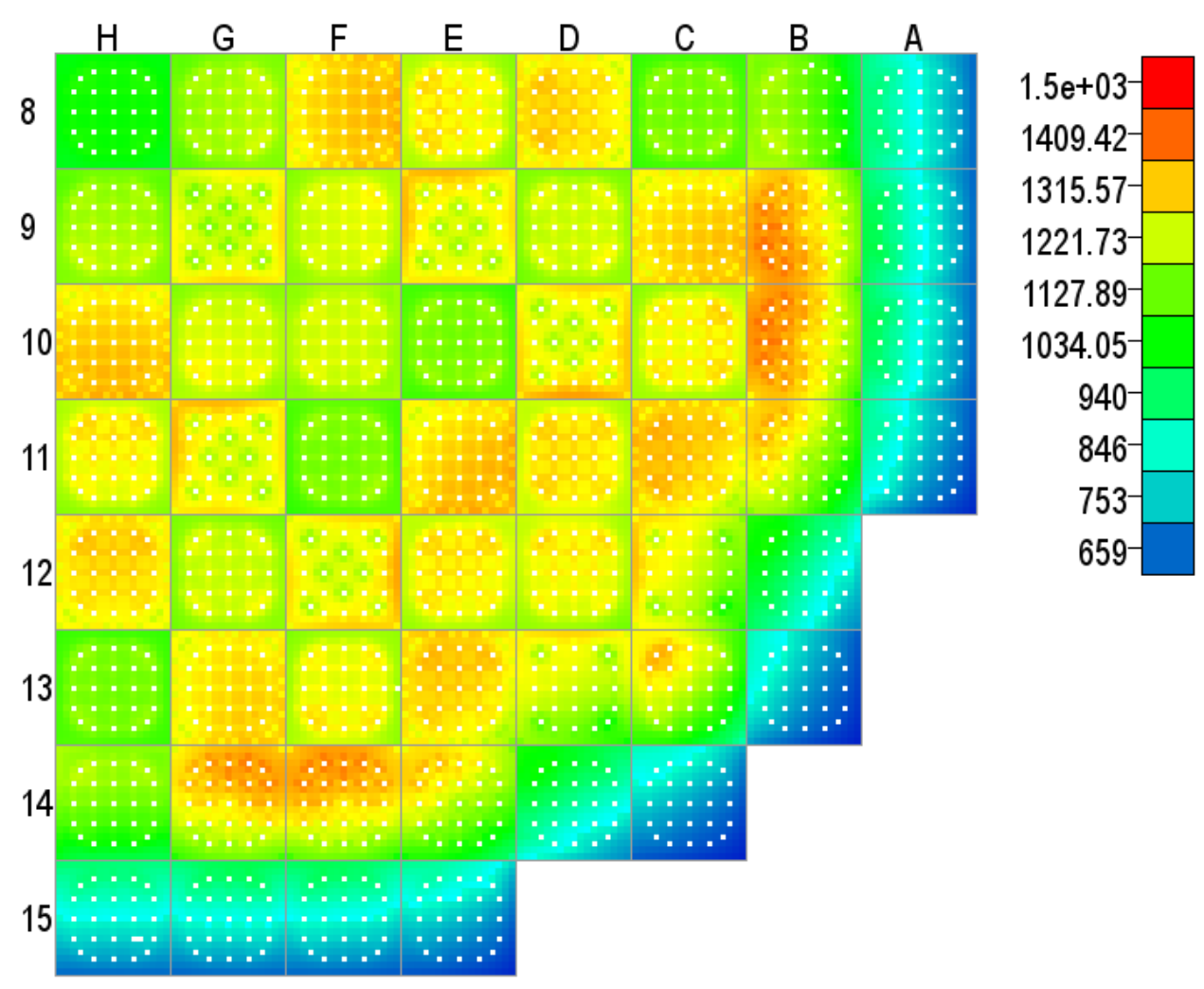

Fig. A.2.5. Cycle 2 maximum centerline fuel temperature $(\mathrm{K})-1.48 \mathrm{GWd} / \mathrm{MT}$.

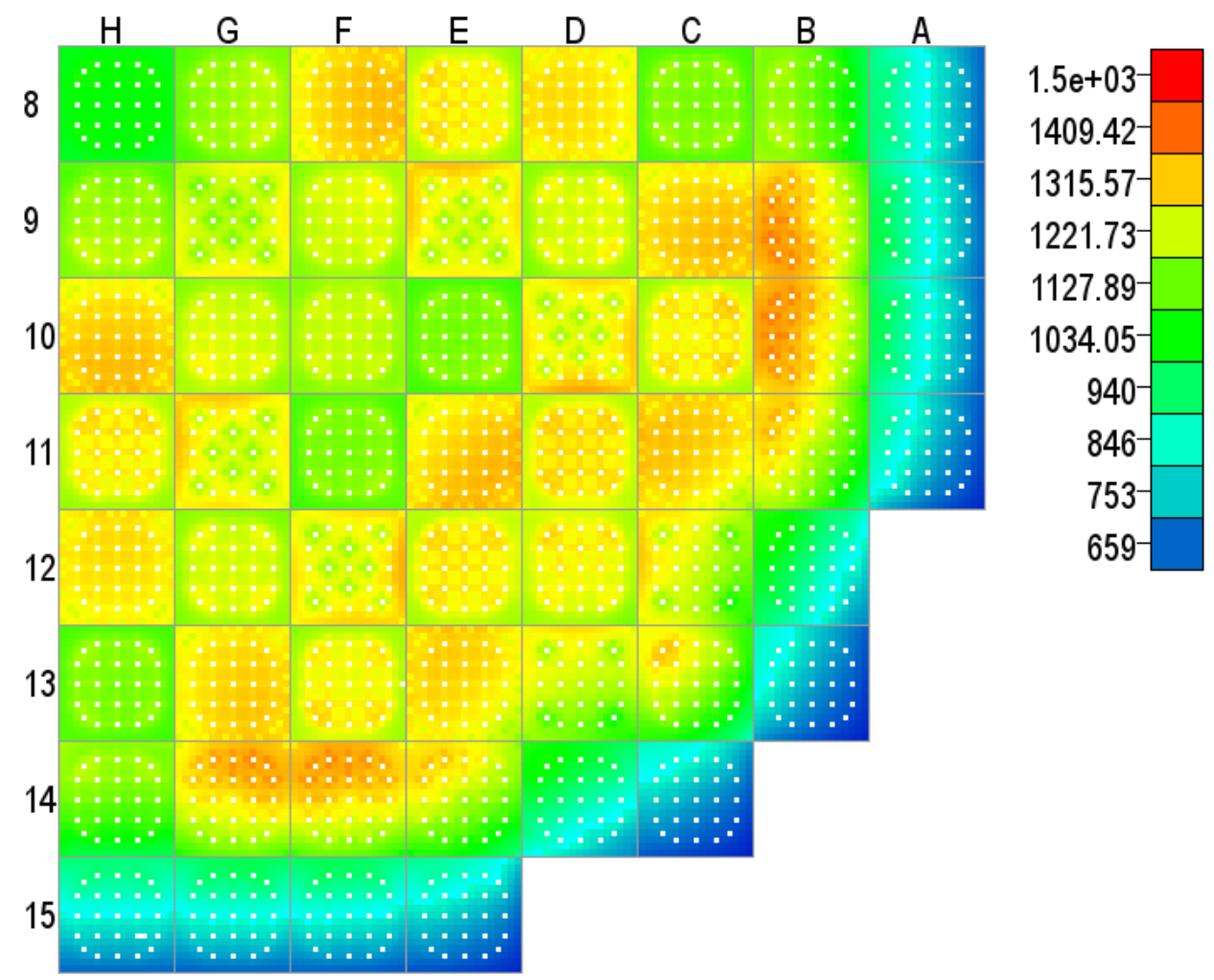

Fig. A.2.6. Cycle 2 maximum centerline fuel temperature $(\mathrm{K})-2.07$ GWd/MT. 

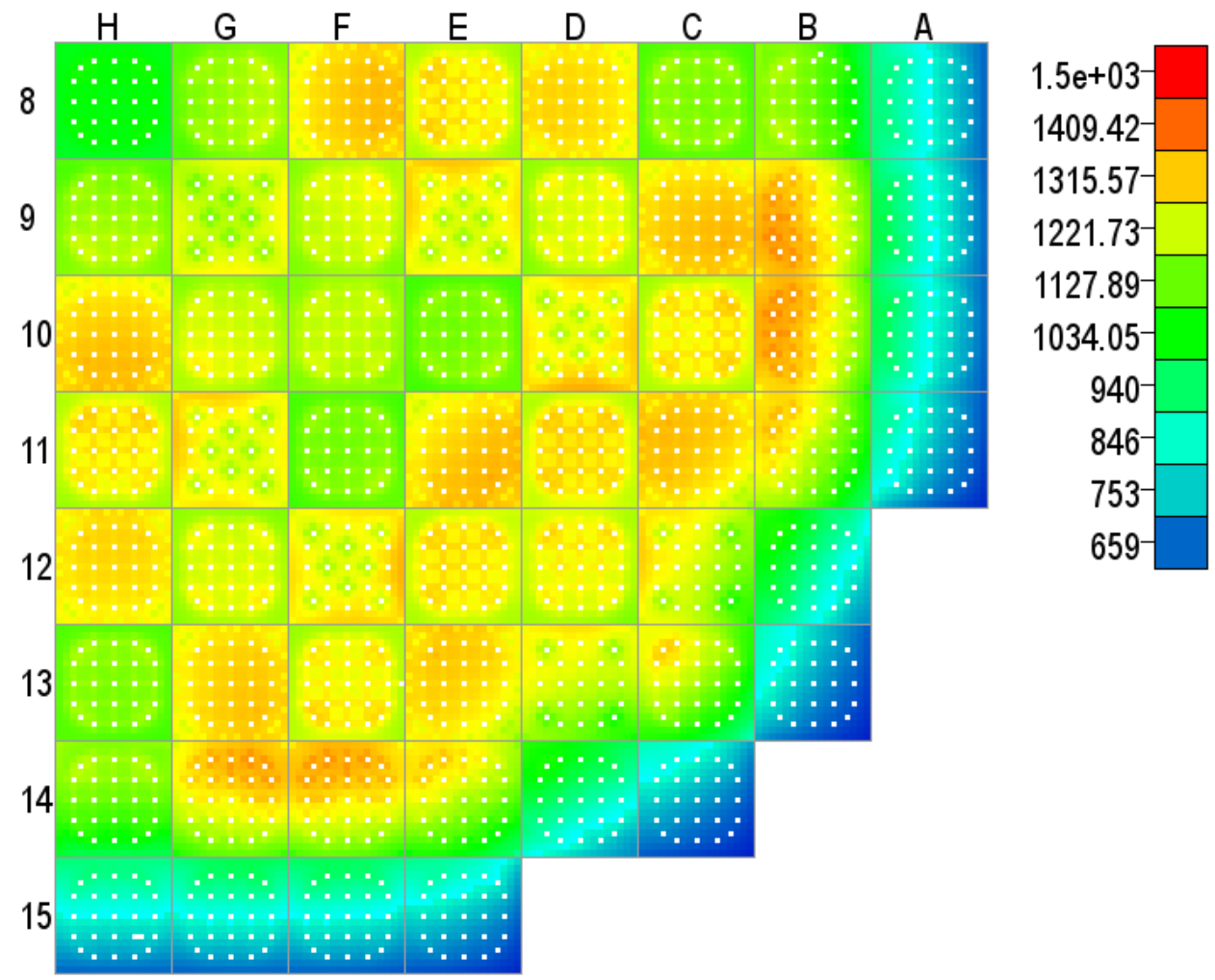

Fig. A.2.7. Cycle 2 maximum centerline fuel temperature $(\mathrm{K})-2.48 \mathrm{GWd} / \mathrm{MT}$.
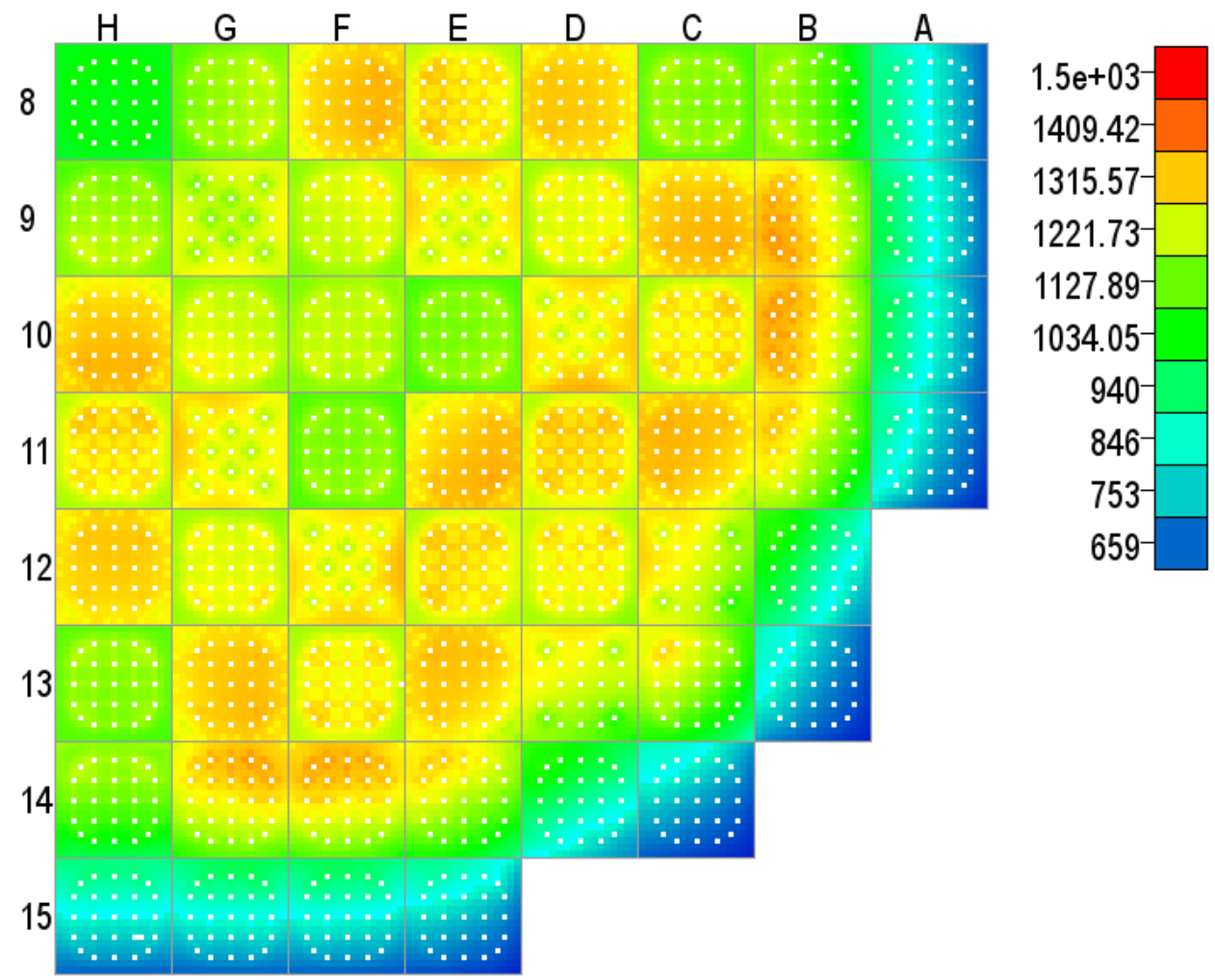

Fig. A.2.8. Cycle 2 maximum centerline fuel temperature $(\mathrm{K})-3.13 \mathrm{GWd} / \mathrm{MT}$. 

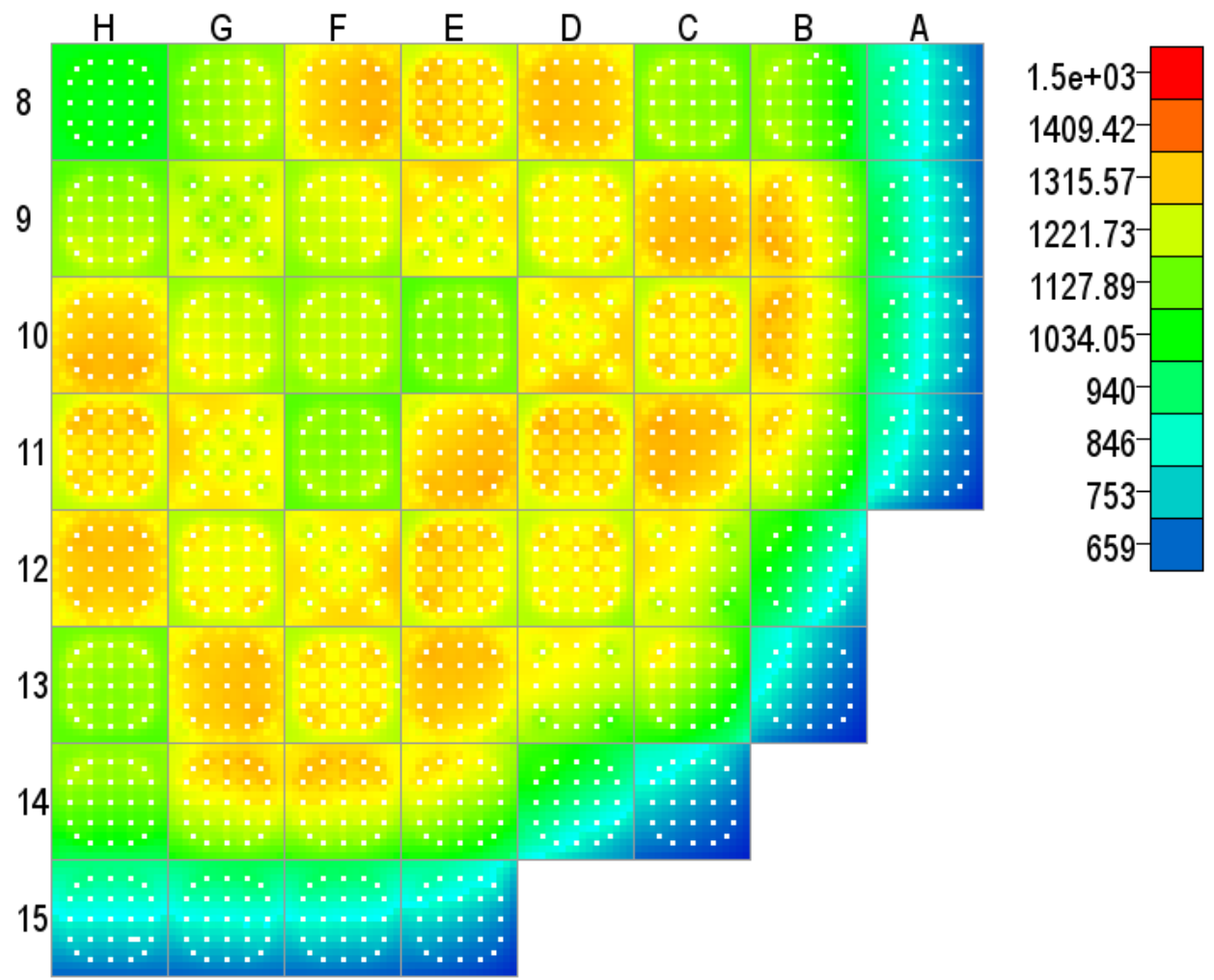

Fig. A.2.9. Cycle 2 maximum centerline fuel temperature $(\mathrm{K})-4.02 \mathrm{GWd} / \mathrm{MT}$.

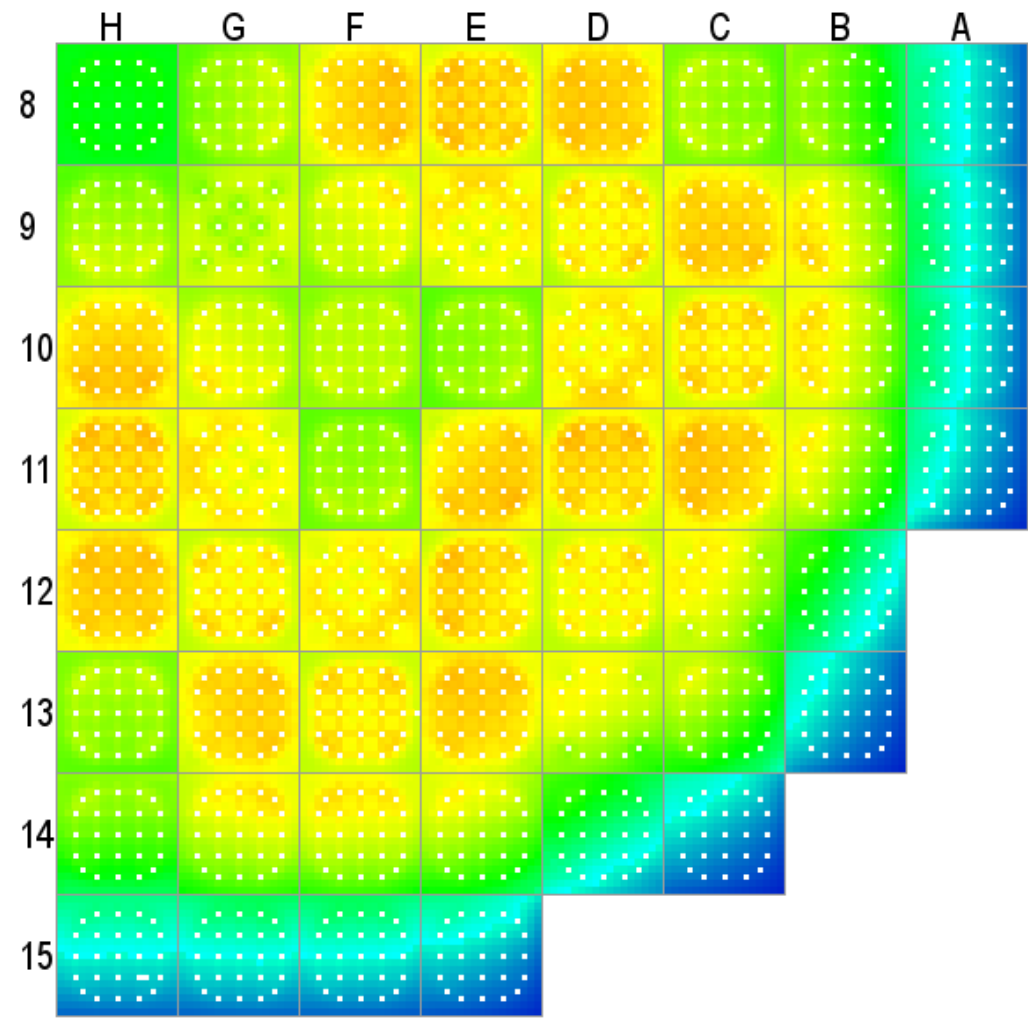

$1.5 e+03-$
1409.42
1315.57
$1221.73-$
$1127.89-$
1034.05
$940-$
$846-$
$753-$
$659-$

Fig. A.2.10. Cycle 2 maximum centerline fuel temperature $(\mathrm{K})-5.53 \mathrm{GWd} / \mathrm{MT}$. 

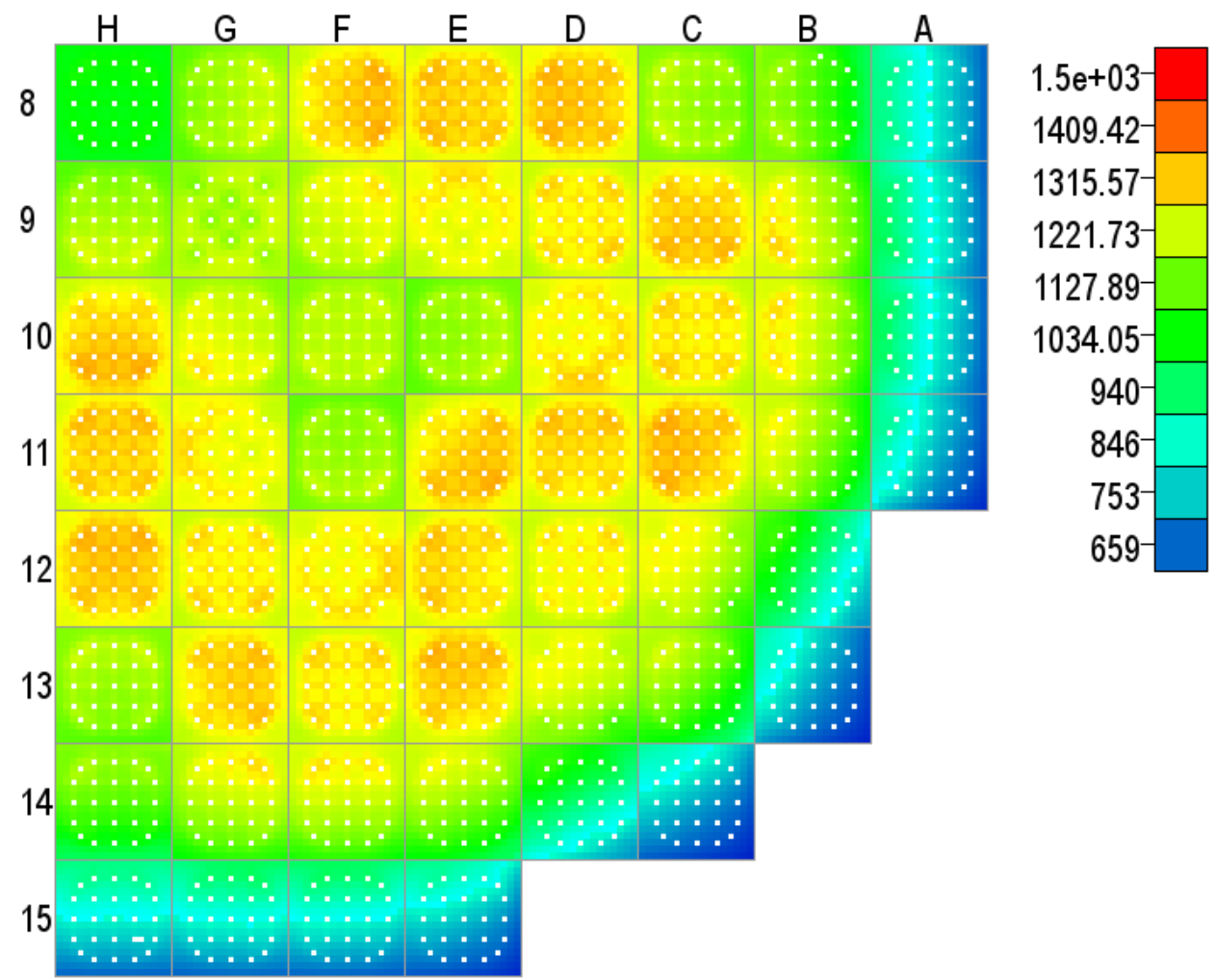

Fig. A.2.11. Cycle 2 maximum centerline fuel temperature $(\mathrm{K})-6.53 \mathrm{GWd} / \mathrm{MT}$.

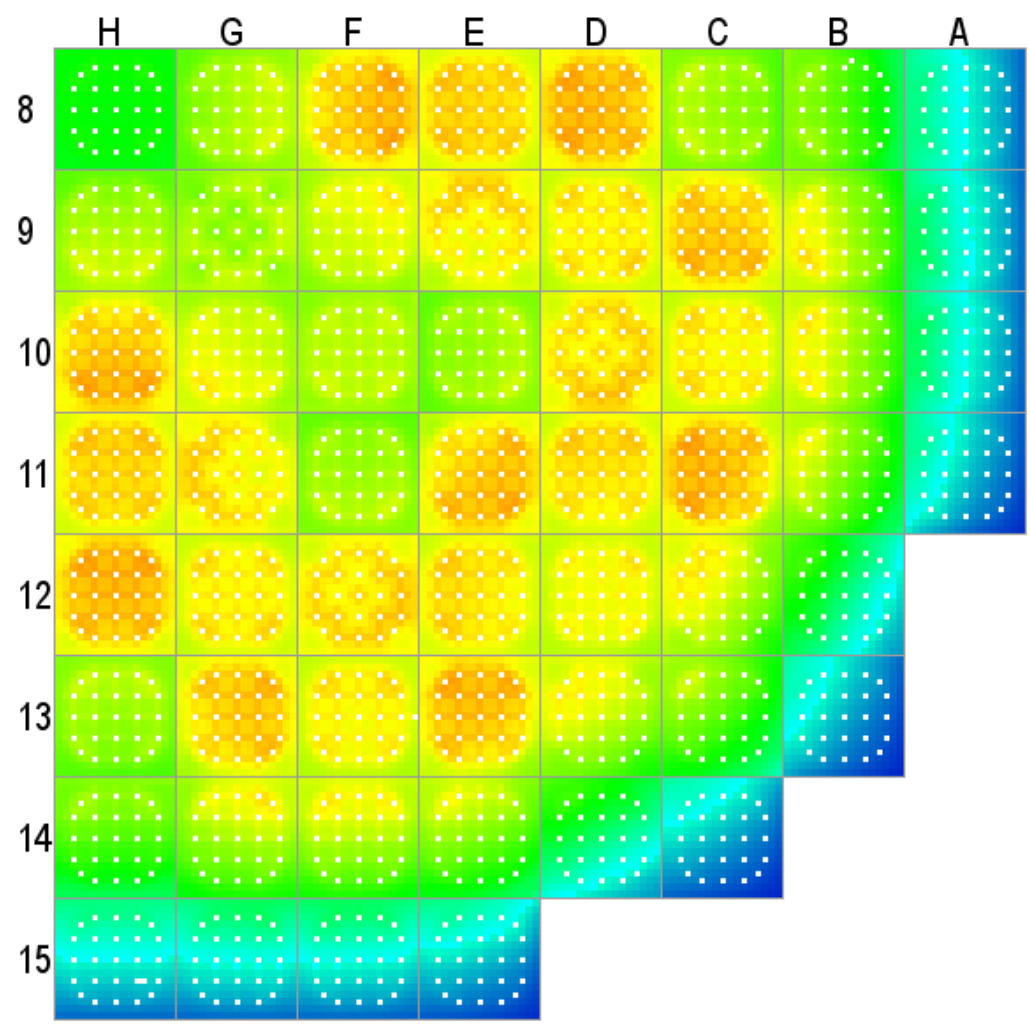

$1.5 e+03$ 1409.42 1315.57 1221.73 1127.89 1034.05

$$
940
$$

846 753 $659-$

Fig. A.2.12. Cycle 2 maximum centerline fuel temperature $(\mathrm{K})-8.22 \mathrm{GWd} / \mathrm{MT}$. 


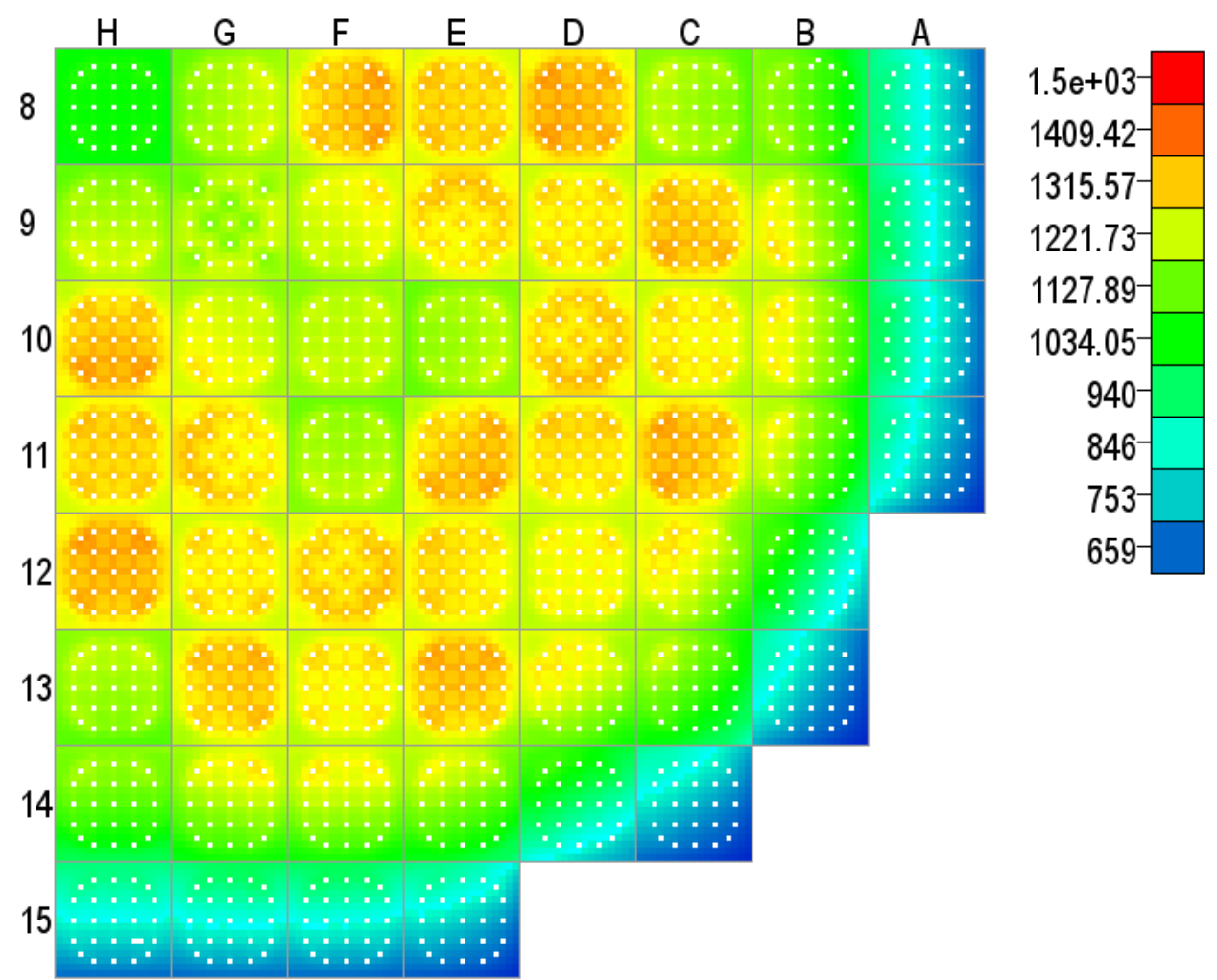

Fig. A.2.13. Cycle 2 maximum centerline fuel temperature (K) - 9.29 GWd/MT.

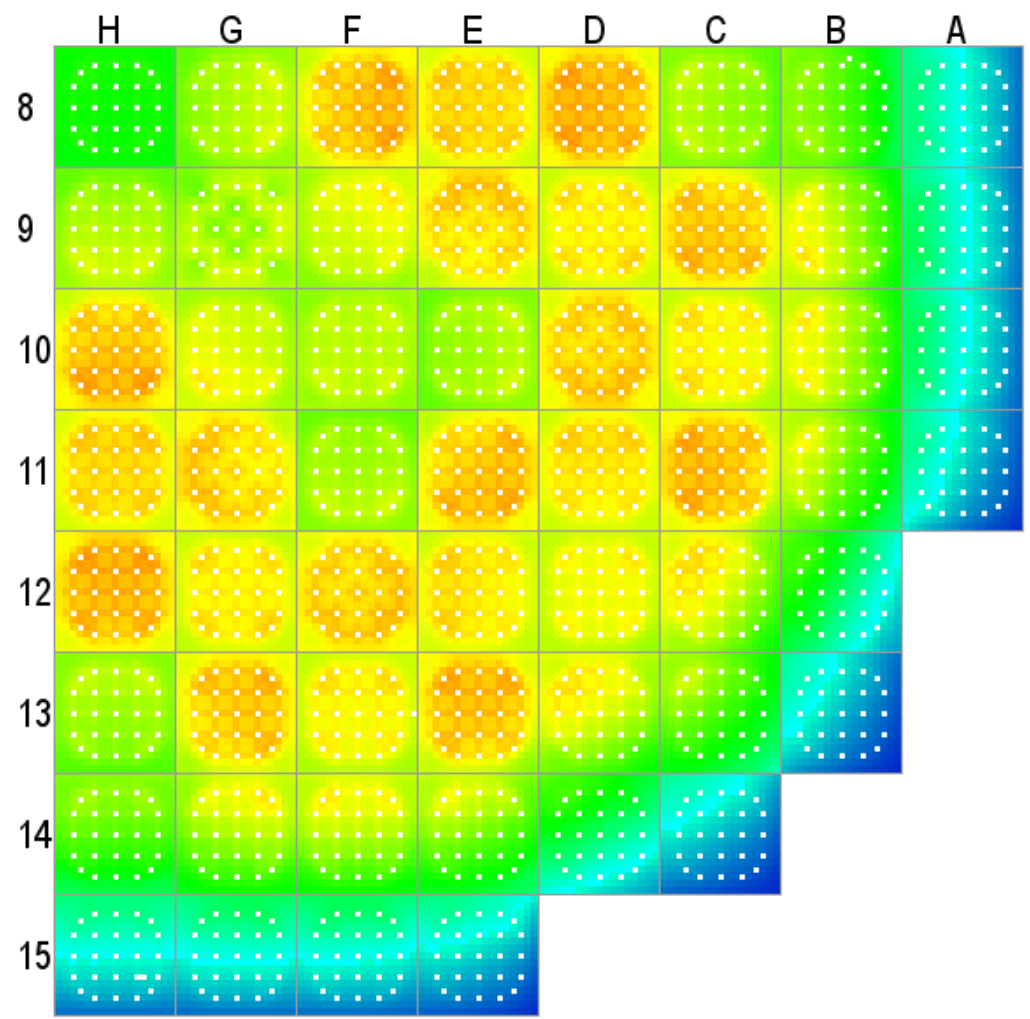

$1.5 e+03$

$1409.42-$

1315.57

$1221.73-$

1127.89

1034.05

940

$846-$

753

659

Fig. A.2.14. Cycle 2 maximum centerline fuel temperature $(\mathrm{K})-10.36 \mathrm{GWd} / \mathrm{MT}$. 

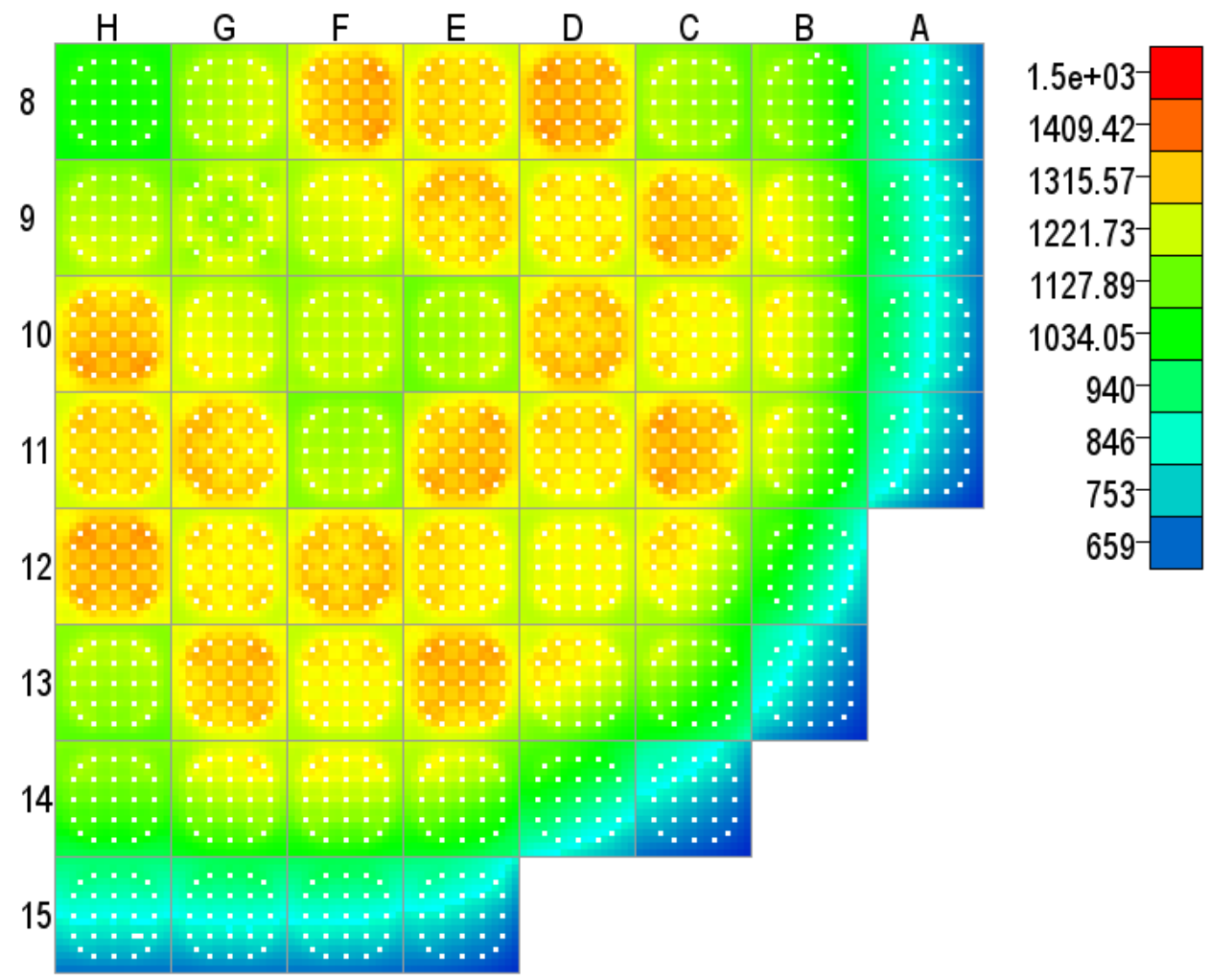

Fig. A.2.15. Cycle 2 maximum centerline fuel temperature $(\mathrm{K})-11.43$ GWd/MT.
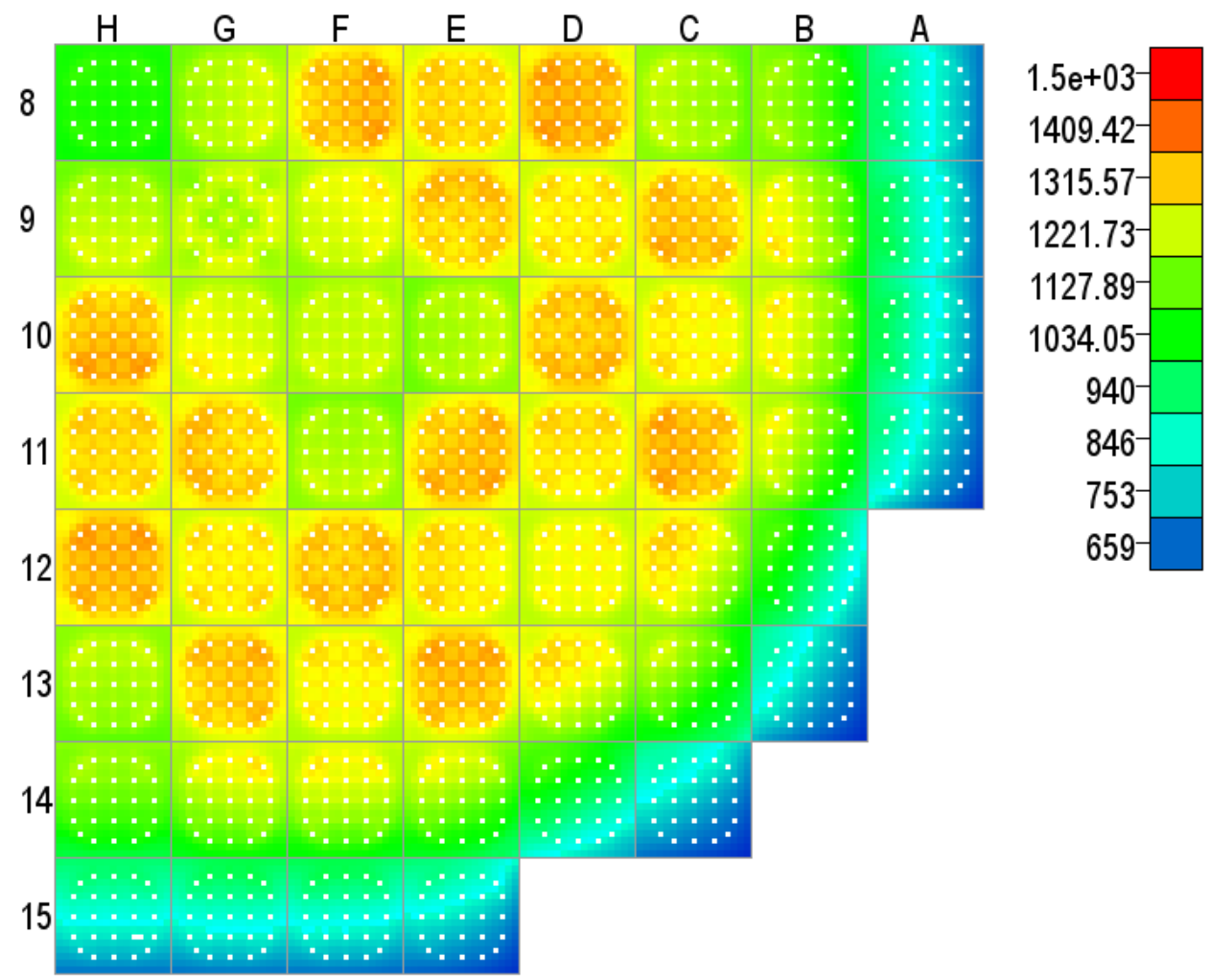

Fig. A.2.16. Cycle 2 maximum centerline fuel temperature (K) - 12.54 GWd/MT. 


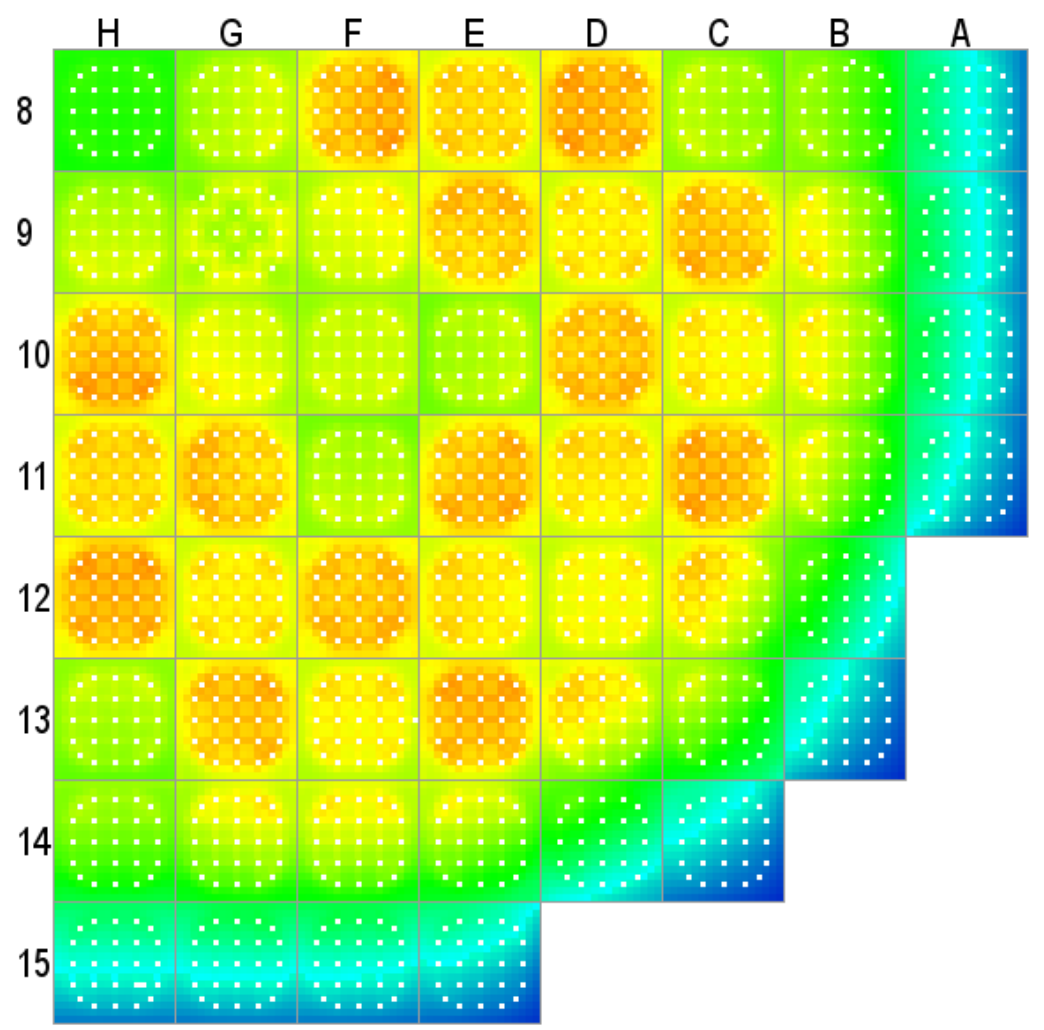

$1.5 e+03-$
1409.42
$1315.57-$
$1221.73-$
1127.89
1034.05
$940-$
$846-$
$753-$
$659-$

Fig. A.2.17. Cycle 2 maximum centerline fuel temperature $(\mathrm{K})-13.54 \mathrm{GWd} / \mathrm{MT}$.
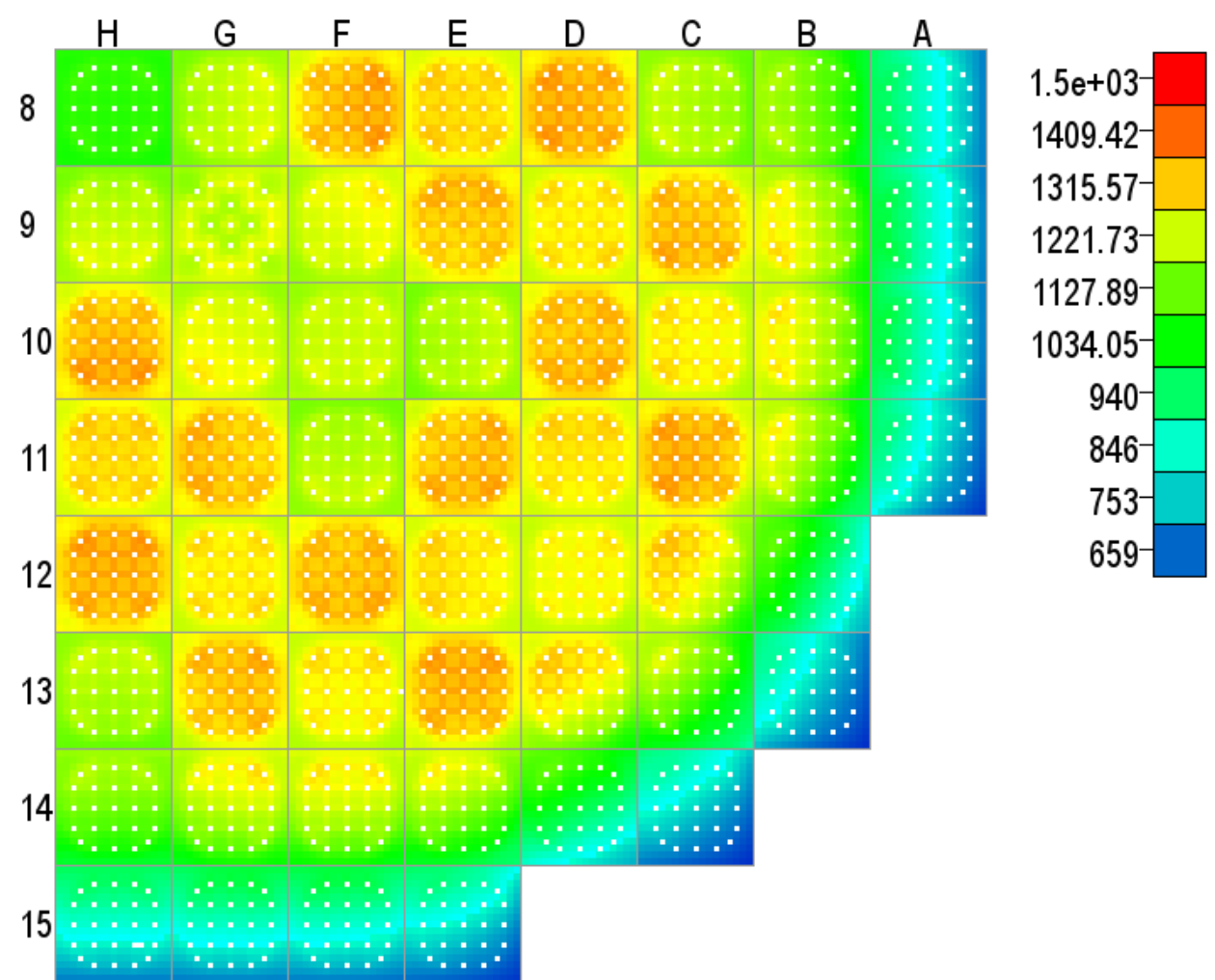

Fig. A.2.18. Cycle 2 maximum centerline fuel temperature $(\mathrm{K})-14.62 \mathrm{GWd} / \mathrm{MT}$. 

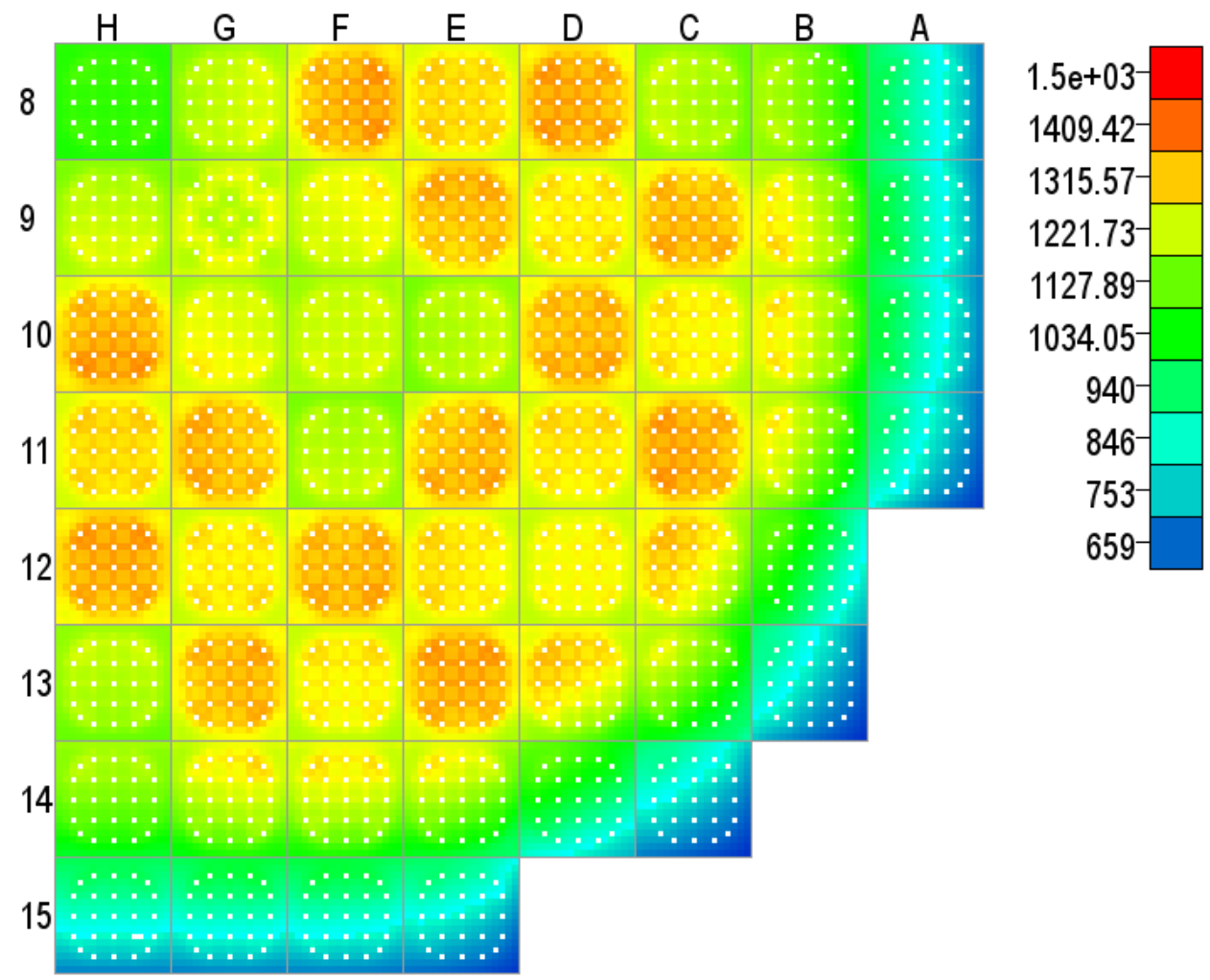

Fig. A.2.19. Cycle 2 maximum centerline fuel temperature $(\mathrm{K})-15.76 \mathrm{GWd} / \mathrm{MT}$.

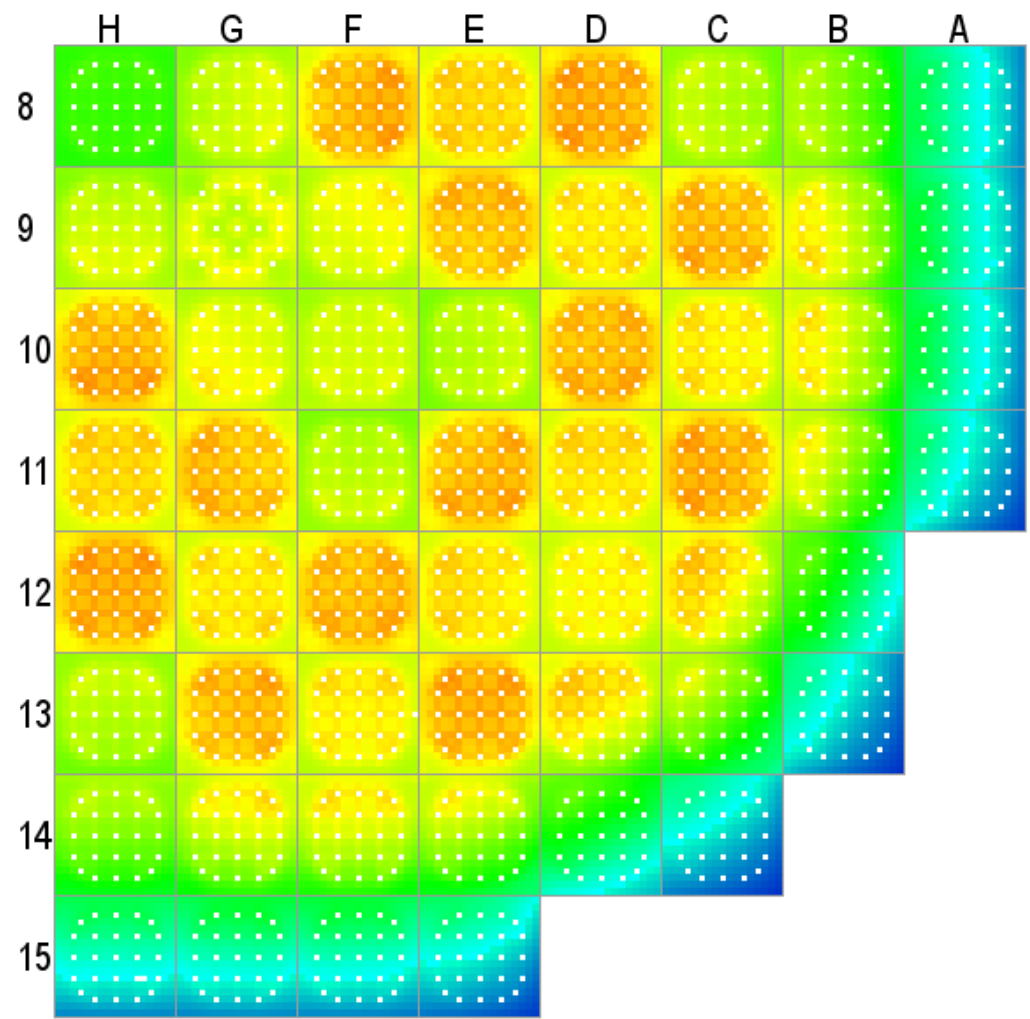

Fig. A.2.20. Cycle 2 maximum centerline fuel temperature $(\mathrm{K})-16.32 \mathrm{GWd} / \mathrm{MT}$. 

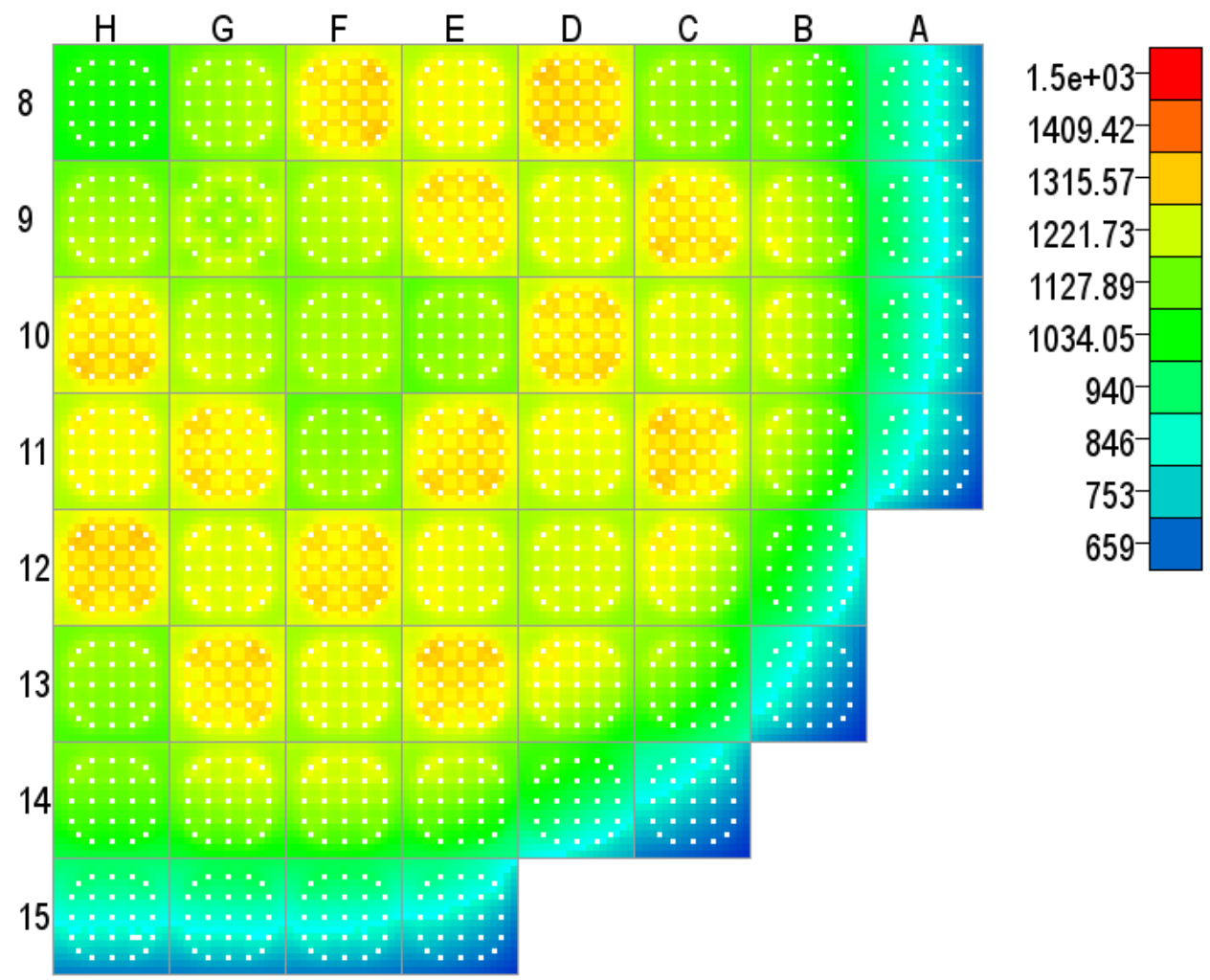

Fig. A.2.21. Cycle 2 maximum centerline fuel temperature $(\mathrm{K})-16.90 \mathrm{GWd} / \mathrm{MT}$.

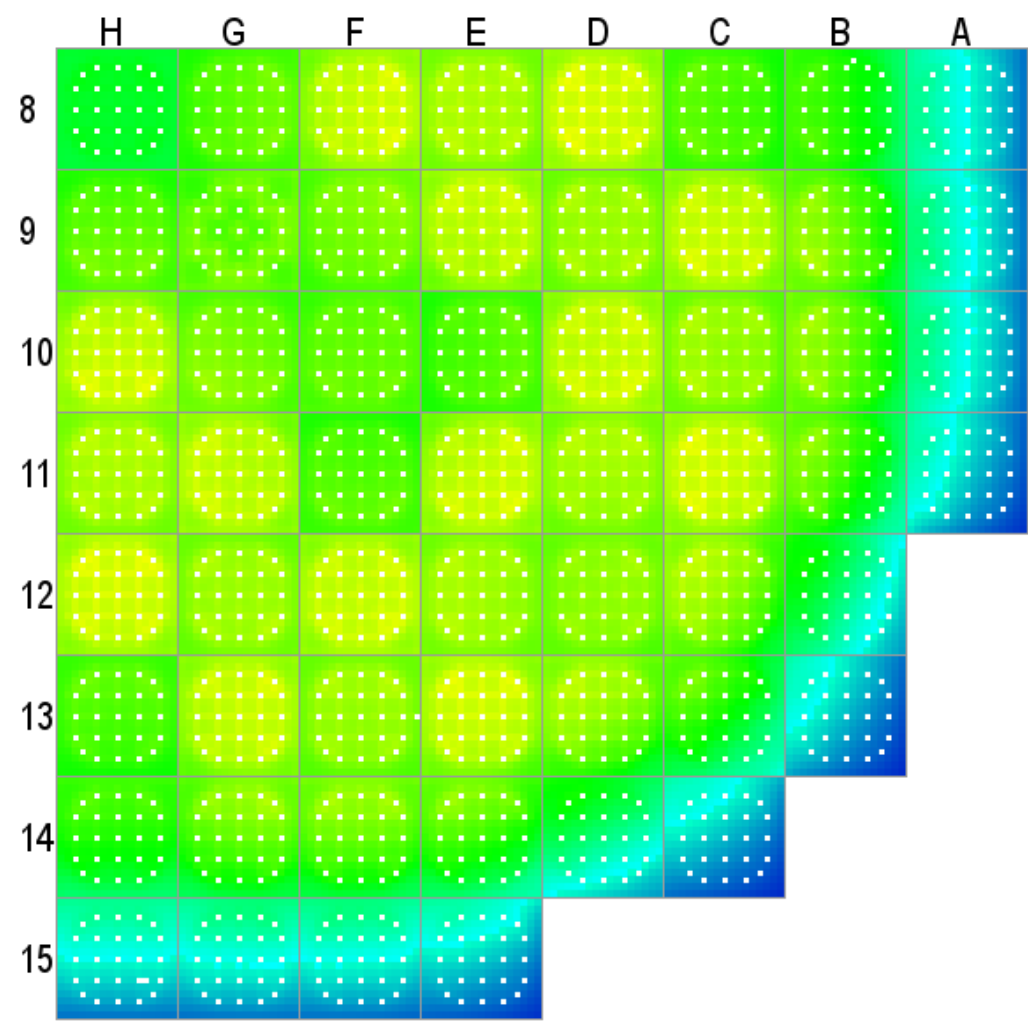

$1.5 \mathrm{e}+03$

1409.42

1315.57

1221.73

1127.89

1034.05

$940^{-}$

846

753

659

Fig. A.2.22. Cycle 2 maximum centerline fuel temperature $(\mathrm{K})-17.48 \mathrm{GWd} / \mathrm{MT}$. 


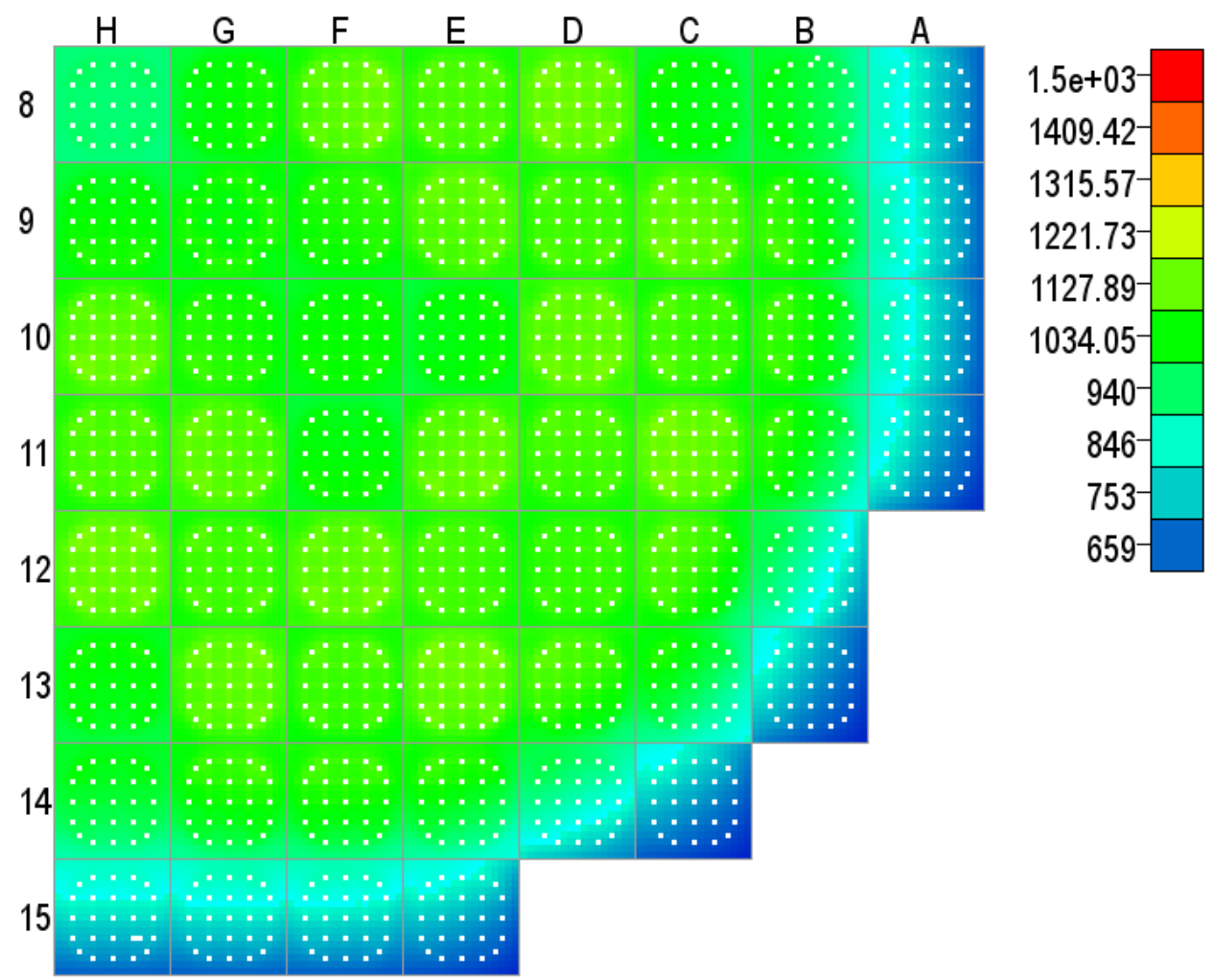

Fig. A.2.23. Cycle 2 maximum centerline fuel temperature $(\mathrm{K})-18.06 \mathrm{GWd} / \mathrm{MT}$.

\section{A.2.2. Minimum Gap Thickness}

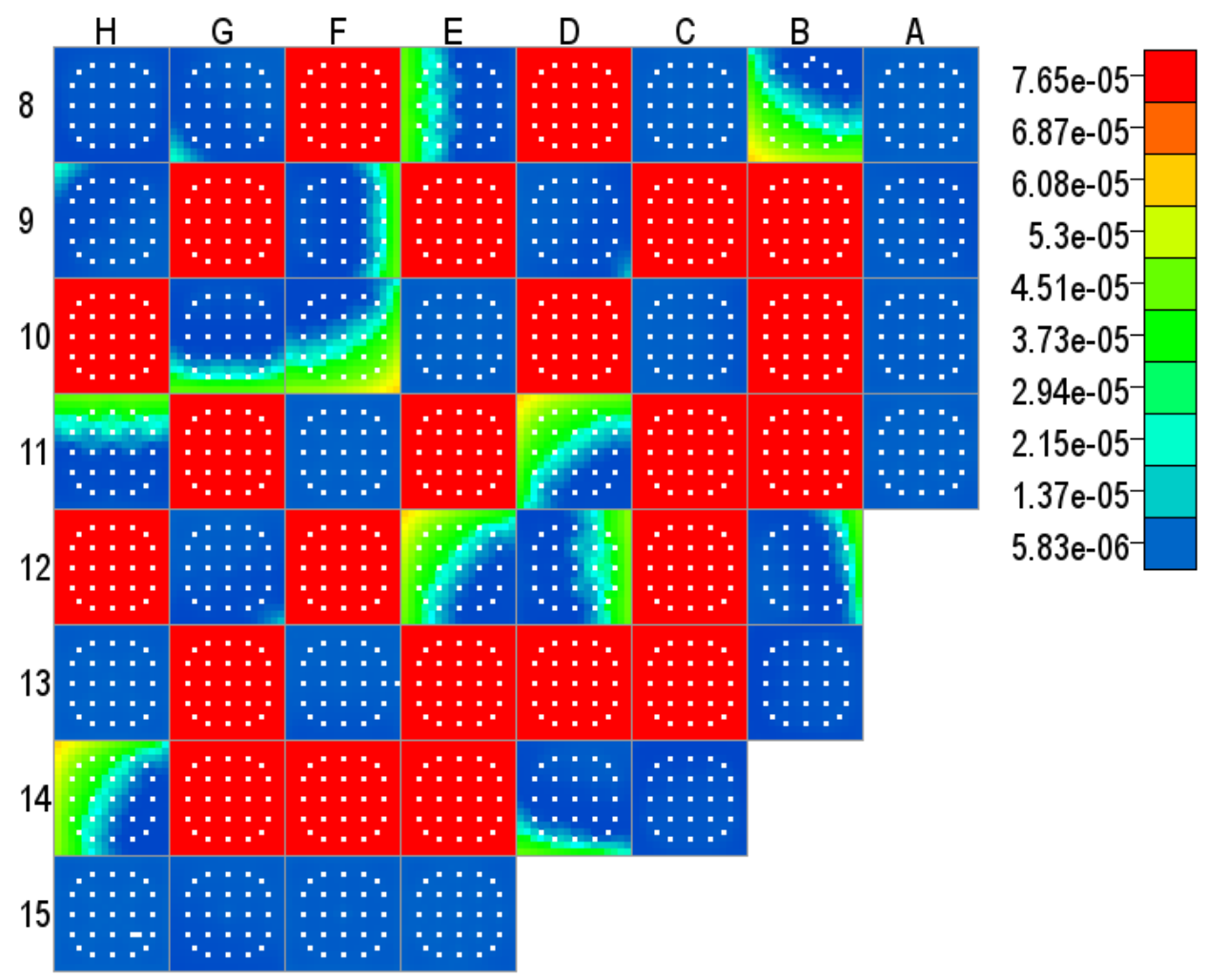

Fig. A.2.24. Cycle 2 minimum gap thickness (m) - 0.00 GWd/MT. 


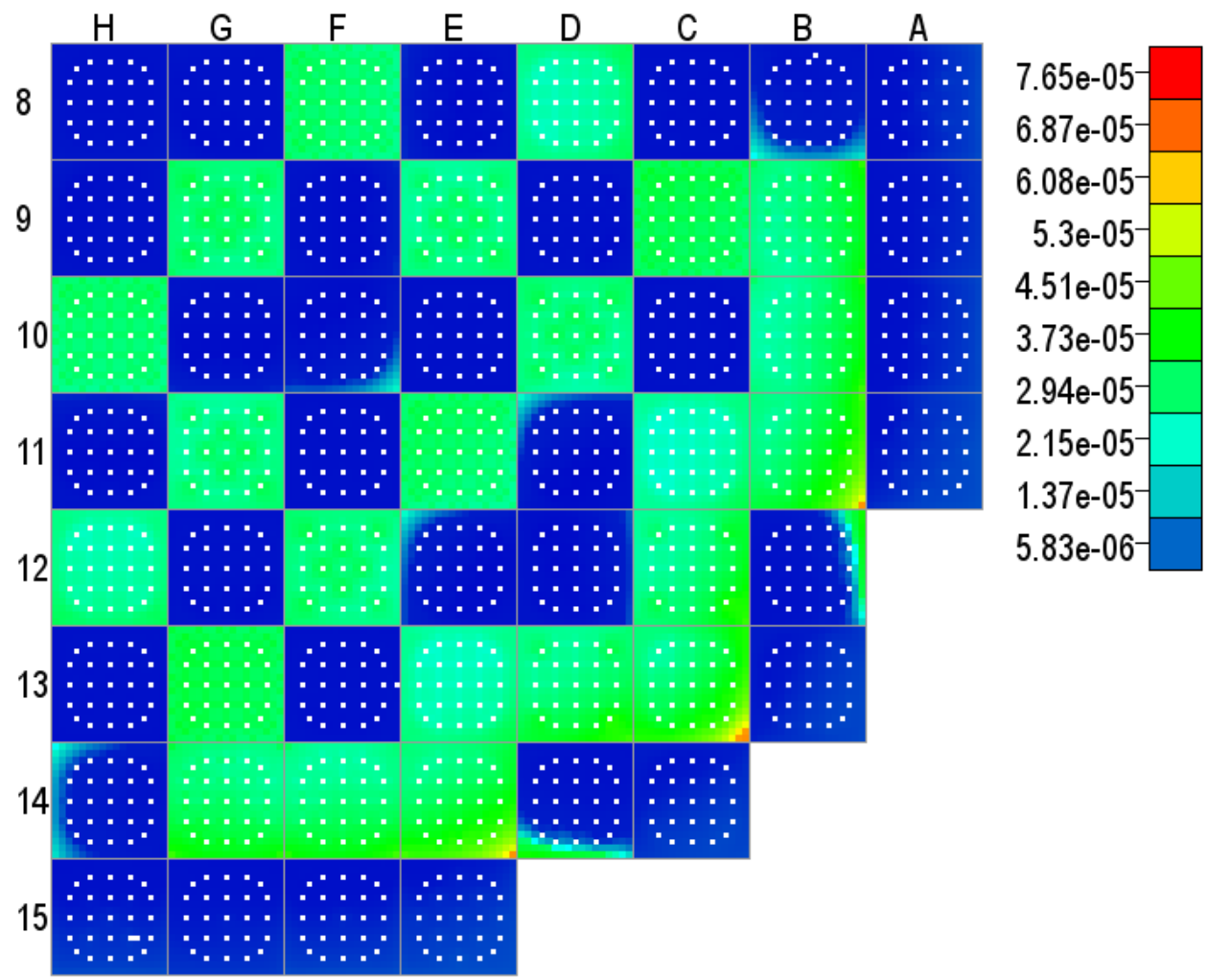

Fig. A.2.25. Cycle 2 minimum gap thickness $(\mathrm{m})-0.14 \mathrm{GWd} / \mathrm{MT}$.

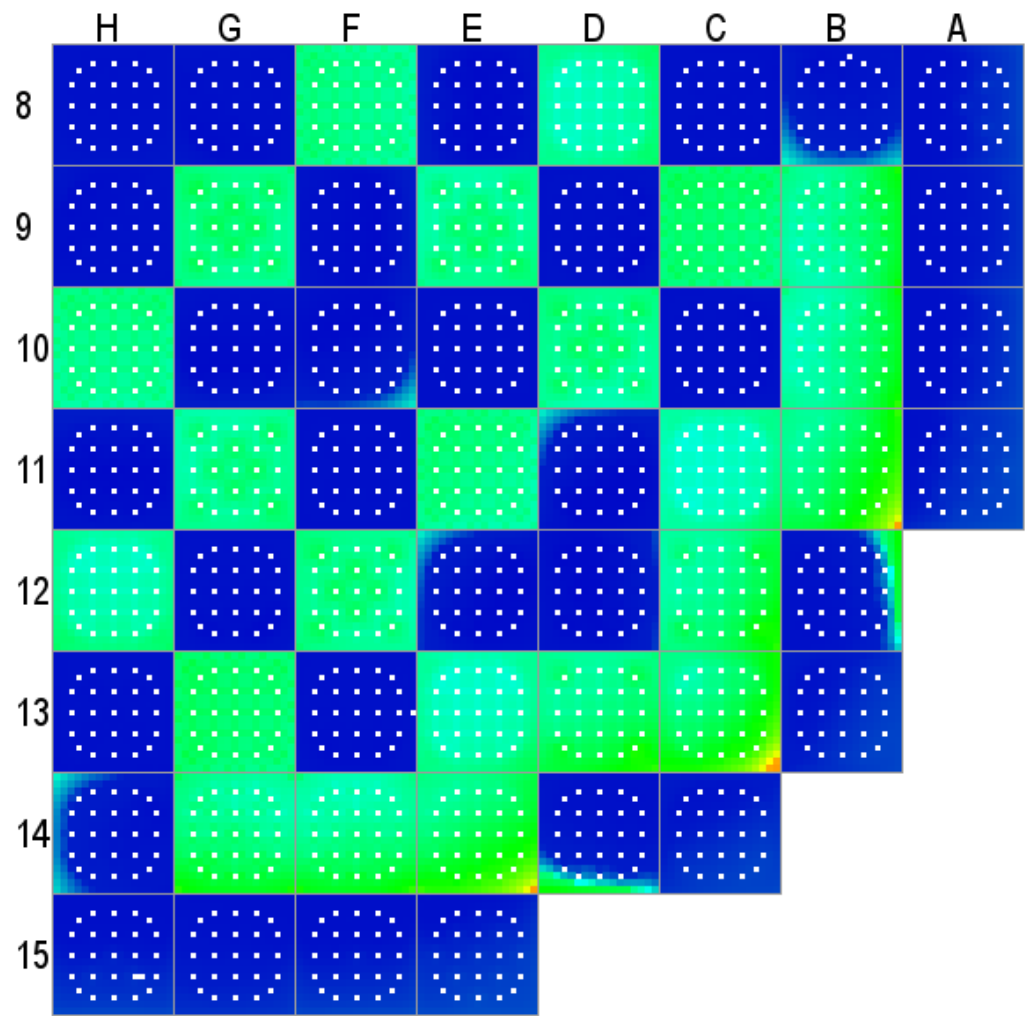

$7.65 \mathrm{e}-05$
$6.87 \mathrm{e}-05$
$6.08 \mathrm{e}-05$
$5.3 \mathrm{e}-05$
$4.51 \mathrm{e}-05$
$3.73 \mathrm{e}-05$
$2.94 \mathrm{e}-05$
$2.15 \mathrm{e}-05$
$1.37 \mathrm{e}-05$
$5.83 \mathrm{e}-06$

Fig. A.2.26. Cycle 2 minimum gap thickness (m) - 0.52 GWd/MT. 


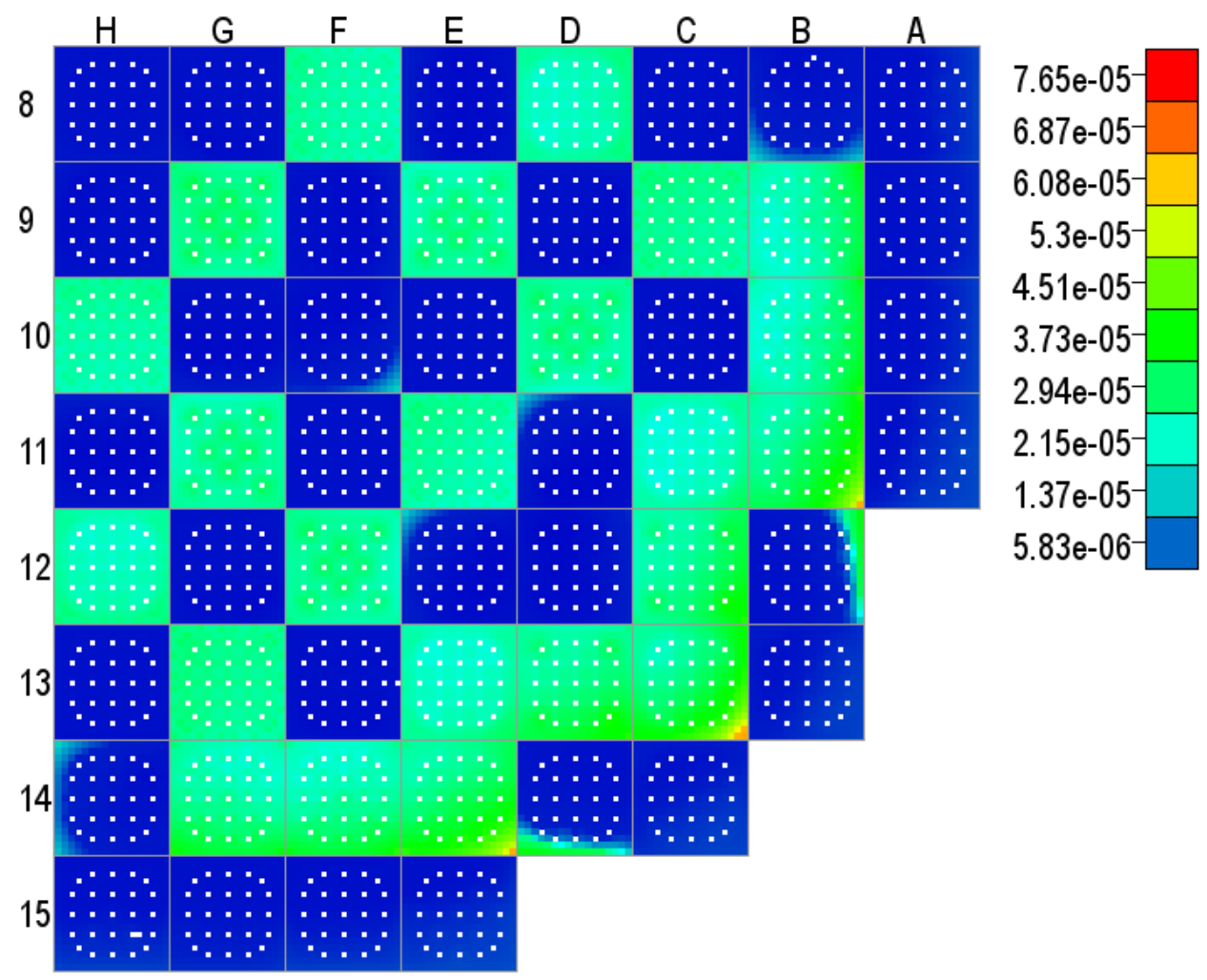

Fig. A.2.27. Cycle 2 minimum gap thickness (m) - 1.00 GWd/MT.

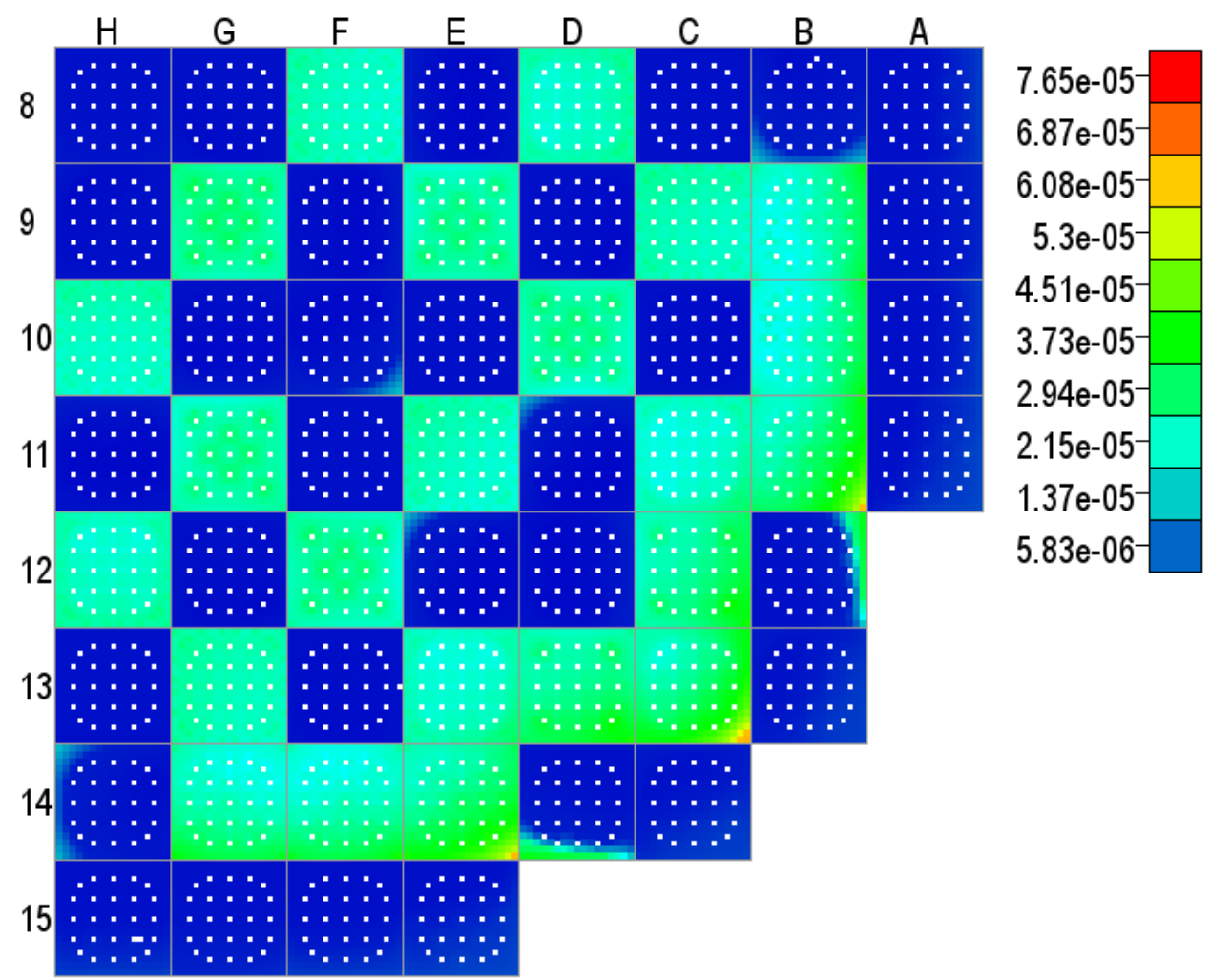

Fig. A.2.28. Cycle 2 minimum gap thickness $(\mathrm{m})-1.48 \mathrm{GWd} / \mathrm{MT}$. 


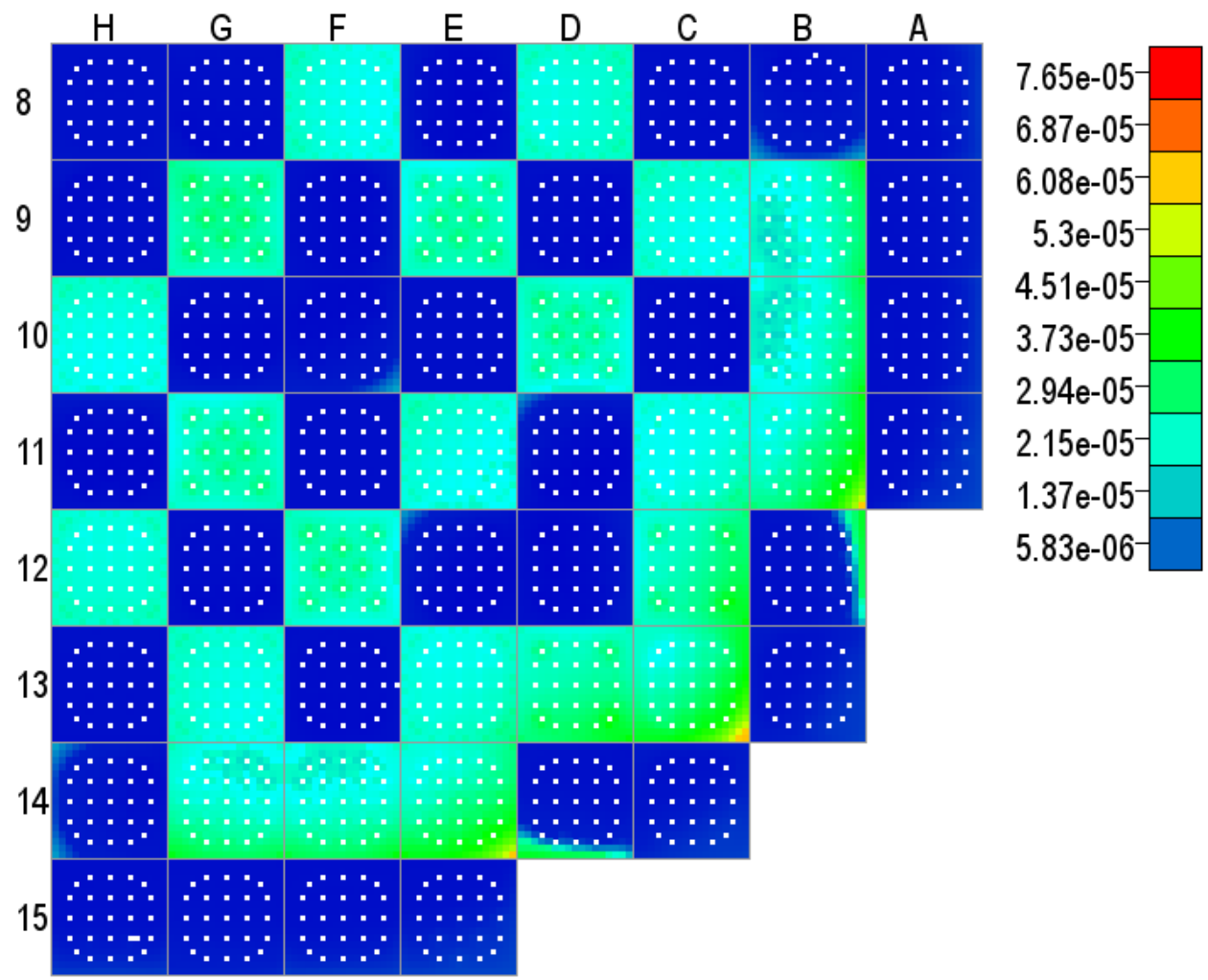

Fig. A.2.29. Cycle 2 minimum gap thickness (m) - 2.07 GWd/MT.

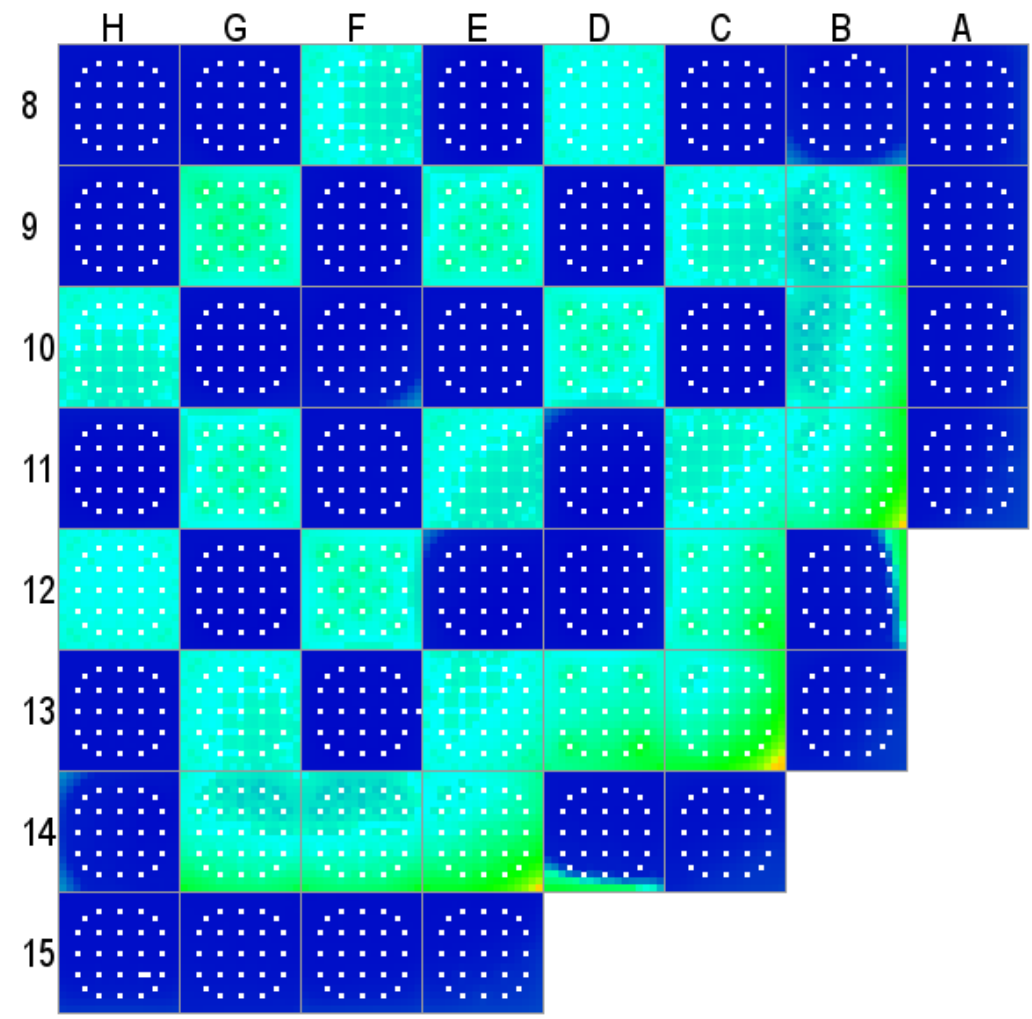

$7.65 \mathrm{e}-05$
$6.87 \mathrm{e}-05$
$6.08 \mathrm{e}-05$
$5.3 \mathrm{e}-05$
$4.51 \mathrm{e}-05$
$3.73 \mathrm{e}-05$
$2.94 \mathrm{e}-05$
$2.15 \mathrm{e}-05$
$1.37 \mathrm{e}-05$
$5.83 \mathrm{e}-05$

Fig. A.2.30. Cycle 2 minimum gap thickness $(\mathrm{m})-2.48 \mathrm{GWd} / \mathrm{MT}$. 


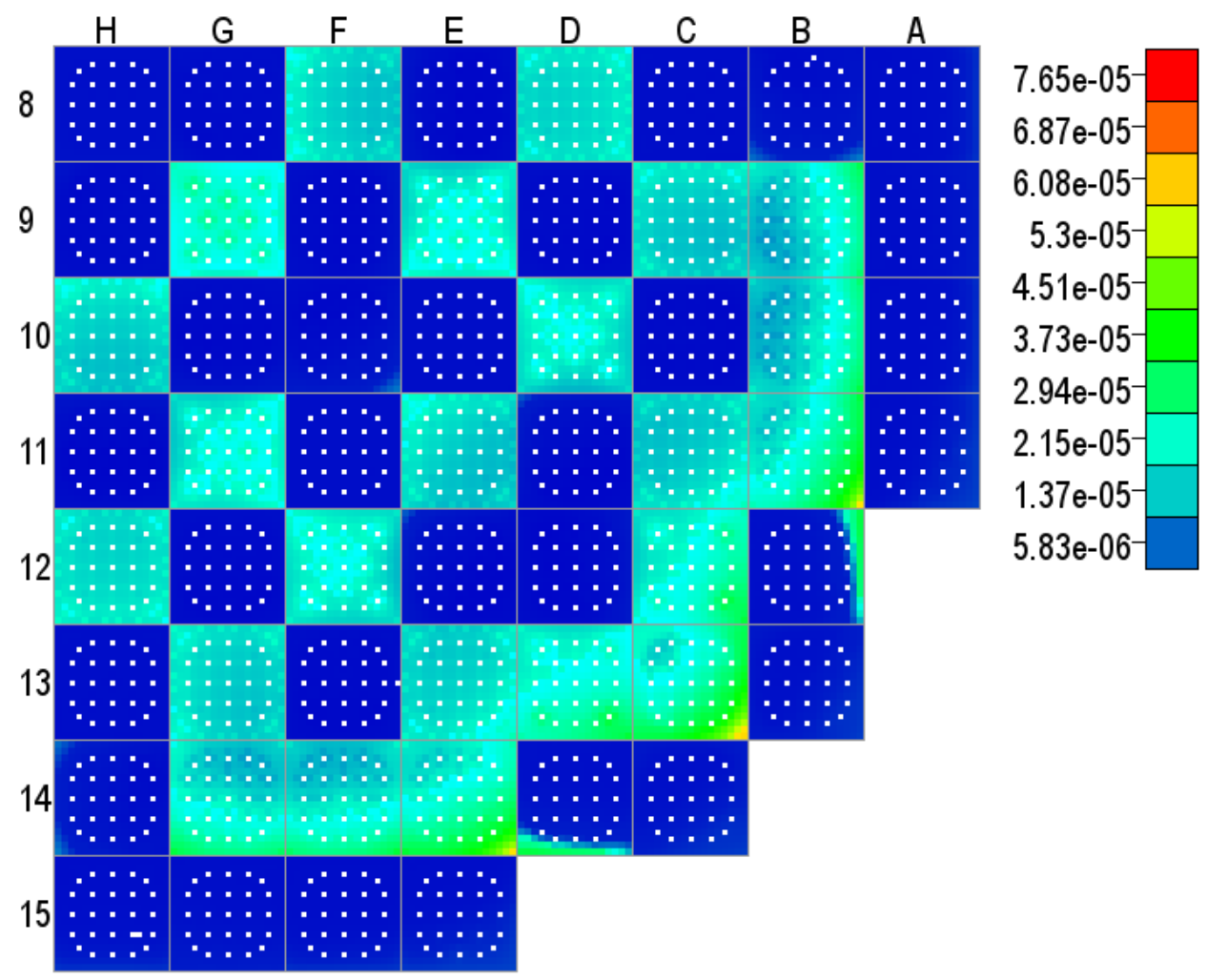

Fig. A.2.31. Cycle 2 minimum gap thickness (m) - 3.13 GWd/MT.

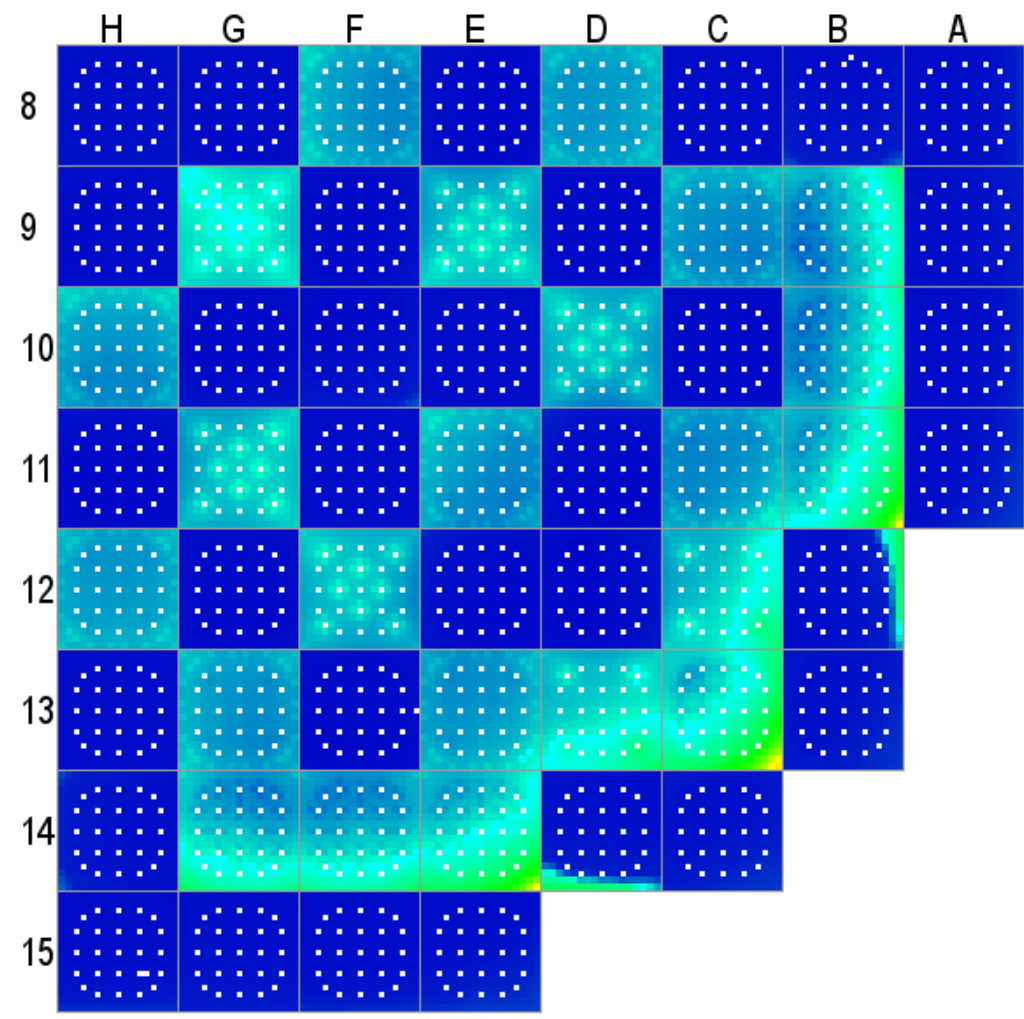

$7.65 \mathrm{e}-05-$
$6.87 \mathrm{e}-05$
$6.08 \mathrm{e}-05$
$5.3 \mathrm{e}-05$
$4.51 \mathrm{e}-05$
$3.73 \mathrm{e}-05$
$2.94 \mathrm{e}-05$
$2.15 \mathrm{e}-05$
$1.37 \mathrm{e}-05$
$5.83 \mathrm{e}-06$

Fig. A.2.32. Cycle 2 minimum gap thickness $(\mathrm{m})-4.02 \mathrm{GWd} / \mathrm{MT}$. 


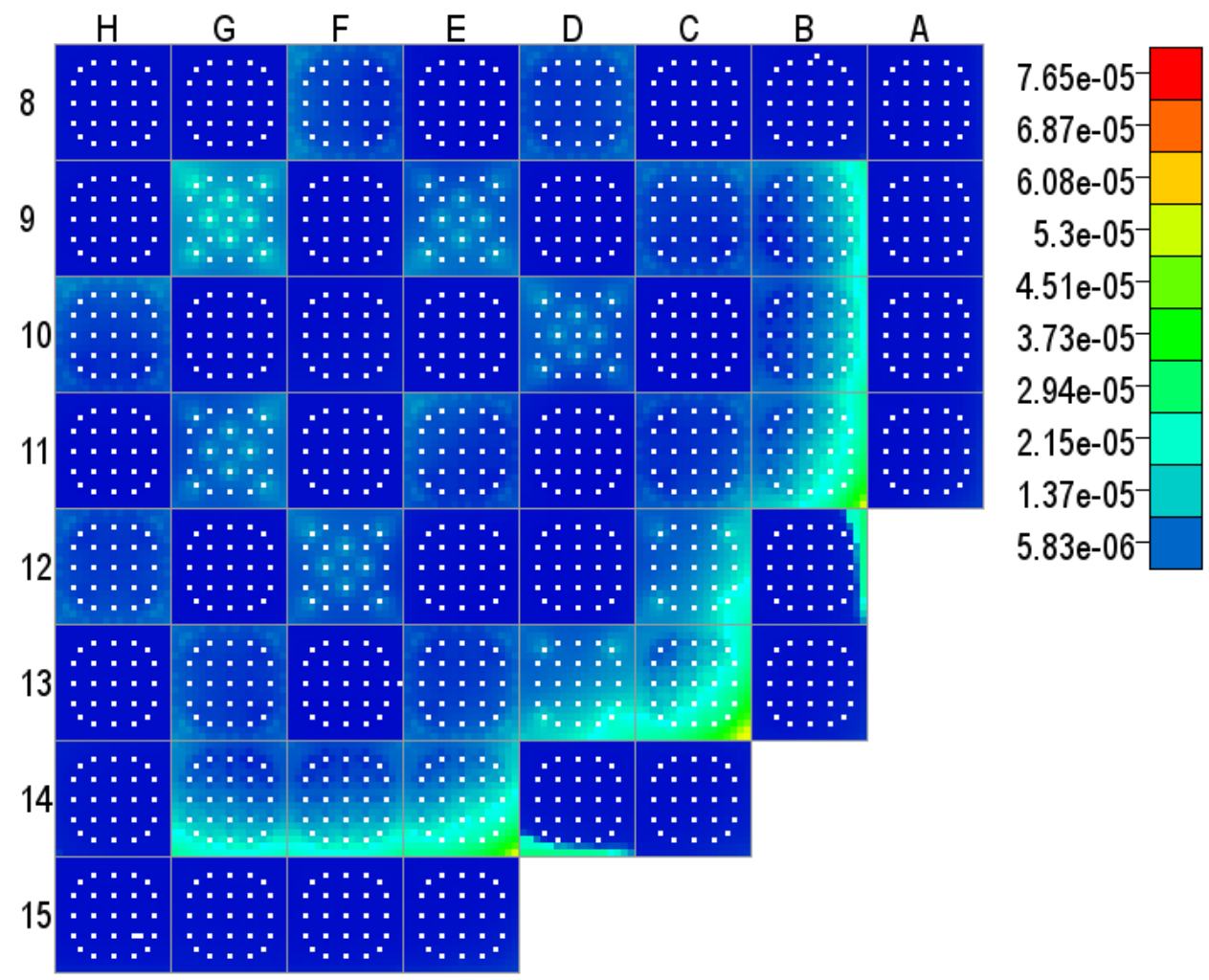

Fig. A.2.33. Cycle 2 minimum gap thickness (m) - 5.53 GWd/MT.

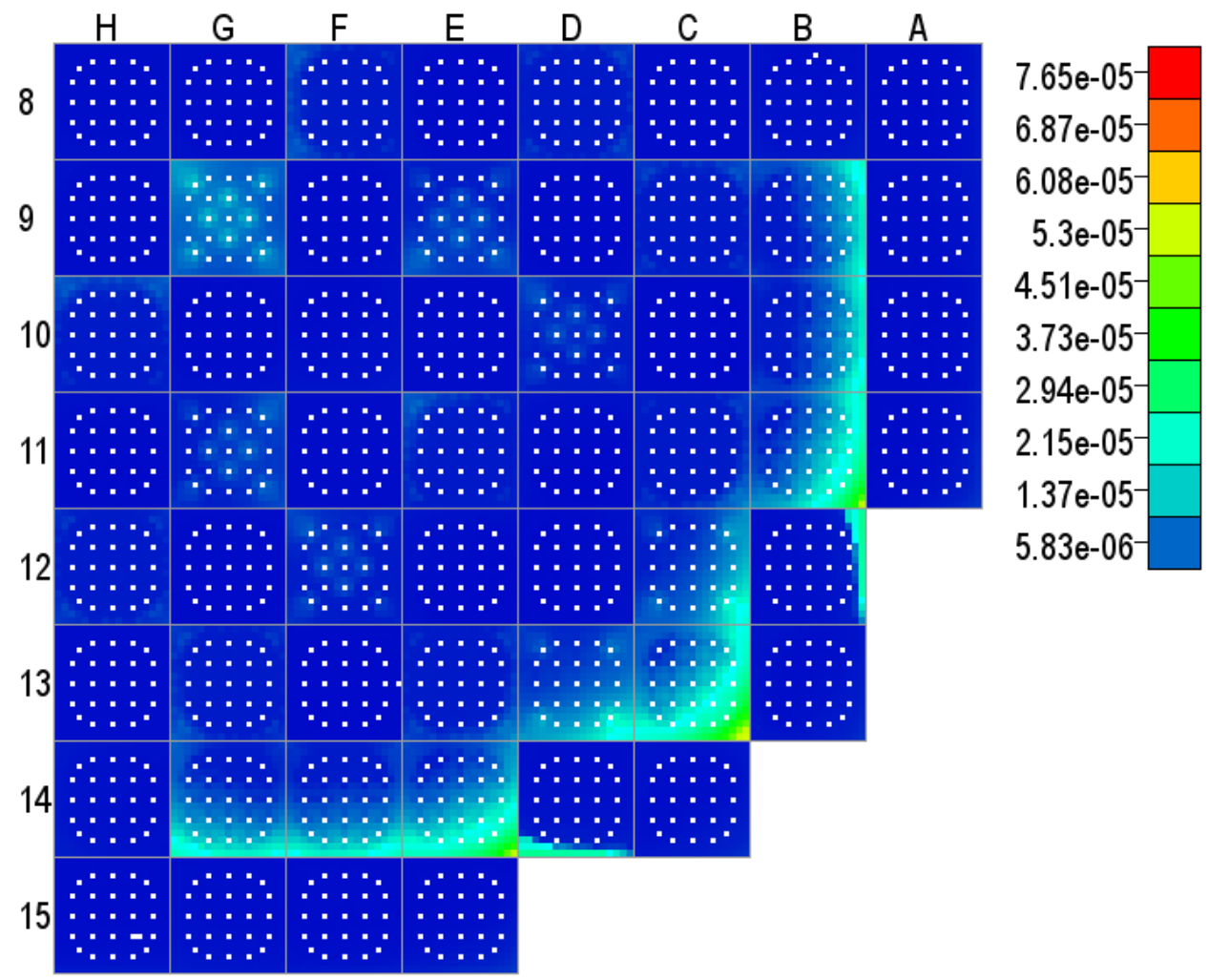

Fig. A.2.34. Cycle 2 minimum gap thickness (m) - 6.53 GWd/MT. 


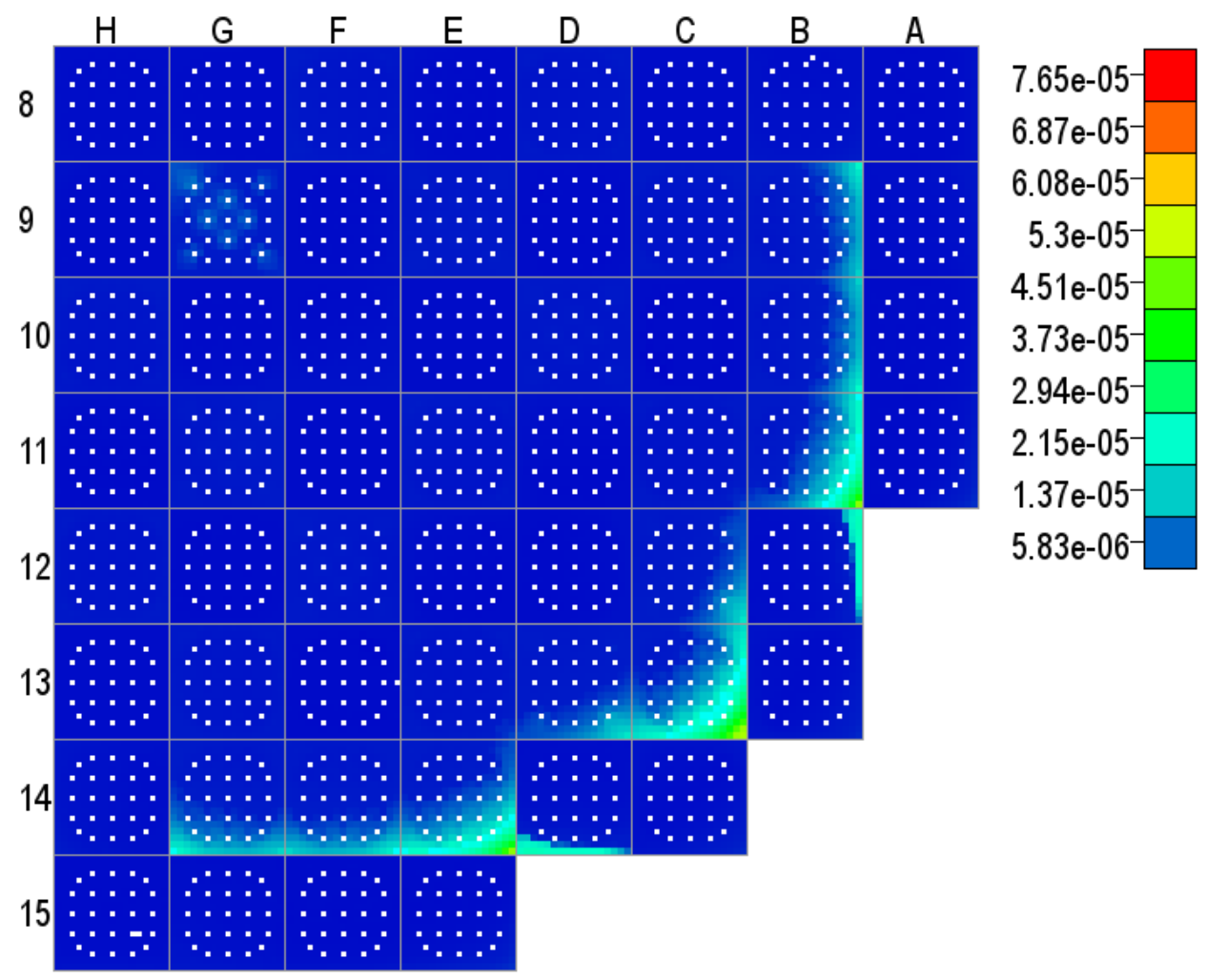

Fig. A.2.35. Cycle 2 minimum gap thickness $(\mathrm{m})-8.22 \mathrm{GWd} / \mathrm{MT}$.

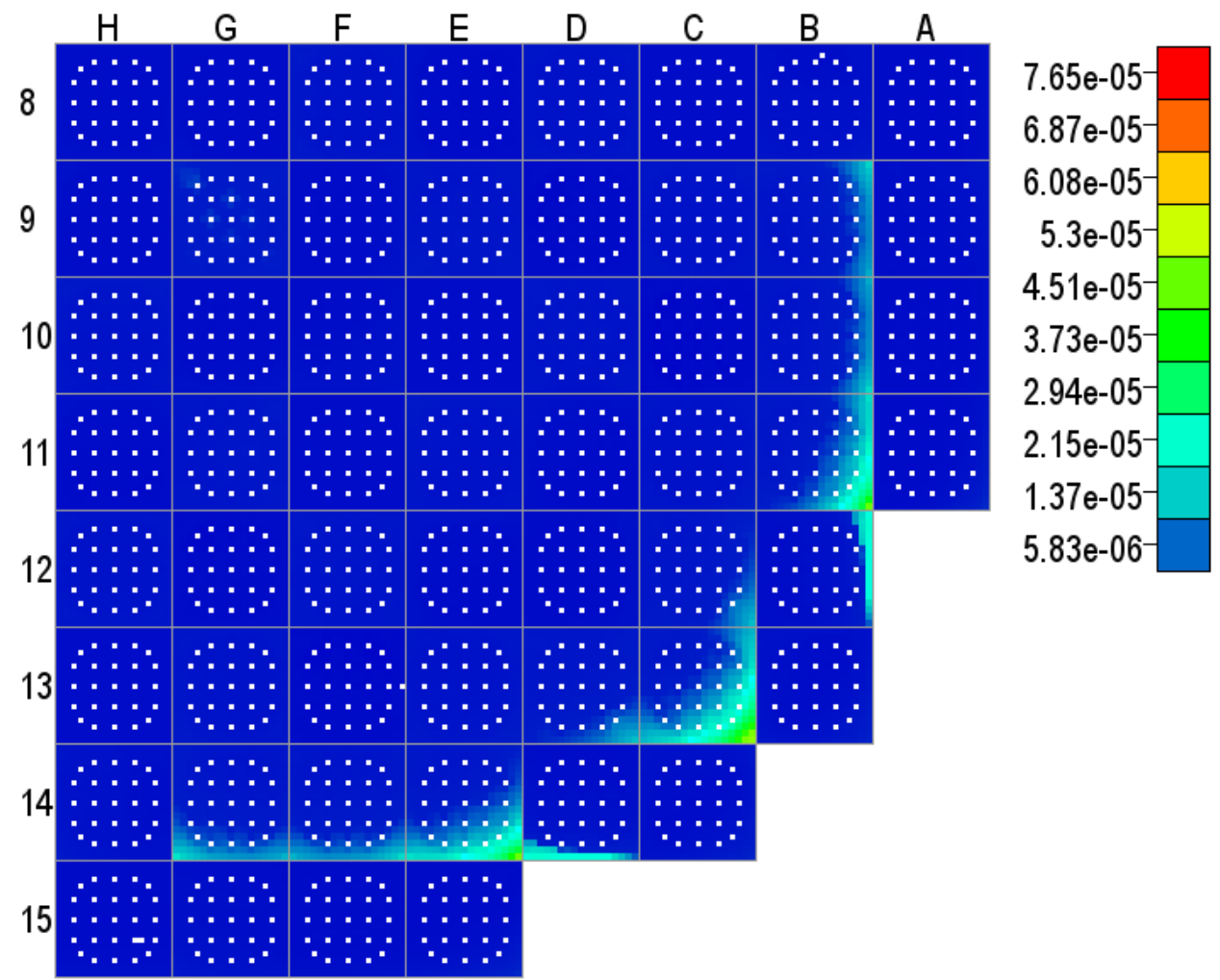

Fig. A.2.36. Cycle 2 minimum gap thickness (m) - 9.29 GWd/MT. 


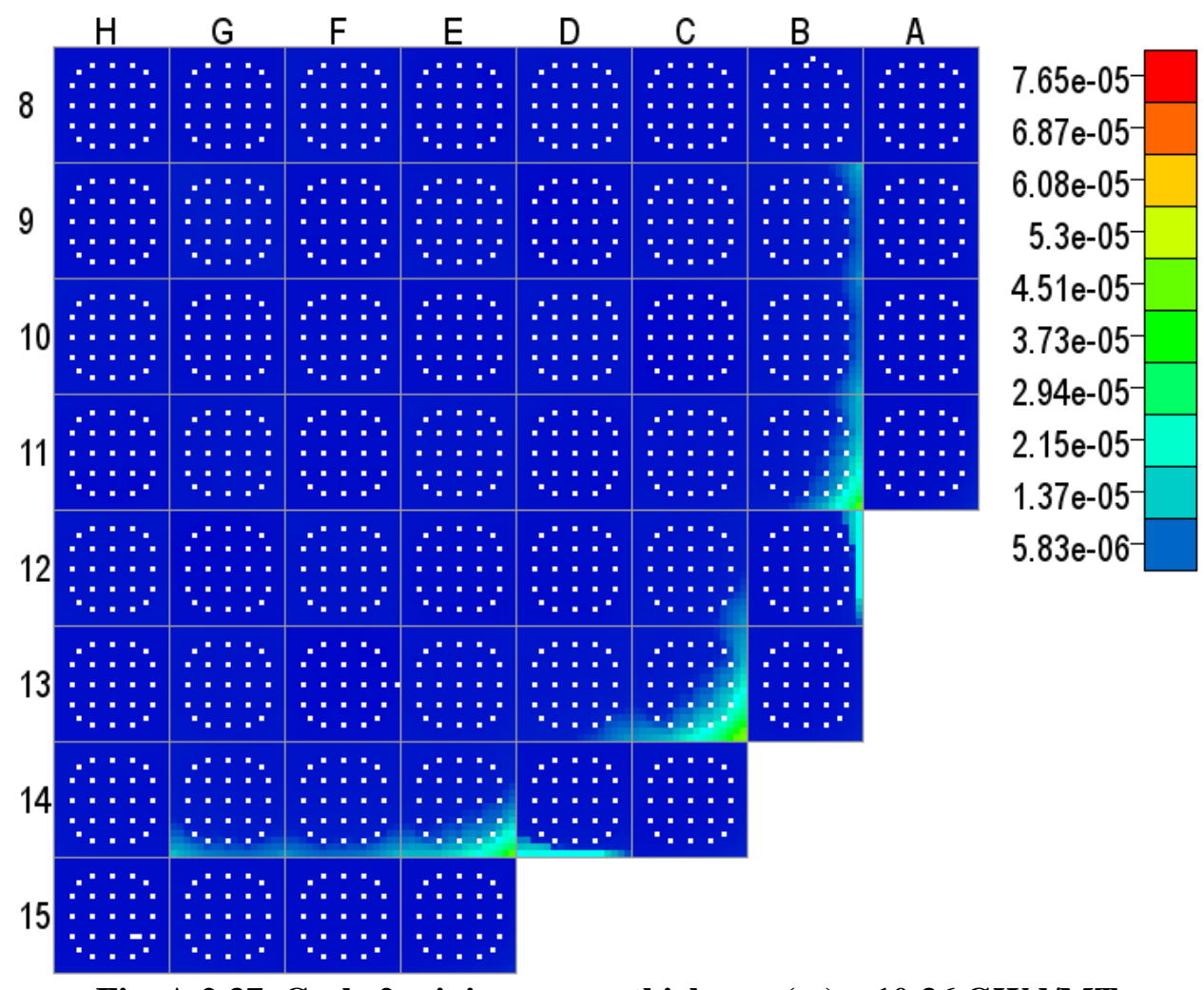

Fig. A.2.37. Cycle 2 minimum gap thickness $(\mathrm{m})-10.36 \mathrm{GWd} / \mathrm{MT}$.

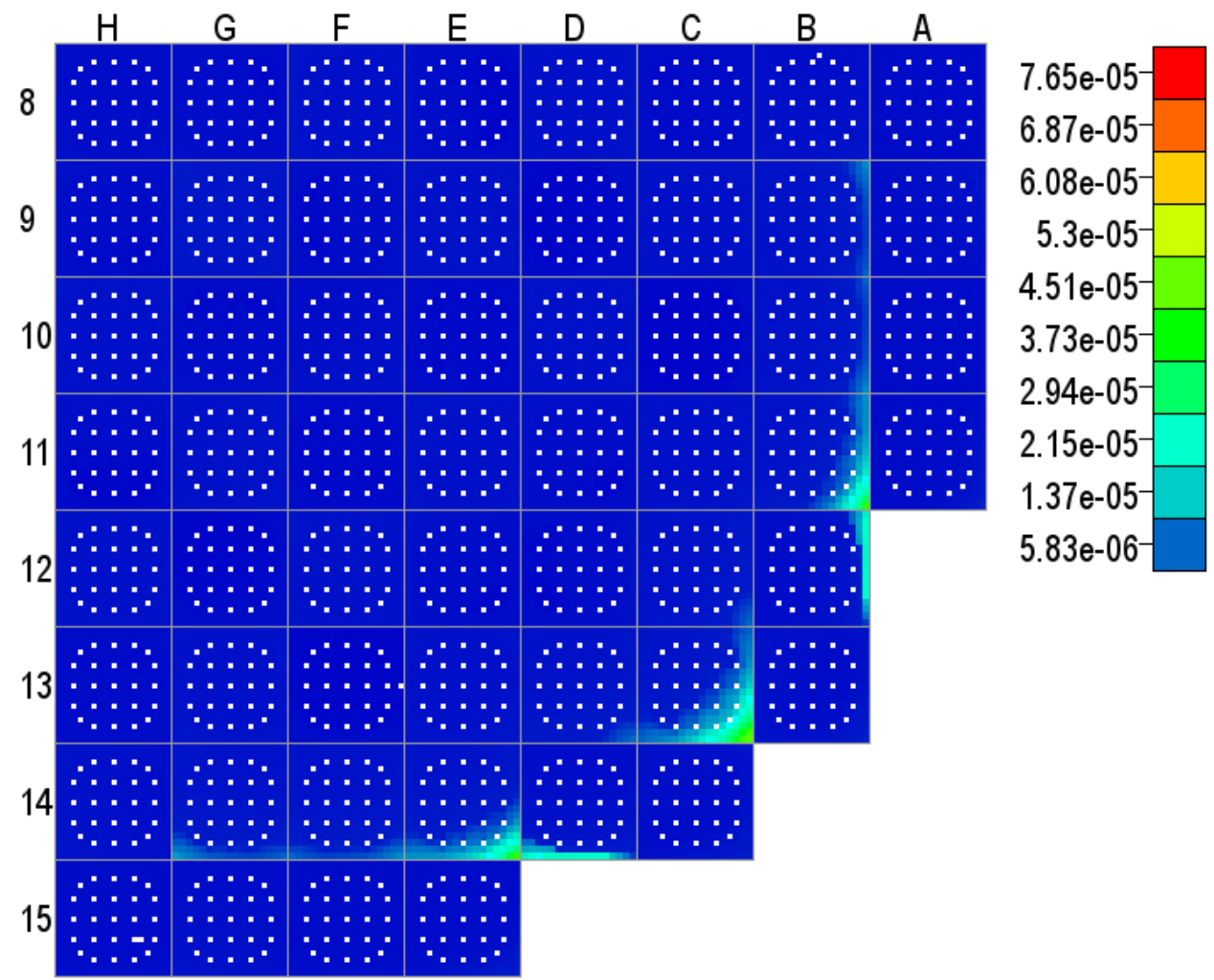

Fig. A.2.38. Cycle 2 minimum gap thickness (m) - 11.43 GWd/MT. 


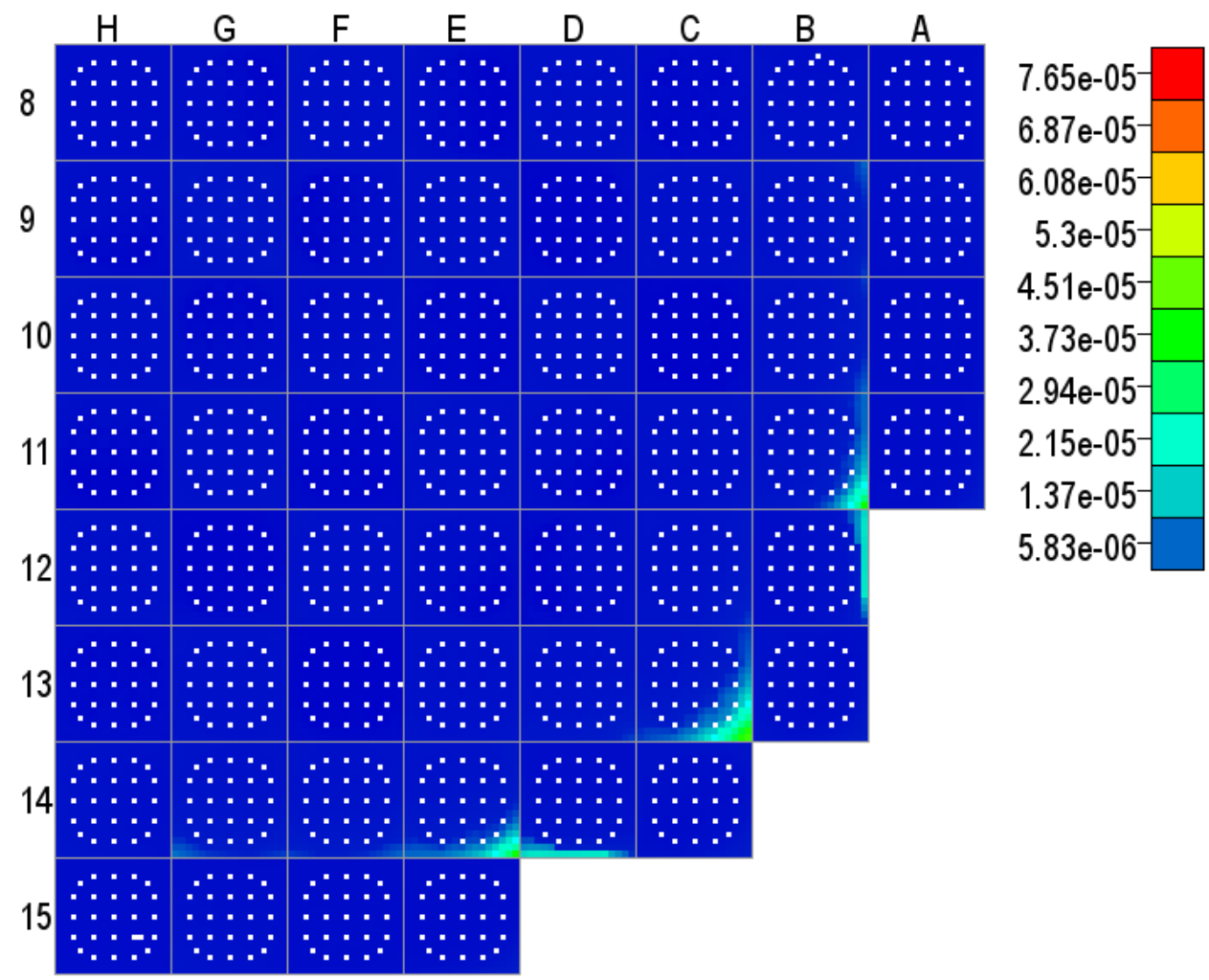

Fig. A.2.39. Cycle 2 minimum gap thickness (m) - 12.54 GWd/MT.

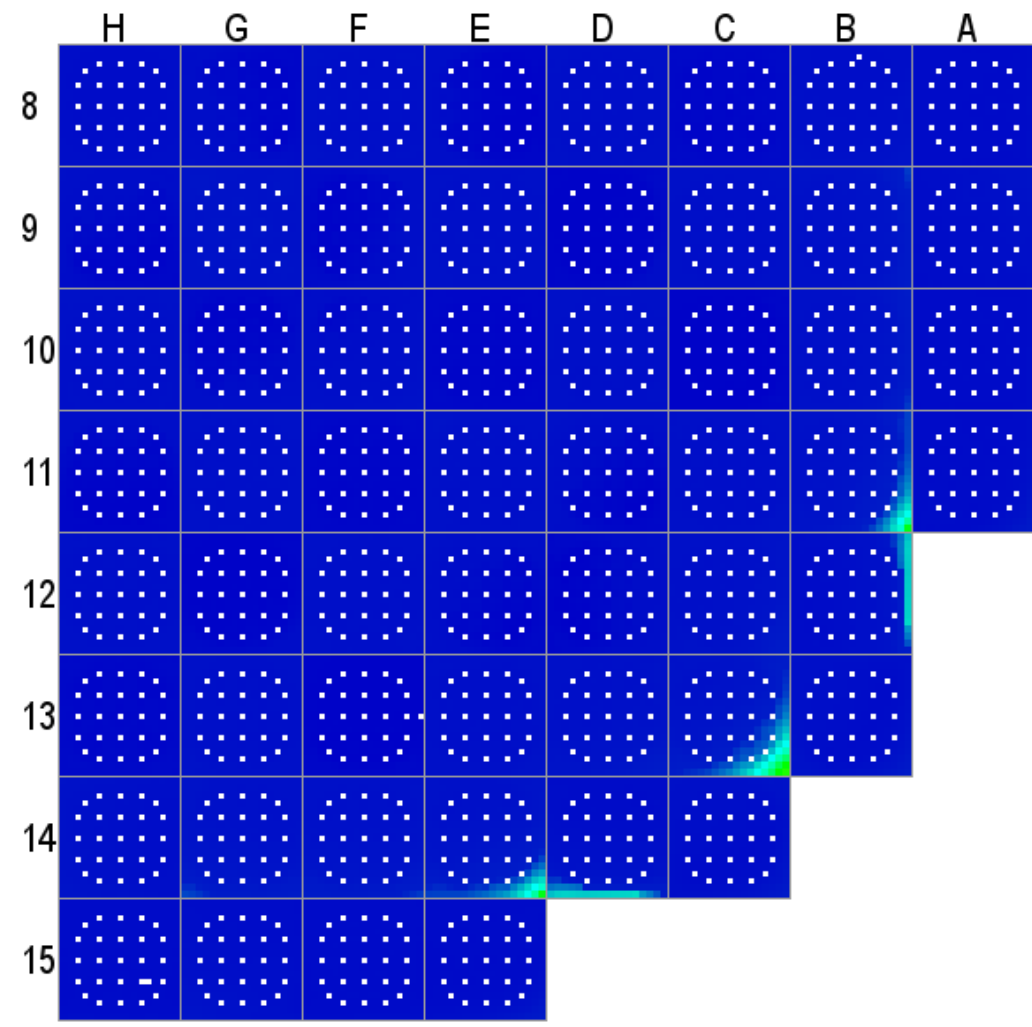

$7.65 \mathrm{e}-05-$
$6.87 \mathrm{e}-05$
$6.08 \mathrm{e}-05$
$5.3 \mathrm{e}-05$
$4.51 \mathrm{e}-05$
$3.73 \mathrm{e}-05$
$2.94 \mathrm{e}-05$
$2.15 \mathrm{e}-05$
$1.37 \mathrm{e}-05$
$5.83 \mathrm{e}-06$

Fig. A.2.40. Cycle 2 minimum gap thickness (m) - 13.54 GWd/MT. 


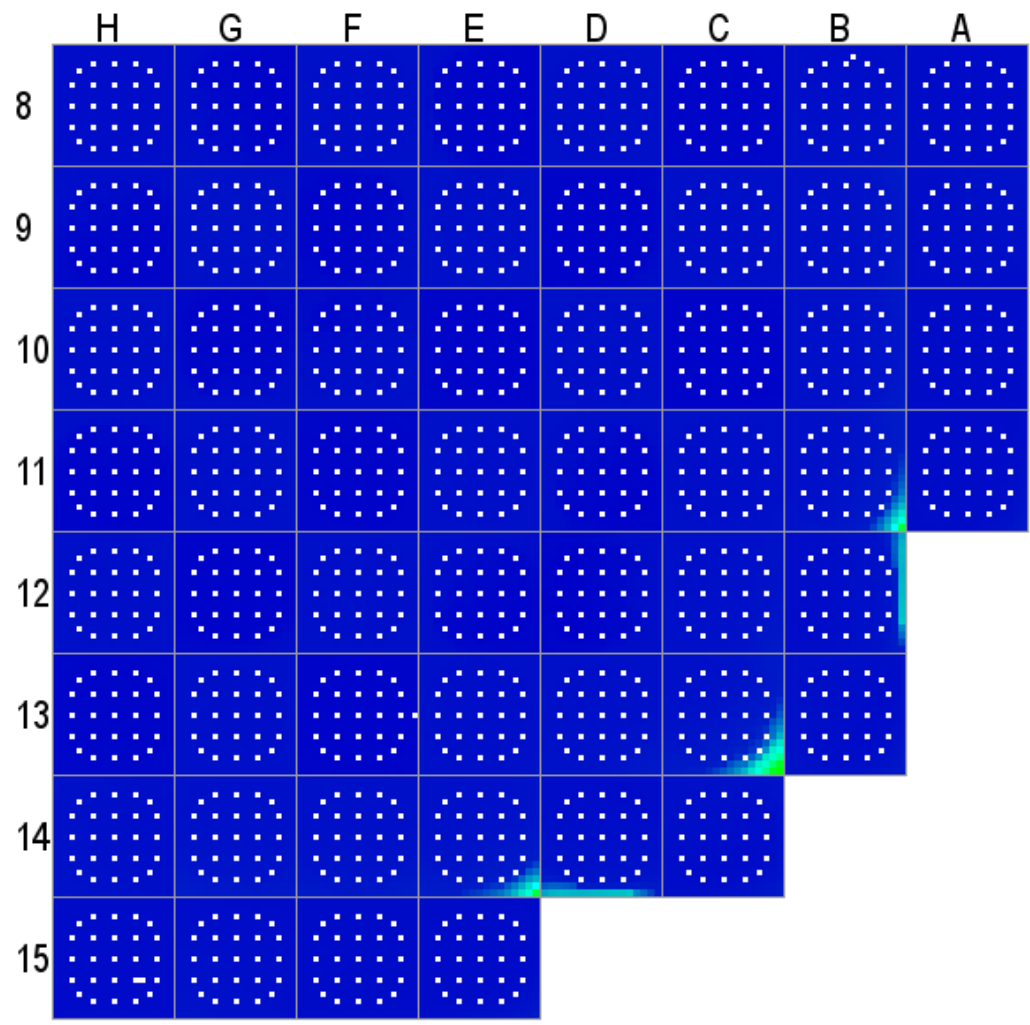

$7.65 \mathrm{e}-05$
$6.87 \mathrm{e}-05$
$6.08 \mathrm{e}-05$
$5.3 \mathrm{e}-05$
$4.51 \mathrm{e}-05$
$3.73 \mathrm{e}-05$
$2.94 \mathrm{e}-05$
$2.15 \mathrm{e}-05$
$1.37 \mathrm{e}-05$
$5.83 \mathrm{e}-06$

Fig. A.2.41. Cycle 2 minimum gap thickness (m) - 14.62 GWd/MT.

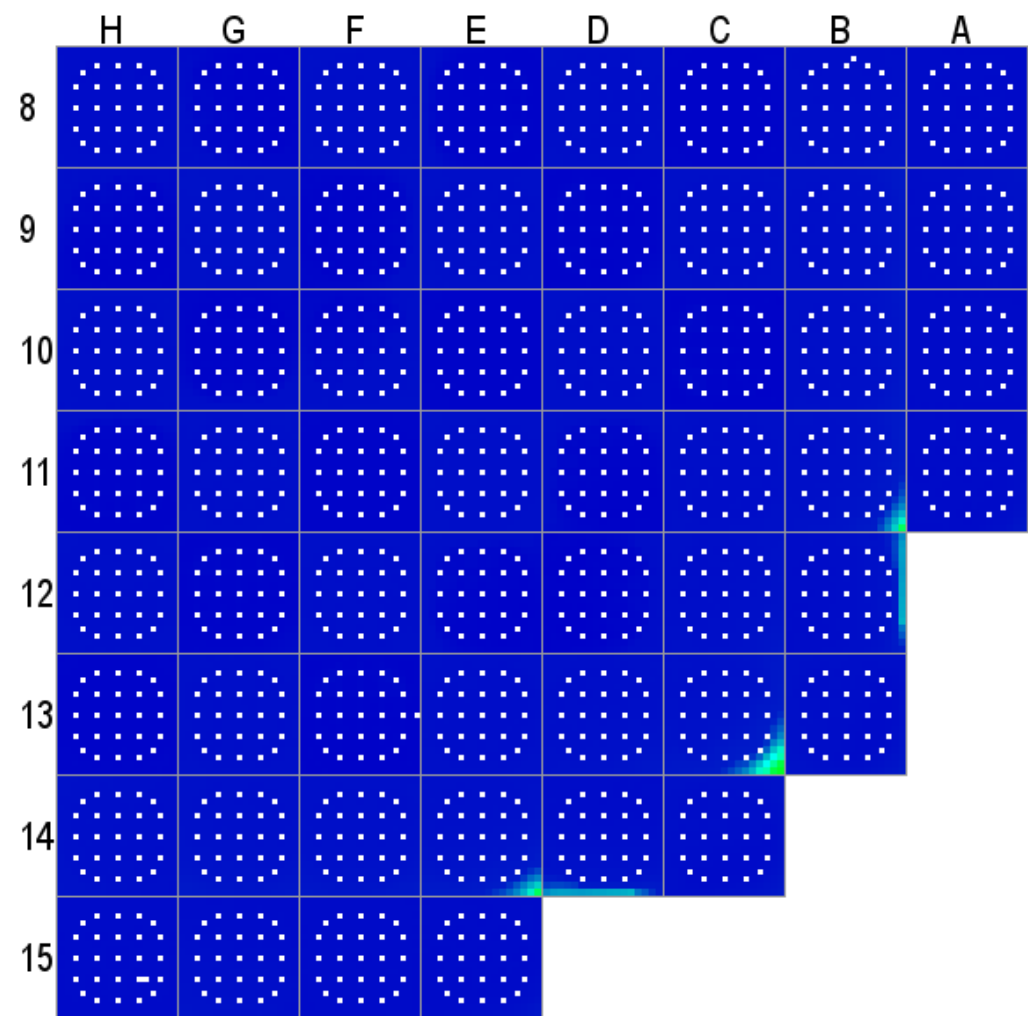

$7.65 \mathrm{e}-05$

$6.87 \mathrm{e}-05$

$6.08 \mathrm{e}-05$

$5.3 \mathrm{e}-05$

$4.51 \mathrm{e}-05$

$3.73 \mathrm{e}-05$

$2.94 \mathrm{e}-05$

$2.15 \mathrm{e}-05$

$1.37 \mathrm{e}-05$

$5.83 \mathrm{e}-06$

Fig. A.2.42. Cycle 2 minimum gap thickness (m) - 15.76 GWd/MT. 


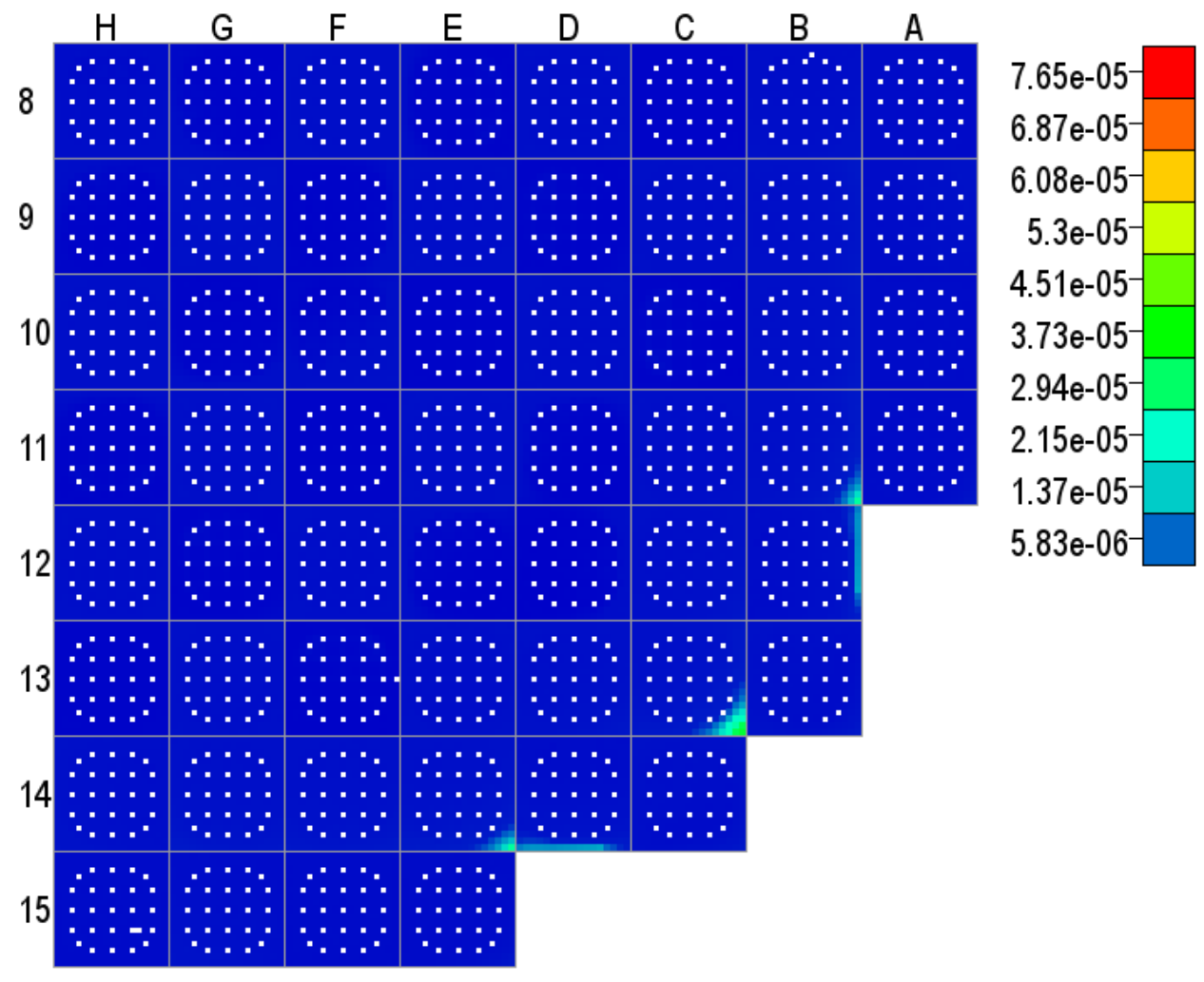

Fig. A.2.43. Cycle 2 minimum gap thickness $(m)-16.32$ GWd/MT.

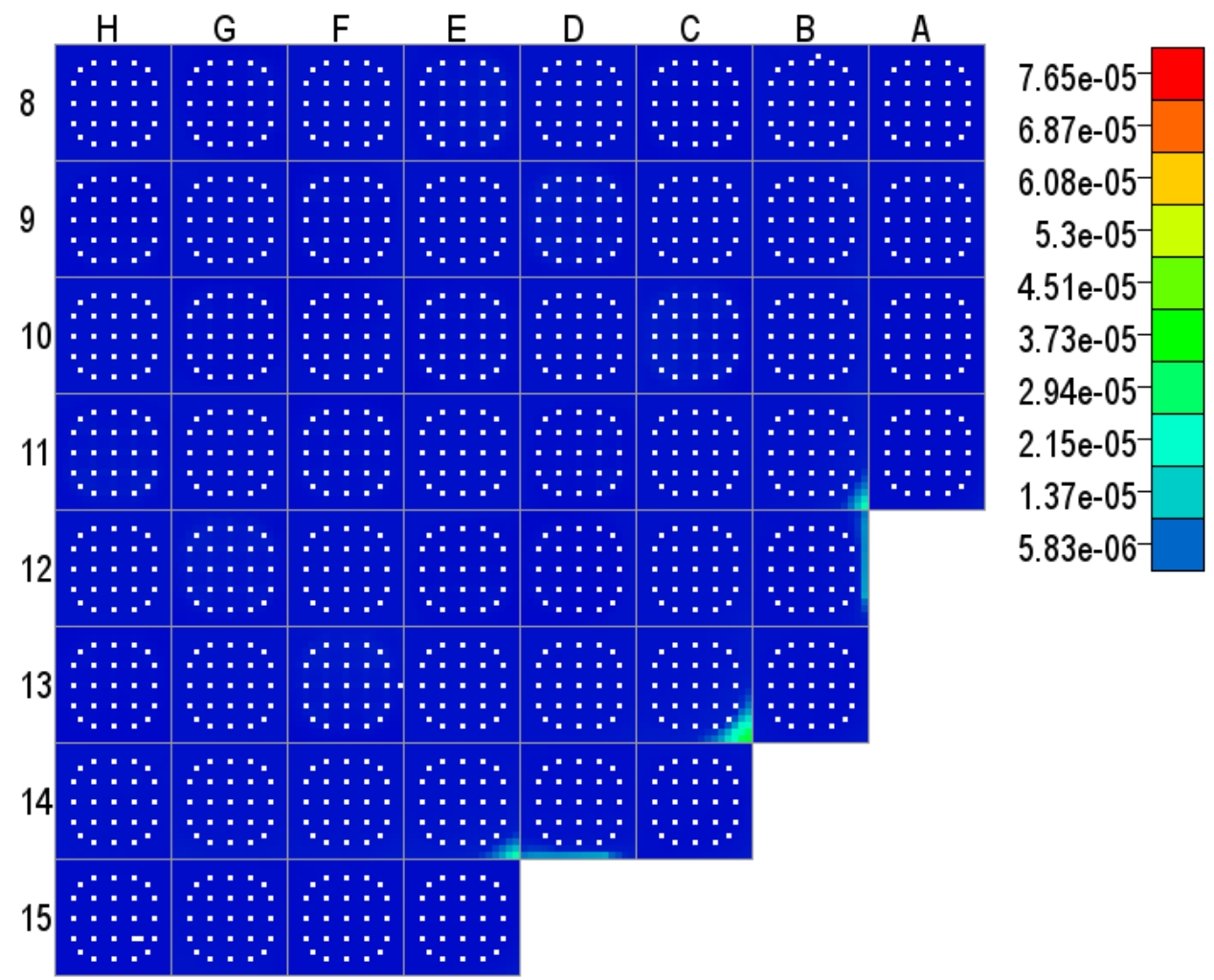

Fig. A.2.44. Cycle 2 minimum gap thickness (m) - 16.90 GWd/MT. 


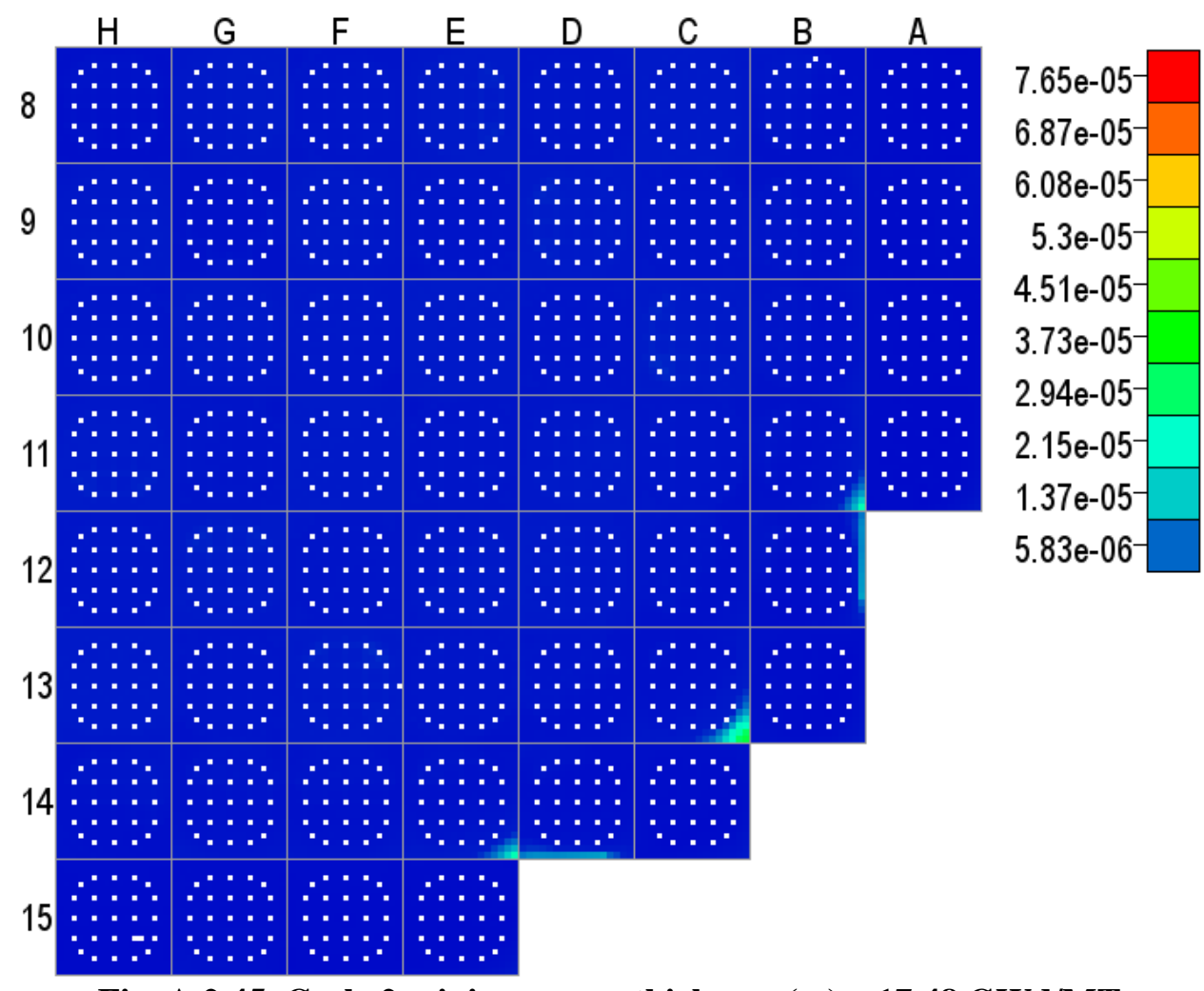

Fig. A.2.45. Cycle 2 minimum gap thickness (m) - 17.48 GWd/MT.

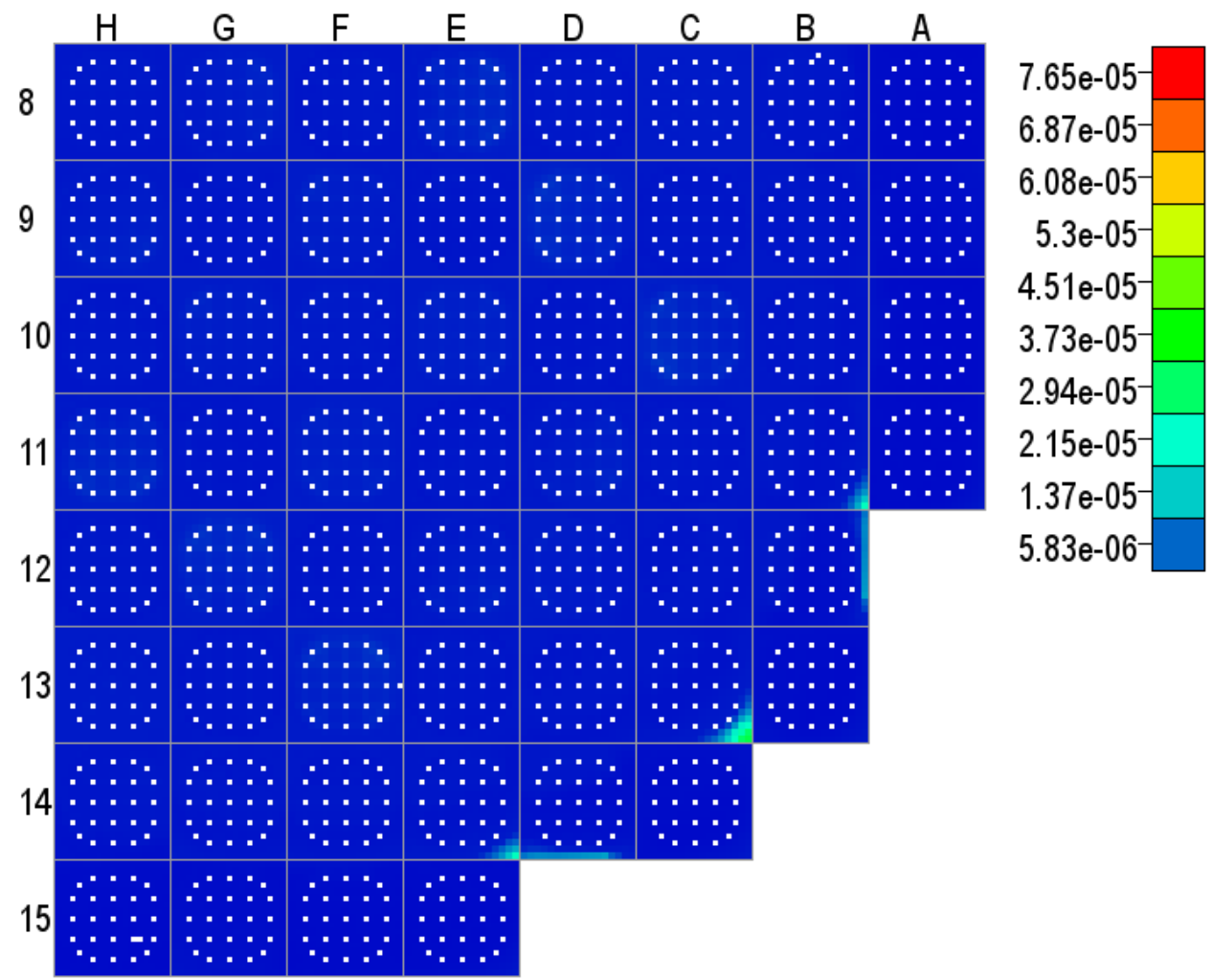

Fig. A.2.46. Cycle 2 minimum gap thickness $(\mathrm{m})-18.06 \mathrm{GWd} / \mathrm{MT}$. 


\section{A.3. Cycle 3}

\section{A.3.1. Maximum Centerline Fuel Temperature}

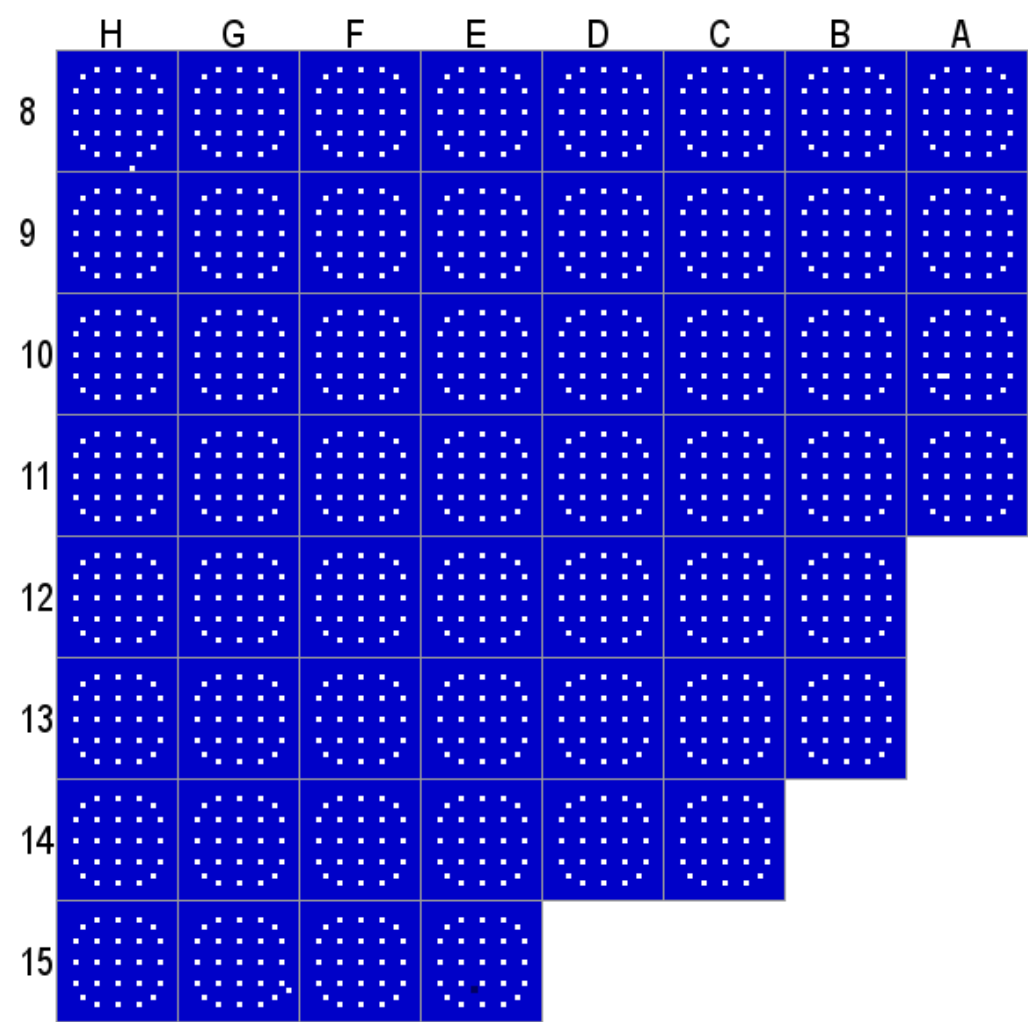

$1507.02-$
1412.8
1318.58
1224.36
1130.15
1035.93
942
-147
$753-$
$659-$

Fig. A.3.1. Cycle 3 maximum centerline fuel temperature $(\mathrm{K})-0.00 \mathrm{GWd} / \mathrm{MT}$.
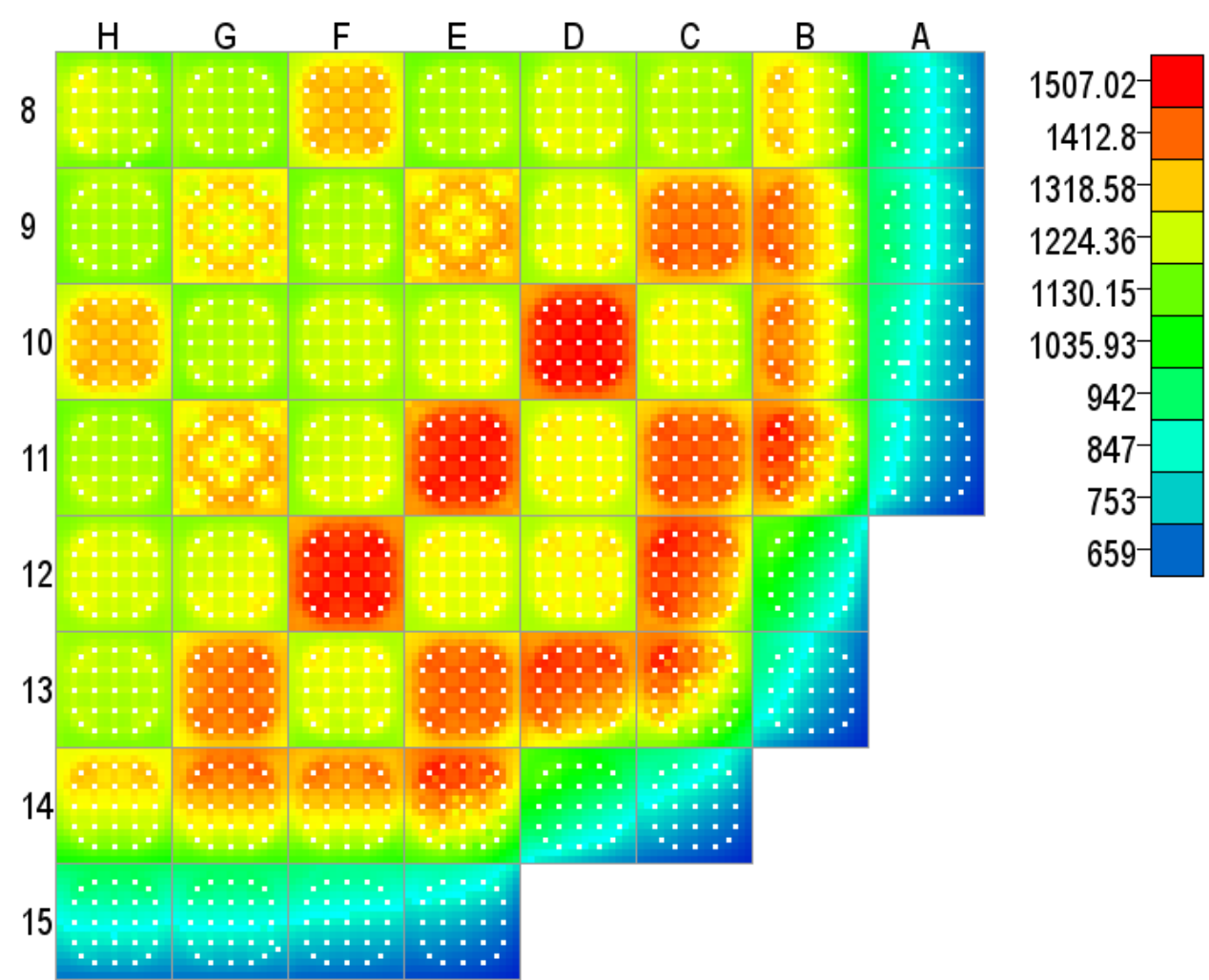

Fig. A.3.2. Cycle 3 maximum centerline fuel temperature $(\mathrm{K})-0.20 \mathrm{GWd} / \mathrm{MT}$. 

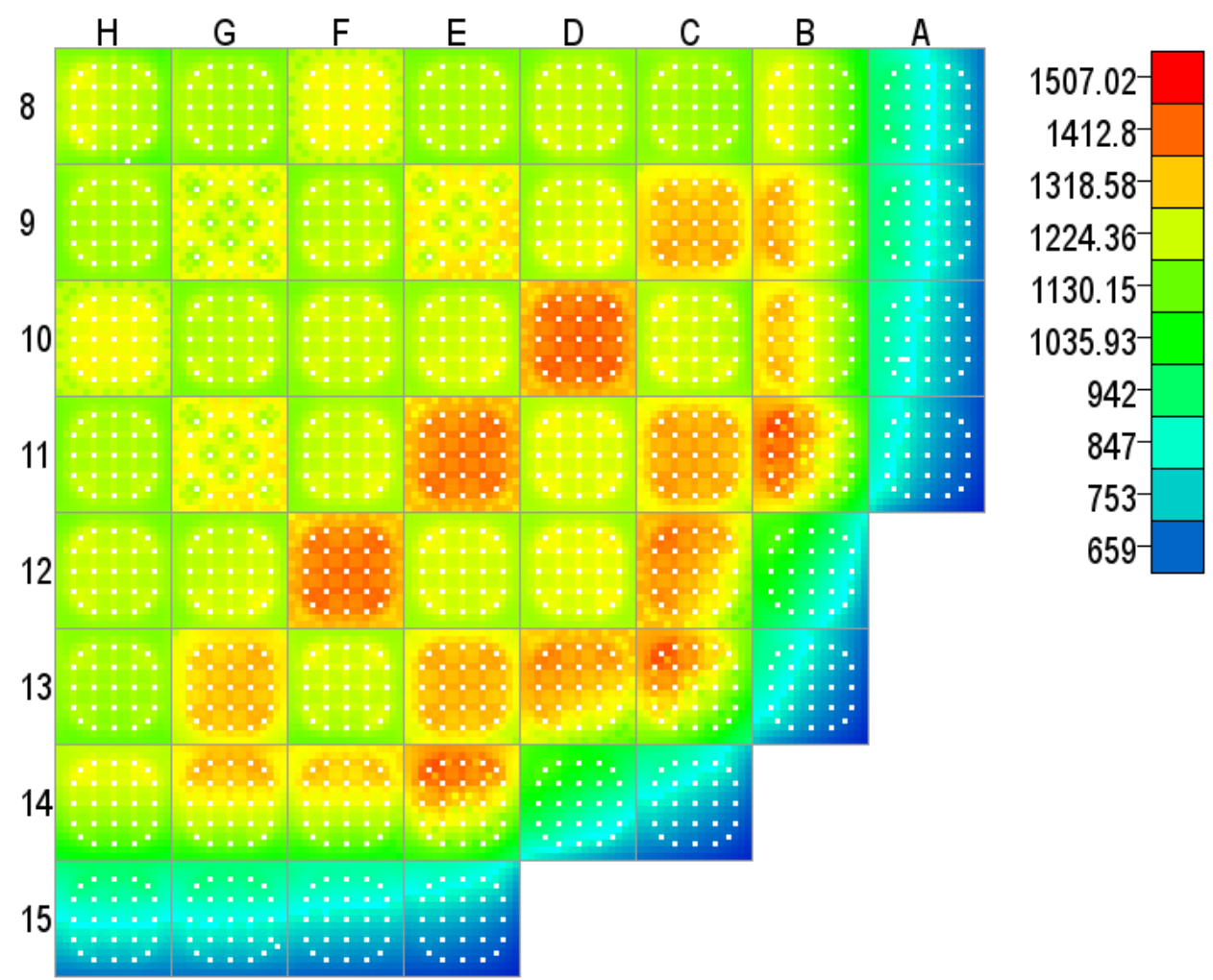

Fig. A.3.3. Cycle 3 maximum centerline fuel temperature $(\mathrm{K})-0.69 \mathrm{GWd} / \mathrm{MT}$.

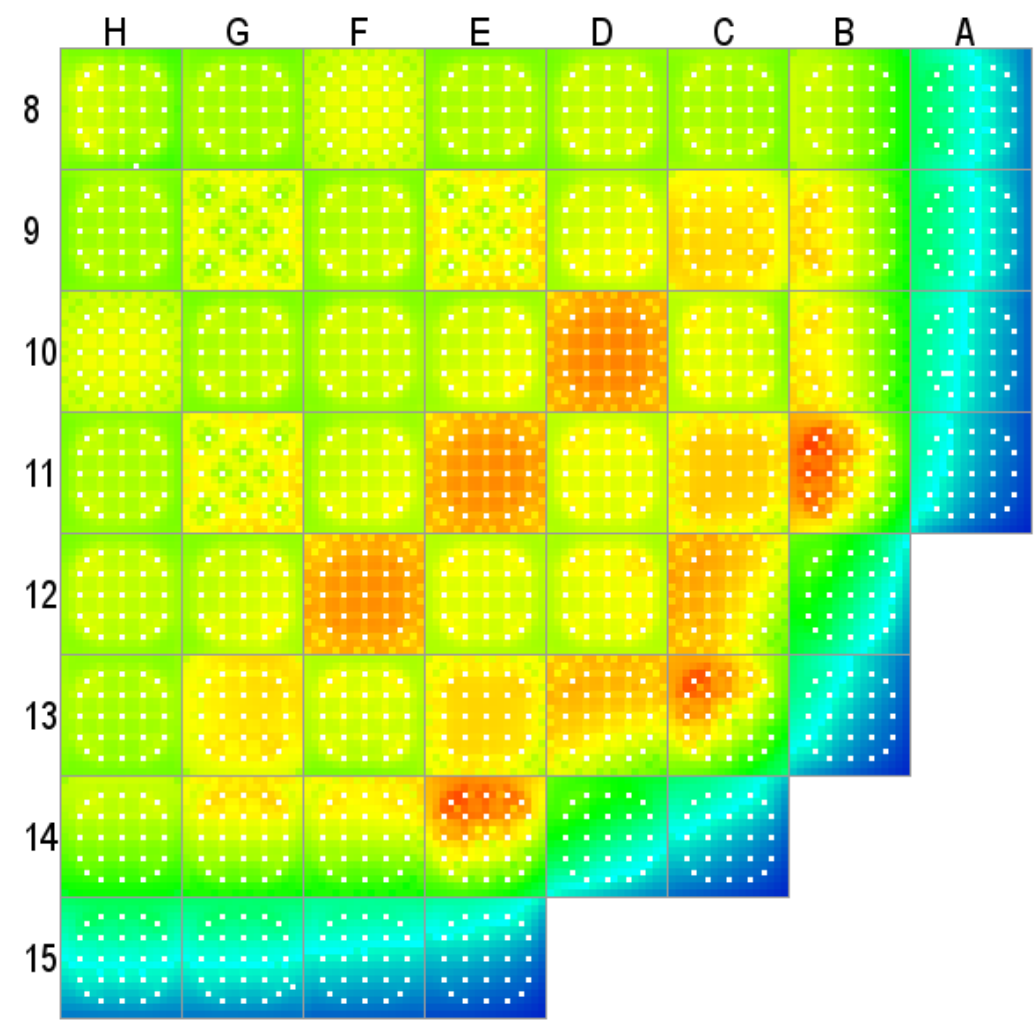

$1507.02-$
1412.8
1318.58
1224.36
1130.15
1035.93
$942-$
847
$753-$
-
$659-$

Fig. A.3.4. Cycle 3 maximum centerline fuel temperature $(\mathrm{K})-1.17 \mathrm{GWd} / \mathrm{MT}$. 


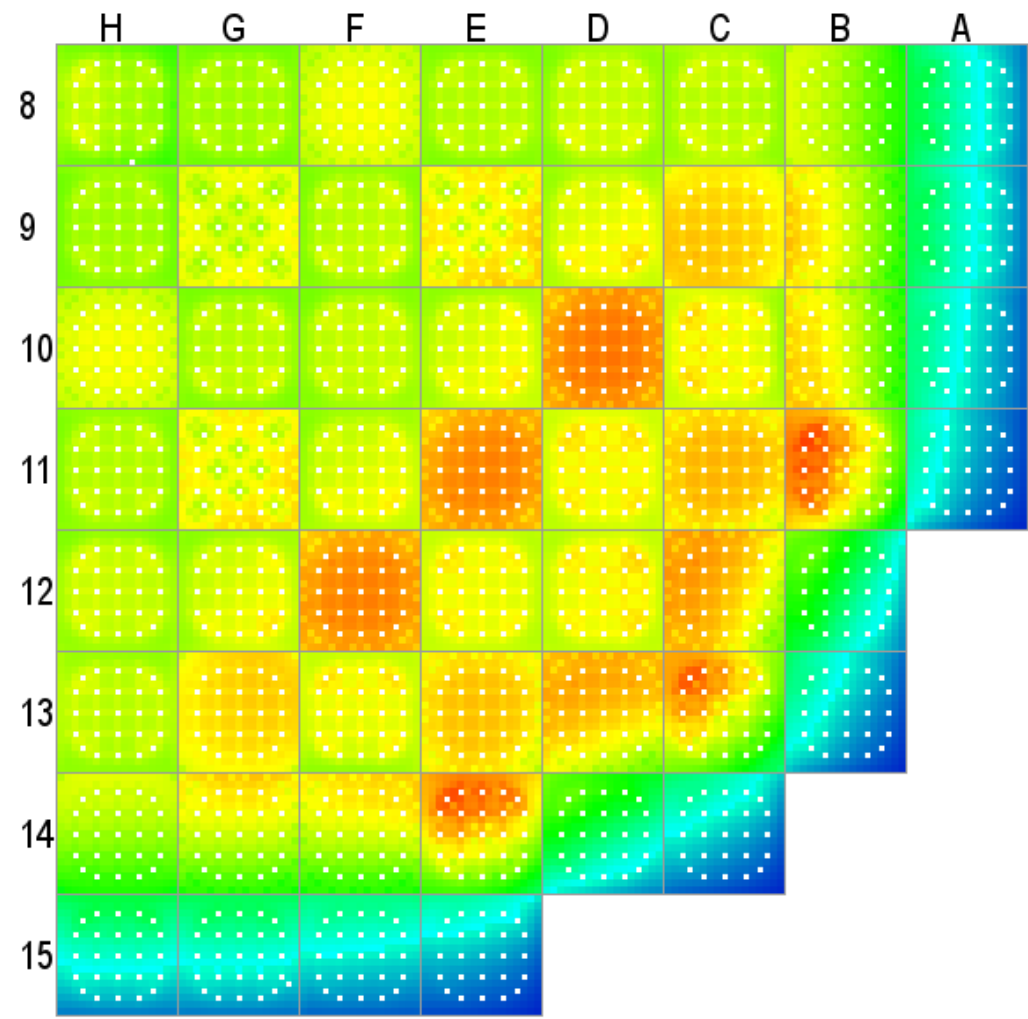

1507.02

1412.8

1318.58

1224.36

1130.15

1035.93

942

847

753

$659-$

Fig. A.3.5. Cycle 3 maximum centerline fuel temperature $(\mathrm{K})-1.70 \mathrm{GWd} / \mathrm{MT}$.

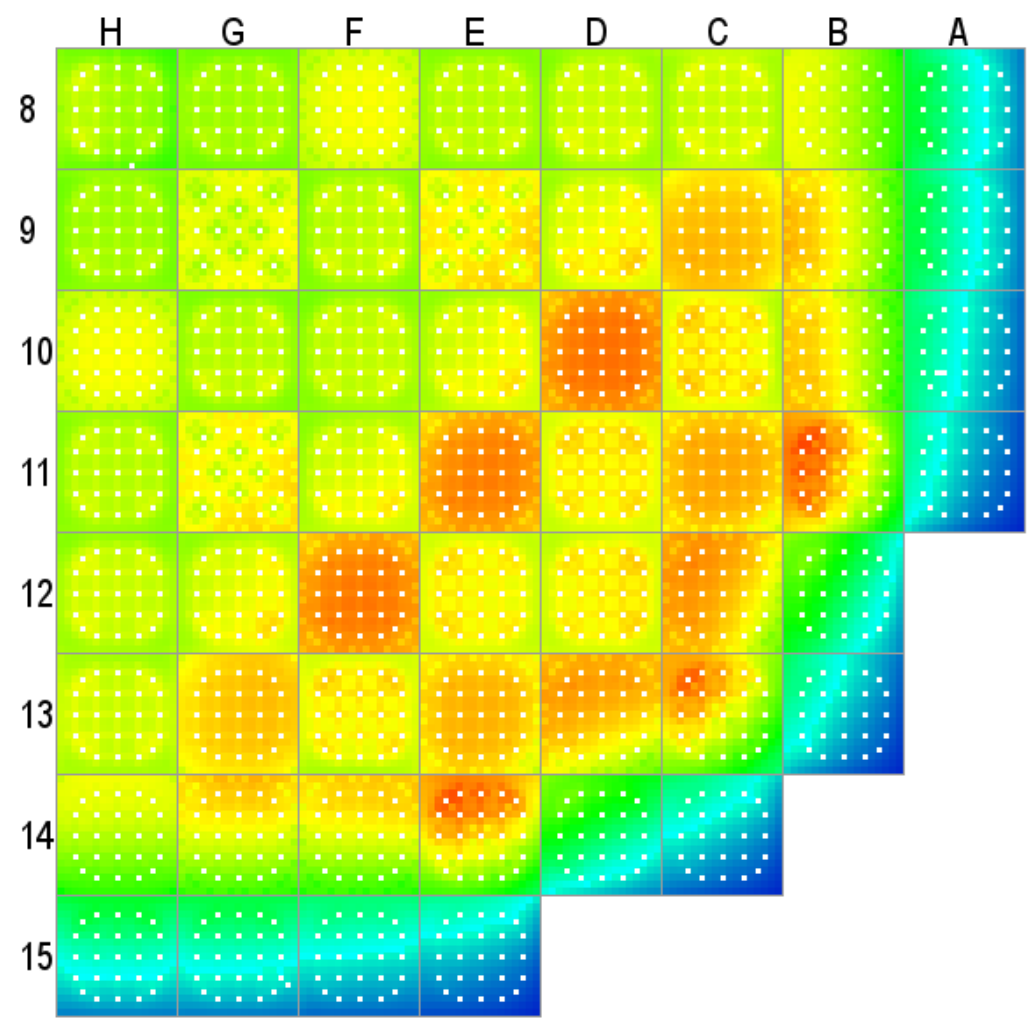

1507.02

1412.8

1318.58

1224.36

1130.15

1035.93

942

$847-$

$753-$

659

Fig. A.3.6. Cycle 3 maximum centerline fuel temperature $(\mathrm{K})-2.24$ GWd/MT. 

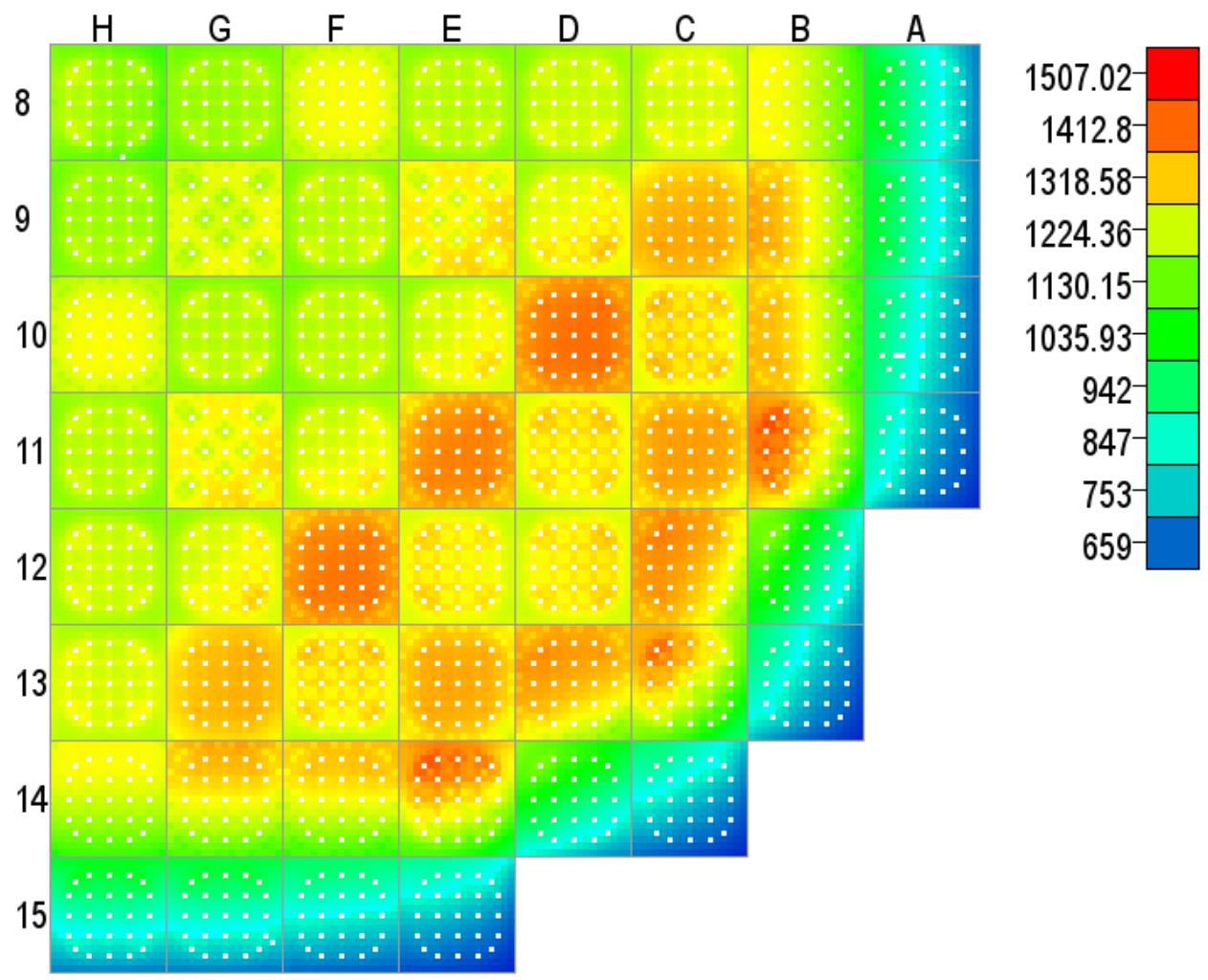

Fig. A.3.7. Cycle 3 maximum centerline fuel temperature $(\mathrm{K})-2.77 \mathrm{GWd} / \mathrm{MT}$.

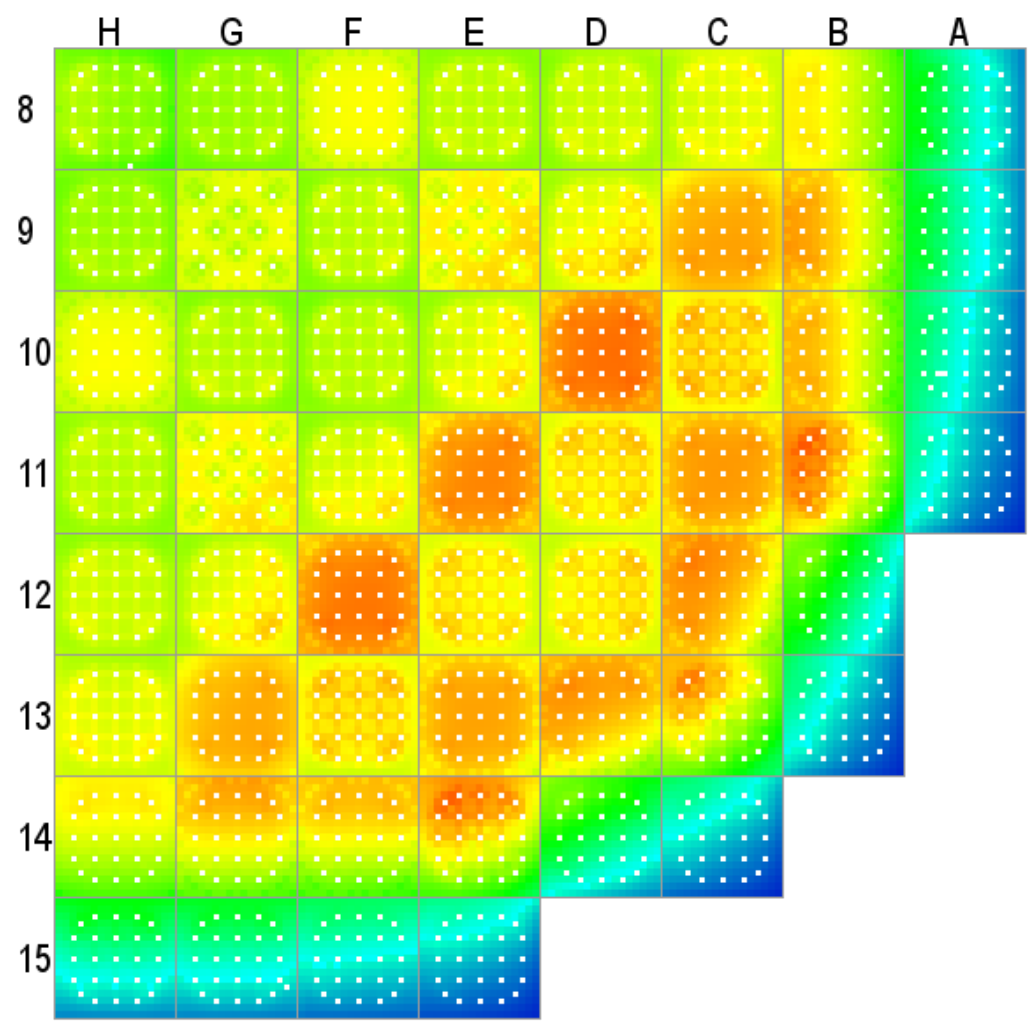

1507.02

1412.8

1318.58

1224.36

1130.15

1035.93

$942-$

847

753

$659-$

Fig. A.3.8. Cycle 3 maximum centerline fuel temperature $(\mathrm{K})-3.30 \mathrm{GWd} / \mathrm{MT}$. 

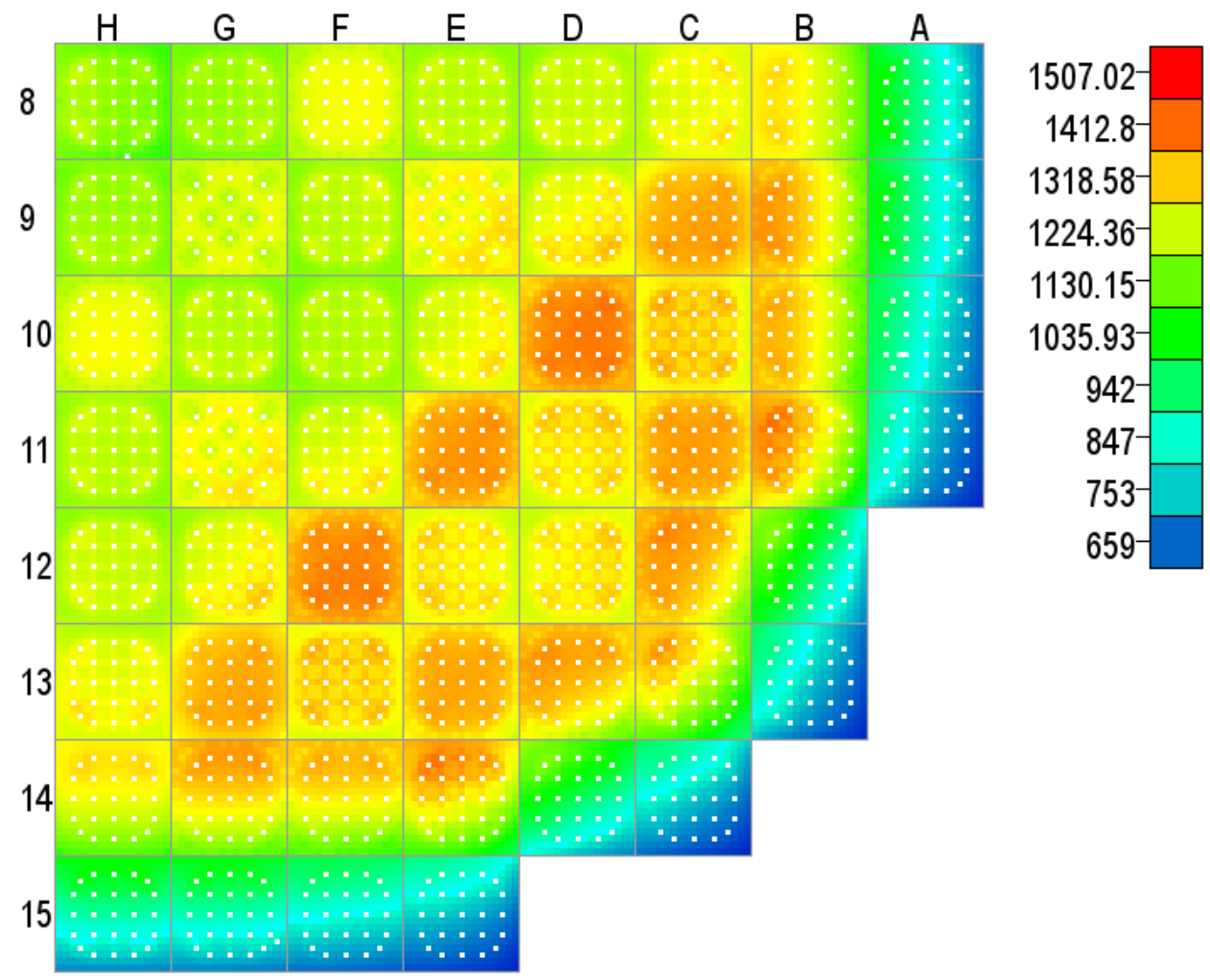

Fig. A.3.9. Cycle 3 maximum centerline fuel temperature $(\mathrm{K})$ - 3.96 GWd/MT.

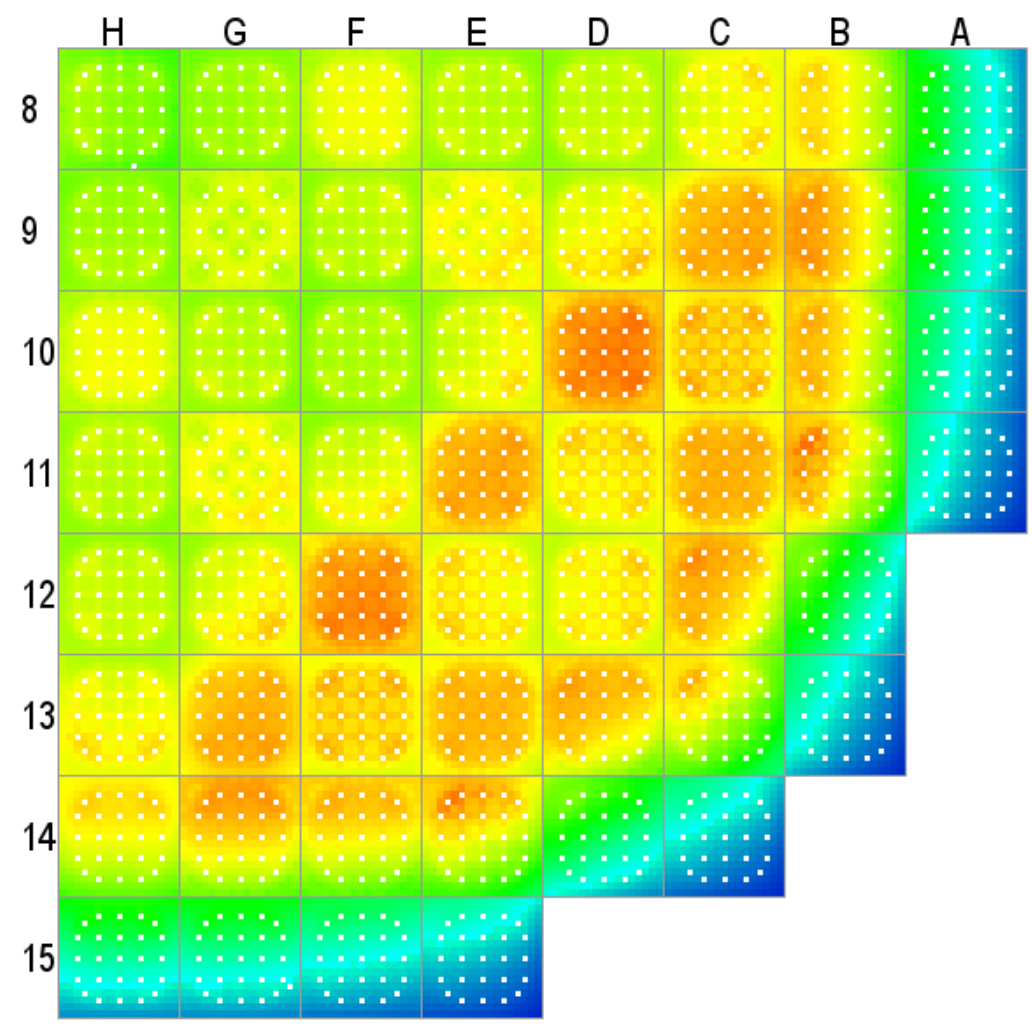

1507.02

1412.8

1318.58

1224.36

1130.15

$1035.93-$

942

$847-$

$753-$

$659-$

Fig. A.3.10. Cycle 3 maximum centerline fuel temperature $(\mathrm{K})$ - 4.91 GWd/MT. 

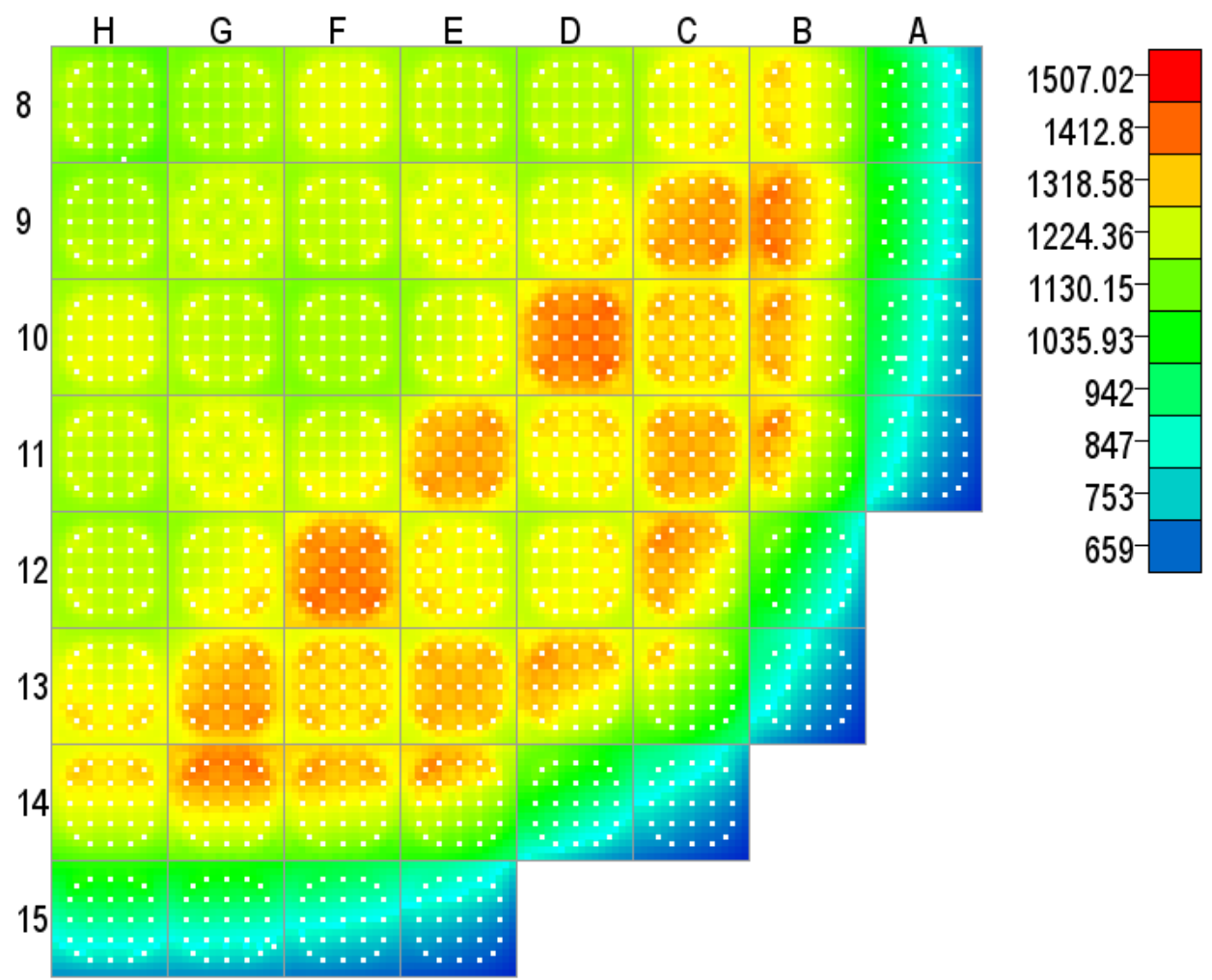

Fig. A.3.11. Cycle 3 maximum centerline fuel temperature $(\mathrm{K})-6.44 \mathrm{GWd} / \mathrm{MT}$.

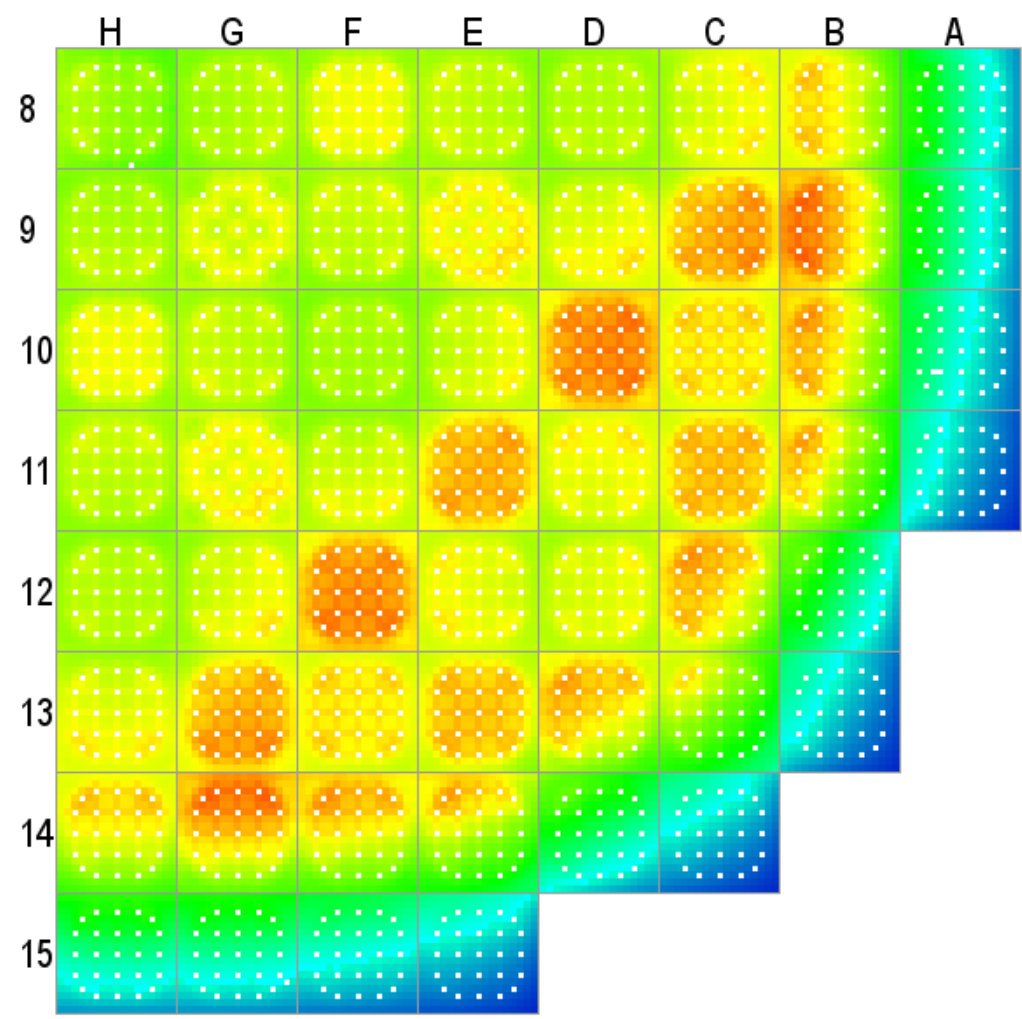

1507.02
$1412.8-$
1318.58
1224.36
1130.15
1035.93
942
847
$753-$
$659-$

Fig. A.3.12. Cycle 3 maximum centerline fuel temperature (K) - 8.13 GWd/MT. 

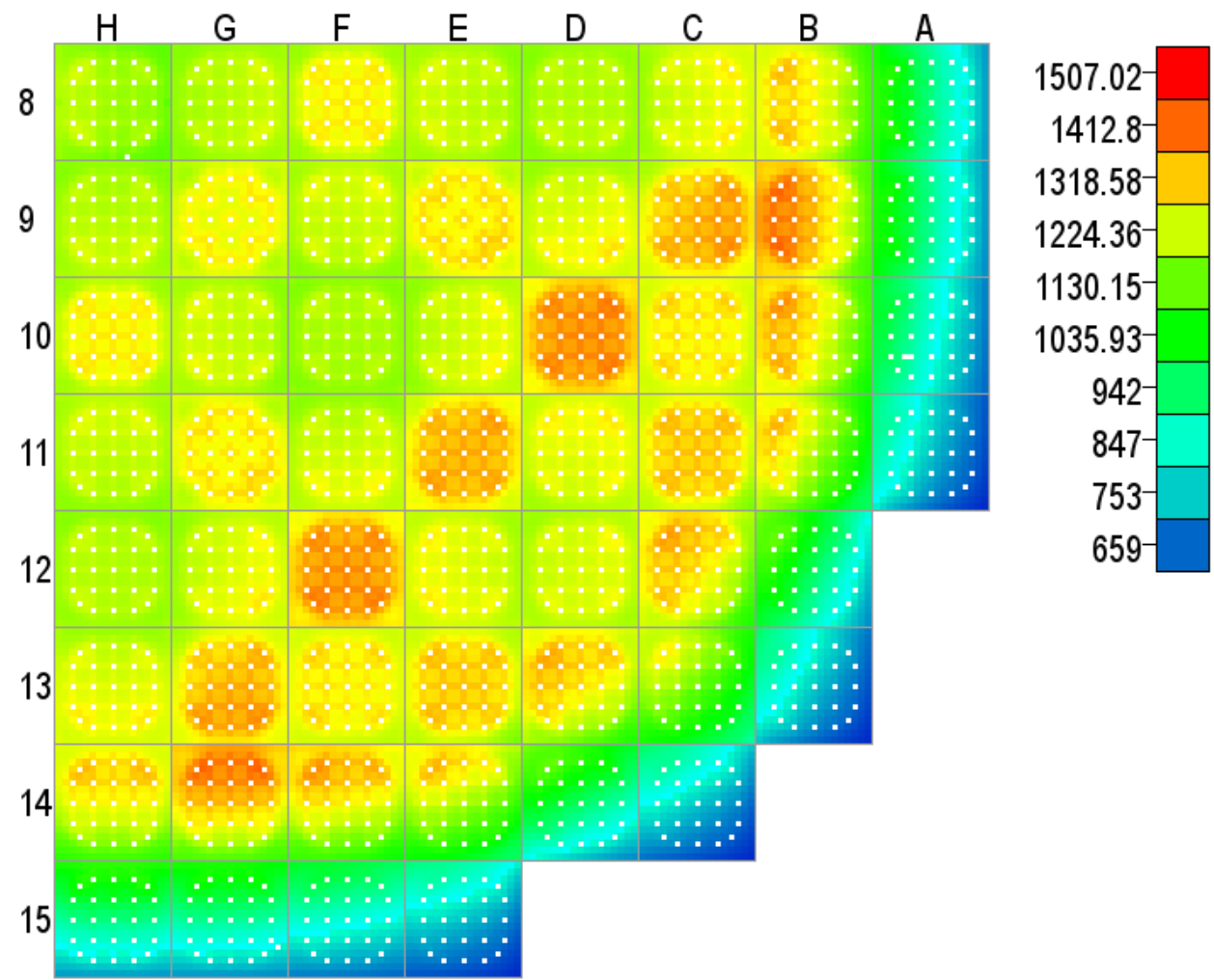

Fig. A.3.13. Cycle 3 maximum centerline fuel temperature (K) - 9.72 GWd/MT.

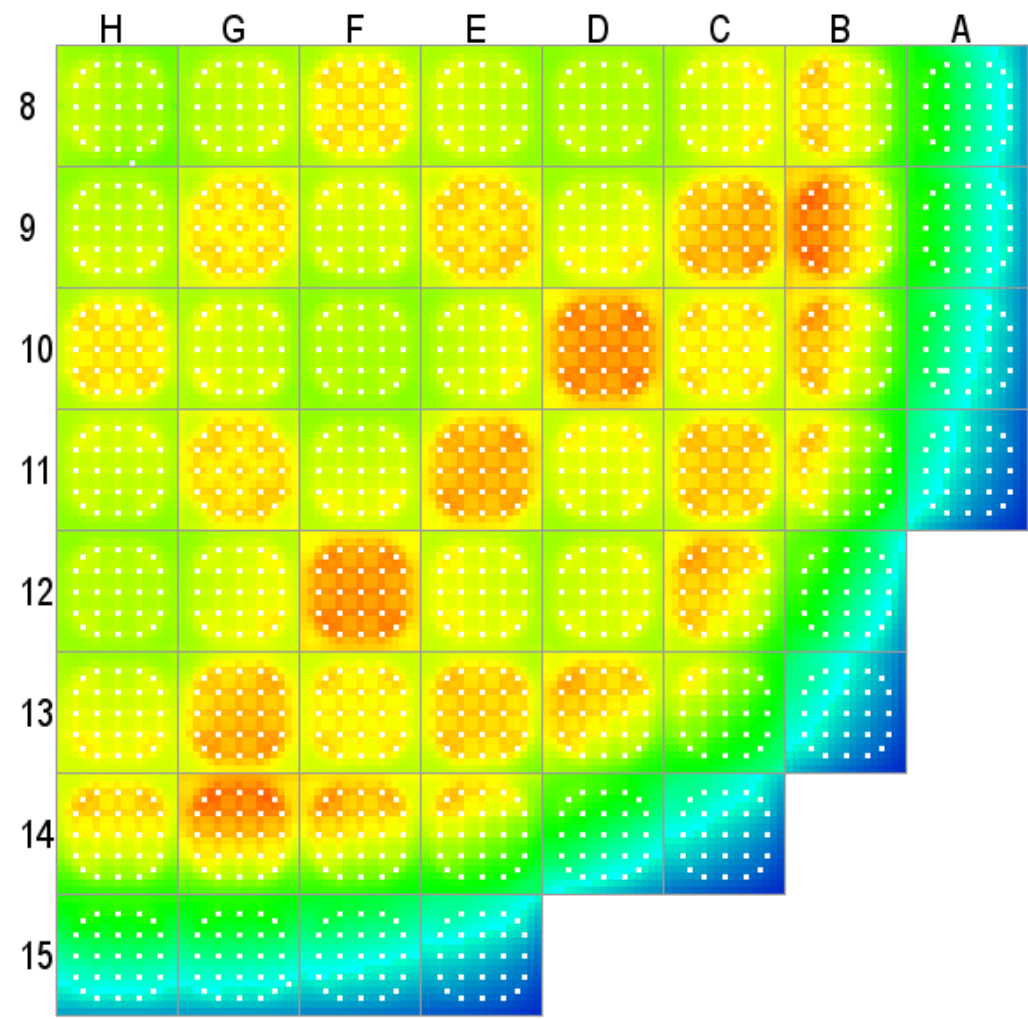

1507.02

1412.8

1318.58

1224.36

1130.15

1035.93

942

$847-$

753

659

Fig. A.3.14. Cycle 3 maximum centerline fuel temperature $(\mathrm{K})-11.35$ GWd/MT. 


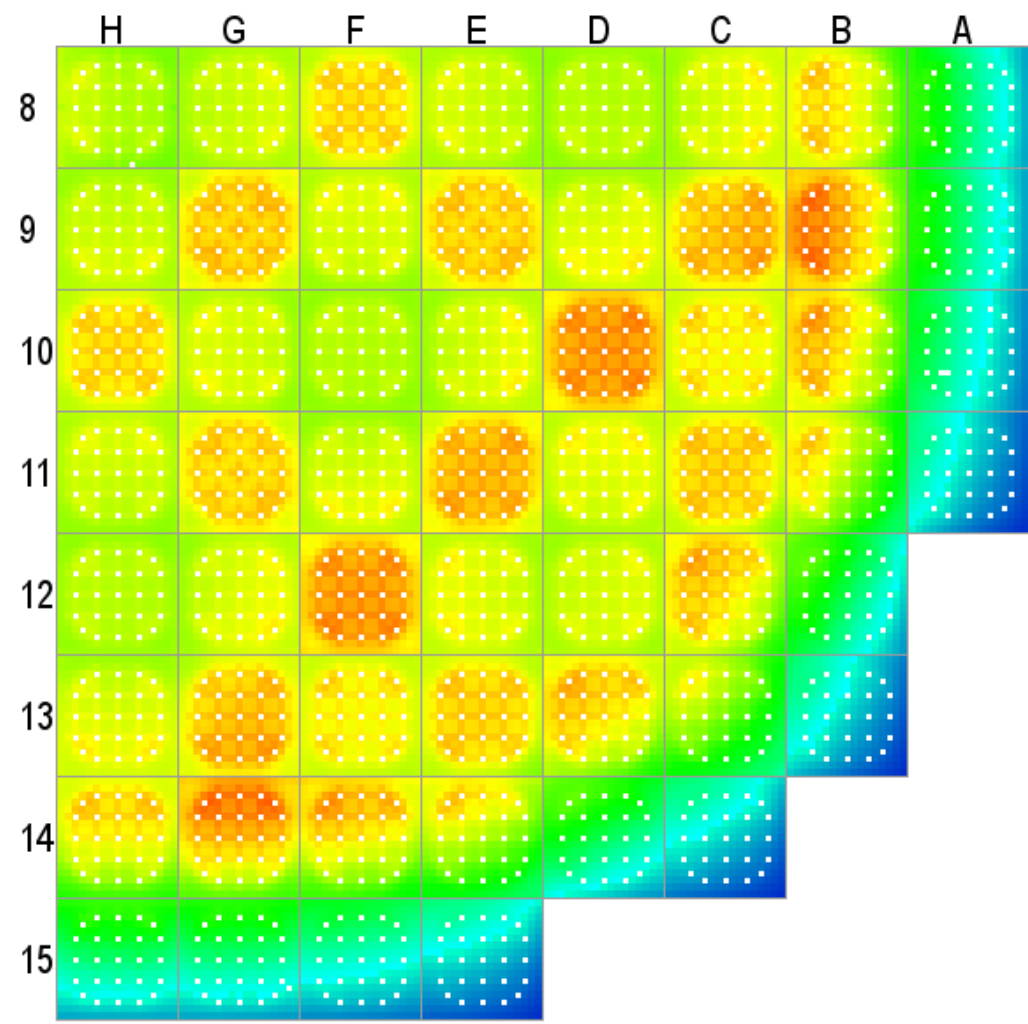

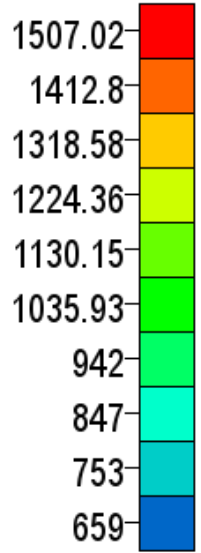

Fig. A.3.15. Cycle 3 maximum centerline fuel temperature (K) - 12.94 GWd/MT.

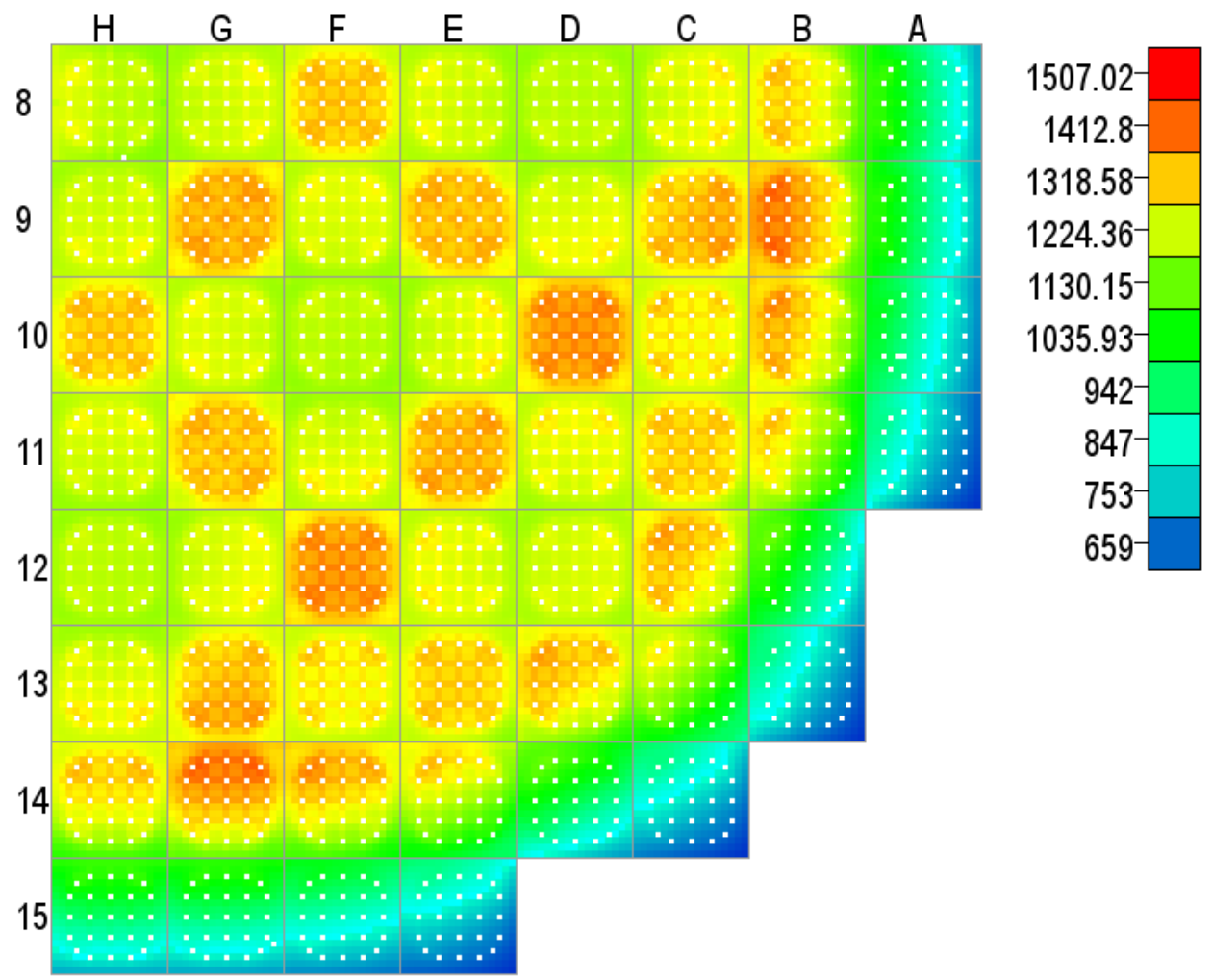

Fig. A.3.16. Cycle 3 maximum centerline fuel temperature $(\mathrm{K})-14.56 \mathrm{GWd} / \mathrm{MT}$. 

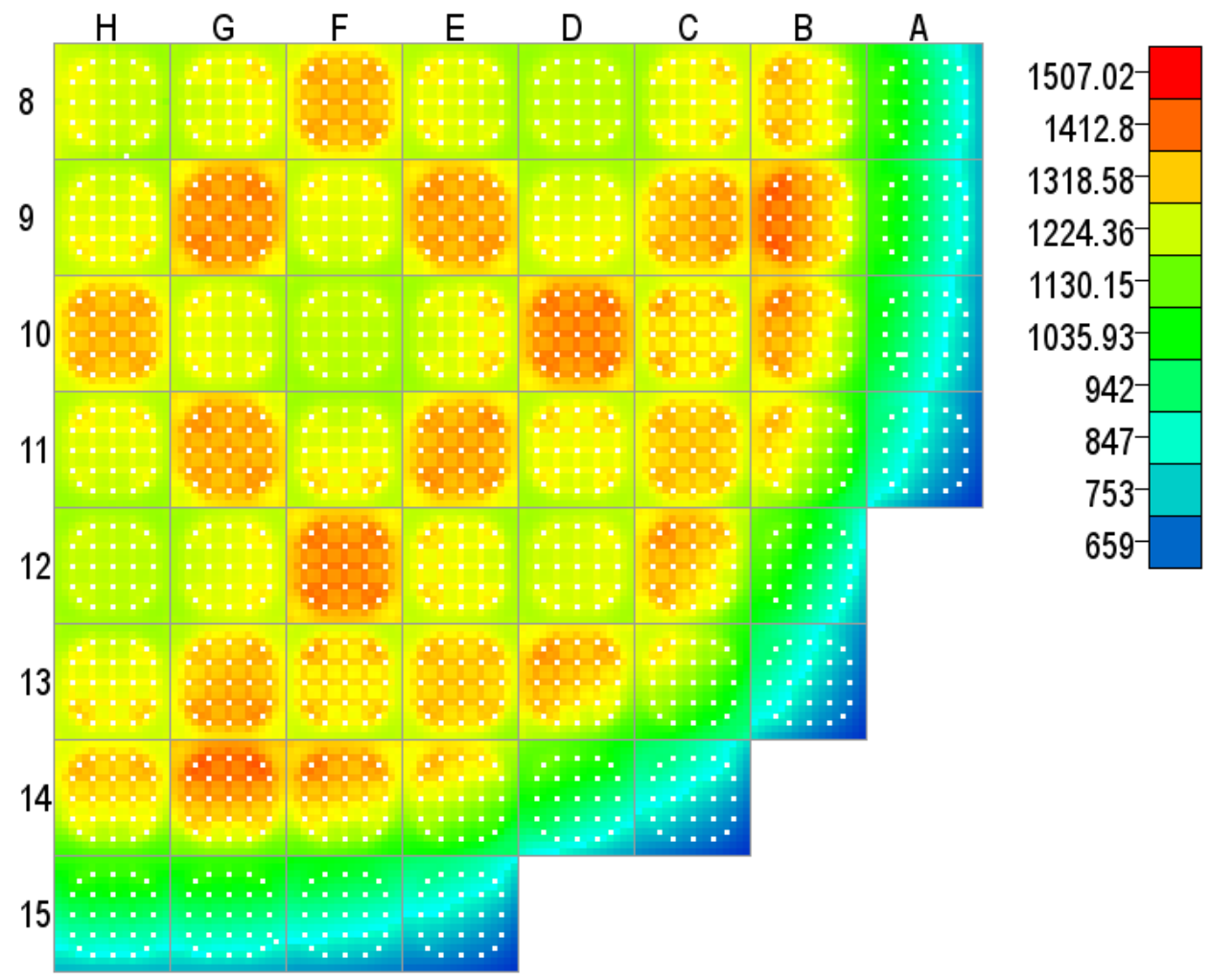

Fig. A.3.17. Cycle 3 maximum centerline fuel temperature $(\mathrm{K})-16.70 \mathrm{GWd} / \mathrm{MT}$.

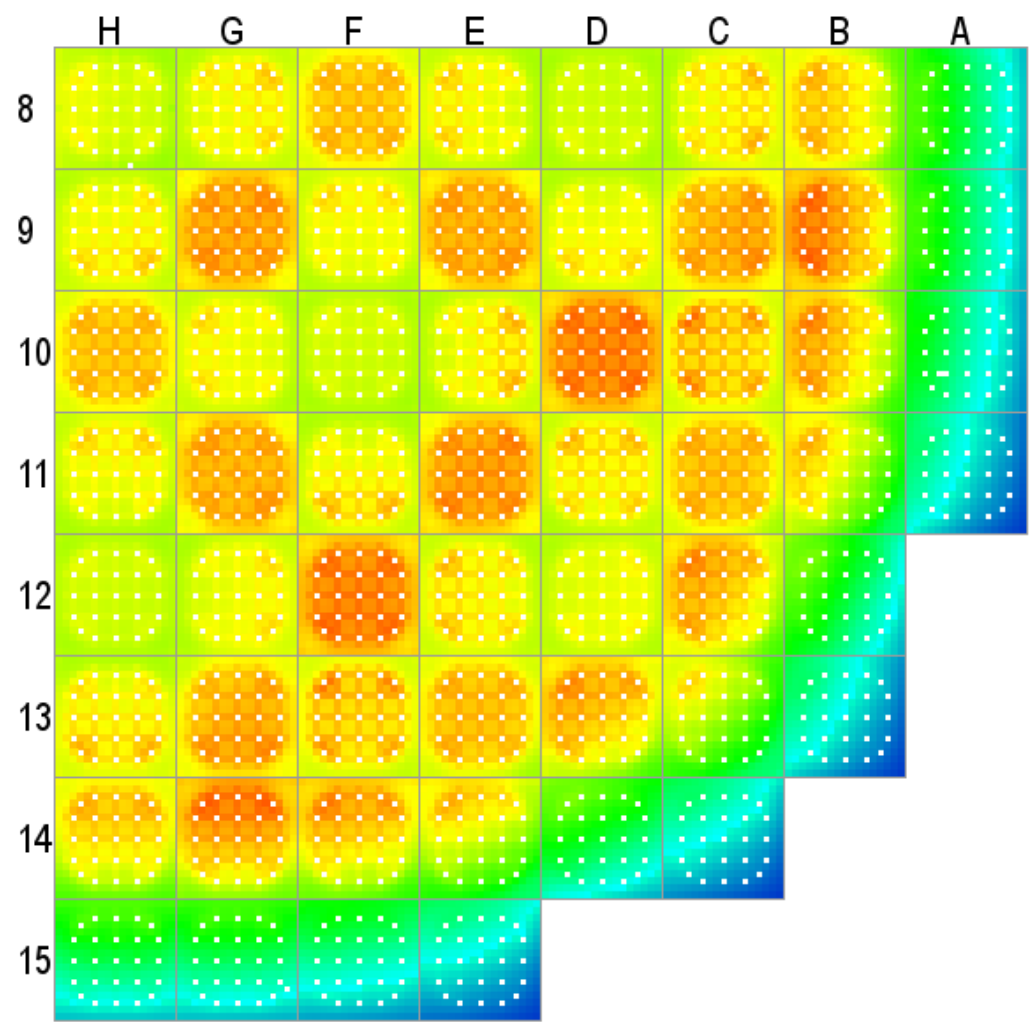

1507.02

1412.8

1318.58

1224.36

1130.15

1035.93

$942-$

847

753

659

Fig. A.3.18. Cycle 3 maximum centerline fuel temperature $(\mathrm{K})-18.14$ GWd/MT. 

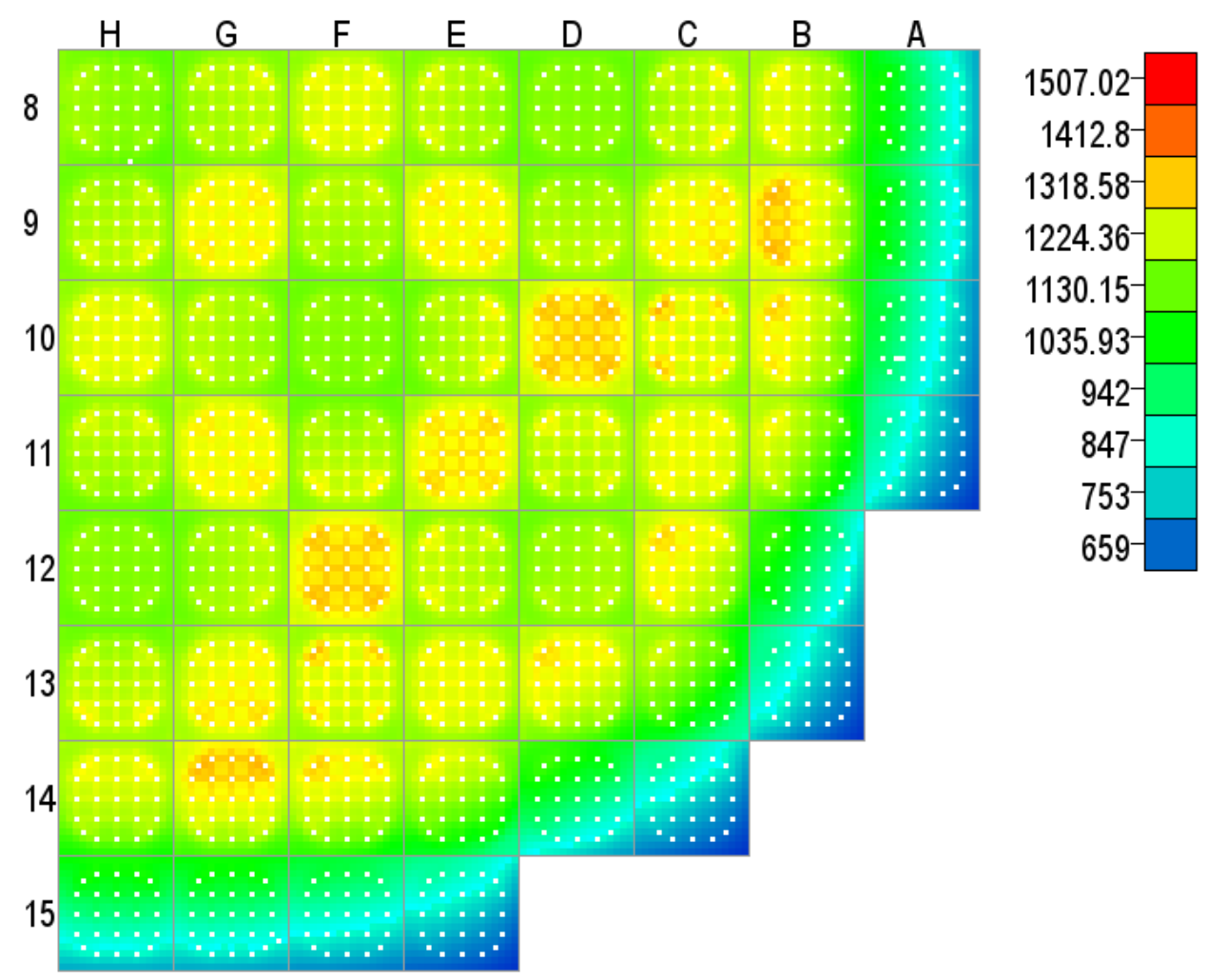

Fig. A.3.19. Cycle 3 maximum centerline fuel temperature $(\mathrm{K})-18.79$ GWd/MT.

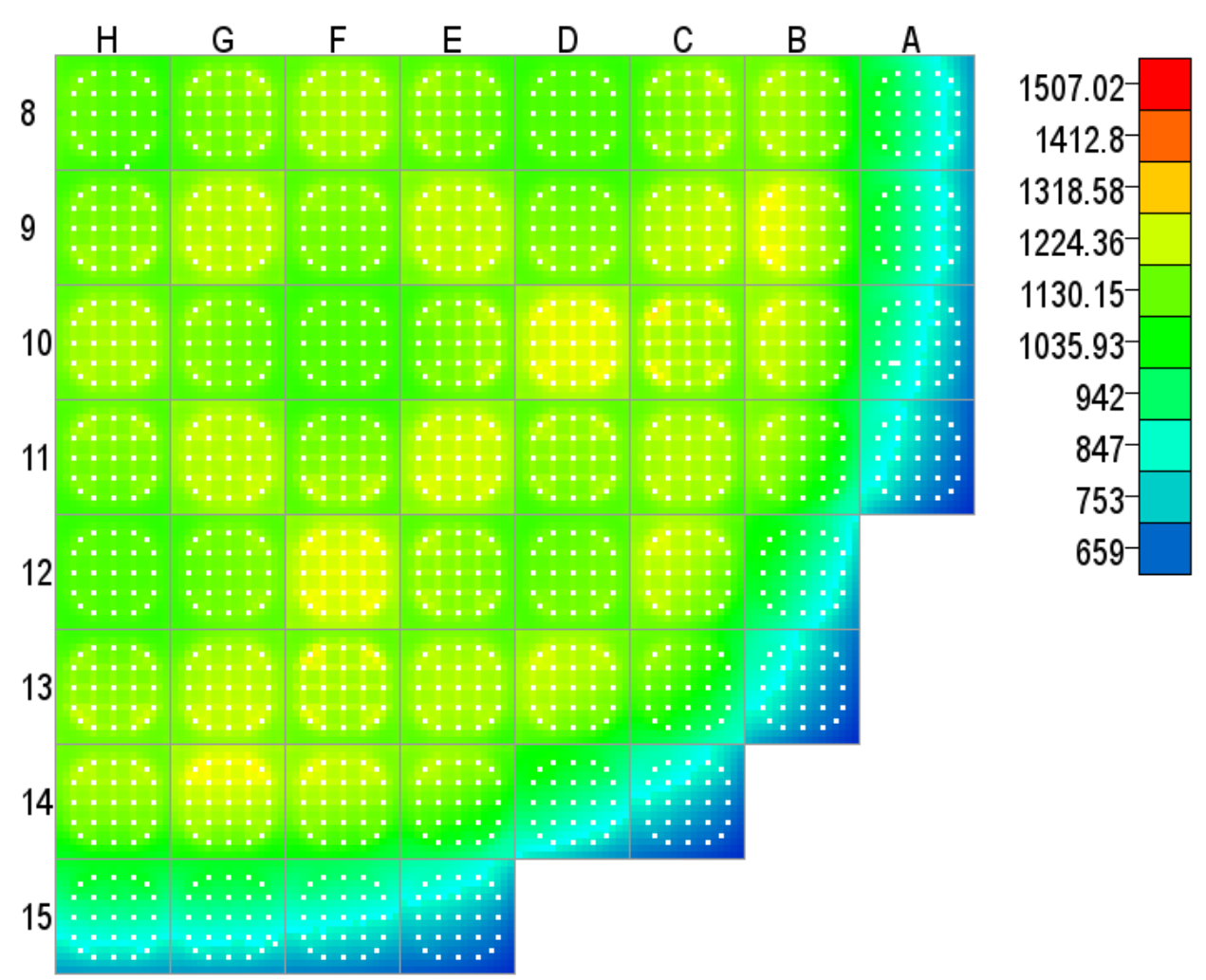

Fig. A.3.20. Cycle 3 maximum centerline fuel temperature (K) - 19.32 GWd/MT. 


\section{A.3.2. Minimum Gap Thickness}

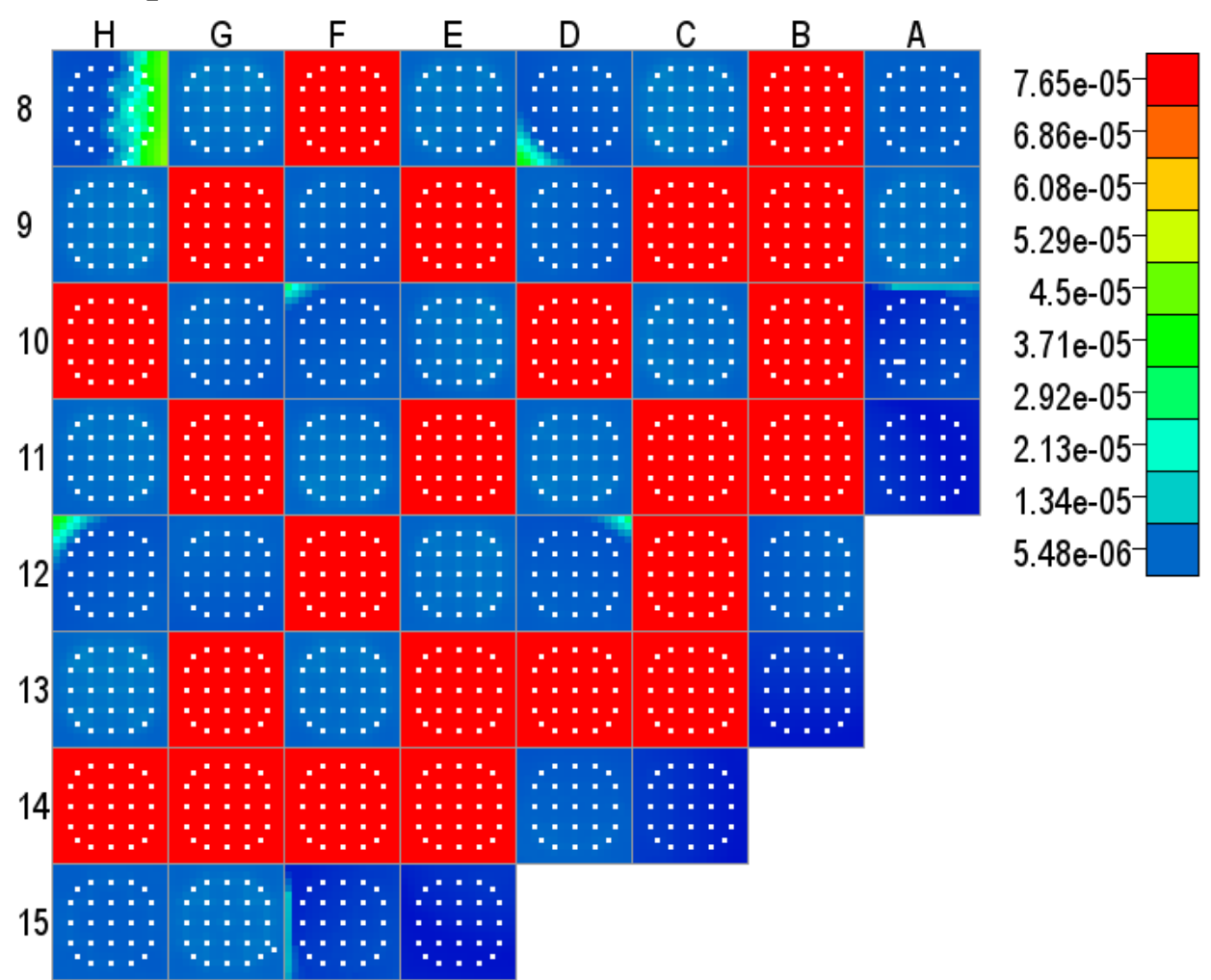

Fig. A.3.21. Cycle 3 minimum gap thickness (m) - 0.00 GWd/MT.

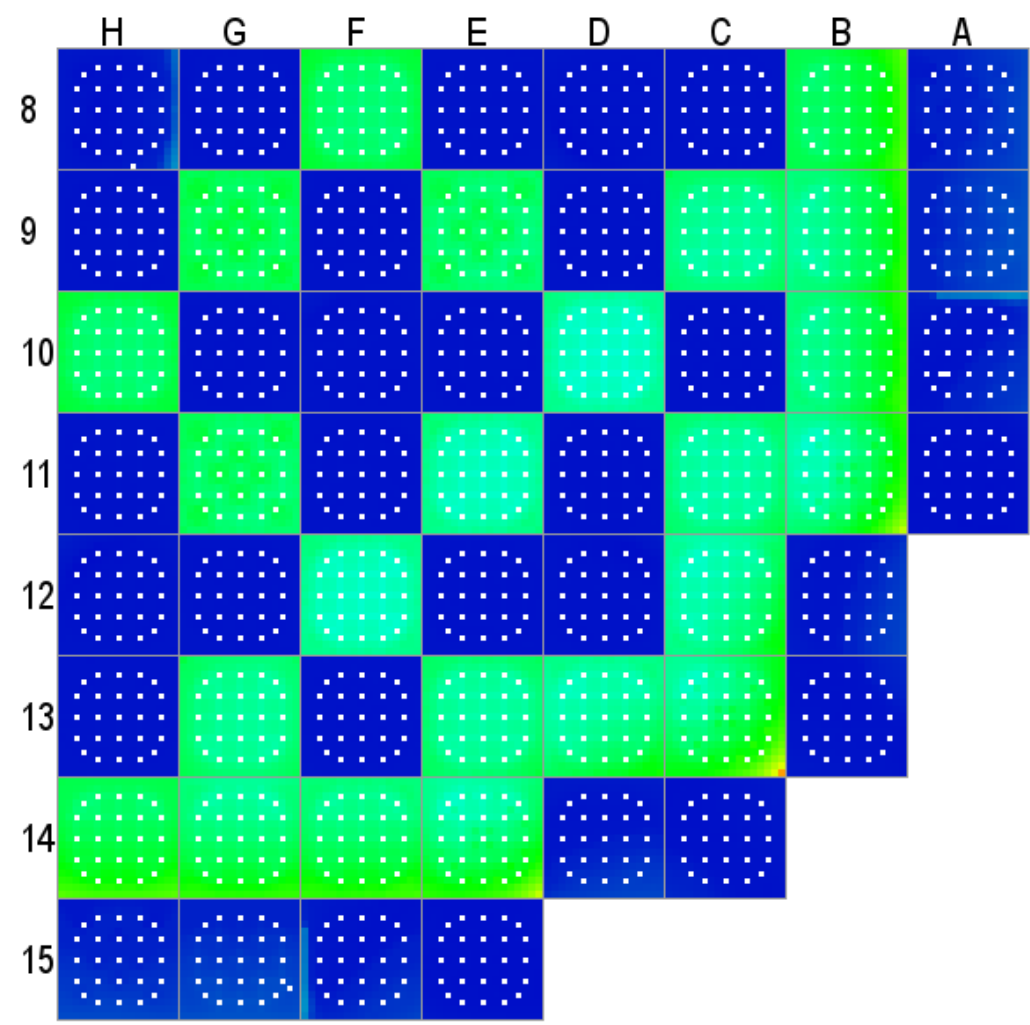

$7.65 \mathrm{e}-05$
$6.86 \mathrm{e}-05$
$6.08 \mathrm{e}-05$
$5.29 \mathrm{e}-05$
$4.5 \mathrm{e}-05$
$3.71 \mathrm{e}-05$
$2.92 \mathrm{e}-05$
$2.13 \mathrm{e}-05$
$1.34 \mathrm{e}-05$
$5.48 \mathrm{e}-06$

Fig. A.3.22. Cycle 3 minimum gap thickness $(\mathrm{m})-0.20 \mathrm{GWd} / \mathrm{MT}$. 


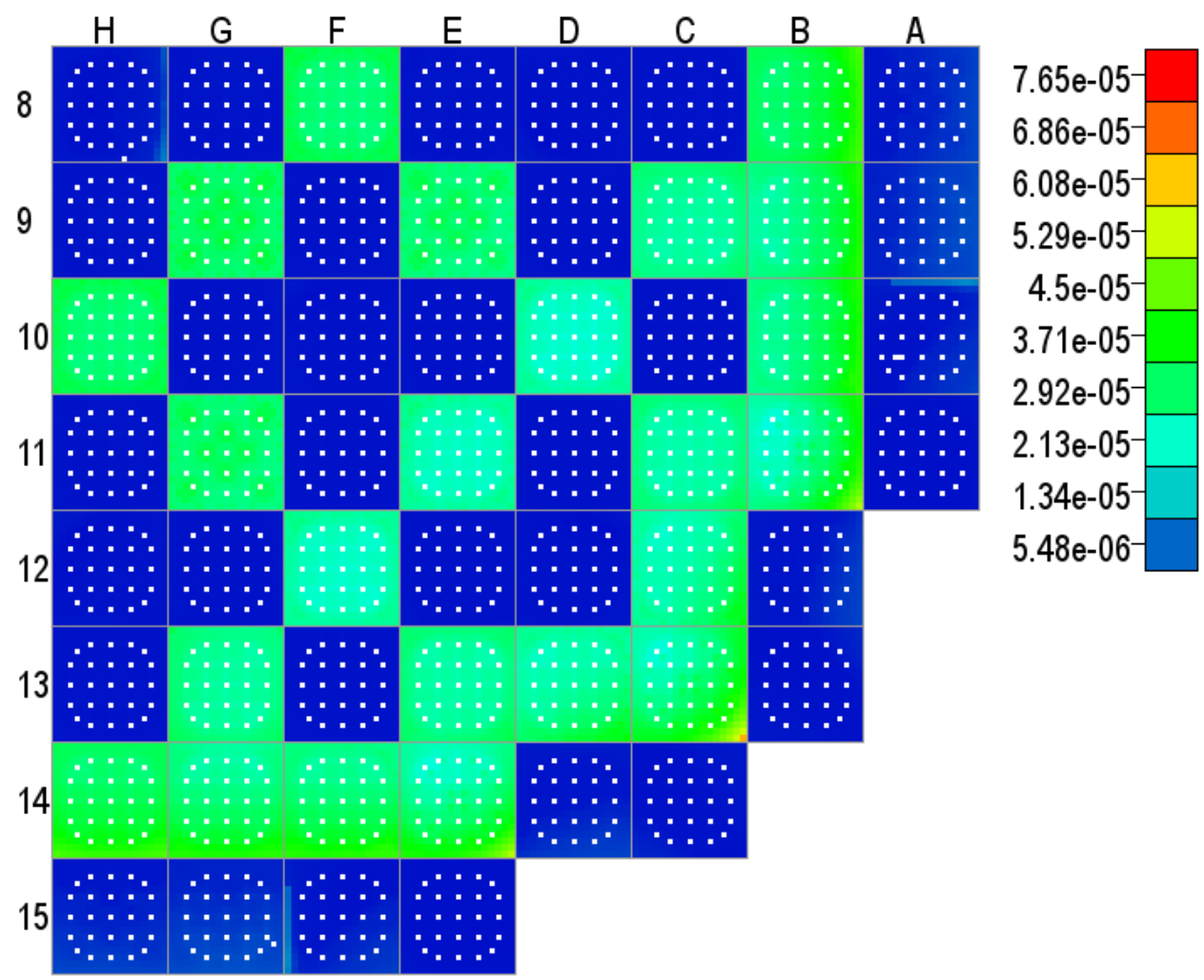

Fig. A.3.23. Cycle 3 minimum gap thickness (m) - 0.69 GWd/MT.

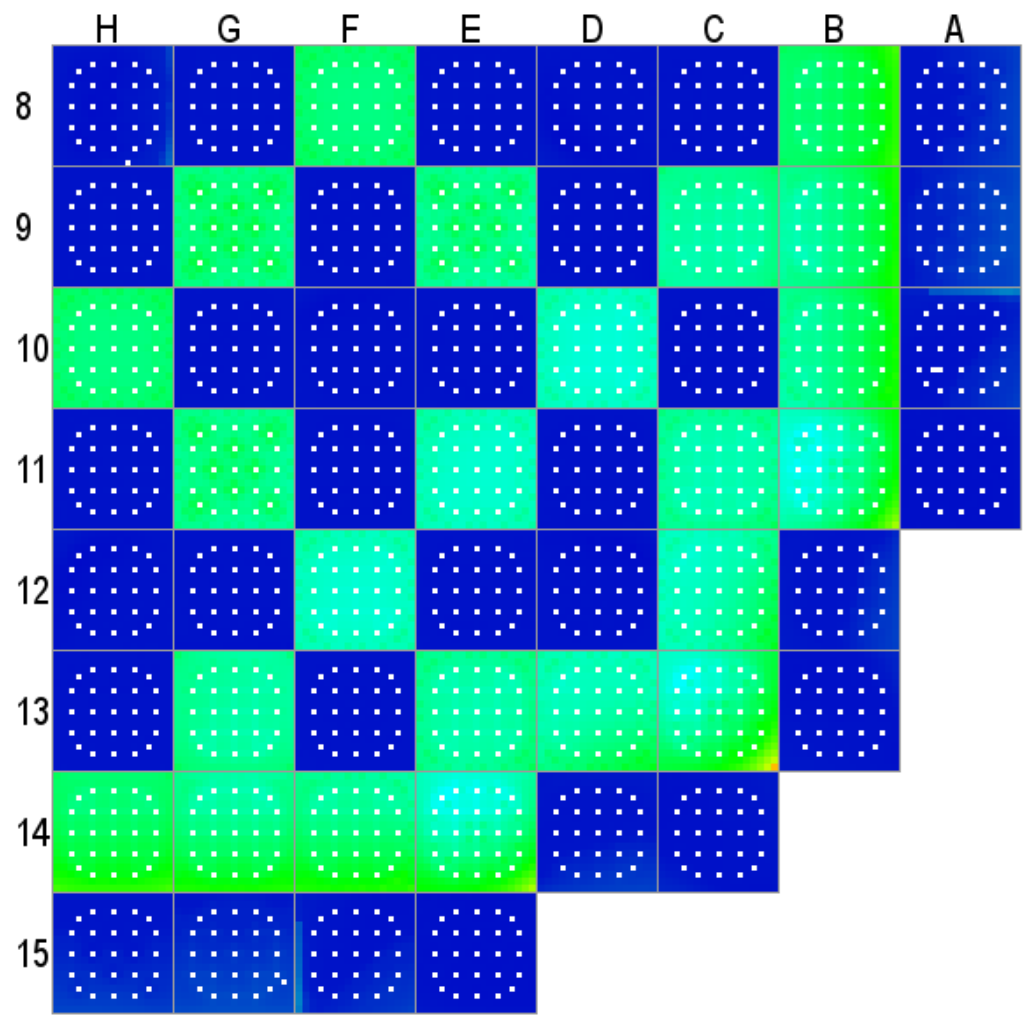

$7.65 \mathrm{e}-05$
$6.86 \mathrm{e}-05$
$6.08 \mathrm{e}-05$
$5.29 \mathrm{e}-05$
$4.5 \mathrm{e}-05$
$3.71 \mathrm{e}-05$
$2.92 \mathrm{e}-05$
$2.13 \mathrm{e}-05$
$1.34 \mathrm{e}-05$
$5.48 \mathrm{e}-05$

Fig. A.3.24. Cycle 3 minimum gap thickness $(\mathrm{m})-1.17$ GWd/MT. 


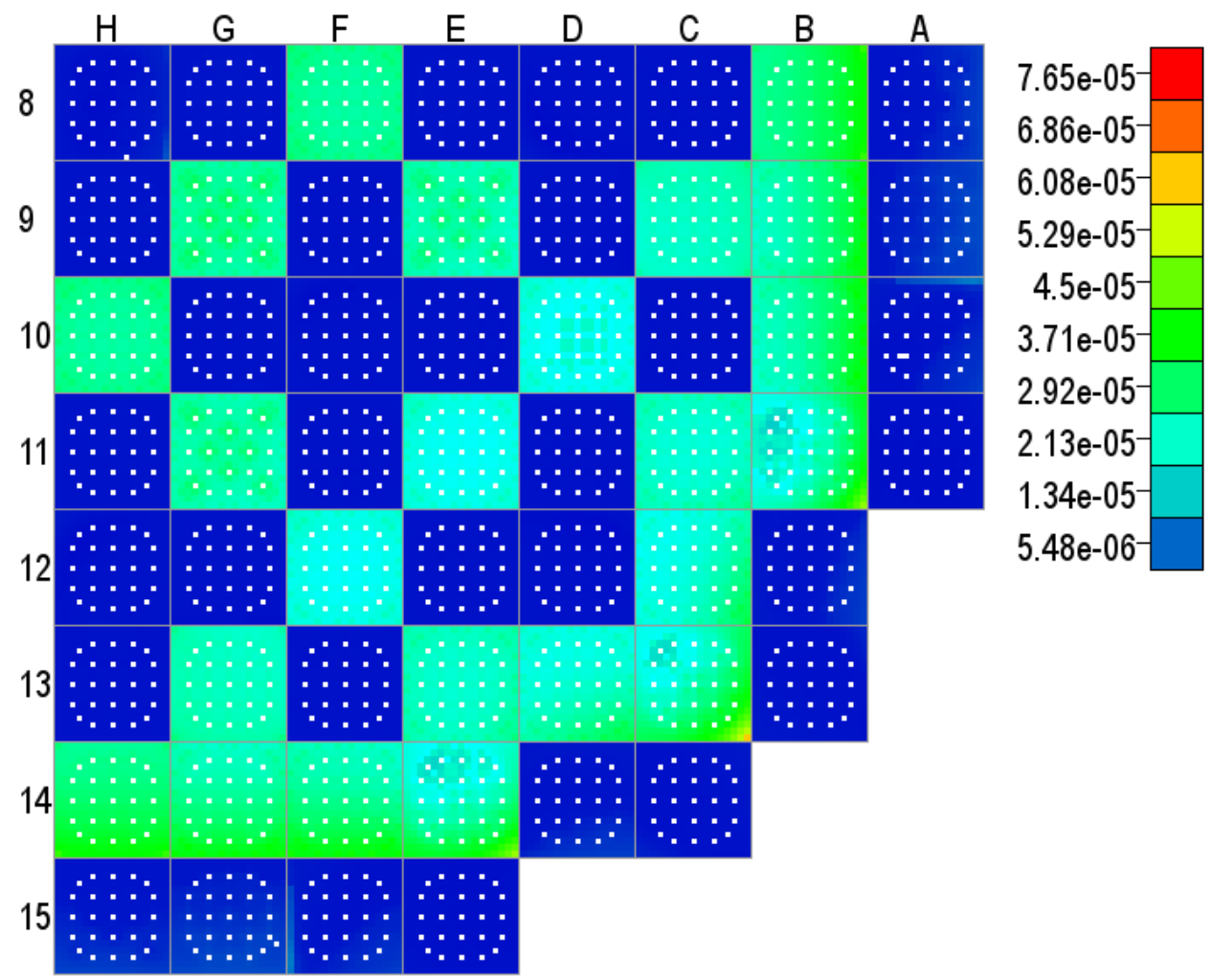

Fig. A.3.25. Cycle 3 minimum gap thickness (m) - 1.70 GWd/MT.

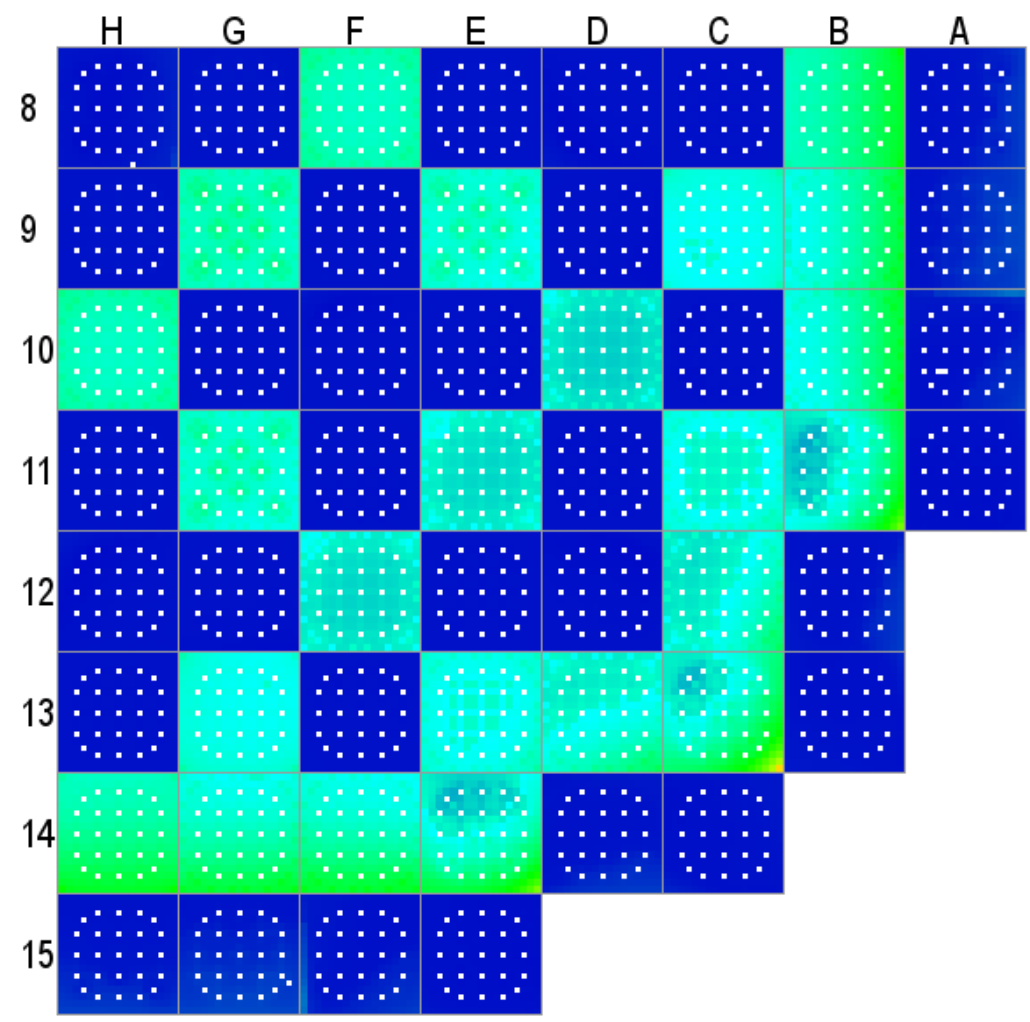

$7.65 \mathrm{e}-05-$
$6.86 \mathrm{e}-05$
$6.08 \mathrm{e}-05$
$5.29 \mathrm{e}-05$
$4.5 \mathrm{e}-05$
$3.71 \mathrm{e}-05$
$2.92 \mathrm{e}-05$
$2.13 \mathrm{e}-05$
$1.34 \mathrm{e}-05$
$5.48 \mathrm{e}-05-$

Fig. A.3.26. Cycle 3 minimum gap thickness (m) - 2.24 GWd/MT. 


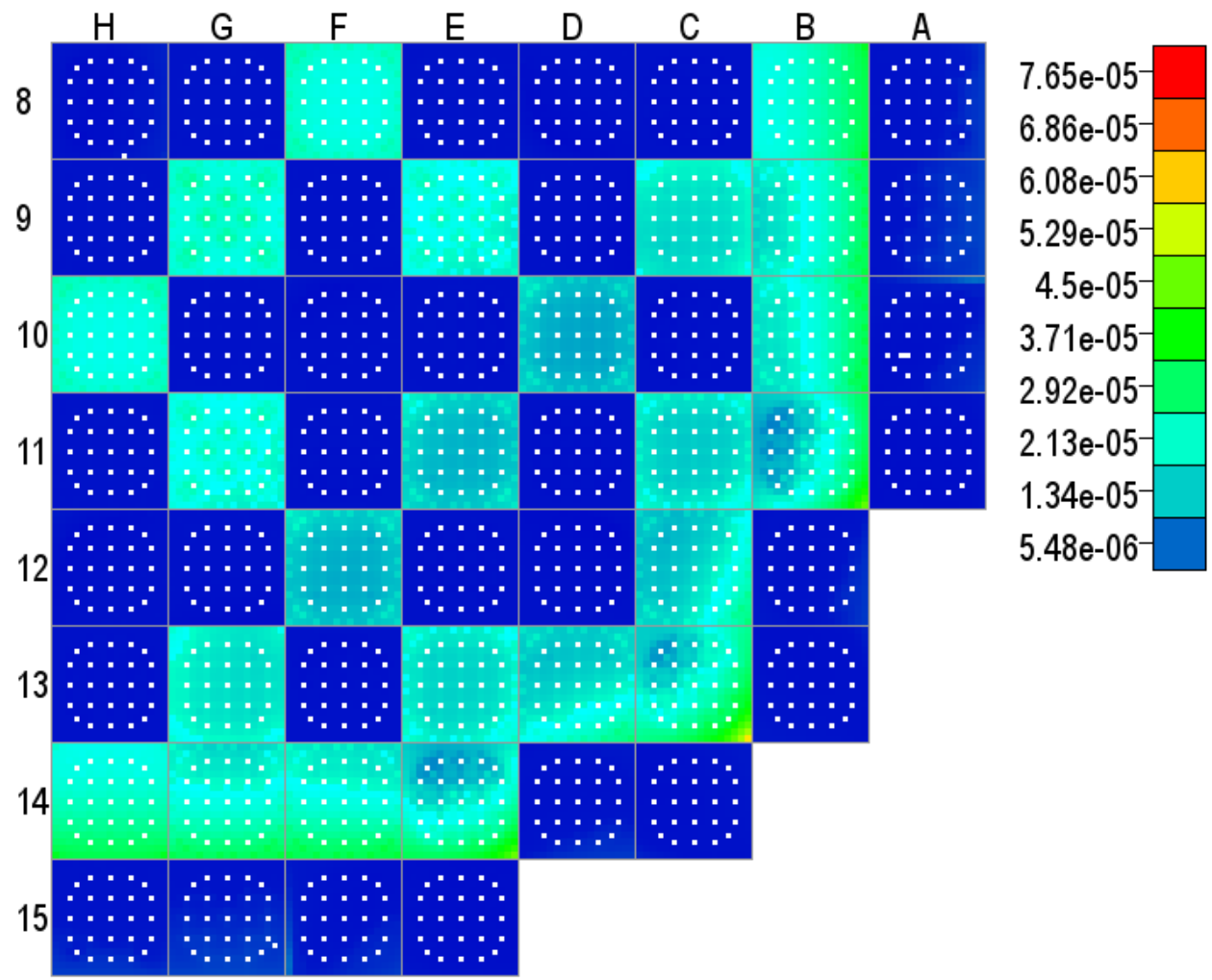

Fig. A.3.27. Cycle 3 minimum gap thickness (m) - 2.77 GWd/MT.

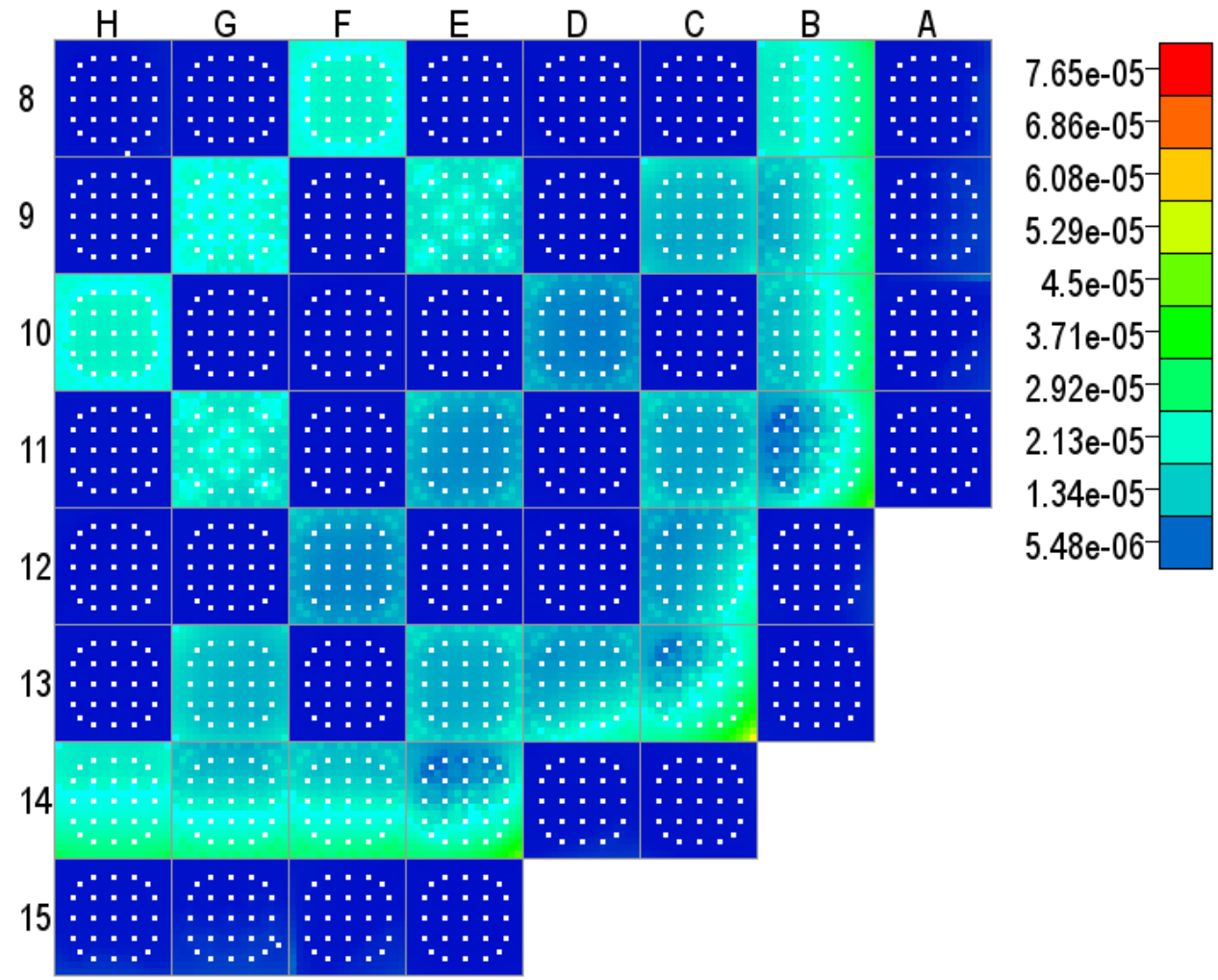

Fig. A.3.28. Cycle 3 minimum gap thickness (m) - 3.30 GWd/MT. 


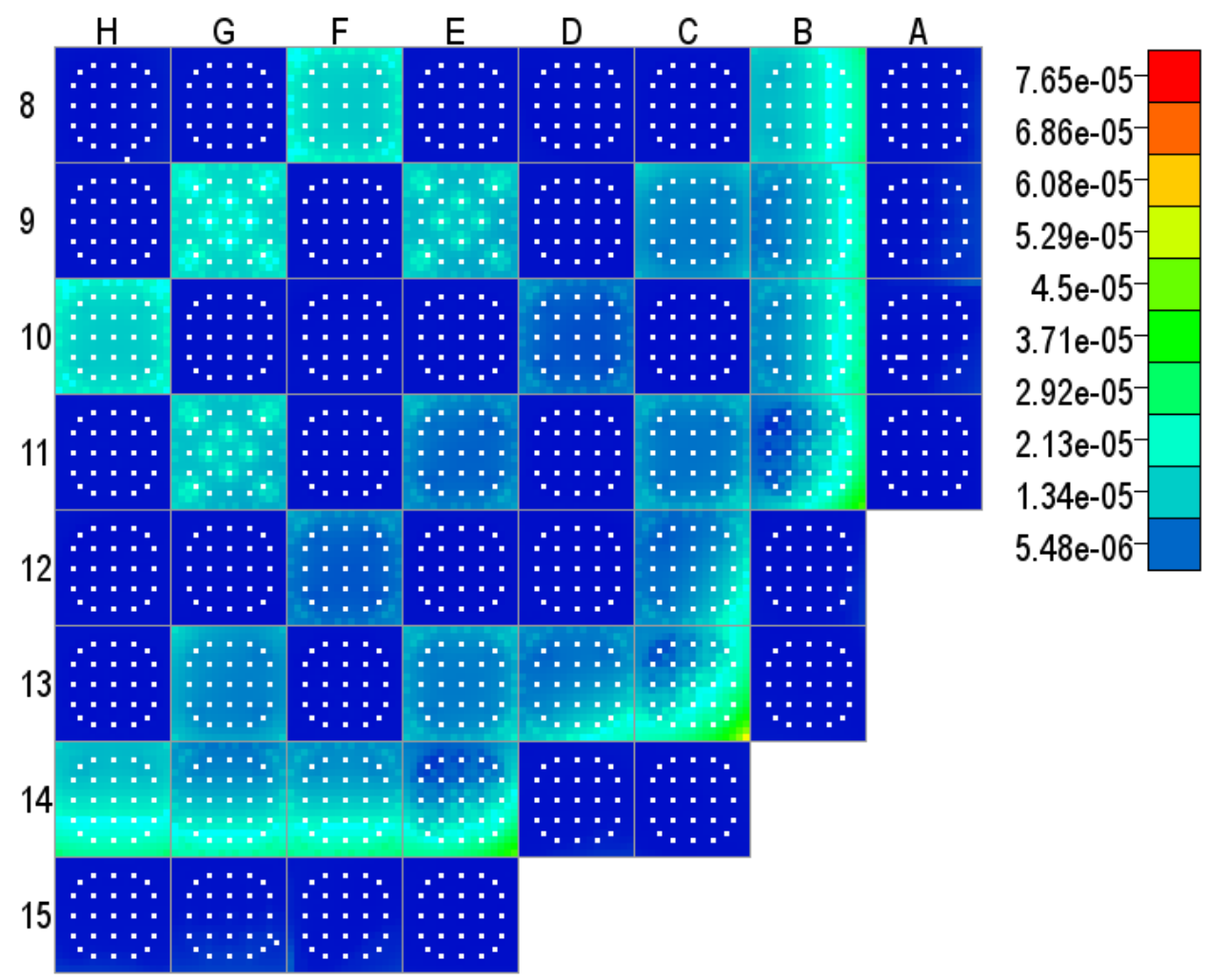

Fig. A.3.29. Cycle 3 minimum gap thickness $(\mathrm{m})-3.96 \mathrm{GWd} / \mathrm{MT}$.

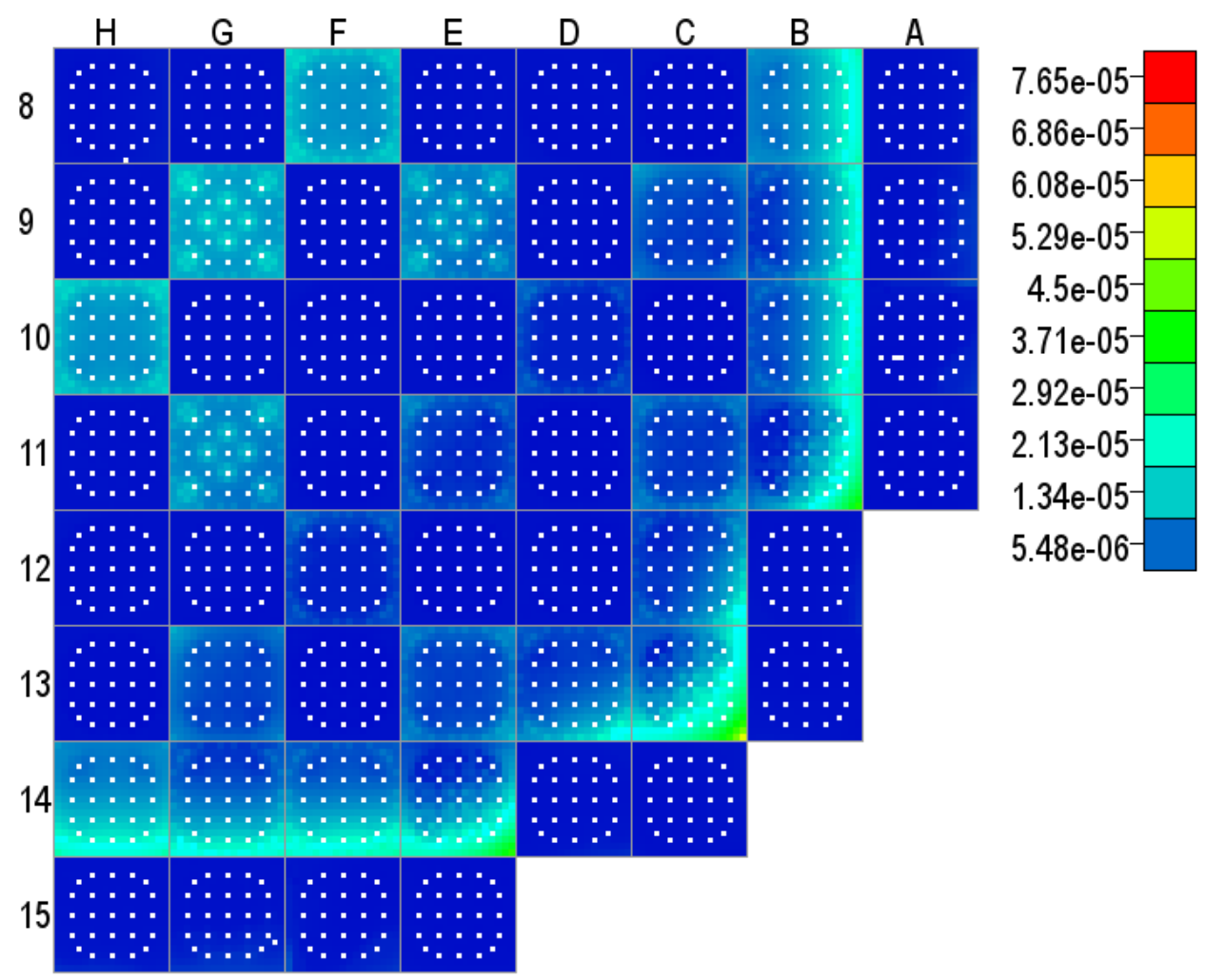

Fig. A.3.30. Cycle 3 minimum gap thickness (m) -4.91 GWd/MT. 


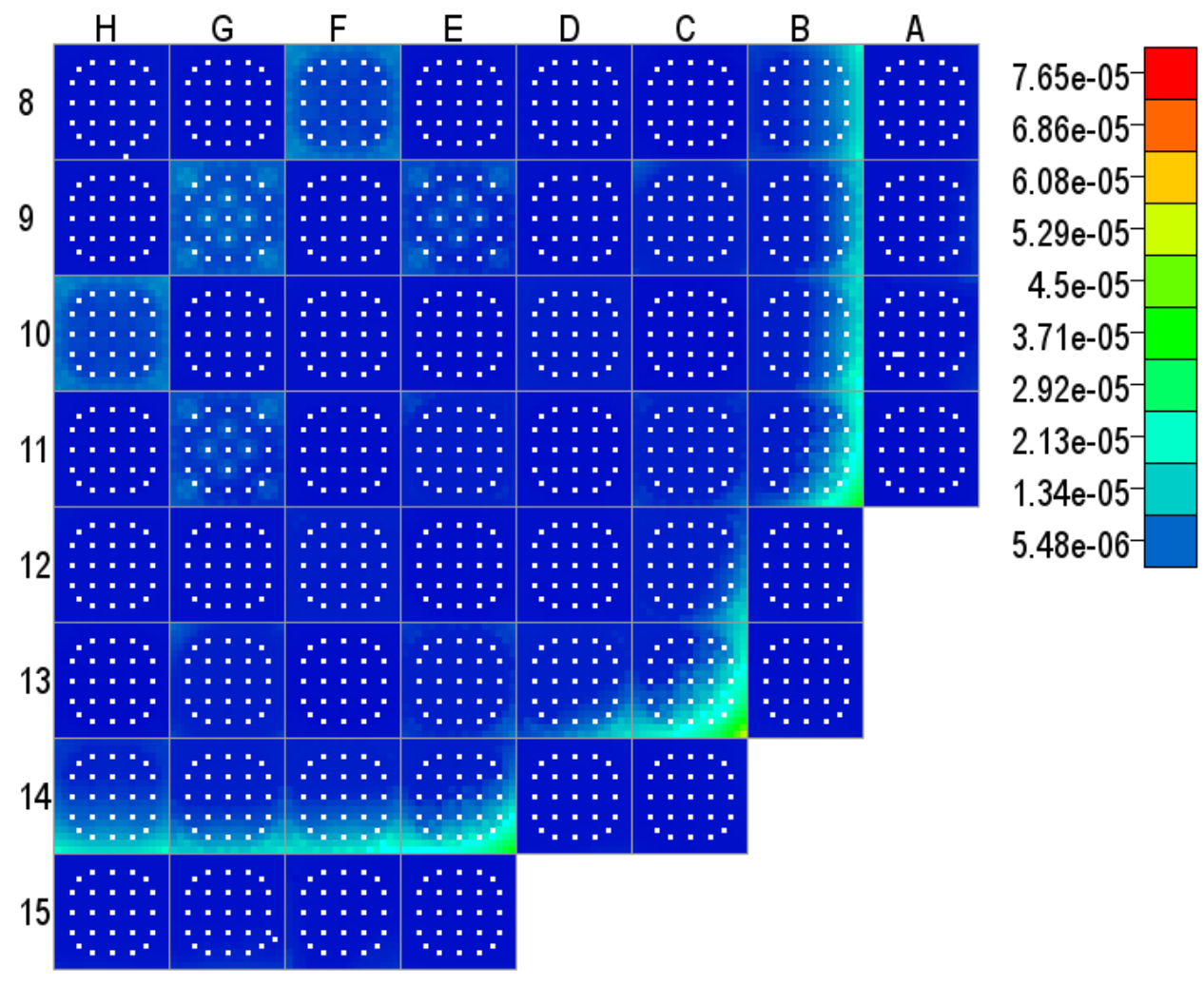

Fig. A.3.31. Cycle 3 minimum gap thickness (m) - 6.44 GWd/MT.

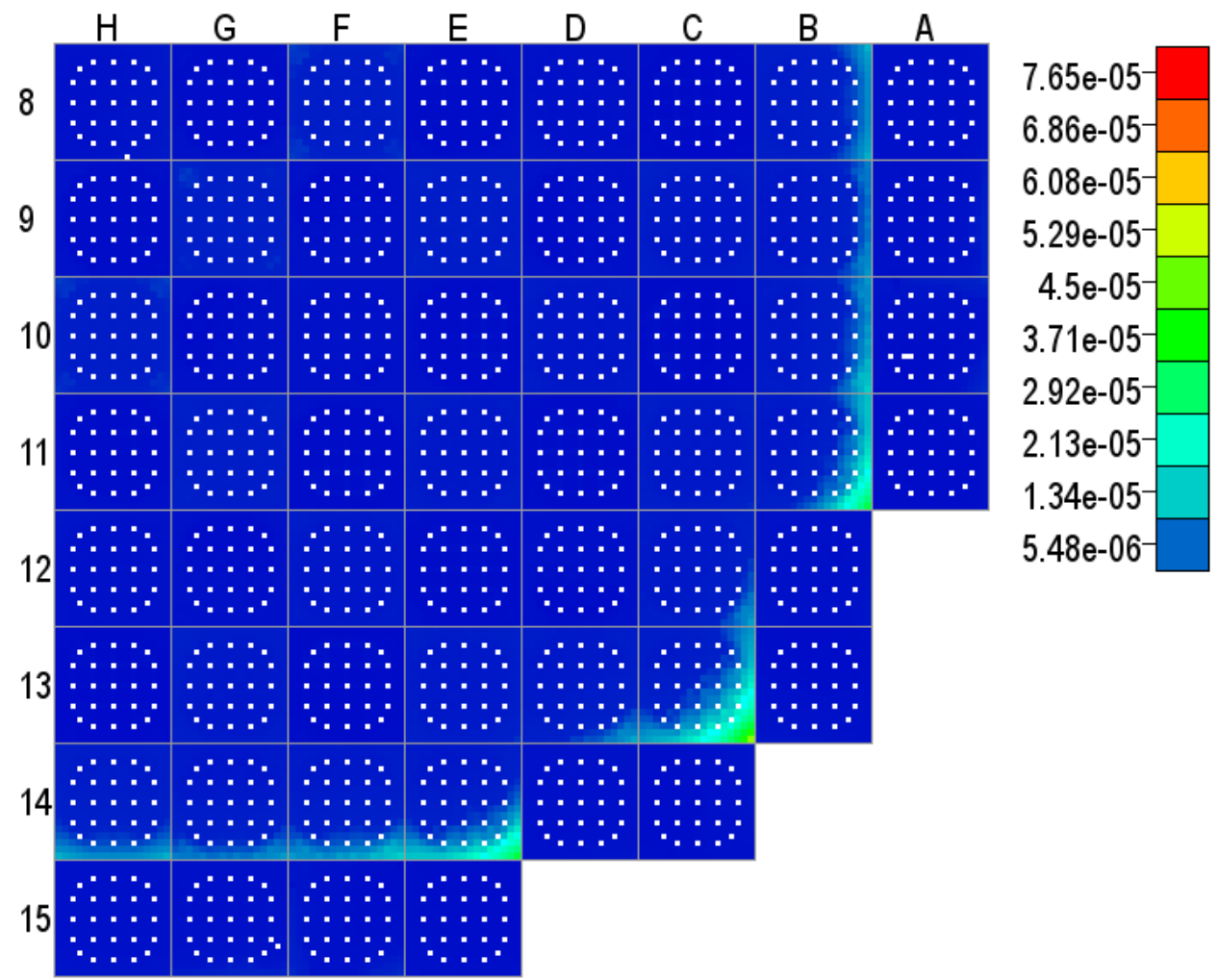

Fig. A.3.32. Cycle 3 minimum gap thickness (m) $-8.13 \mathrm{GWd} / \mathrm{MT}$. 


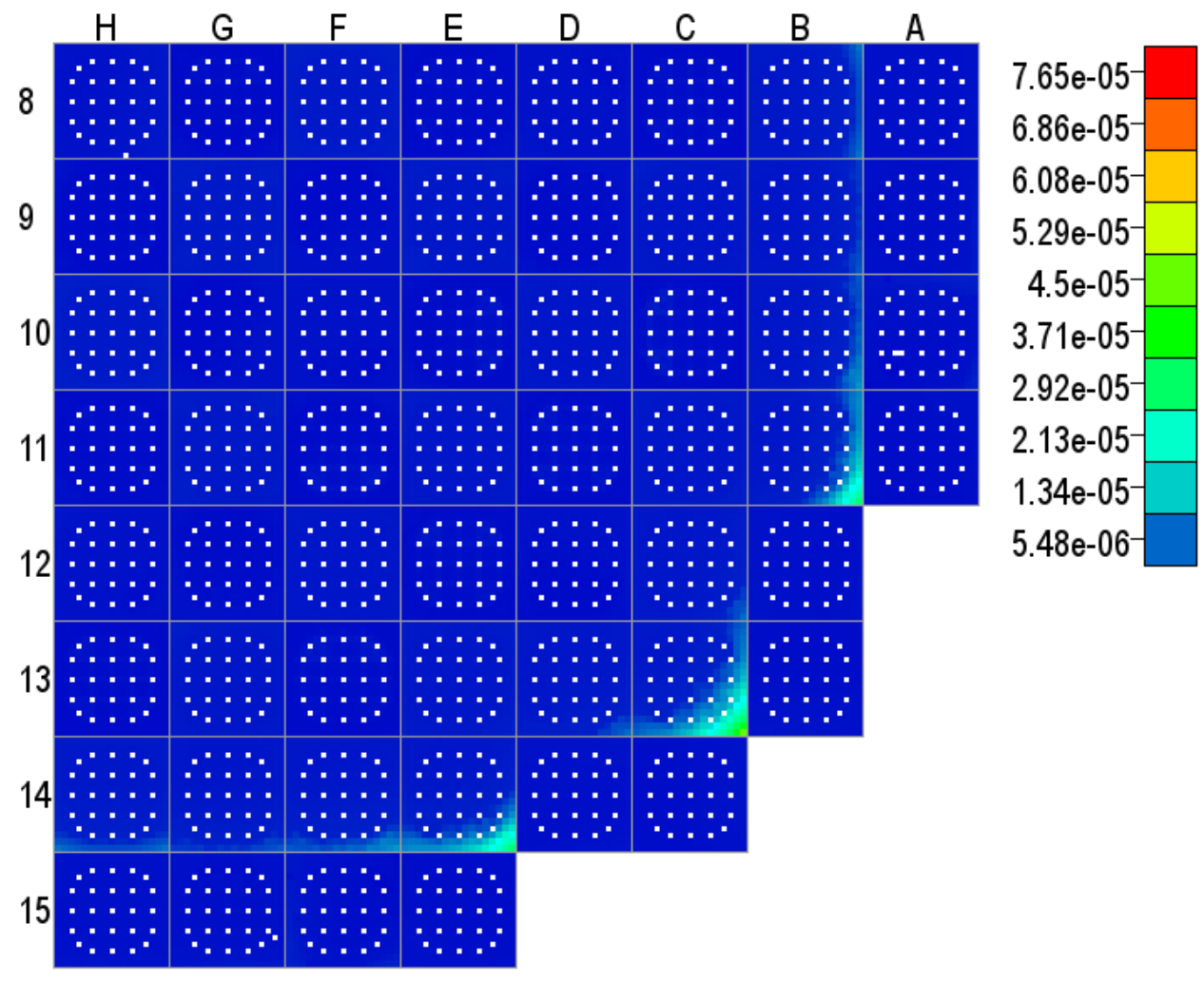

Fig. A.3.33. Cycle 3 minimum gap thickness (m) - 9.72 GWd/MT.

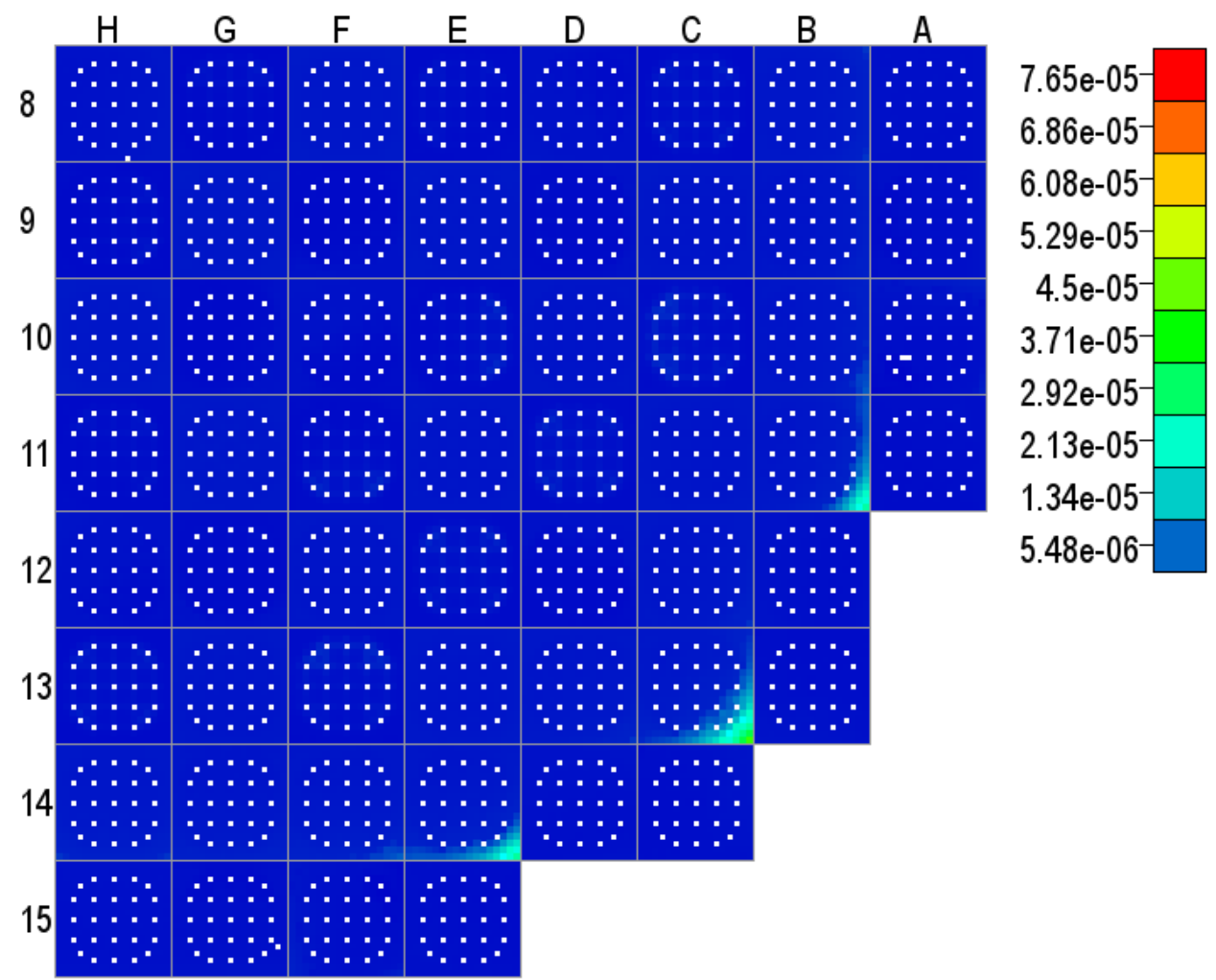

Fig. A.3.34. Cycle 3 minimum gap thickness (m) - 11.35 GWd/MT. 


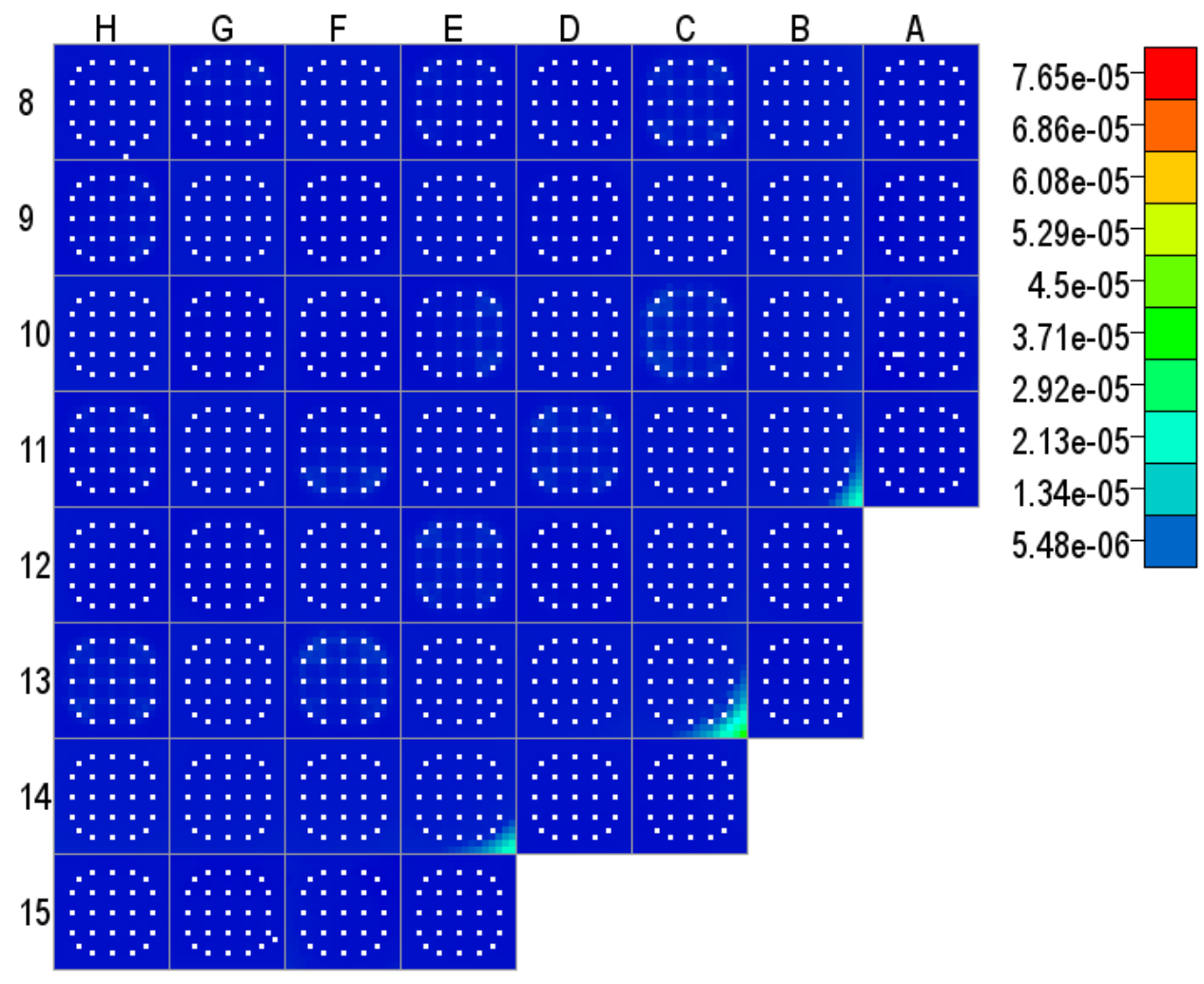

Fig. A.3.35. Cycle 3 minimum gap thickness (m) - 12.94 GWd/MT.

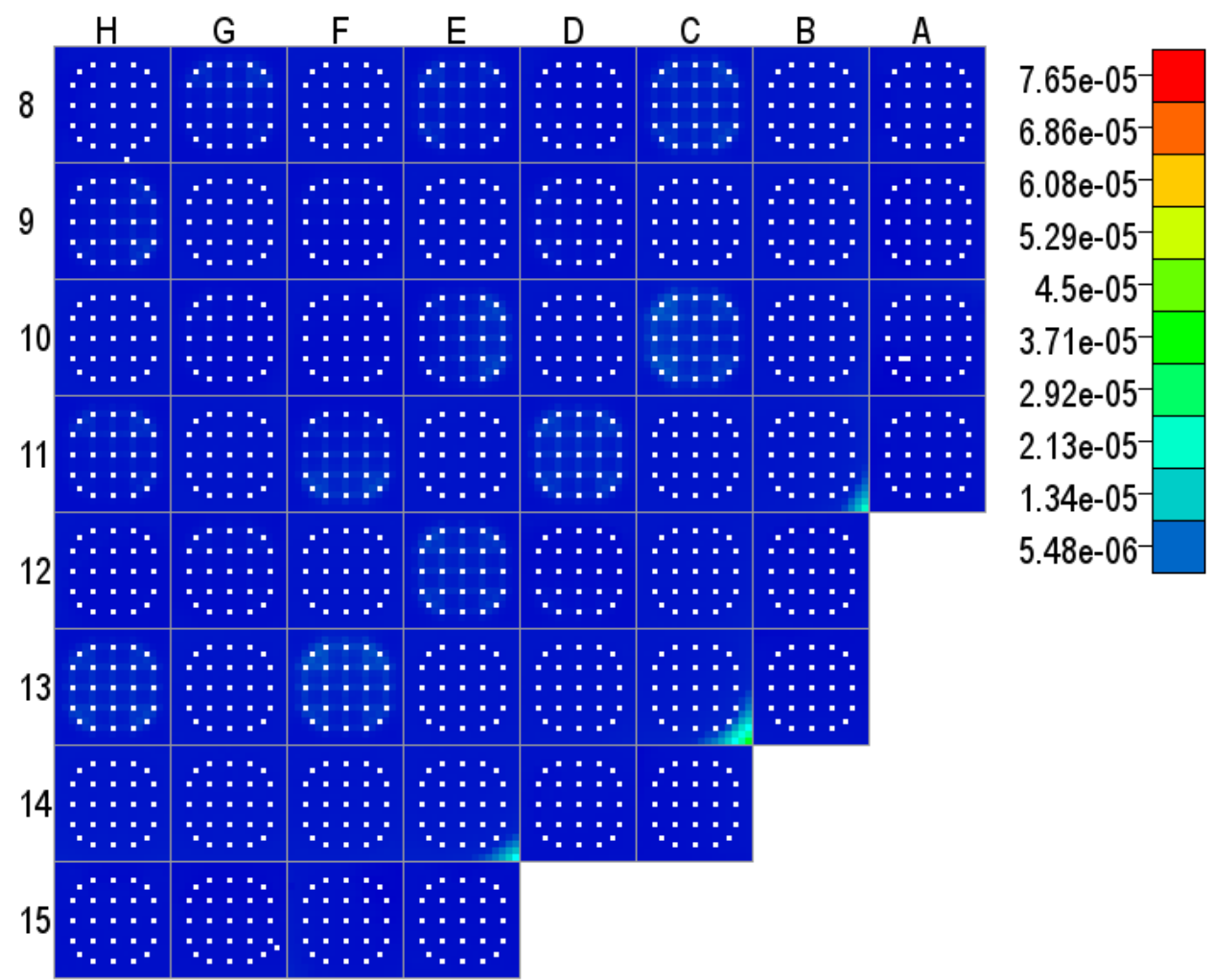

Fig. A.3.36. Cycle 3 minimum gap thickness (m) - 14.56 GWd/MT. 


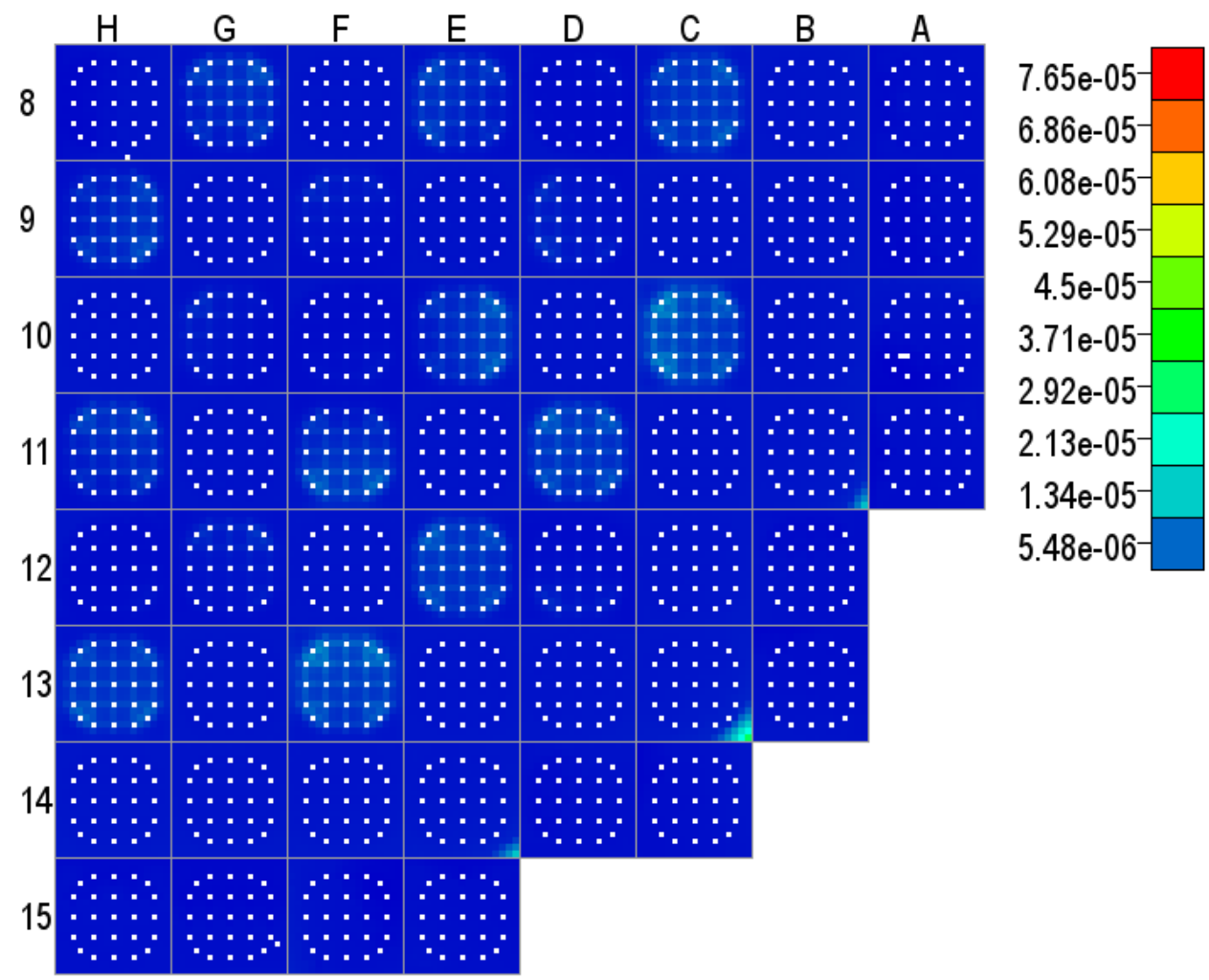

Fig. A.3.37. Cycle 3 minimum gap thickness (m) - 16.70 GWd/MT.

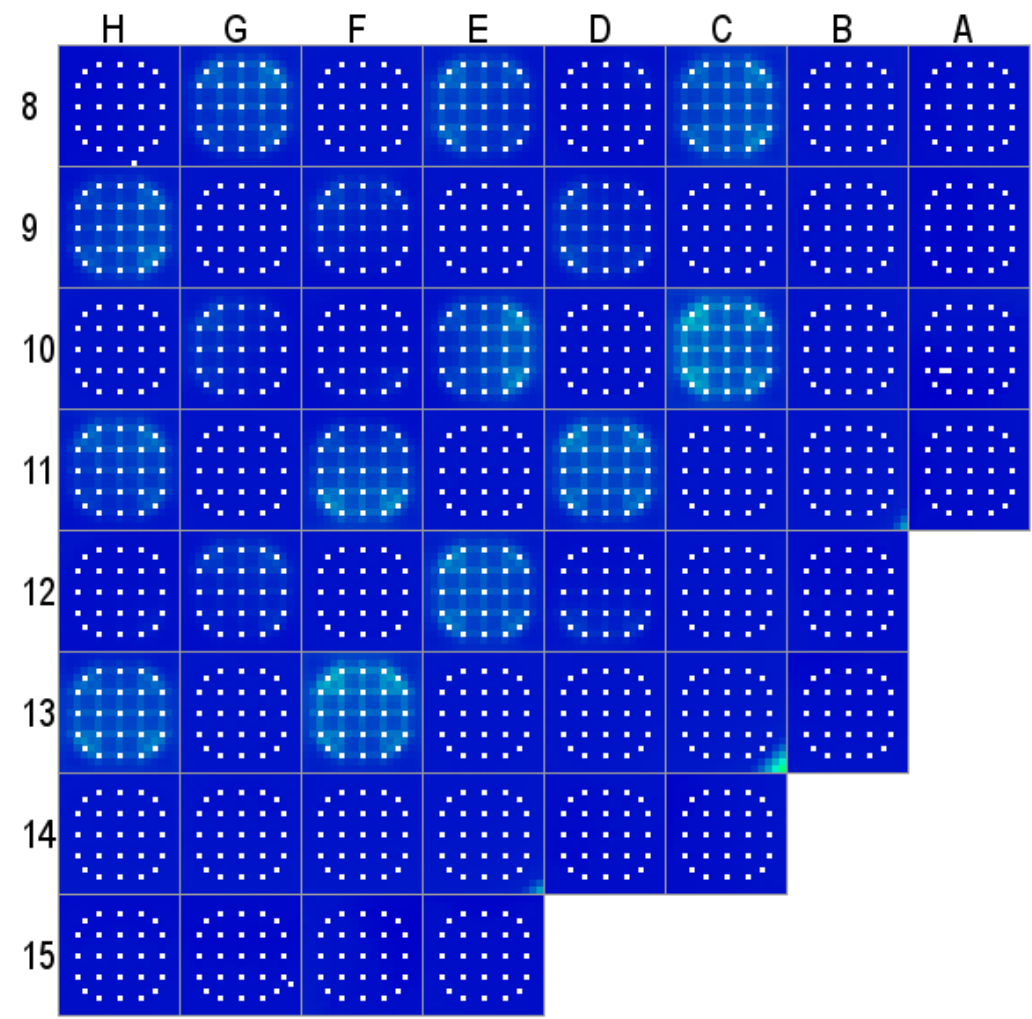

$7.65 \mathrm{e}-05$
$6.86 \mathrm{e}-05$
$6.08 \mathrm{e}-05$
$5.29 \mathrm{e}-05$
$4.5 \mathrm{e}-05$
$3.71 \mathrm{e}-05$
$2.92 \mathrm{e}-05$
$2.13 \mathrm{e}-05$
$1.34 \mathrm{e}-05$
$5.48 \mathrm{e}-06$

Fig. A.3.38. Cycle 3 minimum gap thickness $(\mathrm{m})$ - 18.14 GWd/MT. 


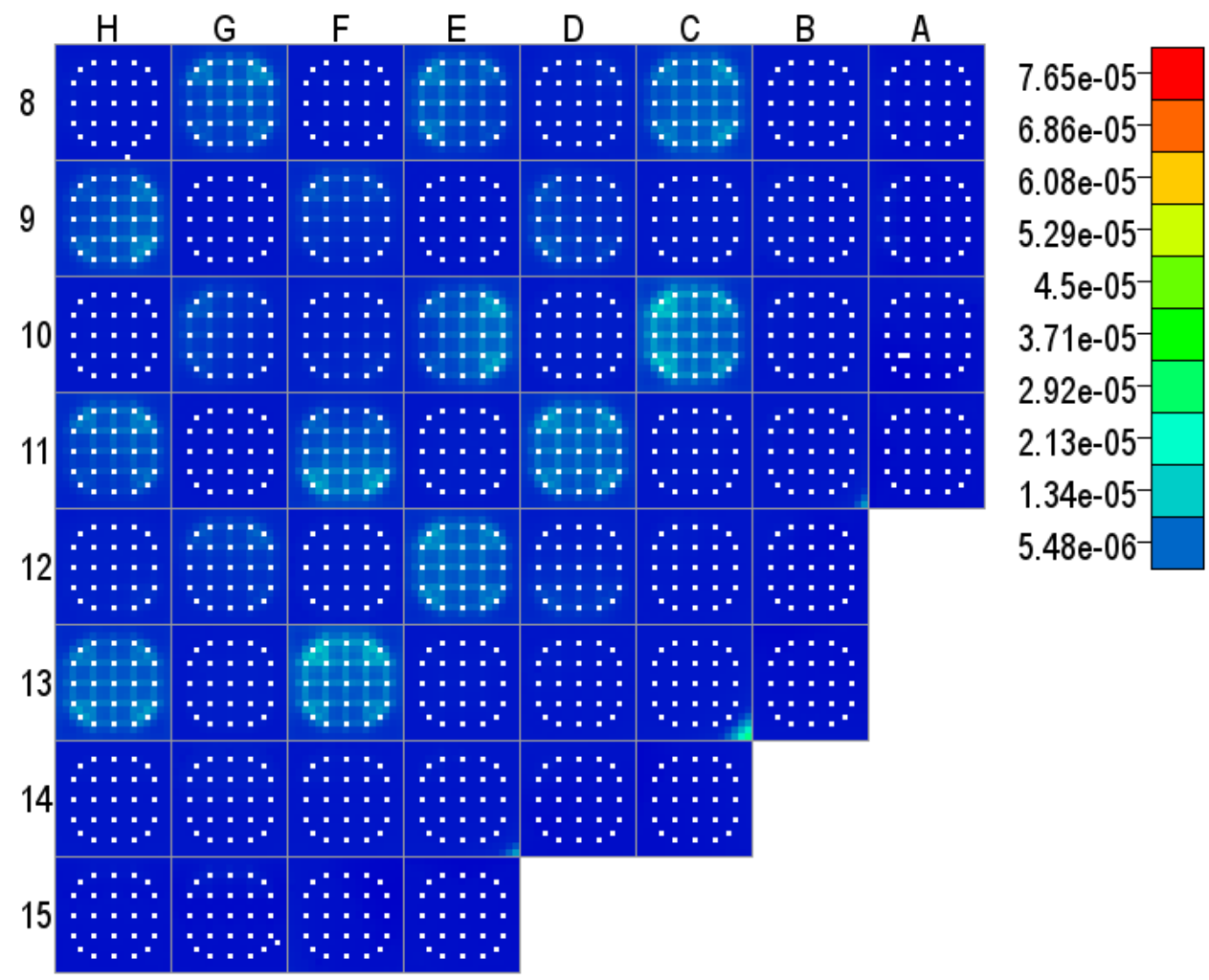

Fig. A.3.39. Cycle 3 minimum gap thickness $(m)-18.79$ GWd/MT.

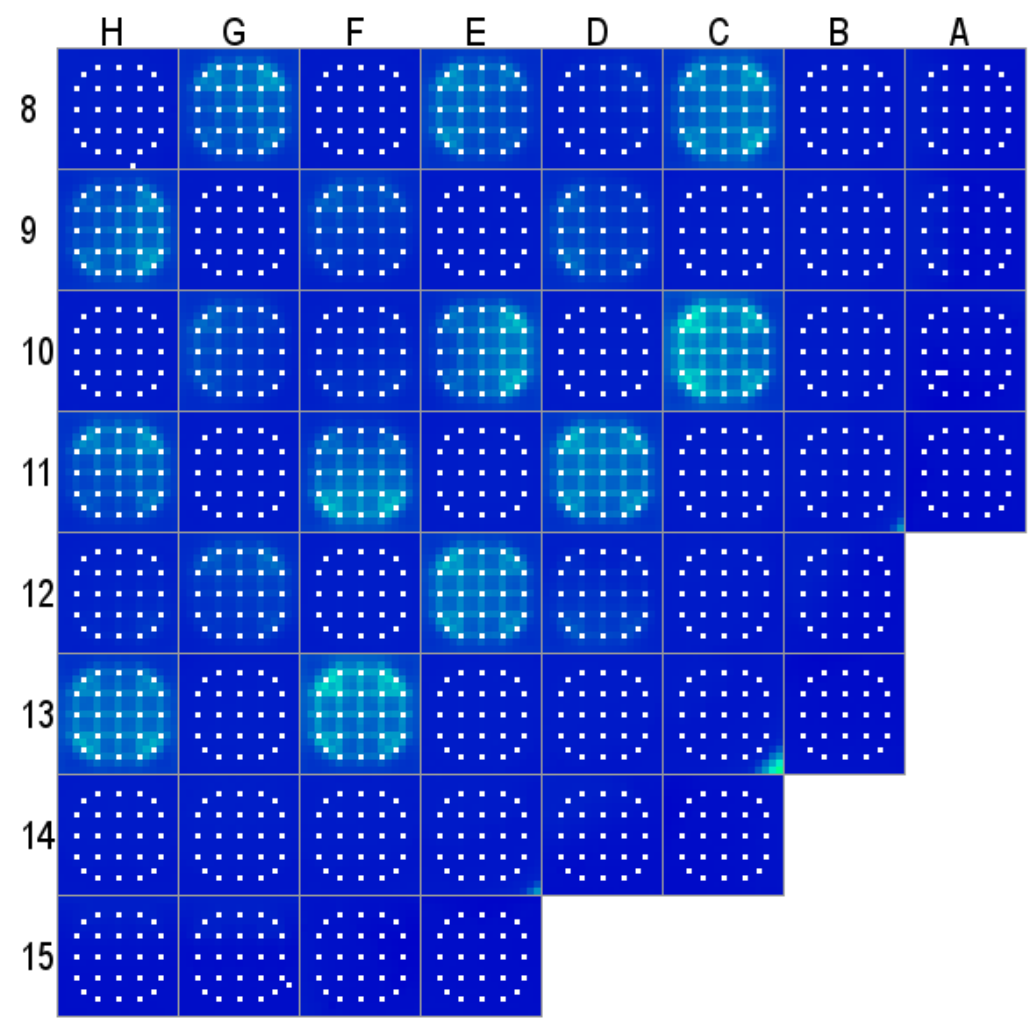

$7.65 \mathrm{e}-05-$
$6.86 \mathrm{e}-05$
$6.08 \mathrm{e}-05$
$5.29 \mathrm{e}-05$
$4.5 \mathrm{e}-05$
$3.71 \mathrm{e}-05$
$2.92 \mathrm{e}-05$
$2.13 \mathrm{e}-05$
$1.34 \mathrm{e}-05$
$5.48 \mathrm{e}-05$

Fig. A.3.40. Cycle 3 minimum gap thickness (m) - 19.32 GWd/MT. 


\section{APPENDIX B - UPDATED BISON INPUT TEMPLATE}

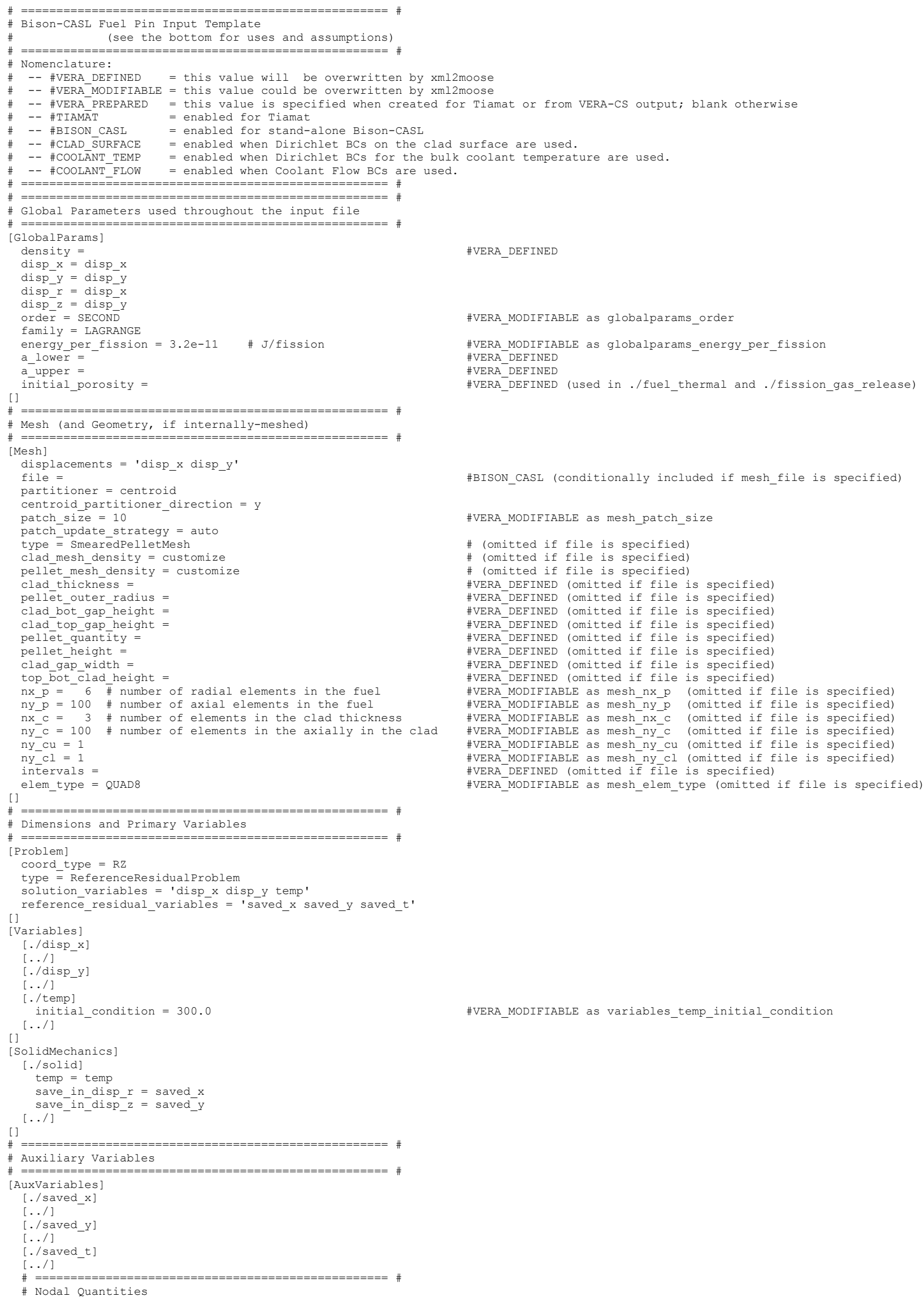

\#VERA_MODIFIABLE as globalparams_order

\#VERA_MODIFIABLE as globalparams_energy_per_fission \#VERA_DEFINED

\#VERA_DEFINED (used in ./fuel_thermal and./fission_gas_release)

\#BISON_CASL (conditionally included if mesh_file is specified)

\#VERA_MODIFIABLE as mesh_patch_size

\# (omitted if file is specified)

\# (omitted if file is specified)

\# (omitted if file is specified)

\#VERA DEFINED (omitted if file is specified)

\#VERA DEFINED (omitted if file is specified)

\#VERA DEFINED (omitted if file is specified)

\#VERA DEFINED (omitted if file is specified)

HVERA DEFTNED (omitted if file is specified)

\#VERA DEFINED (omitted if file is specified)

\#VERA_DEFINED (omitted if file is specified)

\#VERA DEFINED (omitted if file is specified)

\#VERA_MODIFIABLE as mesh_nx_p (omitted if file is specified) \#VERA_MODIFIABLE as mesh_ny_p (omitted if file is specified) \#VERA MODIFIABLE as mesh nx c (omitted if file is specified) \#VERA MODIFIABLE as mesh ny c (omitted if file is specified) \#VERA-MODTFTABIF as mesh nY HVRA_MODIFIABIE as mesh_n_cl (omitted if file is specified) (omitted if file is specified)

\#VERA_MODIFIABLE as mesh_elem_type (omitted if file is specified) 


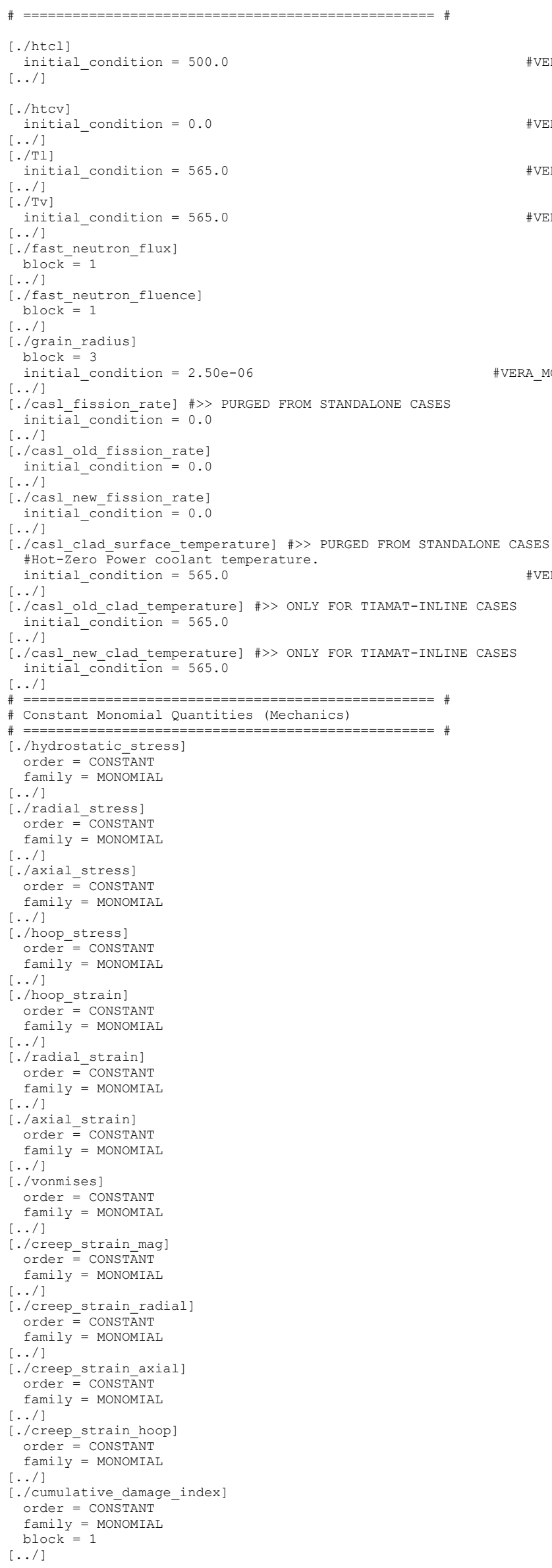




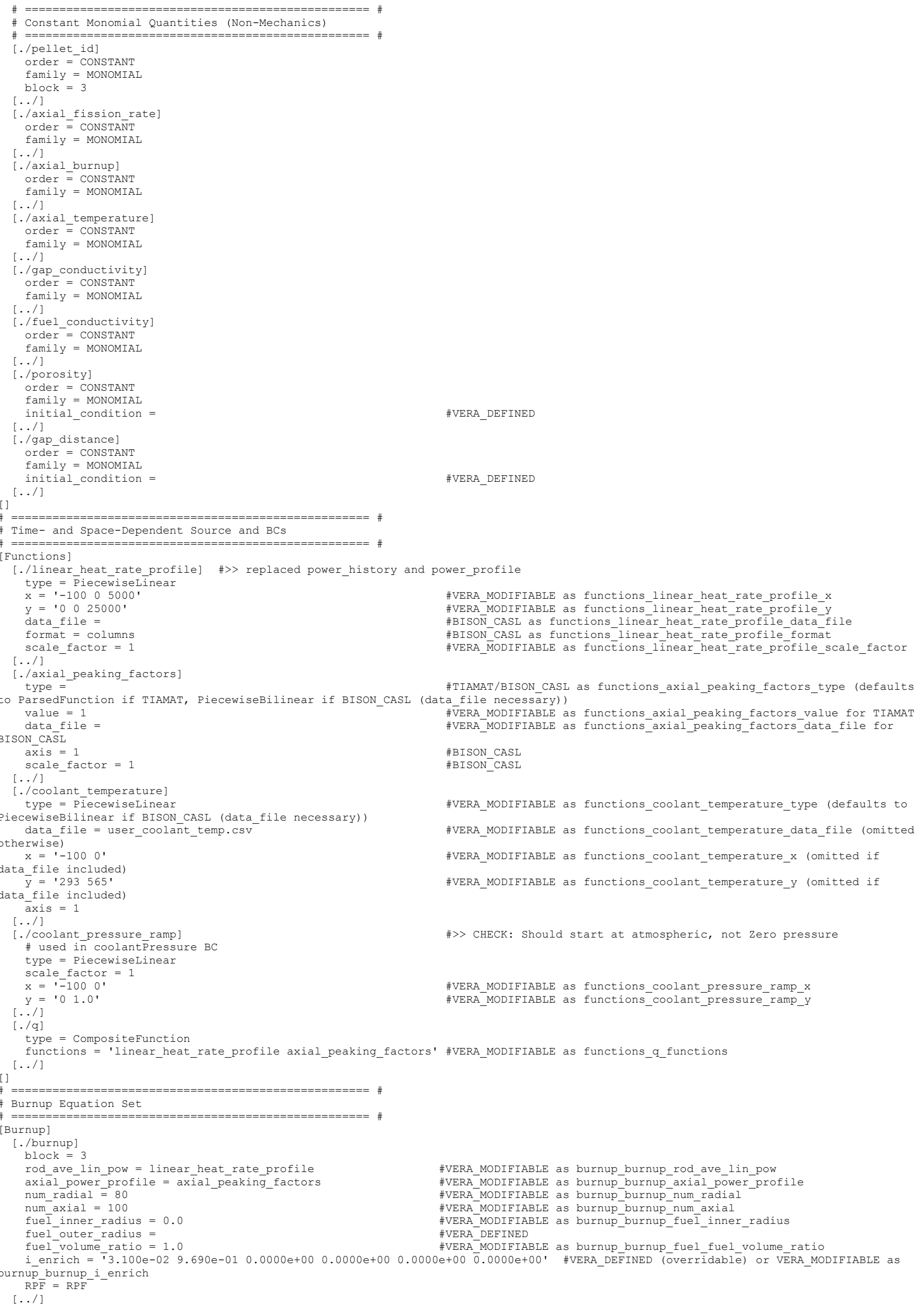




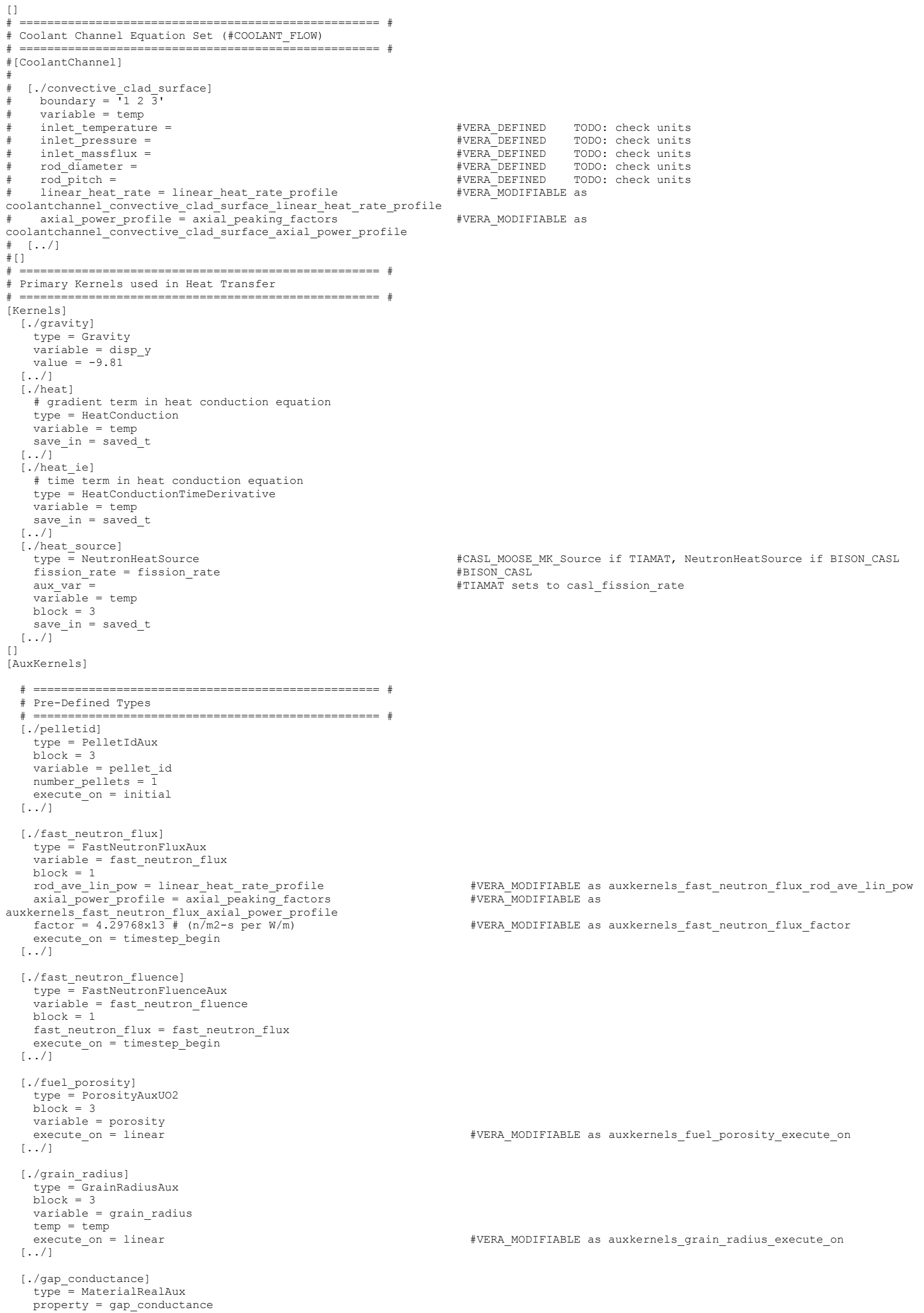




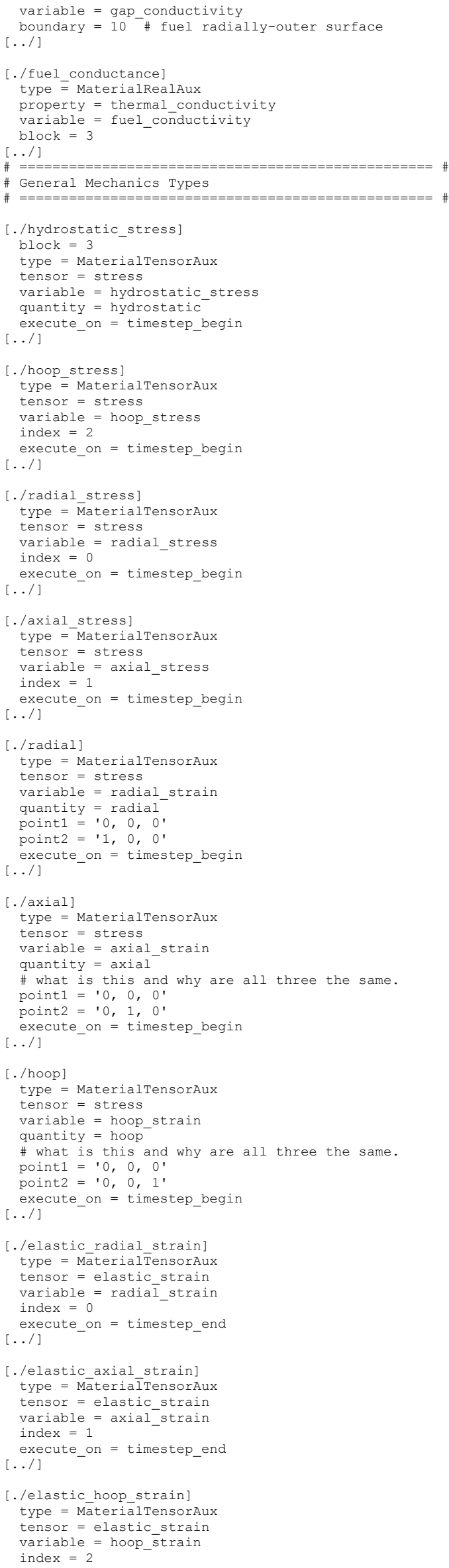

\#VERA_MODIFIABLE as auxkernels_hydrostatic_stress_execute_on

\#VERA_MODIFIABLE as auxkernels_hoop_stress_execute_on

\#VERA_MODIFIABLE as auxkernels_radial_stress_execute_on

\#VERA_MODIFIABLE as auxkernels_axial_stress_execute_on

\#VERA_MODIFIABLE as auxkernels_radial_execute_on

\#VERA_MODIFIABLE as auxkernels_axial_execute_on

\#VERA_MODIFIABLE as auxkernels_hoop_execute_on 


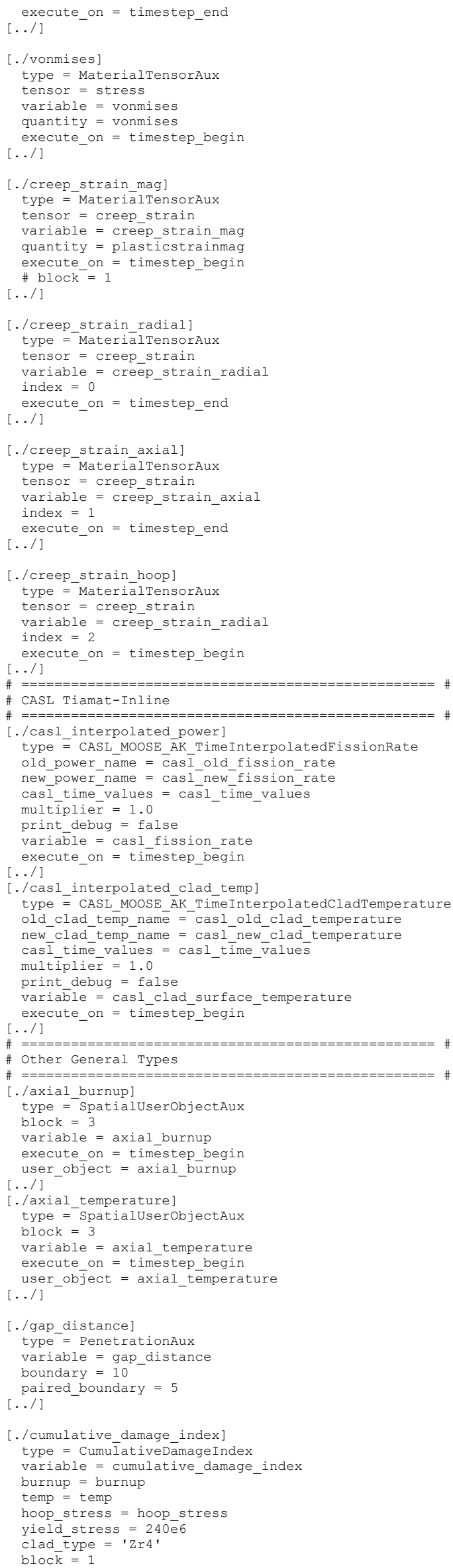




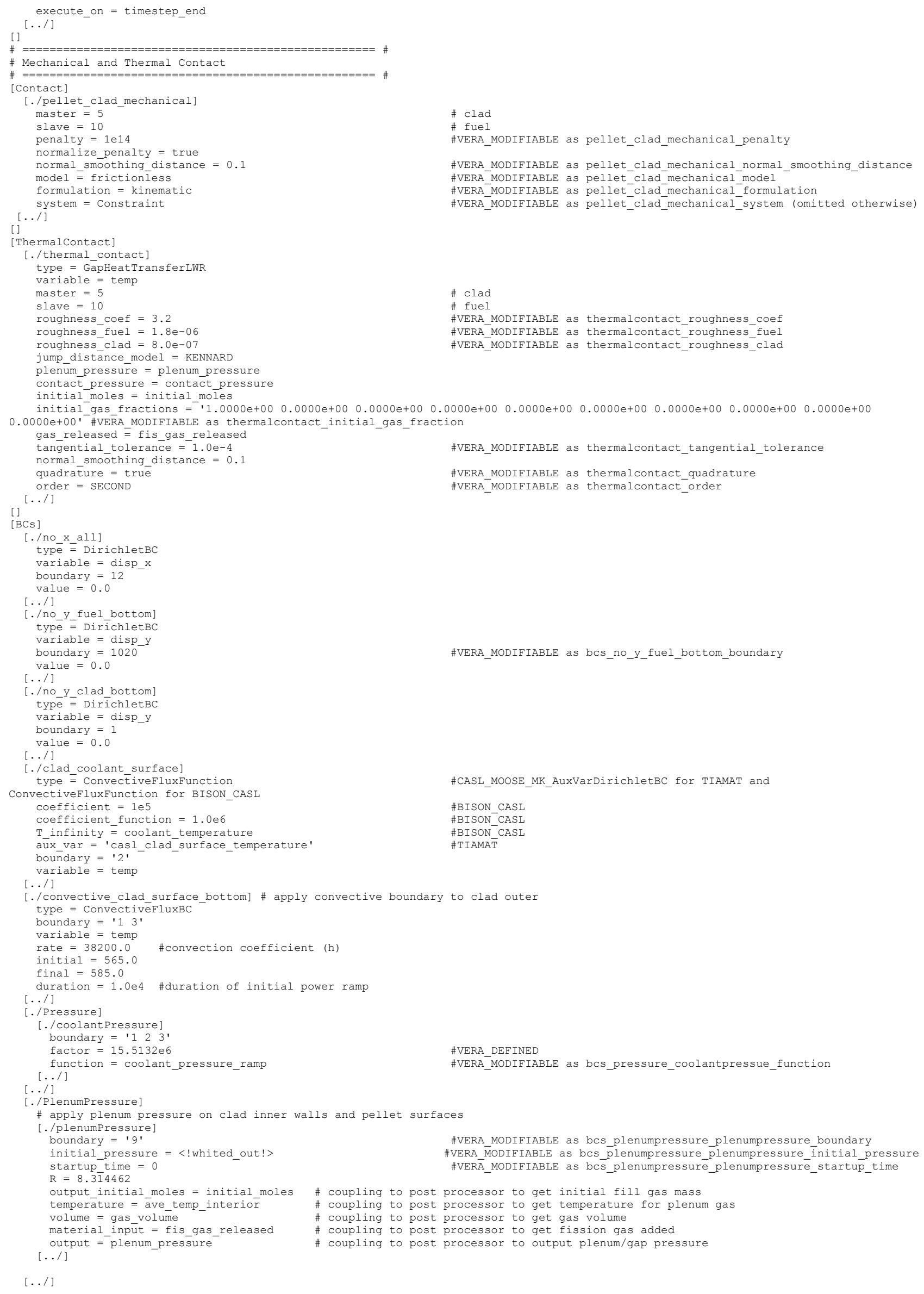




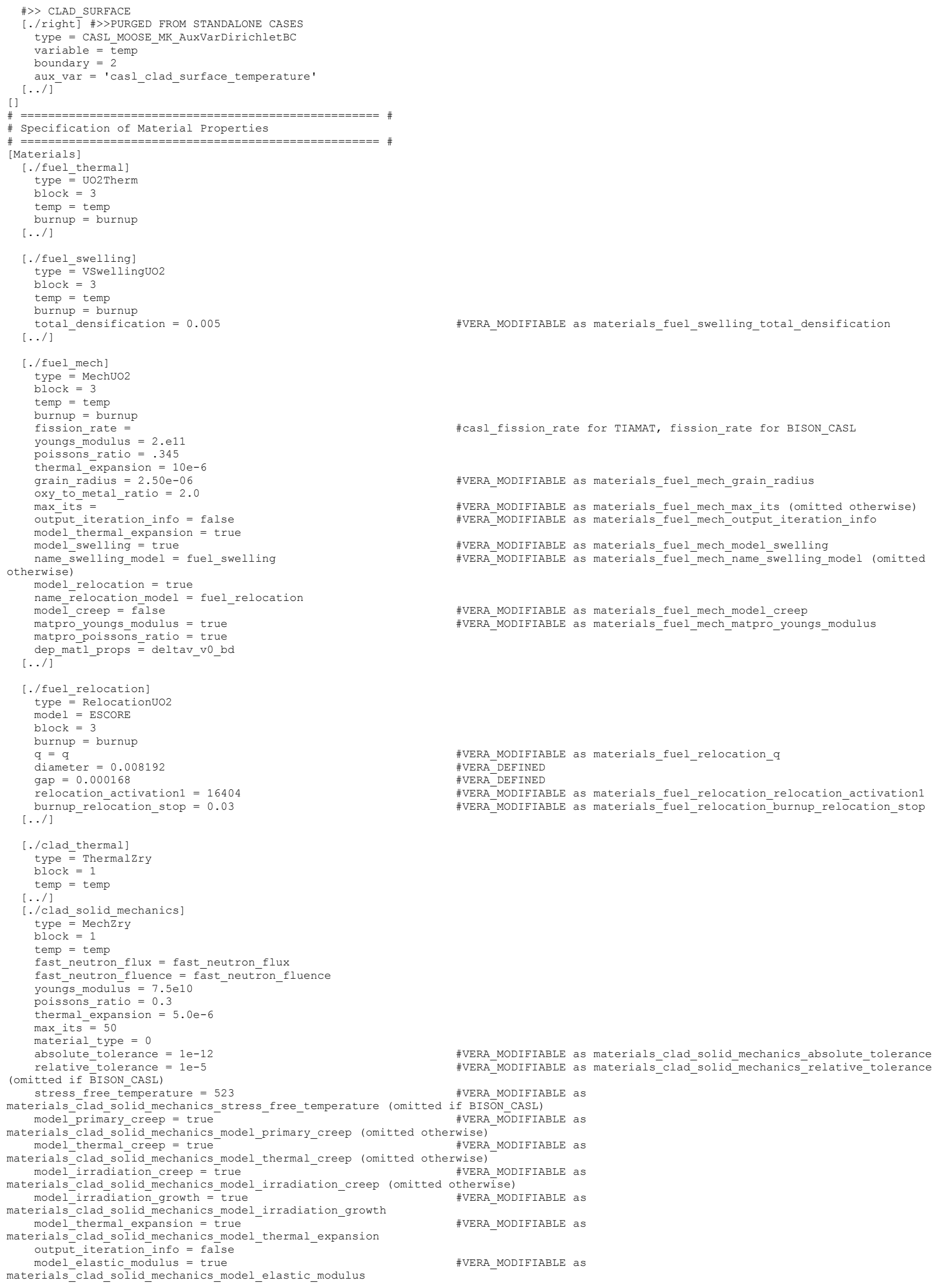

materials_clad_solid_mechanics model_irradiation_growth
model_thermal expansion $=$ true

\#VERA_MODIFIABLE as materials_clad_solid_mechanics_absolute_tolerance \#VERA MODIFIABLE as materials clad solid mechanics_relative_tolerance

\#VERA_MODIFIABLE as 


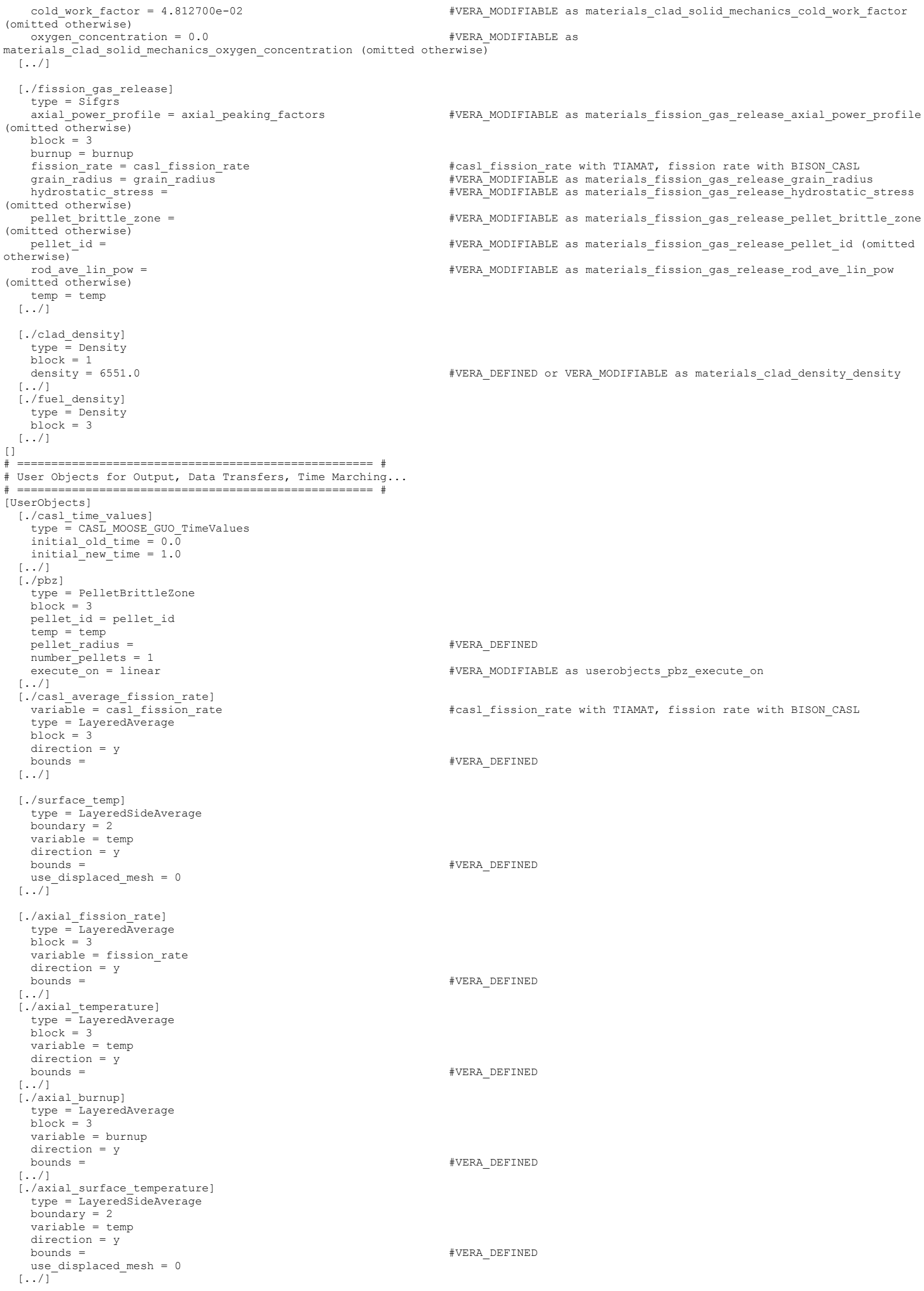




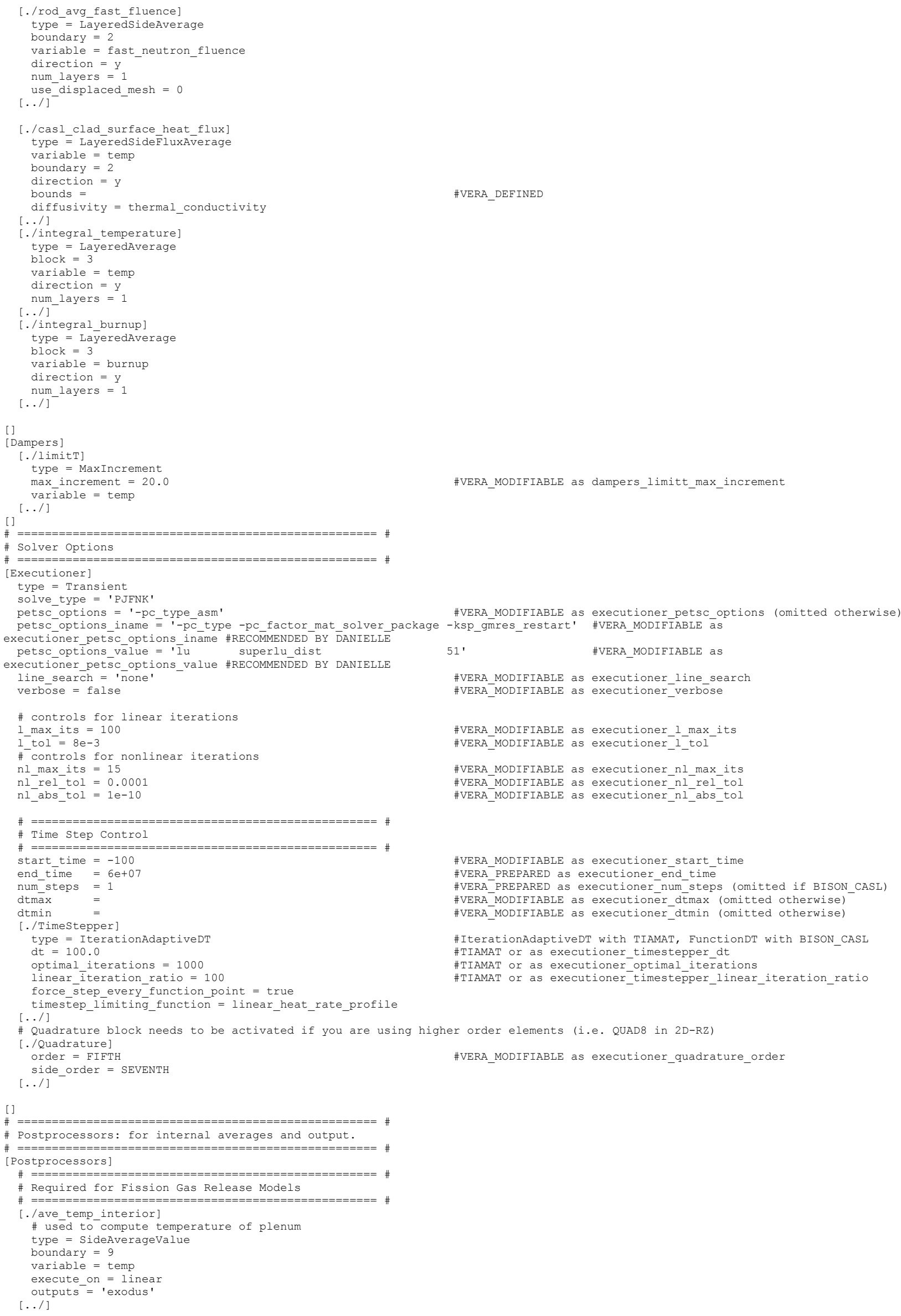


[./fis_gas_released]

type $=$ ElementIntegralFisGasReleasedSifgrs

variable = temp

block $=3$

outputs $=$ 'exodus'

$[\ldots /]$

[. /gas_volume]

type ${ }^{-}=$Internalvolume

boundary $=9$

execute_on $=$ linear

outputs $=$ 'exodus'

$[\ldots /]$

[./fis gas grain]

type $=$ ElementIntegralFisGasGrainSifgrs

variable $=$ temp

block $=3$

outputs $=$ exodus

$[\ldots /]$

[./fis_gas_boundary]

type $=$ ElementIntegralFisGasBoundarySifgrs

variable $=$ temp

block $=3$

outputs $=$ exodus

$[\ldots /]$

$============0$ \#

\# Output-Only: Fission Gas Release Data

\# $====================$

[ype $=$ ElementĀiverageValue

type $=\mathrm{El}$
block $=3$

outputs = 'exodus'

variable $=$ grain_radius

$[\ldots /]$

[./clad_inner_vol]

type $=$ InternalVolume

boundary $=7$

outputs $=$ 'exodus'

$[\ldots /$ ]

[./pellet volume]

type $=\overline{\text { InternalVolume }}$

boundary $=8$

outputs $=$ 'exodus'

$[\ldots /]$

[./avg_clad_temp]

type $=$ Si $\bar{d}$ eAverageValue

boundary $=7$

variable $=$ temp

outputs $=$ 'exodus'

$[\ldots /$ ]

[./fis_gas produced]

type $=$ ElementIntegralFisGasGeneratedSifgrs

variable = temp

block $=3$

outputs = 'exodus'

$[\ldots /]$

Output-Only: Gap-Conductance Related

[./flux from clad]

type $=$ SidéFluxIntegral

variable $=$ temp

boundary $=5$

diffusivity = thermal_conductivity

outputs = 'exodus'

$[\ldots /]$

[./flux_from_fuel]

type $=$ SidēFluxIntegral

variable $=$ temp

boundary $=10$

diffusivity = thermal conductivity

outputs $=$ 'exodus'

$[\ldots /]$

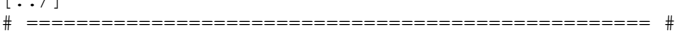

\# Output-Only: Solver Related

$\#=====+2==1$
$[. / \mathrm{dt}]$

type = TimestepSize

outputs $=$ 'exodus'

$[\ldots /]$

$[. /$ nonlinear_its $]$

type = NumN̄onlinearIterations

outputs $=$ 'exodus'

$[\ldots /]$

[./linear its] \# number of nonlinear iterations at each timestep

type $=\overline{\text { NumLinearIterations }}$

$[\ldots /]$

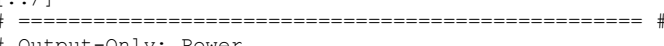

\# Output-Only: Power

[./rod_average_fuel_temp]

type $=$ ElementAverageValue

block $=3$

variable $=$ temp

$[\ldots /]$

[./rod_total_power]

type $=$ Element Integral Power

variable $=$ temp 


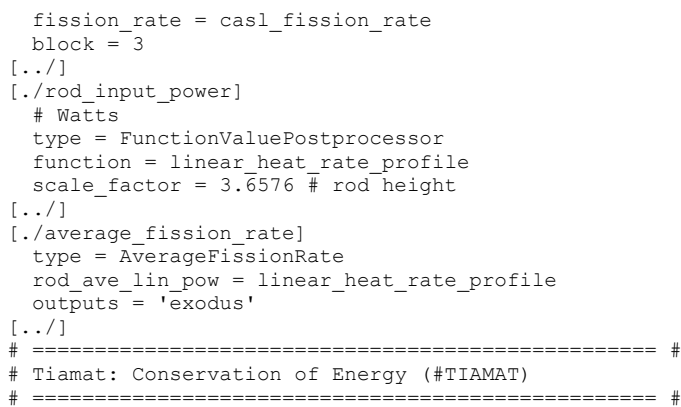

\#VERA_DEFINED

\#Casl_fission_rate with TIAMAT, fission_rate with BISON_CASL 


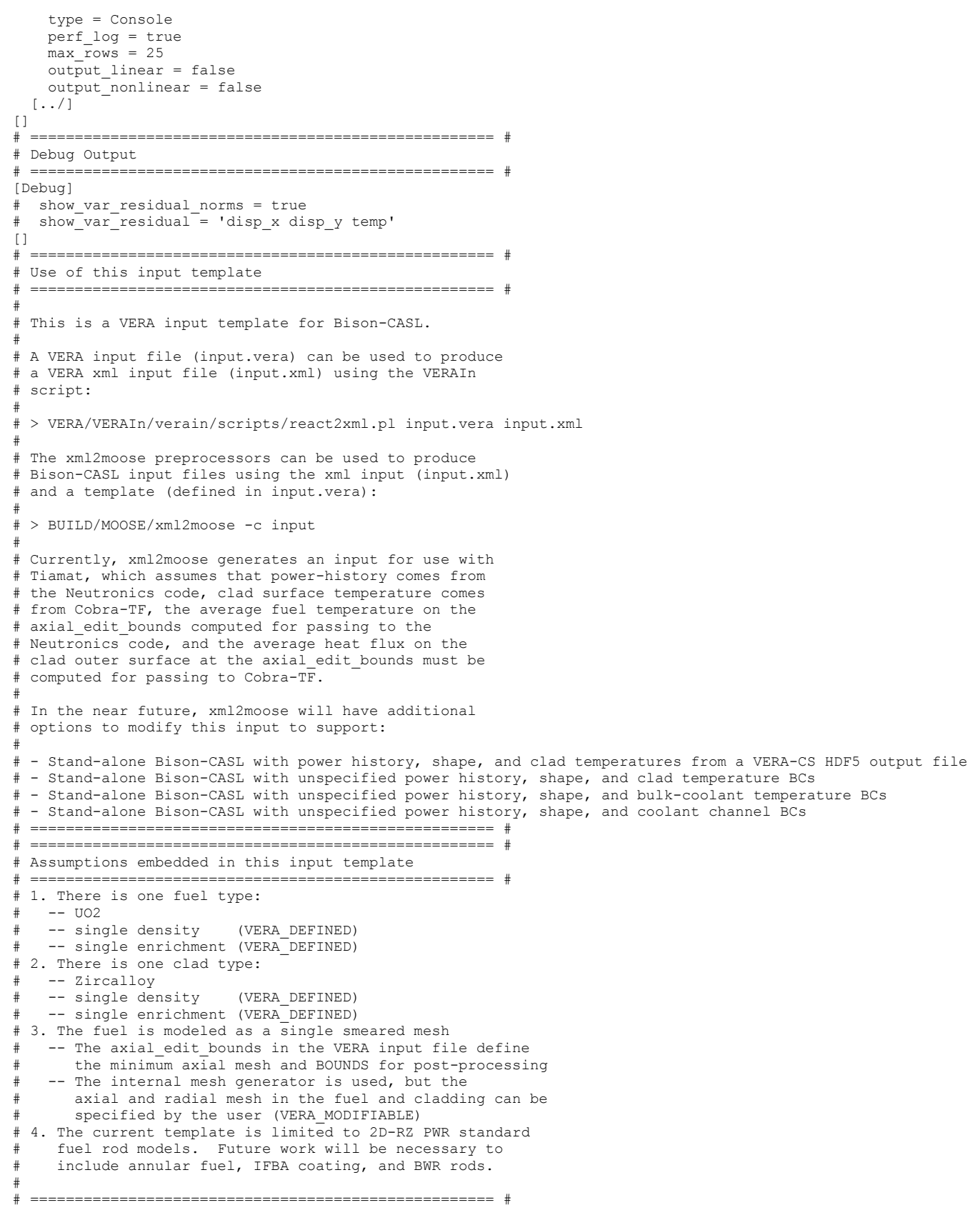

